\title{
Genome-wide association study of
}

\section{Pseudomonas aeruginosa adaptations}

\author{
to the cystic fibrosis lung \\ by
}

\section{Katherine Noah}

A thesis submitted to the Faculty of Graduate and Postdoctoral Affairs in partial fulfillment of the requirements for the degree of

\author{
Master of Science
}

in

Biology with Specialization in Data Science

\author{
Carleton University \\ Ottawa, Ontario
}

(C)2019

Katherine Noah 


\section{Abstract}

Pseudomonas aeruginosa is an opportunistic pathogen that chronically infects the lungs of patients with cystic fibrosis. We know that these bacteria evolve within the lung environment, and have an idea of some of the changes that occur. I performed a genome-wide association study in P. aeruginosa using a variety of analysis methods and several datasets of gene and SNP presence/absence. I determined that the machine learning algorithms random forest and support vector machines performed well on gene presence/absence and core SNP datasets, respectively, when compared with current methods (PLINK and treeWAS). Genes and SNPs already associated with adaptation to the CF lung environment, such as $m u c A$, gyrA, and mex genes, were found with these methods. Some hypothetical and probable proteins were also recovered, and are good candidates for future research. 


\section{ACKNOWLEDGEMENTS}

First and foremost, I want to thank Dr. Alex Wong for his endless guidance and support. I am thankful to him for keeping me on track with this project, but also allowing me the freedom to look into new methods. I am so grateful that he gave me a chance when my undergraduate grades were mediocre, at best. I have learned so much in the past two years with Alex as my mentor and advisor, and appreciate all the opportunities that have come from being a part of his lab. He has helped me grow as a scientist and as a person, and is so supportive of all of his students in and out of the lab.

Thank you to my committee members, Drs. Thien-Fah Mah and Azam Tayabali, for their insightful contributions and comments at progress meetings. I also want to thank Dr. Mah for looking into the hypothetical proteins in her lab.

I want to thank Calvin Jary and Dr. Jim Green, in Systems and Computer Engineering, for helping with the machine learning steps. I also want to thank Ryan Taylor for his fantastic help with bash scripts and cluster memory, and Wade Hong for being ever so patient with emails about the computing cluster and new programs to install.

To my lab mates, past and present, for their support and friendship. Although I was not in the wet lab, they were always great listeners when programs were crashing and when exciting results came in.

Thank you to JS and Kevin for believing in me and my abilities, and encouraging me to find new adventures.

Finally, to my parents and Tom, for your support over the last two years. Thank you for your patience and allowing to me drone on about code failures, computer crashes, and abstract results. I could not have pursued this project without your encouragement and guidance. I am so grateful to have you in my corner. 


\section{CONTENTS}

\begin{tabular}{ll}
\hline Abstract & i
\end{tabular}

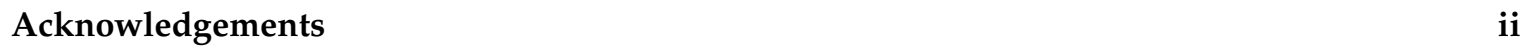

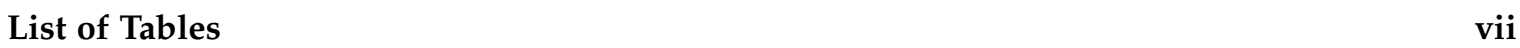

List of Figures $\quad x$

\begin{tabular}{lll}
\hline 1 & Introduction & 1
\end{tabular}

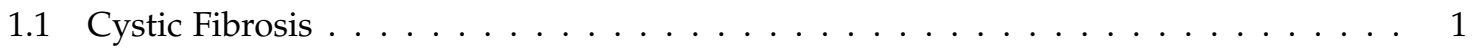

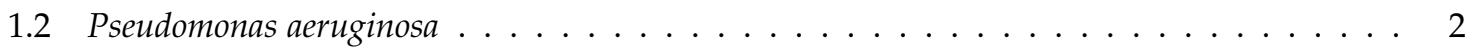

$1.2 .1 \quad$ Genome structure of P. aeruginosa $\ldots \ldots \ldots \ldots \ldots$

1.3 Genome-wide association studies . . . . . . . . . . . . . . . . . . . . . . . 7

1.3 .1 Bacterial GWAS $\ldots \ldots \ldots \ldots \ldots \ldots \ldots$

$1.4 \quad$ Machine Learning . . . . . . . . . . . . . . . . . . . . . . . . . . . . . . 14

1.4 .1 Random Forest . . . . . . . . . . . . . . . . . . . . . . . . . . . . . . . . 14

1.4 .2 Support Vector Machine $\ldots \ldots \ldots \ldots \ldots \ldots$

$1.4 .3 \quad$ Machine Learning and GWAS $\ldots \ldots \ldots \ldots \ldots$. . . . . . . . . . 15

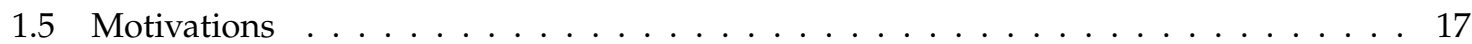

$\begin{array}{lll}2 & \text { Methods } & 17\end{array}$

2.1 Data collection and dataset curation . . . . . . . . . . . . . . . . . . 17

2.2 Phylogeny $\ldots \ldots \ldots \ldots \ldots \ldots$

2.3 fastStructure . . . . . . . . . . . . . . . . . . . . . . . . . . . . . . . 19

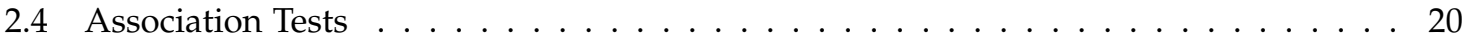

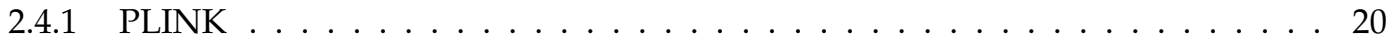

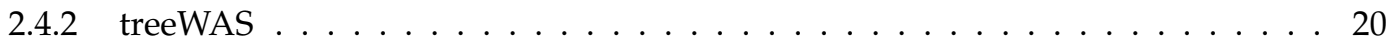

2.4 .3 Machine Learning Methods . . . . . . . . . . . . . . . . . . . . . . . . 21

2.4 .4 Other Statistical Tests $\ldots \ldots \ldots \ldots \ldots$. . . . . . . . . . . . . . . . . 21 
\begin{tabular}{lll}
\hline 3 & Dataset results & 21
\end{tabular}

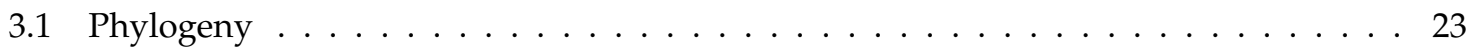

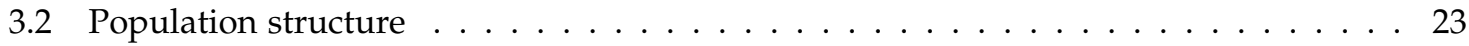

\begin{tabular}{|lll}
4 & Gene presence/absence dataset results and discussion & 25
\end{tabular}

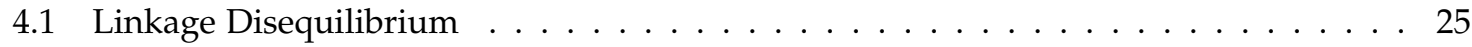

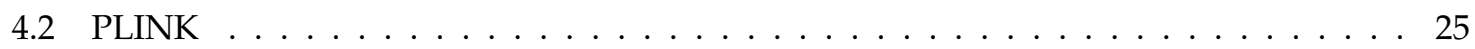

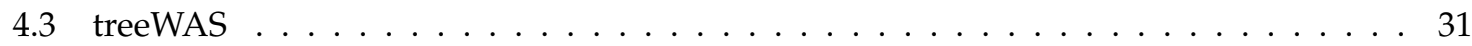

4.4 Random Forest $\ldots \ldots \ldots \ldots$. . . . . . . . . . . . . . . . . . . . . 33

4.5 Support vector machines $\ldots \ldots \ldots \ldots \ldots \ldots \ldots \ldots \ldots$

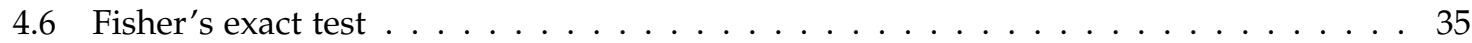

4.7 Principal component analysis $\ldots \ldots \ldots \ldots \ldots \ldots$

4.8 Conclusion of gene presence $/$ absence dataset $\ldots \ldots \ldots \ldots$. . . . . . . . . 37

5 Mutation dataset results and discussion $\quad 41$

5.1 Linkage Disequilibrium $\ldots \ldots \ldots \ldots \ldots \ldots \ldots \ldots \ldots \ldots$

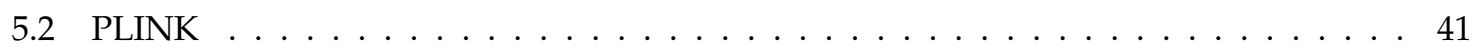

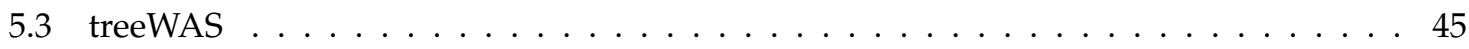

5.4 Random forest . . . . . . . . . . . . . . . . . . . . . . . . . . 45

5.5 Support vector machine $\ldots \ldots \ldots \ldots \ldots$. . . . . . . . . . . . 47

5.6 Fisher's exact test $\ldots \ldots \ldots \ldots \ldots \ldots \ldots \ldots$

5.7 Principal component analysis $\ldots \ldots \ldots \ldots \ldots \ldots$

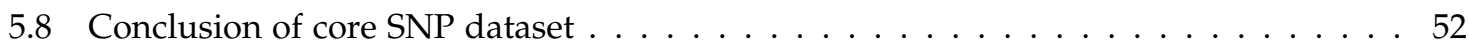

6 Gene and mutation dataset results and discussion 55

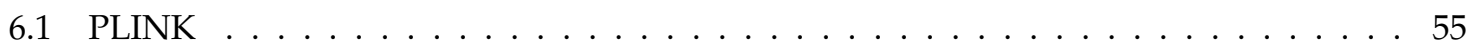

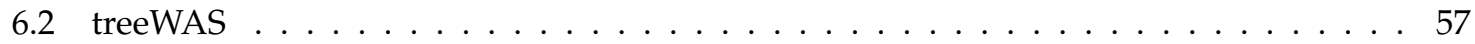

6.3 Random forest . . . . . . . . . . . . . . . . . . . . . . . . . . . . . . . . . . . . 59

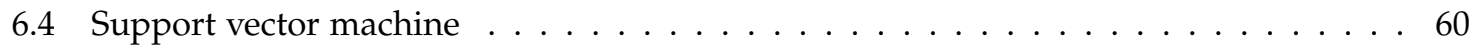

6.5 Conclusion . . . . . . . . . . . . . . . . . . . . . . . . 61 
\begin{tabular}{|lll}
7 & Select gene and mutation dataset results and discussion & 63
\end{tabular}

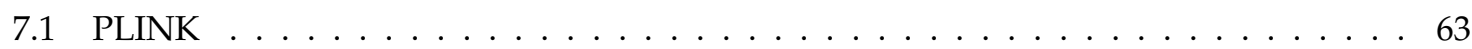

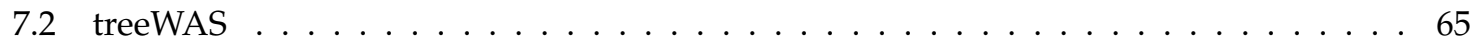

\begin{tabular}{lll}
\hline 8 & Randomizations & 68
\end{tabular}

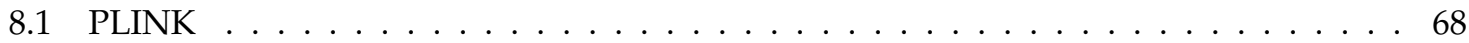

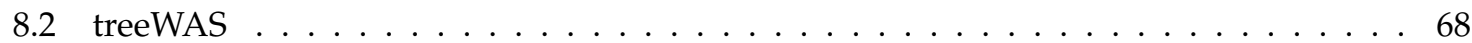

8.3 Random Forest and Support Vector Machine $\ldots \ldots \ldots$. . . . . . . . . . . . 70

8.4 Conclusion . . . . . . . . . . . . . . . . . . . . . . . . . . 70

\begin{tabular}{lll}
\hline & Conclusion & 70
\end{tabular}

\begin{tabular}{ll}
\hline References & 76
\end{tabular}

\begin{tabular}{|ll|}
\hline A Background information & 93
\end{tabular}

A.1 Genes/proteins associated with CF-phenotype of PA . . . . . . . . . . . . . . . 93

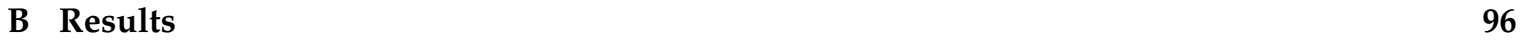

B.1 Dataset 1 PLINK results $\ldots \ldots \ldots \ldots$. . . . . . . . . . . . . . . . . 96

B.2 Dataset 1 treeWAS results $\ldots \ldots \ldots \ldots$. . . . . . . . . . . . . . 100

B.3 Dataset 1 random forest results . . . . . . . . . . . . . . . . . . . . . . . . . . . . 103

B.4 Dataset 1 support vector machine results $\ldots \ldots \ldots$. . . . . . . . . . . . . 106

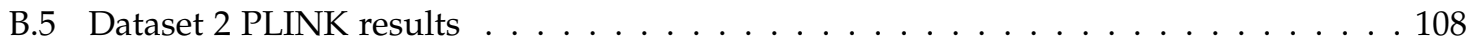

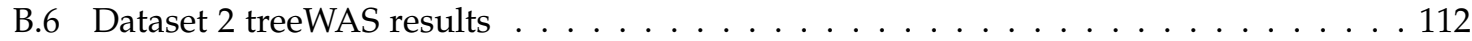

B.7 Dataset 2 random forest results . . . . . . . . . . . . . . . . . . . . . . . . . . . 115

B.8 Dataset 2 support vector machine results . . . . . . . . . . . . . . . . . . . . . . . . . . 119

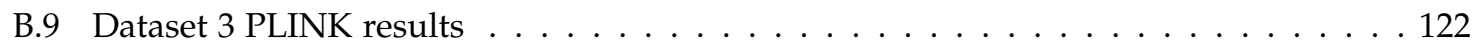

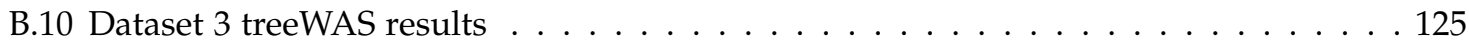

B.11 Dataset 3 random forest results . . . . . . . . . . . . . . . . . . . . . . . . . . . . . . . . 129

B.12 Dataset 3 support vector machine results . . . . . . . . . . . . . . . . . . 132

B.13 Dataset 4 PLINK results . . . . . . . . . . . . . . . . . . . . . . . . . . 135

B.14 Dataset 4 treeWAS results . . . . . . . . . . . . . . . . . . . . . . . . 139

B.15 Dataset 4 random forest results . . . . . . . . . . . . . . . . . . . . . . . . . 141 
CONTENTS

B.16 Dataset 4 support vector machine results $\ldots \ldots \ldots \ldots$. . . . . . . . . . 144

\begin{tabular}{lll}
\hline C Additional Test Results & 147
\end{tabular}

C.1 Results from random forest after condensing dataset 1 using 95\% similarity profiles . 147 


\section{LIST OF TABLES}

$1 \quad$ Published bacterial GWAS, by year. Number of variants is number of genes, SNPs or k-mers analyzed. . . . . . . . . . . . . . . . . . . . 12

2 Reference strains used for annotations in various studies. Known orthologs with PAO1 were used to identify genes in the 1597 genomes. $\ldots \ldots \ldots \ldots$. . . . . . 18

3 Description of datasets analyzed and their size. . . . . . . . . . . . . . . . . 19

$4 \quad$ Strains used for ordering of accessory genes. Only reference genomes with known orthologs to PAO1 that had 100 or more unaccounted for accessory genes were used. Ortholog information was retrieved from the online Pseudomonas database [1] . . . . 28

$5 \quad$ Top genes from from PLINK stratified association analysis $\ldots \ldots \ldots$. . . . . . . . . . . 29

$6 \quad$ Top genes found in treeWAS combined association analysis. . . . . . . . . . . . . 31

$7 \quad$ Top genes that contribute to the random forest model. . . . . . . . . . . . . 33

$8 \quad$ Top genes found in support vector machine model. . . . . . . . . . . . . . . . . 35

$9 \quad$ Top SNPs and indels from from PLINK stratified association analysis $\ldots \ldots \ldots$. . . . 44

$10 \quad$ Top SNPs and indels found in treeWAS combined association analysis. . . . . . . . . 45

11 Top SNPs and indels that contribute to the random forest model. . . . . . . . . . . . 47

12 Top SNPs and indels found in support vector machine model. . . . . . . . . . . . . . 47

13 Top genes and SNPs from from PLINK stratified association analysis. . . . . . . . . 55

14 Top genes and SNPs found in treeWAS simultaneous association analysis. . . . . . . 59

15 Top genes and SNPs that contribute to the random forest model. . . . . . . . . . . . . 59

16 Top genes and SNPs found in support vector machine model . . . . . . . . . . . . 60

17 Genes and proteins important for the CF-phenotype as identified in several review

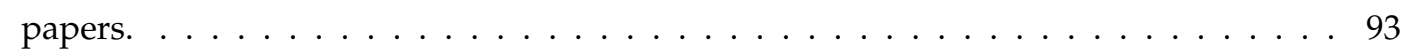

18 Genes identified with PLINK from dataset 1, gene presence/absence. Genes with $p$ values less than or equal to $2.54 \times 10^{-10}$ are reported. . . . . . . . . . . . . . 96

19 Genes identified with treeWAS from dataset 1 , gene presence/absence. . . . . . . . 100

20 Genes identified with RF from dataset 1, gene presence/absence. Genes with importances greater than or equal to 0.002 are reported. . . . . . . . . . . . . . . 103

21 Genes identified with SVM from dataset 1, gene presence/absence. Top 100 genes with absolute hyperplane coefficients greater than 0.03 are reported. . . . . . . . . . 106 
22 SNPs identified with PLINK from dataset 2, SNPs within core genes. Top 100 SNPs with $p$ values less than $1 \times 10^{-8}$ are reported. . . . . . . . . . . . . . . . . 109

23 SNPs identified with treeWAS from dataset 2, SNPs within core genes. SNPs with absolute simultaneous association scores greater than or equal to 26 are reported. . . 113

24 SNPs identified with RF from dataset 2, SNPs within core genes. SNPs with importances greater than or equal to 0.002 are reported. . . . . . . . . . . . . . . . . . 115

25 SNPs identified with SVM from dataset 2, SNPs within core genes. Top 100 SNPs

with absolute hyperplane coefficients greater than 0.03 are reported. . . . . . . . . . 119

26 Genes and SNPs identified with PLINK from dataset 3, combined gene presence/absence and SNPs within core genes. Top 100 variants with $p$ values less than $1 \times 10^{-8}$ are reported. 2640 genes had $p$ values less than $1 \times 10^{-8}$. . . . . . . . . . . . . 122

27 Genes and SNPs identified with treeWAS from dataset 3, combined gene presence/absence and SNPs within core genes. Variants with absolute simultaneous association scores greater than or equal to 26 are reported. . . . . . . . . . . . 125

28 Genes and SNPs identified with RF from dataset 3, combined gene presence/absence and SNPs within core genes. Variants with importances greater than or equal to 0.002 are reported. . . . . . . . . . . . . . . . . . . . . . . . 129

29 Genes and SNPs identified with SVM from dataset 3, combined gene presence/absence and SNPs within core genes. Top 100 variants with absolute hyperplane coefficients greater than 0.015 are reported. . . . . . . . . . . . . . . . . . . 132

30 Genes and SNPs identified with PLINK from dataset 4, reduced dataset using features identified from random forest in datasets 1 and 2. Variants with $p$ values less than

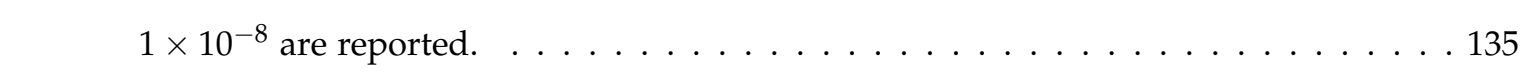

31 Genes and SNPs identified with treeWAS from dataset 4, reduced dataset using features identified from random forest in datasets 1 and 2. Variants are ranked based on absolute simultaneous association score. . . . . . . . . . . . . . . . . . . 139

32 Genes and SNPs identified with RF from dataset 4, reduced dataset using features identified from random forest in datasets 1 and 2. Variants with importances greater than or equal to 0.002 are reported. . . . . . . . . . . . . . . . . . 141 
LIST OF TABLES

33 Genes and SNPs identified with SVM from dataset 4, reduced dataset using features identified from random forest in datasets 1 and 2. Top 100 variants with absolute hyperplane coefficients greater than 0.03 are reported. . . . . . . . . . . . . . . 144

34 Importance of genes in random forest models before and after condensing the dataset using a $95 \%$ similarity threshold. If genes shared $95 \%$ of the same presence/absence profile with another gene(s), the genes were condensed into a single variable. The majority of genes condensed were either core genes or genes found in PA during a longitudinal study of one patient. . . . . . . . . . . . . . . . . . 147 


\section{List OF Figures}

$1 \quad$ Scatter plot of bacterial GWAS, by year published. Red datapoint is this study. . . . . 11

2 Example of decision tree, where the dataset is split on gene presence (1) or absence (0) to make homogenous groups of antimicrobial resistant (AMR) and non-resistant (no AMR) strains. . . . . . . . . . . . . . . . . . . . . . . . . . . 16

$3 \quad$ Example of support vector machine model with three variables: $x, y$ and $z$. Strains with phenotype blue and orange cannot be split by $x$ and $y$ (Panel 1), however they can be clearly split on axis $z$ (Panel 2). . . . . . . . . . . . . . . . . . . . . . . . . 16

$4 \quad$ Number of PAO1 genes present in proportion of strains in the dataset. A 95\% threshold was used for the core genome - if a gene was in 95\% or more of strains, it was labelled as a core gene. 4997 genes were part of the core genome. . . . . . . . . . 22

5 Phylogeny of 1576 P. aeruginosa genomes. Red squares outside the tree denote CF strains. Studies contributing $>100$ strains are highlighted (red and blue), as are studies with $>40$ strains found in a single clade (orange, pink and green). Branch colours show bootstrapping confidence, green is 100 , red is 1. . . . . . . . . . . . . . . 24

6 Proportion of cluster association for all strains. The $x$-axis is the strain, ordered by population. The y-axis is the proportion of belonging to a cluster. . . . . . . . . . . . 26

$7 \quad$ Linkage disequlibrium pattern in P. aeruginosa genes, $r^{2}$ as calculated in PLINK. The X-axis represents the location along the pan-genome. The first 3513 locations are \begin{tabular}{|l|l|l|l|}
\hline ordered as in strains from Table & 4 & The last 6000 positions are ordered as found in \\
\hline
\end{tabular}

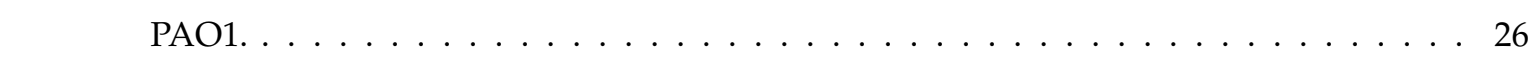

$8 \quad$ QQ plot of calculated $\log p$ values in PLINK of the gene dataset against expected $p$ values. The red line is when the observed $p$ values equal the expected $p$ values $(\mathrm{x}=\mathrm{y}) . \quad 27$

9 Manhattan plot of $p$ values in the PLINK analysis of the gene dataset. Points above the red line are significant, and coloured in green. The red line is the genome-wide significance line, at $\log \left(1 \times 10^{-8}\right)$. The blue line is the suggestive significance line, at

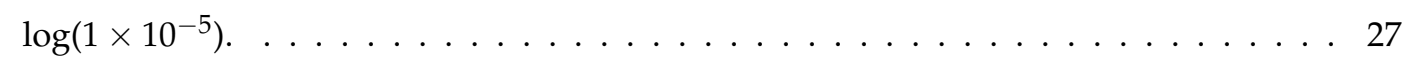

10 Manhattan plot of gene scores found from treeWAS association simultaneous analysis. 32

11 Hyperplane coefficient from linear support vector machines. Gene index was sorted based on lowest SVM coefficient to highest, to show the spread of values. . . . . . . . 36 
12 Manhattan plot of $p$ values from a Fisher's exact test for each gene found in any of the 1600 tested P. aeruginosa strains. The red line is the genome-wide significance line, at $\log \left(1 \times 10^{-8}\right)$. The blue line is the suggestive significance line, at $\log \left(1 \times 10^{-5}\right)$. . . 36

13 Boxplot of mean principal component values of genes for cystic fibrosis and non-cystic fibrosis strains. CF_flag of 1 is a cystic fibrosis strain, CF_flag of 0 is a non-cystic fibrosis strain. A) Principal component 2 mean values, B) principal component 3 mean values. . . . . . . . . . . . . . . . . . . . . . . . . . 38

14 Plot of principal component 2 values against principal component 1 for genes of cystic fibrosis and non-cystic fibrosis P. aeruginosa strains. . . . . . . . . . . . . . . 39

15 Venn diagram of results from PLINK, treeWAS, random forest and support vector machine. Genes identified in at least 3 of the 4 methods are reported. . . . . . . . . . 40

16 Heatmap of genes found in the gene presence/absence dataset with various methods. Darker blue is a higher ranking (lower $p$ value or higher association score/ importance/ hyperplane coefficient) gene. Yellow is a lower ranking variant. White is not identified as contributing to the phenotype. . . . . . . . . . . . . . . . . . 40

17 Linkage disequlibrium pattern in P. aeruginosa core genome mutations, as calculated in PLINK. The $x$-axis represents the location along the PAO1 genome. . . . . . . . . . 42

18 Linkage disequlibrium $\left(r^{2}\right)$ decay in P. aeruginosa . . . . . . . . . . . . . . 42

19 QQ plot of calculated $\log p$ values in PLINK of the mutation dataset against expected $p$ values. The red line represents when observed $p$ values equal the expected $p$ values

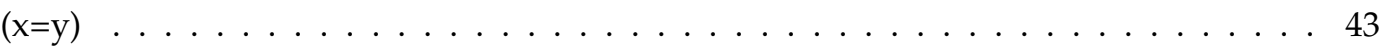

20 Manhattan plot of $p$ values in the PLINK analysis of the SNP dataset. Points above the red line are significant, and coloured in green. The red line depicts the genome-wide significance line, at $\log \left(1 \times 10^{-8}\right)$. The blue line represents the suggestive significance

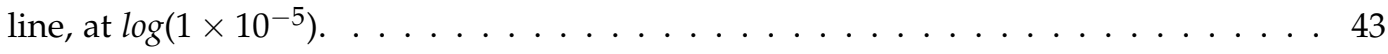

21 Manhattan plot of SNP scores found from treeWAS association simultaneous analysis. 46

22 Manhattan plot of $p$ values from a Fisher's exact test for each SNP found in a core gene. The red line is the genome-wide significance line, at $\log \left(1 \times 10^{-8}\right)$. The blue line is the suggestive significance $\operatorname{line}$, at $\log \left(1 \times 10^{-5}\right) . \ldots \ldots \ldots \ldots$. . . . . . 50 
23 Boxplot of mean principal component values of mutations for cystic fibrosis and non-cystic fibrosis strains. CF_flag of 1 is a cystic fibrosis strain, $\mathrm{CF}_{-}$flag of 0 is a non-cystic fibrosis strain. A) Principal component 2 mean values, B) principal component 3 mean values. . . . . . . . . . . . . . . . . . . . . . . . 50

24 Plot of principal component 2 values against principal component 1 for mutations of cystic fibrosis and non-cystic fibrosis P. aeruginosa strains. . . . . . . . . . . . . . 51

25 Venn diagram of results from PLINK, treeWAS, random forest and support vector machine. SNPs identified in at least 3 of the 4 methods are reported, if there were

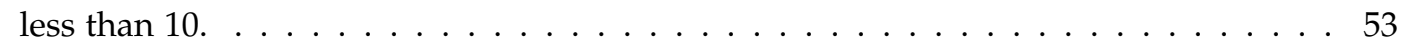

26 Heatmap of SNPs found in the core SNPs dataset with various methods. Darker blue is a higher ranking (lower $p$ value or higher association score/ importance/ hyperplane coefficient) variant. Yellow is a lower ranking variant. White is not identified as contributing to the phenotype. . . . . . . . . . . . . . . . . . 54

27 QQ plot of calculated $\log p$ values in PLINK of the gene and SNP datasets against expected $p$ values. The red line is when the observed $p$ values equal the expected $p$ values $(\mathrm{x}=\mathrm{y})$

28 Manhattan plot of $p$ values in the PLINK analysis of the gene and SNP dataset. Points above the red line are significant, and coloured in green. The red line is the genome-wide significance line, at $\log \left(1 \times 10^{-8}\right)$. The blue line is the suggestive significance line, at $\log \left(1 \times 10^{-5}\right)$. . . . . . . . . . . . . . . . . . . 56

29 Manhattan plot of gene and SNP scores found from treeWAS association simultaneous

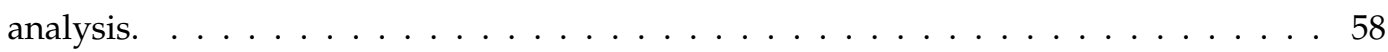

$30 \quad$ Venn diagram of results from PLINK, treeWAS, random forest and support vector machine. Variants identified in at least 3 of the 4 methods are reported. . . . . . . . . 62

31 Heatmap of variants found in the combined gene and SNP datasets with various methods. Darker blue is a higher ranking (lower $p$ value or higher association score/ importance/ hyperplane coefficient) variant. Yellow is a lower ranking variant. White is not identified as contributing to the phenotype. . . . . . . . . . . . . . . 62 
32 Heatmap of genes and SNPs found in the reduced dataset with various methods.

Dark blue is a higher ranking (lower $p$ value or higher association score/ importance/ hyperplane coefficient) variant. Yellow is a lower ranking variant. White is not identified as contributing to the phenotype. . . . . . . . . . . . . . . . . . . . 64

33 QQ plot of calculated $\log p$ values in PLINK of the reduced gene and SNP datasets against expected $p$ values. The red line is when the observed $p$ values equal the expected $p$ values $(\mathrm{x}=\mathrm{y})$

34 Manhattan plot of $p$ values in the PLINK analysis of the reduced gene and SNP dataset. Points above the red line are significant, and coloured in green. The red line is the genome-wide significance line, at $\log \left(1 \times 10^{-8}\right)$. The blue line is the suggestive significance line, at $\log \left(1 \times 10^{-5}\right) . \ldots \ldots \ldots \ldots \ldots 6$

35 Manhattan plot of reduced gene and SNP scores found from treeWAS association simultaneous analysis. . . . . . . . . . . . . . . . . . 67

36 Distribution of p-values from 1000 randomized PLINK model iterations. Blue, shaded line is the distribution of $\mathrm{p}$-values from the original run. . . . . . . . . . . . . . . . . 69

37 Distribution of $\mathrm{p}$-values from 1000 randomized treeWAS simultaneous association model iterations. Blue, shaded line is the distribution of p-values from the original run. 69

38 Accuracy of 1000 randomized random forest model iterations. Red line is the accuracy of the model with original data. . . . . . . . . . . . . . . . . . . . . . . . 71

39 Accuracy of 1000 randomized support vector machine model iterations. Red line is the accuracy of the model with original data. . . . . . . . . . . . . . . . . . . . 71

40 Variants in common between datasets for the various methods. A) PLINK, B) treeWAS, C) random forest, D) support vector machine. Datasets 1 and 2 (gene presence/absence and core SNPs) had no variants in common, as illustrated by blank spaces in the Venn diagrams. . . . . . . . . . . . . . . . . . . 73 


\section{INTRODUCTION}

\subsection{Cystic Fibrosis}

Cystic fibrosis (CF) is a genetic disease that primarily affects lung function in afflicted individuals. It is one of the most common fatal genetic diseases [2], with greater than 1 in 3600 children in Canada born with CF [3]. Respiratory problems, such as shortness of breath, persistent coughing, and lung infections, are the most common symptoms. Other symptoms of cystic fibrosis include problems with bowel movements and digestion of nutrients [4].

CF is usually diagnosed at birth with a sweat test. In 1948 clinicians discovered that CF involves an imbalance in electrolytes, not just a defect with mucus production, such that testing the sweat can detect these imbalances and lead to a CF diagnosis. A more thorough understanding of $\mathrm{CF}$ and novel treatment of associated lung infections has increased the median lifespan of CF patients. In 1970, about half of patients with CF did not live to their twenties, but patients born in 2019 are expected to live until their late-thirties to mid-forties [5, 6, 7]. There is currently no cure for cystic fibrosis, only treatment of the symptoms. Gene therapies have been investigated and there is promising research with new technologies, but nothing has made it to market [8, 9].

There are over a thousand mutations in the CFTR (cystic fibrosis transmembrane conductance regulator) gene that cause $\mathrm{CF}$, however most patients with $\mathrm{CF}$ have one of a select few mutations. The most common mutation is a deletion of phenylalanine at position 508, and most often affects those of European descent [4]. The CFTR protein regulates chlorine and sodium ion concentrations along the epithelium, and can affect the amount of liquid volume. Fluid may build in the lungs, if ion concentrations are not regulated properly, which is an ideal environment for a variety of pathogens which may cause lung infections in CF patients.

Chronic lung infections are a strong predictor of mortality in CF patients, where lung issues cause death in 80 to $95 \%$ of patients [10, 11]. Various bacteria infect the CF lung, and while some have little effect on the decline of lung function (e.g. Stenotrophomonas maltophilia, even when resistant to antibiotics), others have a marked effect, such as Burkholderia cepacia and Pseudomonas aeruginosa (PA) [12]. Staphylococcus aureus and Haemophilus influenzae are bacteria that usually infect CF lungs during childhood, but have not been shown to contribute to respiratory failure. They are replaced by Pseudomonas aeruginosa (PA) in later years, which is significantly more problematic [10]. 
Burkholderia cepacia is not as prevalent in CF patients, but can also cause severe lung infections and contribute to respiratory decline [13]. B. cepacia is especially a concern after lung transplants, while PA causes chronic infections - both contribute to the morbidity and mortality of CF patients.

\subsection{Pseudomonas aeruginosa}

PA is a ubiquitous bacterium that has been found to undergo genetic level changes after infecting the CF lung. In later stages of colonization, infections become chronic and impossible to clear [11, 12, 14, 15]. PA infections typically becomes a chronic during a patient's adolescence [6, 14]. Once a chronic infection takes hold, the goal of clinicians is no longer to clear the infection, but rather to manage it and try to keep lung function high. [12]. One theory for the difficulty in clearing bacterial infections is that the microbes get trapped in the lung fluid due to it being less fluid than that of healthy people. In addition, airways of CF patients have a reduced ability to clear invading bacteria [12]. Many antimicrobial treatments are used to treat these infections - preference is to use aerosol therapies as these can directly target the lung sputum while avoiding systemic adverse effects. However in more severe infections intravenous antibiotics are needed [12]. Intermittent infections can be effectively treated by antibiotics and this delays the onset of chronic infection. When reinfection occurs, $25 \%$ of the time PA will have the same genotype, suggesting that the initial infection was not completely eliminated [6]. If PA has a different genotype, it suggests new colonization from the environment, or that another undetected genotype within the patient has re-infected the host [6].

PA has been widely studied in the context of CF. There are longitudinal studies that will follow one or a few patients and their infections over time [16, 17, 18, 19], studies of epidemic strains [20, 21] and samples from CF clinics [11, 22, 23]. This has helped characterize the evolution and colonization of PA in a CF lung environment. Acquisition of PA infections in CF patients is usually from the environment, not from patient to patient transfer [7, 23].

There are significant trends in PA in the adaptation to their environment in chronic infections. Strains often become mucoid during the early stages of a chronic infection, but may lose this mucoidy in late infections [7, 17, 24]. They will form biofilms, develop resistance to antibiotics, adapt metabolic pathways, change in virulence, and have an increased mutation rate [6]. This evidence for convergent evolution among chronic PA strains emphasizes a need to understand 
the parameters driving evolution and the adaptations that arise to better control and treat these infections.

PA may become mucoid and form biofilms after chronic infection, making the infections harder to clear. Mucoidy is caused by an overproduction of alginate, which creates a glycocalyx around the bacterium [6]. muc genes regulate alignate production genes (alg, most importantly algD and algU). Loss of function mutations in muc genes can lead to the formation of mucoid mutants [24, 7]. Many of these mutations are found within the $m u c A$ gene. It is not uncommon for PA to revert back to a non-mucoid phenotype after chronic infection, either via a back-mutation or a secondary mutation in another gene [6, 7, 24]. This is due to a change in selective pressures in the environment and the fact that producing more alginate can be costly for the organism.

The lack of regulation of algU can lead to other adaptations, in addition to mucoidy. These changes include increases in heat shock, oxidative-stress and osmotic-stress responses, while inhibiting the type 3 secretion system and the Rhl quorum sensing signal [6, 7]. A higher production of alginate protects the bacteria from environmental stress and harsh lung conditions during a host inflammation response [6, 7].

PA forms subpopulations within a CF lung with different phenotypes. Part of this is due to a diverse respiratory environment, where, for example, the nasal passages have different nutrients compared to the lung alveoli [6]. Some subpopulations will become specialists in their niche, while others will remain generalists. Different populations may lose or gain functions to help the population of PA survive - some may be more mobile, some may be better suited to an anaerobic environment, while others may be more virulent.

Some subpopulations form small colony variants, made up of adherent bacterial cells, possibly due to the upregulation of pellicle and extracellular matrix genes (pel and $p s l$ ) [7. 24]. Small colony variants are able to persist in the lung environment longer and have greater resistance to antibiotics [25]. In addition, isolates with mutations in pel and psl genes are better suited to form biofilms, having developed an aggregate population already [7, 24].

Many mucoid strains of PA have reduced motility, since biofilms reduce the need for movement. Biofilms are communities of bacteria where there is an extracellular matrix of polysaccharides, proteins and DNA. Flagella are often repressed, but strains can re-gain their flagella with the reversion of mucoidy [7, 24]. The microbes in a biofilm are much more resistant to antibiotics than 
free-living microbes. It is more challenging for antibiotics to move through the extracellular matrix in a biofilm, thus they cannot reach their target of action. Biofilms also have novel methods to decrease their sensitivity to antibiotics. $n d v B$ is involved in the production of periplasmic glucans, which are thought to bind to antimicrobials and prevent them from binding to their target site [26]. Two-component regulatory systems involved in biofilm formation may also play a role in antibiotic resistance. $t c t D$ and $t c d E$, when present, reduce the expression of porin $\mathrm{OpdH}$, which in turn reduces the isolate's susceptibility to antibiotics [27, 28].

Biofilms are formed when there is an increase in alignate and the bacteria become more mucoid. Biofilm formation prompts a strong immune response from a host and often defines the beginning of a chronic PA infection in CF patients [6, 7]. Extracellular components can also be shared by the bacteria forming the biofilm, such as quorum sensing molecules [7], meaning biofilms can house heterogenous PA communities that work together to benefit the population. There can be differences in nutrient usage, energy expenditure, and antibiotic resistance profile in different locations within the biofilm [7].

PA is able to chronically infect the CF lung by adhering to the lung epithelium, losing its motility, and evading the immune system. These functions are believed to have related methods-ofaction: in the overproduction of alignate, the flagellum formation is suppressed to re-direct energy expenditure towards alginate production. This allows the bacterium to embed itself in the epithelial lung cells, and allow it to go unrecognized by the immune system [7, 24]. Other adaptations can also increase the success of PA colonization, such as the loss of pili (mutations or losses of pilB, pilQ, or rpoN) which aids in the adherence of the bacteria to the lung.

Acute and chronic PA infections are treated with a variety of antibiotics. The bacteria become resistant over time, especially in the case of chronic infections. Longitudinal studies have repeatedly observed the evolution of resistance by sampling isolates from a single patient over the course of several years [16, 17, 19]. There are a variety of mechanisms that confer antimicrobial resistance (AMR), and these mutations within CF PA isolates are related to the treatment an individual has received over the course of their infection. AMR-conferring mutations are generally de novo since guidelines for people living with CF has limited the transfer of infections between individuals [6].

The evolution of PA resistance to aminoglycosides, beta-lactams, ciprofloxacin, colistin, tobramycin, and others (including multi-drug resistance) has been observed and studied in previous 
years [7]. Mutations that confer resistance co-evolve in various lineages of PA.

Many of the mutations that confer resistance are found in efflux pumps (mex genes) or porins (omp or phoE genes). Changes to the functionality of these proteins can provide multi-drug resistance since the bacterium will have decreased uptake and increased expulsion of the drug. Beta-lactams, carbapenems and fluoroquinolones are some of the drug classes affected by these changes [29, 30]. Mutations in $g y r A, g y r B$, parC or parE, which encode for drug target enzymes DNA gyrase and topoisomerase IV, can cause resistance to fluoroquinolones [31]. Common resistance mutations include S83L, S83R and D87Y, which alter the binding site of quinolones in gyrase A or topoisomerase IV [32, 33, 34]. Similarly, resistance can be caused by deletions and SNPs in other proteins, such as transcription (e.g. S531L in $r p o B$ ) and translation factors (e.g. T12R in fmt or P413L in fusA), which confer resistance to specific antibiotics [34]. Overexpression of ampC results in resistance to beta-lactams [35, 36].

Many CF-related adaptations in PA are associated with metabolic pathways. Many chronic PA strains become unable to produce all required amino acids [24]. Changes to the PQS (Pseudomonas quinone signal), a quorum sensing molecule, are also common. Both mutations and fluctuations in nutrition can alter the PQS, which in turn causes changes to gene expression and regulation in the bacteria [24]. PA in chronic affections appear to prefer amino acids over glucose as a source of carbon [24]. Increased expression of oprF, an outer membrane protein, can increase antibody production of the host and allow growth in a microaerobic or anaerobic environment [6, 7, 24].

In Gram-negative bacteria, such as PA, one of the components in the outer membrane is lipopolysaccharide (LPS). The LPS has three parts: an interior lipid A section that is embedded in the outer membrane, a central polysaccharide, and an $\mathrm{O}$ sidechain (antigen $\mathrm{O}$ ). The $\mathrm{O}$ side chain is recognized by a host immune system and can change rapidly in bacteria to evade capture. The lipid A section can be toxic and cause immune responses from the host. In PA, changes to certain genes can alter the $\mathrm{O}$ lateral chain part of the LPS, which helps it remain undetected by the immune system [24].

Virulence often decreases in chronic infections of PA. There is lower production of pyoverdine, pyocins and changes to the type II and type III secretion systems (T2SS and T3SS respectively). This results in less tissue necrosis by PA in a chronic infection than during an acute infection. Loss-of-function of lasR accounts for some of the secretion system changes. LasR is a quorum 
sensing regulator, which activates other virulence factors [6, 24]. Exotoxin genes are also known to be down-regulated or lose function during chronic infection (exoS, exoT, exoY) due to changes in the T3SS which decreases the toxicity of the bacterial cells.

Mutators are often observed in CF-related infections and biofilms. They have fitness advantages in chronic infections due to their ability to rapidly adapt to new environments. Mutator strains can have a 1000-fold increase in the rate of mutation [7]. Changes in the rate of mutation are due to mutations or recombinations in DNA mismatch repair and error detection genes [11]. Environmental factors can elicit this change by prompting microevolutionary changes, such as the presence of damaging reactive oxygen species from from an immune response or the presence of antibiotics. mut genes (especially $m u t L$ and $m u t S$ ), as well as $u v r D$ are often responsible for a mutator phenotype [7]. Although adaptation of PA to a CF lung environment has been studied, we do not yet know all of the mechanisms involved.

\subsubsection{Genome structure of $P$. aeruginosa}

PA has a genome of 5.5 to $7 \mathrm{Mb}$, arranged in a single circular chromosome. The genome contains around 6000 genes, but this varies from strain to strain [37]. A core genome size of around 4800-5200 genes is consistent in the literature [37, 38, 39, 40, 41]. Bacterial strains of the same species do not share $100 \%$ of genes in common, unlike eukaryotes. Thus, the genome is split into two categories, 'core' and 'accessory'. The core genome is that shared by all strains within the species, however this definition is flexible. Usually studies will use a threshold of less than $100 \%$ to account for phylogenetic outliers as well as possible errors with annotation or assembly. The true core genome of bacterial species is widely debated [37, 42, 43]. The accessory genome is comprised of the genes that are shared by some, but not all, strains within a species. The accessory together with the core genome is called the 'pan-genome'.

Several studies have defined the PA core genome as 4.8-5.9 Mbp in length [41, 40, 39, 37]. The pangenome in each of these studies increased as more isolates were sampled [37, 39]. The core genome is comprised of a large number of known metabolic genes (about $80 \%$ of the metabolic genes are in the core genome), cellular signalling and information storage/processing [37]. A number of core genes and most of the accessory genes have an unknown function.

A few other studies have suggested that the core genome harbours fewer than 4800 genes. A 
study using 181 genomes determined a core genome of 2503 genes in $100 \%$ of their strains [43]. A more recent study found an even smaller core genome with 665 genes from 1311 strains [42]. These $100 \%$ cutoffs are too stringent, potentially introducing biases with the samples and sequencing methods used. If a strain is rare and does not have long-term viability but was sampled, genes that are necessary for survival might be excluded. In addition, the dataset would be susceptible to poor assemblies and outliers [44, 45].

PA strains can generally be classified into three groups, based on their reference - PAO1-like, PA14-like, or PA7-like. PAO1 and PA14 are common reference strains that are quite similar [35]. PA7 has been found to be a distant phylogenetic outlier when compared with other PA strains, however it remains in a clade with PA when compared with other species of bacteria [38]. In this strain, robust sections of the genome have been deleted, and there are unique genomic islands.

\subsection{Genome-wide association studies}

Genome-wide association studies (GWAS) have identified causal genomic variants in many human diseases over the past decade and a half [46, 47]. GWAS find correlations between genes or genetic mutations, and diseases. They look for higher incidences of genetic variants in a case (disease afflicted) group compared to a control (non-afflicted) group. Generally these variants are singlenucleotide polymorphisms (SNPs) or copy-number variants (CNVs). GWAS have been successful in identifying genes that contribute to and/or are responsible for type I and type II diabetes, Alzheimer's disease, Parkinson's disease, heart disease, prostate and breast cancer, to name a few [46]. There have been several challenges with GWAS in human studies due to the nature of genomic data. Linkage disequilibrium, population structure and complex traits are the most common concerns affecting GWAS, and have been addressed in various ways over the years.

In human germ cells, chromosomes undergo recombination where homologous chromosomes cross-over and exchange genetic material. This contributes to genetic variation in the population. If two alleles or loci assort independently in a population, they are said to be in linkage equilibrium. Such alleles appear no more often together during sampling than they would when sampled randomly. If two alleles are in linkage disequilibrium (LD), they are found more often together during sampling than expected. This may be because they are located close to each other on a chromosome. During recombination, chromosomes cross-over and exchange genetic material - if 
two loci are far apart, they will often end up in linkage equilibrium in the population. If they are close together, it is more unlikely that cross-overs will occur between them, and they will remain linked for a longer period of time.

LD can be an issue in human GWAS when several alleles/mutations both appear to be significantly associated with a phenotype, but are in high LD with one another. This generally means that not all of them cause the phenotype, but rather that some of the mutations just 'piggyback' during recombination events due to their close proximity on the chromosome. It is important to distinguish these spurious results from the alleles/mutations associated with the phenotype. As a resolution to this issue, the software package PLINK [48, 49] calculates LD between SNPs within a given nucleotide window and gives the option to exclude SNPs that are in LD with one another.

Population structure can be a significant challenge when detecting associations in GWAS. If multiple populations are sampled (for example, a dataset has samples from both European and African genomes), important SNPs may be lost if they only contribute to the phenotype in one population, not both. Conversely, if there are more cases sampled from one population, then variants distinguishing subpopulations may be spuriously associated with the disease. There are many methods that can currently account for population structure and remove these false negatives and positives [50, 51]. Some methods have used principal component analysis (PCA) to account for variation in subpopulations (SMARTPCA [52] and EIGENSTRAT [53]), while others use Bayesian inferences (FastSTRUCTURE [54]) or maximum likelihood models (ADMIXTURE [55]) to infer population structure.

Many human diseases are complex traits, caused by multiple mutations and varying degrees of affliction. When testing for genetic associations to a phenotype, it can be challenging to account for epistasis and interactions between genes [56]. If phenotypes are caused by two-gene interactions, it may be possible to calculate all possible iterations, given time. However with an increased number of interactions and exponential growth in the amount of calculations needed, computational time and resources can be limiting.

Disease-causing SNPs can be rare in a population, resulting in low power to detect associations . In some cases, this can be avoided by using larger datasets. General practice, however, removes variants that are in less than $1 \%$ of the population studied. This decreases computation time and power, as well as removes some false positives. In doing so, true positives may also be inadvertantly 
excluded [56, 57, 58].

\subsubsection{Bacterial GWAS}

In recent years, GWAS have expanded to other organisms, such as plants (e.g. [59]), fungi (e.g. [60]), and bacteria (e.g. [61, 62]). Challenges associated with human GWAS analyses are still prevalent microbial GWAS, but differences between genome and microbial structure can present some unique issues.

Bacteria are predominantly asexual organisms, and usually have haploid circular chromosomes. This differs from humans, who undergo sexual reproduction and have linear, diploid chromosomes. This means there can be long-range LD in bacterial genomes, unlike in human genomes where LD is maintained over short distances. Population structure still plays an important role in bacteria, but can be difficult to determine and verify, unlike in human studies where we can get information about ancestry from a patient to compare against results from population stratification methods. In addition to LD and population structure, genetic drift and natural selection can play a more important role in these large populations with shorter generation times. Genomes are more varied between different bacterial isolates within a species - genes are not always in the same order, and not all genes are shared by all specimens. This can make alignment and genome comparison more challenging.

If bacteria are purely clonal (there is no recombination), the whole genome is in LD. Recombination breaks up this LD, and can vary widely between different bacterial species [61]. Although LD can be accounted for in programs like PLINK [48], if an organism has a highly clonal genome it can still be problematic to find which variants are causal, as found with Mycobacterium tuberculosis [61].

Natural selection can encourage similar populations via positive selection. This can create subpopulations that are genetically alike, but it can be difficult to tell if genome similarity is because of shared ancestry or because of natural selection [61, 63]. Studies have used linear mixed models [63. 64], PCA [65, 66], phylogeny [67, 68], and various programs (e.g. PLINK) to account for population structure.

Another challenge in population structure with bacteria is the possibility of within-host evolution. Some species of bacteria are known to form sub-populations within their host, with some isolates become specialists for their environment, and others generalists, such is the case with PA. A given 
sample may not reflect the diversity within the environment or contain the necessary genetic elements that contribute to an observed phenotype [44]. This is a problem in current research of the microbiome as well, with limited solutions [69].

As mentioned previously, genomes can vary widely between strains of a single bacterial species. This, coupled with higher rates of recombination, can make whole-genome alignment challenging. There are programs that can handle aligning sequences that appear in different parts of a genome (e.g. MAUVE [70]), but performing such alignments is computationally intense with an increasing number of sequences. Bacterial GWAS systems have handled the problem primarily in three ways aligning orthologous genes against each other and using gene presence/absence information in the core genome [65, 71]; using SNP information in just the core genome [61, 72]; or using k-mers [73], where sequences are split into smaller 'words' (10-30bp in length), and these shorter sequences are grouped with other similar k-mers and counted. Each of these methods has strengths and weaknesses - $k$-mers include the most amount of information, but it can be difficult to translate back into gene function; the other two methods may omit information that could include causal variants.

There have been an increasing number of bacterial GWAS published in the last five years, as more whole genomes are being sequenced (Figure 1, Table 1). Each study has addressed challenges, such as population structure or high LD, in different ways. A benefit to bacterial GWAS over human studies is that the results can be explored and verified through genomic manipulation and growth in the laboratory, while in human GWAS it can be very difficult to confirm correlations between SNPs and a phenotype. 


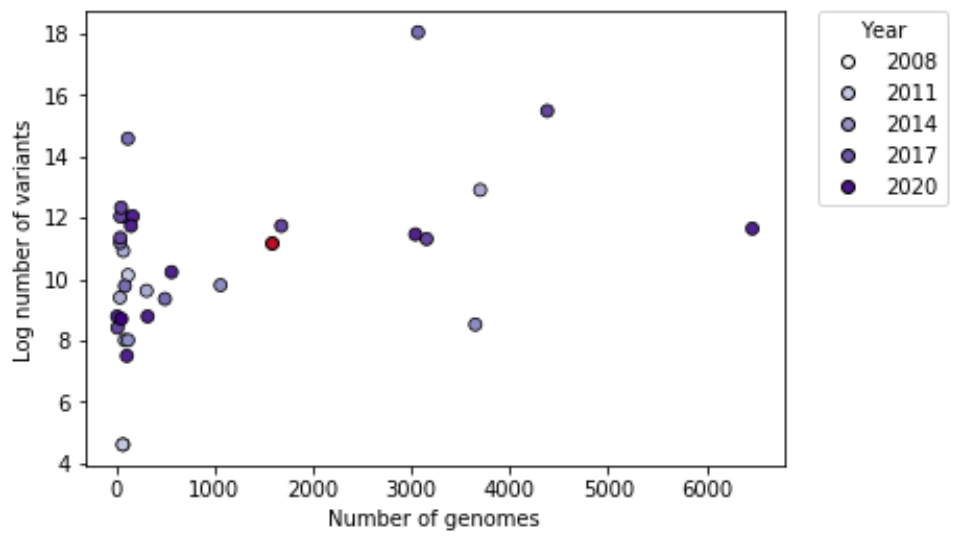

Figure 1: Scatter plot of bacterial GWAS, by year published. Red datapoint is this study. 


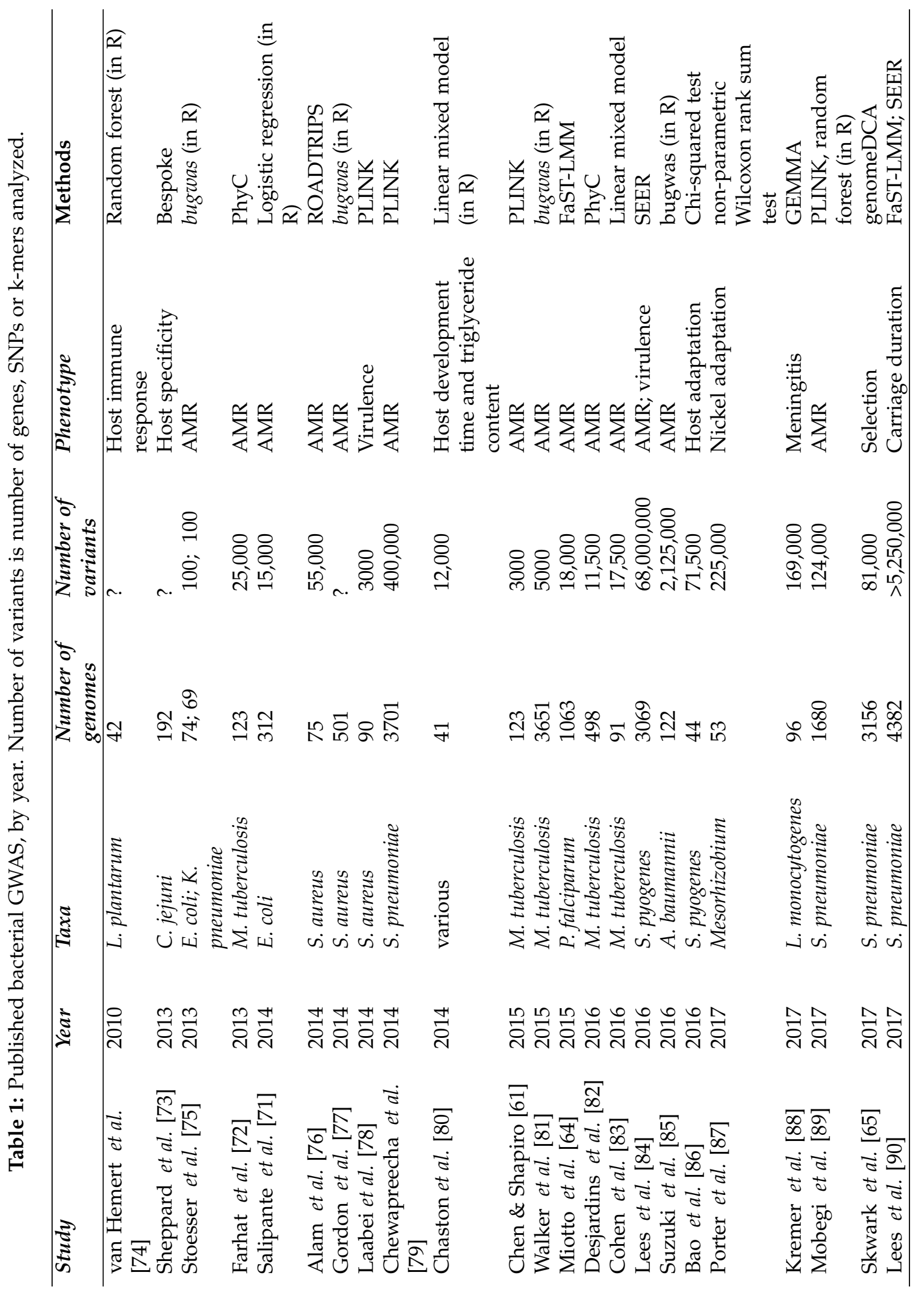




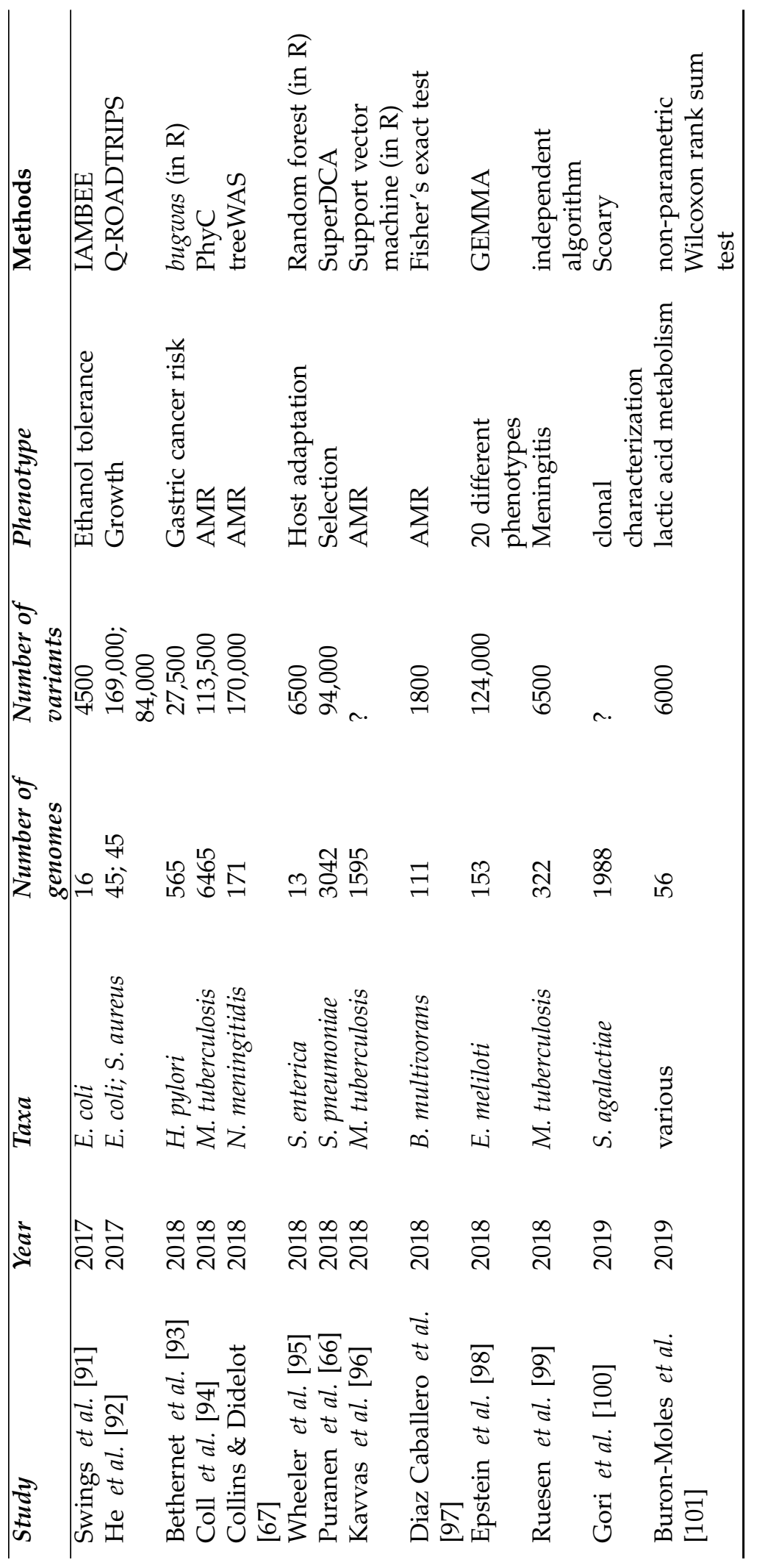




\subsection{Machine Learning}

"Machine learning" refers to a family of artificial intelligence methods that create a model to categorize or classify data points within a dataset. This model can, in some cases, be broken down to determine what features or variables are important for the data classification. This technology is not new, but it has taken a few decades to become more mainstream. It is currently used in many fields, from predicting fraudulent credit card transactions, to recommending advertisements to users, to predicting protein-protein interactions [102].

There are many different algorithms under the umbrella term machine learning, including models such as random forest, support vector machines, neural networks, and k-nearest neighbour. Each method has strengths and weaknesses based on the amount and type of data. For GWAS, we want a classifier that can predict a phenotype and tell us what information was needed to create the model (what features or variants - in this case genetic variants - are important for predicting a phenotype). With supervised machine learning methods, the data are split into training and testing sets, where the algorithm is created knowing all the information about the training dataset, and then that algorithm is tested on the testing data where the phenotype is hidden. An accuracy score is produced based on how often the model was correct for the testing set when compared with the true phenotype. Machine learning methods are particularly valuable for large datasets with a large number of features, where there may be complex solutions that cannot be determined through other methods. Many of these algorithms are very fast, even with thousands of features.

\subsubsection{Random Forest}

Random forest $(\mathrm{RF})$ is a machine learning algorithm that creates a number of decision trees and outputs the mean of the individual trees [103]. A decision tree splits the dataset based on a feature (for example, in a GWAS - presence/absence of a gene) that will maintain the most accuracy in the model. Features that are more basal contribute more to the accuracy of the final prediction. Decision trees take into account multiple features at a time to predict the final phenotype (e.g. presence of Gene A + absence of Gene B + absence of Gene C $=90 \%$ confidence that the individual would be resistant to antibiotics, see Figure 2). Random forests take a group of decision trees, and output the importance of each feature in the model. The importance is either the mean decrease in accuracy over all the decision trees, or the mean decrease in node impurity (measured using the Gini index). 
The Gini index is a calculation of how homogenous the susbets of data are after a given split.

\subsubsection{Support Vector Machine}

Support vector machine (SVM) models are another supervised learning method where the samples are plotted in a multi-dimensional space using all the features available [104]. A hyperplane in that multi-dimensional space is drawn to split the points based on their phenotype, ideally with all cases on one side of the hyperplane and controls on the other. An equation for the hyperplane is calculated, with a coefficient for each feature. The hyperplane coefficient is used to determine which features were most important in splitting the data - the further away from zero the coefficient, the more a variant contributed to splitting the data (Figure 3 ).

\subsubsection{Machine Learning and GWAS}

A large problem with statistical testing is in large $p$, small $n$ problems, where the number of variables tested, $p$, is much larger than the number of samples, $n$. Many machine learning methods can tackle these problems well, by ignoring variables that do not contribute to the model, ie. feature selection. This is perfect for GWAS, where the number of samples is often much smaller than the number of variants investigated.

The adoption of machine learning methods by the GWAS community has been slow, but has gained traction within the last few years. A decade ago, various machine learning models were tried on previously tested datasets, and found similar or improved results with various methods [105]. The main problem encountered with RF and SVM models was the lack of conversion to $p$ values from importance scores or hyperplane coefficients. These models were largely able to find causal variants, even among many irrelevant SNPs. A more recent human GWAS applied an SVM to a previously explored dataset to eliminate variables that are unnecessary to the model, then calculated $p$ values for the remaining SNPs. They found all of the initially discovered SNPs, plus a few that had not been identified in the original analysis [106].

There have been a few bacterial GWAS that have used machine learning methods in the past two years. A study of S. pneumoniae used PLINK [48] to select for genes and SNPs that may confer antibiotic resistance, then used an RF to see if there were any important interactions between them [89]. A different study of M. tuberculosis used an SVM to find genes that contribute to antibiotic 


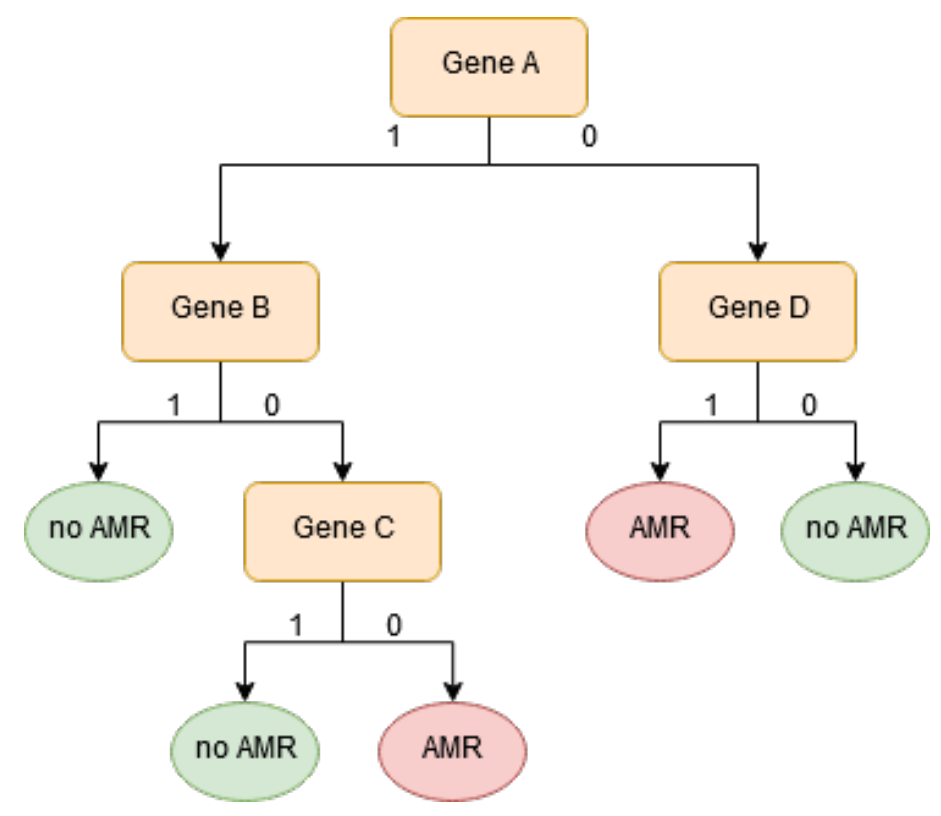

Figure 2: Example of decision tree, where the dataset is split on gene presence (1) or absence (0) to make homogenous groups of antimicrobial resistant (AMR) and non-resistant (no AMR) strains.
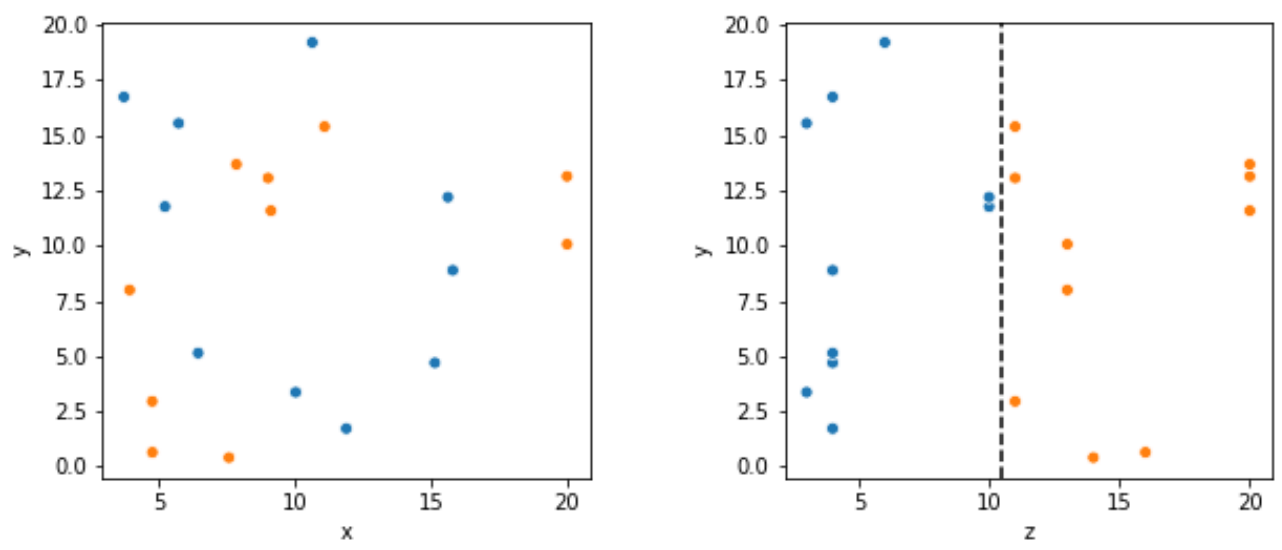

Figure 3: Example of support vector machine model with three variables: $x, y$ and $z$. Strains with phenotype blue and orange cannot be split by $x$ and $y$ (Panel 1), however they can be clearly split on axis $z$ (Panel 2). 
resistance with success [96]. Both studies had around 1600 samples.

Two other recent studies used random forest to look for gene associations in bacteria, however their sample sizes were much smaller. A study of S. enterica had only 13 samples, but was able to identify features putatively contributing to host adaptation. A study of Influenza A and P. aeruginosa had mixed results [107]. They were able to find associations between host adaptation and genes using repeated random forest, with only 10 strains of influenza. However, with only 26 P. aeruginosa genomes, they were unable to converge on an consistent model to find genes associated with AMR. This emphasizes the need for large datasets where possible (hundreds or thousands of samples), even when machine learning algorithms can handle large $n$ small $p$ problems.

\subsection{Motivations}

To find novel genes associated with CF adaptation, I have carried out a genome-wide association study with P. aeruginosa. The goal is to identify potential genes or SNPs associated with adaptation of the bacterium to a CF lung environment. Ultimately, such information may lead to new avenues to treat PA infections. With improved capabilities to treat chronic lung infections, the expected lifespan of a person living with CF can be extended.

I used a variety of approaches to explore which methods perform the best for this, and possibly future, bacterial GWAS. I used a number of different datasets to examine how different datasets and methods affects the results, and identify the most promising gene and SNP candidates in PA for further laboratory research.

\section{METHODS}

\subsection{Data collection and dataset curation}

2225 annotated genomes were obtained from the online Pseudomonas database [1], accessed October 28th, 2017. Strains with missing source information were removed, leaving 1597 genomes. The remaining strains were classified as isolated from $\mathrm{CF}$ patients ("CF" hereafter), or as isolated from other sources ("non-CF" hereafter). The non-CF category thus encompasses isolates from environmental sources and from acute, non CF-related, infections.

Genes in these 1597 genomes had been annotated by various studies using one of 28 reference 
strains (Table 2). I cross-referenced these annotations to identify orthologs between strains, mapping to a reference strain, PAO1. Genes that had no ortholog from annotation were BLASTed against PAO1 genes, using an e-value cutoff of 0.001. If the PAO1 reference hit length was greater than $50 \%$ of the of the query gene length, the match was noted. The longest match with the lowest e-value was identified as the ortholog for each query gene.

Table 2: Reference strains used for annotations in various studies. Known orthologs with PAO1 were used to identify genes in the 1597 genomes.

\begin{tabular}{l}
\hline Strain \\
\hline P. aeruginosa 19BR \\
P. aeruginosa 213BR \\
P. aeruginosa B136-33 \\
P. aeruginosa Carb01 63 \\
P. aeruginosa DK2 \\
P. aeruginosa DSM 50071 - Assembly GCF_001045685.1 \\
P. aeruginosa F22031 \\
P. aeruginosa F9676 \\
P. aeruginosa FRD1 - Assembly GCF_000829885.1 \\
P. aeruginosa LES400 \\
P. aeruginosa LES431 \\
P. aeruginosa LESB58 \\
P. aeruginosa LESlike4 \\
P. aeruginosa LESlike5 \\
P. aeruginosa LESlike7 \\
P. aeruginosa M18 \\
P. aeruginosa MTB-1 \\
P. aeruginosa NCGM1900 \\
P. aeruginosa NCGM1984 \\
P. aeruginosa NCGM2.S1 \\
P. aeruginosa PA1R \\
P. aeruginosa PA7 \\
P. aeruginosa PACS2 \\
P. aeruginosa RP73 \\
P. aeruginosa SCV20265 \\
P. aeruginosa UCBPP-PA14 \\
P. aeruginosa VRFPA04 \\
P. aeruginosa YL84 \\
\hline
\end{tabular}

Those genes that did not have an ortholog in PA01 were clustered using CD-HIT [108] , using a threshold of $90 \%$ identity and default settings. This resulted in 698,710 gene clusters. Clusters containing fewer than 20 genes were removed. Clusters with 19 or fewer genes, even if exclusively found in CF or non-CF strains, would only have a $p$ value of $5.9 \times 10^{-5}$ in a Fisher's exact test, which is below the standard significance threshold of a GWAS, $1 \times 10^{-8}$. This left 10,082 clusters, 
each representing an accessory gene, in addition to 560 accessory genes that were identified in PAO1. Thus, the full pan-genome gene presence/absence dataset consisting of all core (4997) and accessory $(10,642)$ genes, had 15,639 genes (Table 3).

Core genes were aligned against the PAO1 reference, using T-Coffee for translation to amino acid sequences as well as amino acid alignment using default settings. Default settings were used with accurate alignment mode. The number of SNPs for each gene was determined from alignment files. Genes with more than 100 SNPs were manually checked to remove poor alignments. SNPs present in less than $1 \%$ of the population were removed, leaving 54,662 SNPs in 4652 core genes to analyze.

Table 3: Description of datasets analyzed and their size.

\begin{tabular}{llr}
\hline Dataset & Details & Size \\
\hline 1 & Genes in pan-genome & 15,639 \\
2 & SNPs in core genes & 54,662 \\
3 & Genes in pan-genome + SNPs in core genes & 70,301 \\
4 & Genes and SNPs identified with random forest with datasets 1 \& 2 & 561 \\
\hline
\end{tabular}

\subsection{Phylogeny}

A whole-genome phylogeny was inferred using ExaML [109], a parallelized version of RAxML [110], on the Cipres [111] cluster using 1098 core genes. Only genes recovered in 95\% of strains and not found in duplicate in any isolate were used. Gene alignments were performed with T-Coffee [112], using the amino acid sequence and quickaln T-Coffee MSA. Reverse translation was also done using T-Coffee, and DNA sequences for the genes were concatenated to create a single multiple sequence alignment. 11 duplicate strains were removed from the dataset; these strains had exactly the same sequence as another strain. The GTRCAT model with 25 categories and 100 bootstraps was used for maximum likelihood inference, along with Stamatakis ascertainment correction.

\section{3. fastStructure}

FastStructure [54] was performed to infer population structure. FastStructure determines structure within a population using a variational Bayesian framework. Default settings were used, with the number of populations from 1 to 50 , inclusive. 13 populations was found to maximize the marginal likelihood, while 29 populations were needed to explain the structure of the dataset. I 
investigated the makeup of clusters 13 through to 29 to see how many strains belonged to $0,1,2$ or 3 populations. A strain was deemed to be belong to a single population if it was assigned $60 \%$ or more to a population. If there was no population that had an identity of $60 \%$ for a strain, then I counted any population with an identity of over $30 \%$. If no populations had an identity of $30 \%$ or more, then a strain belonged to 0 populations. Only 13, 24 and 26 clusters had 0 strains that belonged to less than 1 population. 24 and 26 clusters required more groupings of populations to explain the structure, therefore 13 populations was determined to best suit the data.

For determining the cluster a particular strain belonged to for analysis purposes, only associations greater than $66 \%$ were assigned. If a strain belonged to a cluster with less than $66 \%$ association, they were assigned no cluster.

\subsection{Association Tests}

\subsubsection{PLINK}

PLINK version 1.9 [48, 49] was used for stratified case/control association, combined with the cluster information from fastStructure. PLINK is often used for human GWAS studies, and has been used in a few bacterial GWAS [61, 79, 78]. PLINK is a whole pipeline that can filter SNP files and determine genetic associations with various tools and algorithms. Default settings were used. LD was also estimated with PLINK, as $r^{2}$ values between SNPs or genes. PLINK was performed on all datasets.

\subsection{2 treeWAS}

TreeWAS [67] is a new program that uses phylogeny to infer genetic associations with a given phenotype. It was created with microbial GWAS in mind, and tries to eliminate the confounding effects of areas in high linkage disequilibrium in the genome, as well as population structure. It has three ways of doing this, though a terminal test, simultaneous test, and subsequent test. The terminal analysis does not require reconstruction of ancestral nodes in the phylogeny, but rather relies on the terminal state. The simultaneous analysis looks at the probability of changing phenotype and genotype states along a branch, is not impacted by missing associations, and can infer relationships involving multiple changes along a branch. The subsequent analysis calculates the probability of finding the same genotype and phenotype on each branch. 
Association scores are reported for each analysis. These association scores are calculated by first creating a null distribution of association scores using a simulated dataset, where there is no association between a loci and the phenotype. The association between each loci is then measured and calculated, following the same distribution of the null association scores. Scores and loci at the upper end of the null distribution are reported.

TreeWAS was used with each dataset and one of the bootstrap phylogenies created with ExaML. Strains that had been removed by ExaML due to sequence similarity were re-introduced to the tree, sharing a node with the strain their sequence perfectly identified with.

\subsubsection{Machine Learning Methods}

Random forest (RF) and support vector machine (SVM) classifiers were performed on each dataset of 1586 P. aeruginosa strains, using the scikit-learn package in Python 3.6. Random forests were run with 10 decision trees, and the maximum possible number of features for each dataset. Support vector machines were run with a linear kernal.

\subsubsection{Other Statistical Tests}

Fisher's exact test and principal component (PC) analysis were carried out using Python package scikit-learn. All boxplot comparisons of PCs were output and analyzed. Only PCs with significant differences between $\mathrm{CF}$ and non-CF strains were plotted against each other.

\section{Dataset Results}

If a gene was in $95 \%$ of strains, it was identified as a core gene (Figure 4 resulting in a core genome dataset of 4997 genes. Strains missing more than 10\% of core genes were removed from the dataset. This left 1586 strains for analysis. 


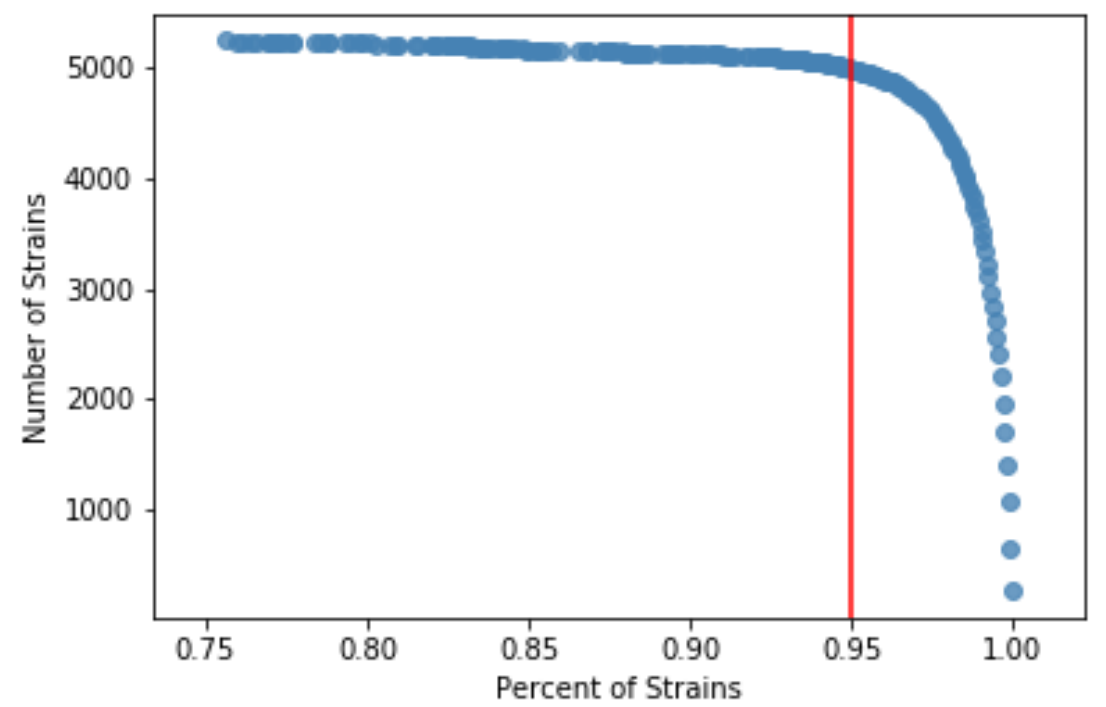

Figure 4: Number of PAO1 genes present in proportion of strains in the dataset. A 95\% threshold was used for the core genome - if a gene was in $95 \%$ or more of strains, it was labelled as a core gene. 4997 genes were part of the core genome. 


\subsection{Phylogeny}

I inferred a maximum-likelihood phylogeny for all 1586 genomes using ExaML [109]. CF and non-CF strains are interspersed throughout the tree. This implies that the CF strains do not all have shared ancestry, and that CF infections represent multiple, independent evolutionary events (Figure 5). Furthermore, for most studies that contributed more than one strain, strains are spread out around the tree (studies that contributed over 100 strains and that were not found in a single clade are highlighted). Only 2 studies that contributed more than 40 strains were found to be in a monophyletic clade. The leaves highlighted in green are from a study of the Liverpool epidemic strain. The other highlighted leaves, in orange and pink, were from a longitudinal studies of PA samples from single patients, one in Toronto (orange) [16], the other in Italy (pink) [17]. This phylogeny was subsequently used to ensure that positive associations were not due to shared ancestry alone, and to analyze the datasets using a phylogeny-based GWAS method, treeWAS [67].

\subsection{Population structure}

Population structure may confound GWAS analyses: if affected individuals tend be drawn from the same population, then non-causal variants that happen to occur at a higher frequency in that population may falsely be associated with the disease. As such, I used FastStructure [54] to infer population structure in this dataset.

Cluster proportion identified from FastStructure can be seen in Figure6. There are three clusters of just CF strains, one of which contains 220 strains which were all collected from a single patient. Three more clusters are composed of $95 \%$ or more CF strains: one of them contains all of the Liverpool epidemic strains, along with one non-CF strain. Two clusters are a 35/65 split of CF to non-CF strains. Only one cluster is $95 \%$ or more non-CF strains. One cluster has no members that are associated with it fully. 436 strains ( 287 non-CF, 149 CF) are not assigned to a cluster with $66 \%$ or more membership.

Phylogenetically, the FastStructure clusters are not monophyletic, but several of the clusters have all members in close proximity within the tree. 


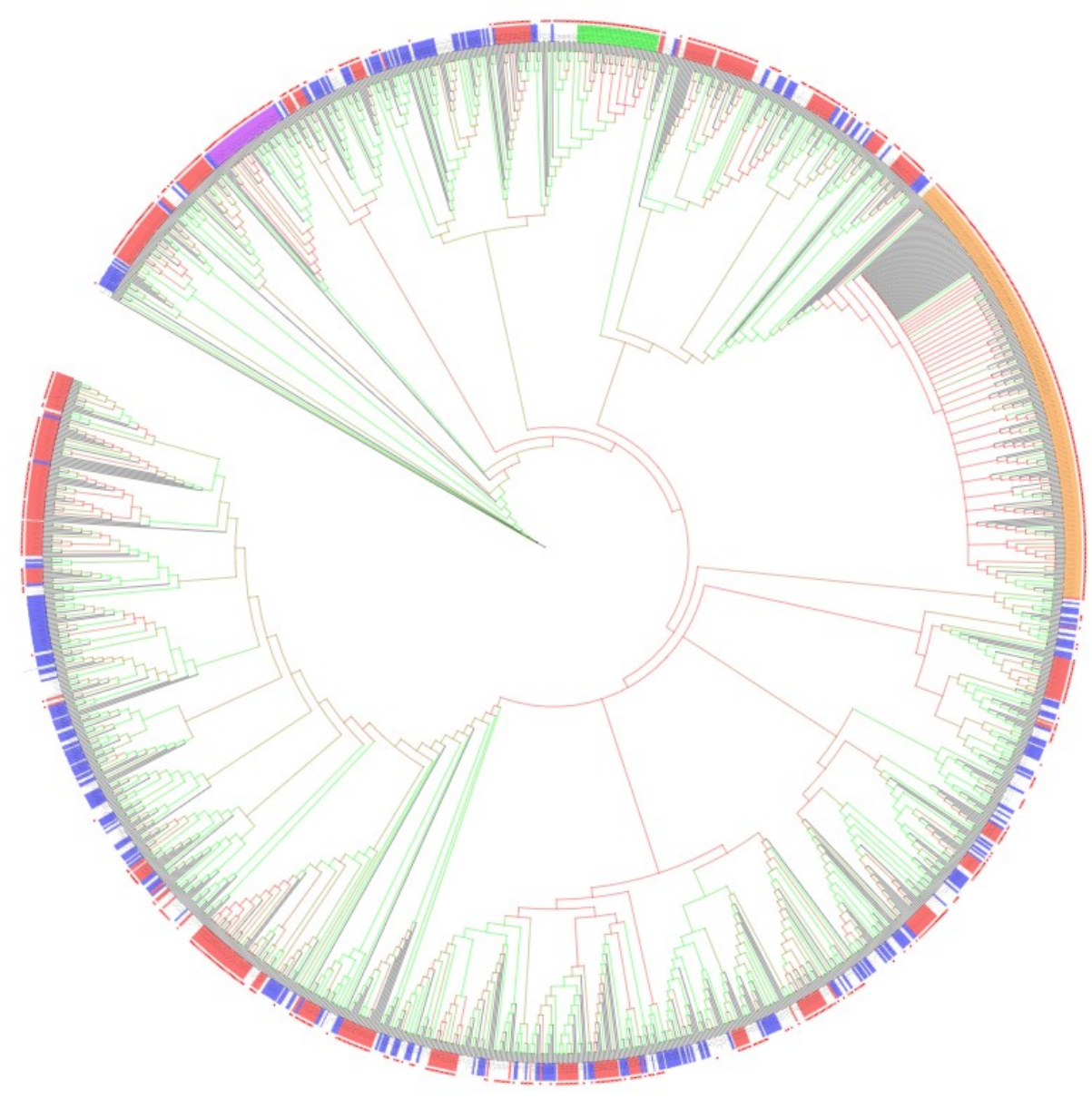

Figure 5: Phylogeny of 1576 P. aeruginosa genomes. Red squares outside the tree denote CF strains. Studies contributing $>100$ strains are highlighted (red and blue), as are studies with $>40$ strains found in a single clade (orange, pink and green). Branch colours show bootstrapping confidence, green is 100 , red is 1 . 


\section{GENE PRESENCE/ABSENCE DATASET RESULTS AND DISCUSSION}

\subsection{Linkage Disequilibrium}

In addition to population structure, high linkage disequilibrium can confound association results. I examined LD in the gene presence/absence dataset (Figure 7) using PLINK. Aside from a few short sections, there is little linkage between genes. In P. aeruginosa there is little LD beyond $3 \mathrm{~kb}$, and there is a rapid decrease in LD after about 200bp [113]. Since most genes in the accessory genome are separated by more than $1 \mathrm{~kb}$, this explains why the figure shows little LD.

\subsection{PLINK}

I carried out a case-control association analysis in PLINK, using 13 population clusters to attempt to control for population structure. A quantile-quantile (QQ) plot was generated as a quality control measure. A QQ plot shows the distribution of calculated $p$ values against a uniform distribution of expected $p$ values. The QQ plot (Figure 8 ) shows that the calculated $p$ values are substantially higher than the expected $p$ values. This suggests that there are many type I errors (false positives) in the PLINK results. Ideally, we would want to see the datapoints mostly follow the red line (which represents a uniform distribution of $p$ values), with a deviation near the tail where there are more significant associations. There could be some population structure not accounted for by fastStructure, which is often the case in human GWAS with QQ plots that show an excess of type I errors.

Even though the QQ-plot is not what I was ideally looking for, the PLINK association tests gave interesting results. It identified 145 genes as contributing to a CF phenotype, over 13 clusters or families (genome significance cutoff of $1 \times 10^{-8}$ ).

Figure 9 shows a Manhattan plot of the genes tested in PLINK, and is usually a way to visualize significant genes in a study. In an ideal plot, "spikes" of points will appear, with some of the points in these spikes above the significance threshold. What this indicates, biologically, is that there is a gene/SNP at this location that is associated with the phenotype studied. Although there will be often be several points above the significance threshold in a given spike, some of these other points may appear to be associated with the phenotype due to high LD between it and a causal variant. It is the researcher's job to try to keep this in mind, and try to identify the causal variant 


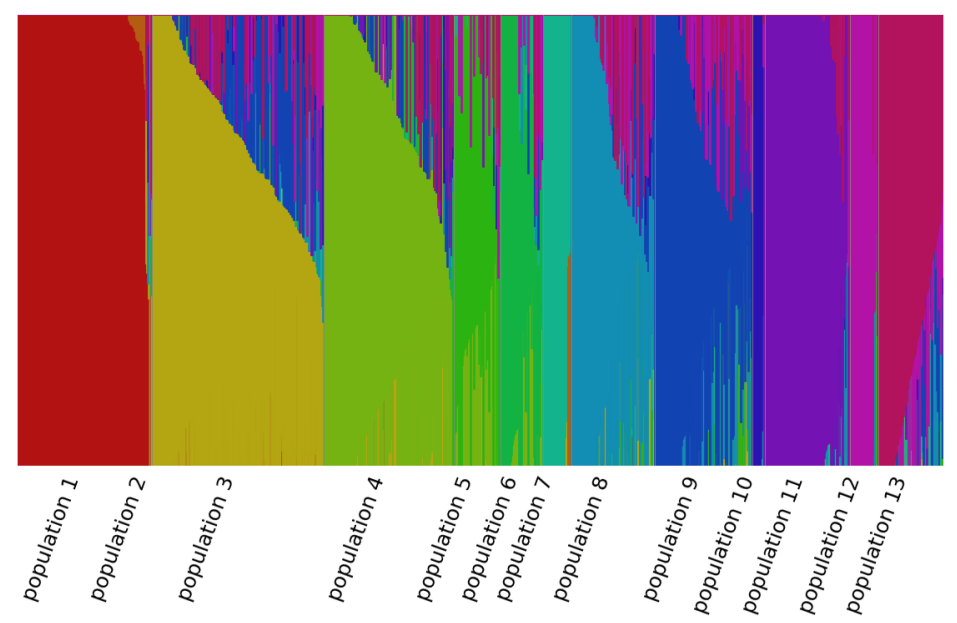

Figure 6: Proportion of cluster association for all strains. The x-axis is the strain, ordered by population. The $y$-axis is the proportion of belonging to a cluster.

Figure 7: Linkage disequlibrium pattern in P. aeruginosa genes, $r^{2}$ as calculated in PLINK. The x-axis represents the location along the pan-genome. The first 3513 locations are ordered as in strains from Table 4. The last 6000 positions are ordered as found in PAO1. 


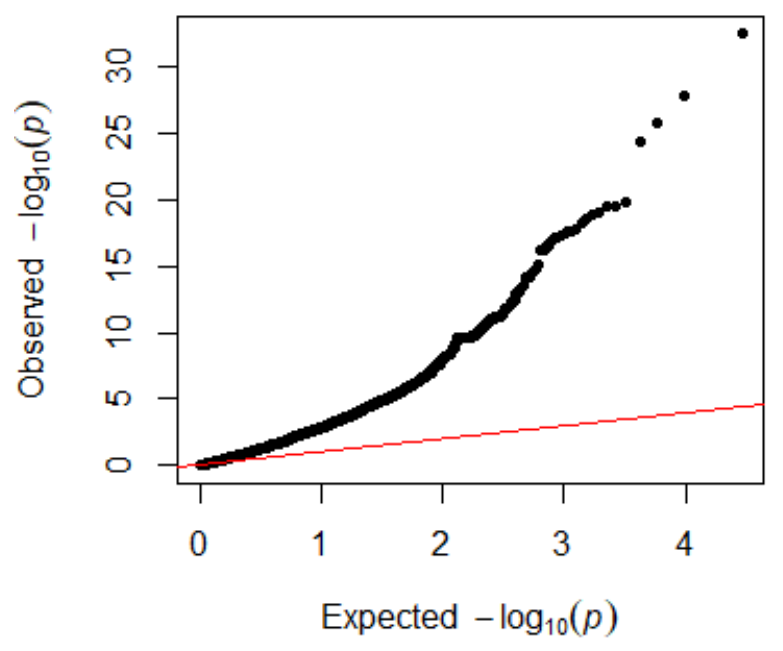

Figure 8: QQ plot of calculated $\log p$ values in PLINK of the gene dataset against expected $p$ values. The red line is when the observed $p$ values equal the expected $p$ values $(\mathrm{x}=\mathrm{y})$.

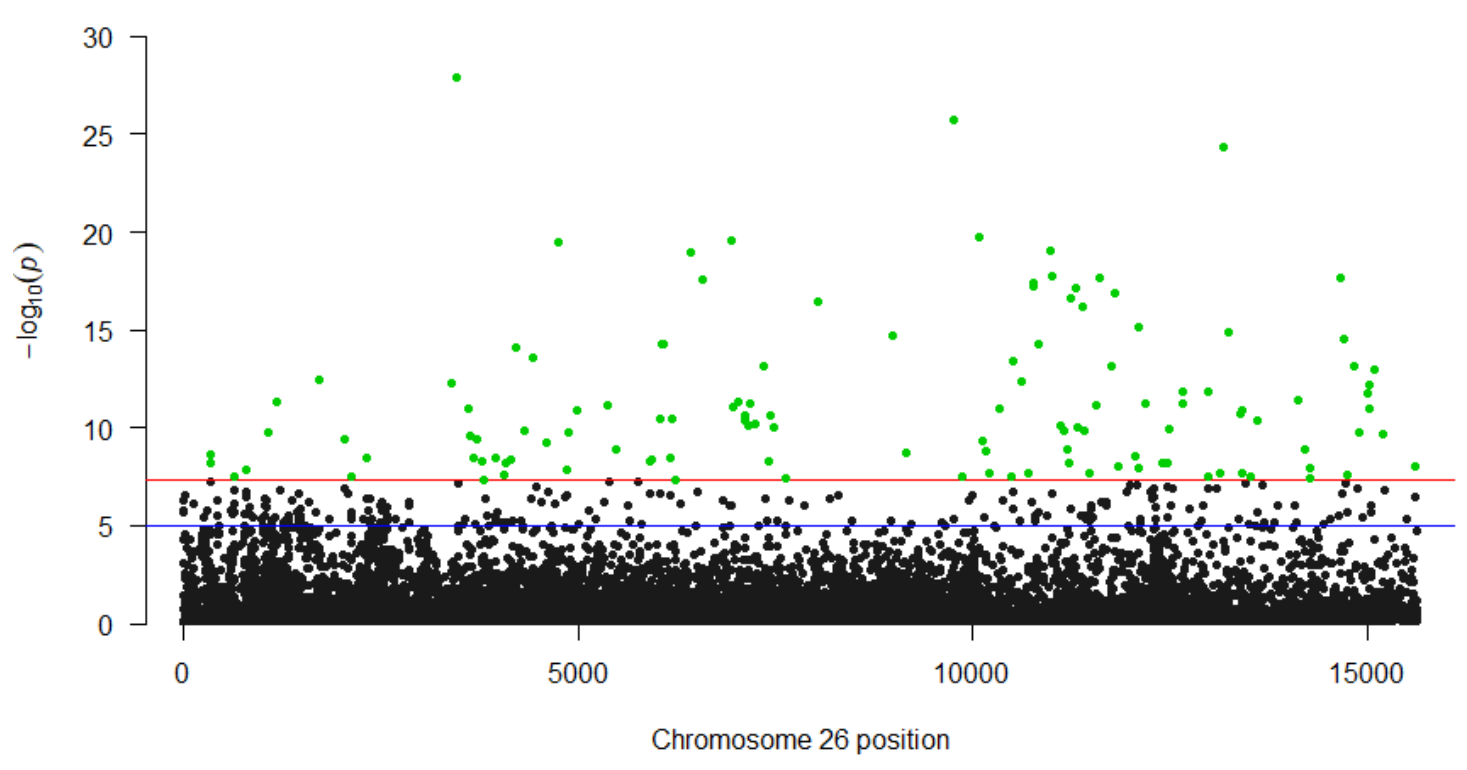

Figure 9: Manhattan plot of $p$ values in the PLINK analysis of the gene dataset. Points above the red line are significant, and coloured in green. The red line is the genome-wide significance line, at $\log \left(1 \times 10^{-8}\right)$. The blue line is the suggestive significance line, at $\log \left(1 \times 10^{-5}\right)$. 
from the non-causal variants in these spikes. The difference between Figure 9 and a Manhattan plot from a human GWAS study is that all genes are in the same order in humans, and so the plot would ideally show spikes in certain areas (resembling a Manhattan skyline, hence the name). However, in bacteria, genes are in different orders in each strain, so a standard sequence and spikes in certain areas can be unattainable. Any points above the line represent genes with significant associations (in green). Although the order for the first 3000 genes are as they are found in a single strain (Table 4), and the last 5000 genes are ordered as in PAO1, there is little linkage between genes with significant associations. This is due to a lack of LD, since I found little long distance LD in the dataset (Figure 7) - there are few genes that are found in combination with each other consistently, no more than if random assortment were to occur.

Table 4: Strains used for ordering of accessory genes. Only reference genomes with known orthologs to PAO1 that had 100 or more unaccounted for accessory genes were used. Ortholog information was retrieved from the online Pseudomonas database [1]

\begin{tabular}{ll}
\hline Strain & Number of accessory genes \\
\hline NCGM1984 & 768 \\
VRFPA04 & 517 \\
LES400 & 457 \\
19BR & 436 \\
PA7 & 286 \\
SCV20265 & 239 \\
PACS2 & 231 \\
UCBPP-PA14 & 180 \\
PA1R & 153 \\
F22031 & 140 \\
MTB-1 & 106 \\
\hline
\end{tabular}

Many of top genes found by the PLINK association tests are hypothetical proteins or pseudogenes. The characterized genes in the top 40 results are listed in Table 5 . Several of these genes have known functions.

ton $B$ genes encode for outer membrane receptor proteins that are involved in iron-acquisition. tonB2 was the second iron acquisition gene found in the PA genome. In recent years, iron acquisition has been shown to play an important role in bacterial CF phenotypes. Ferrous iron is abundant in CF sputum [114]. There is controversy over whether virulence of PA is impacted in an iron-rich or ironpoor environment, and the amount of iron in a CF lung environment [114, 115, 116, 117, 118, 119]. Furthermore, tonB2 was found to be a determinant for gentamyacin resistance in a small-scale GWAS that looked for associations between genes and antimicrobial resistance [107]. Iron transport 
Table 5: Top genes from from PLINK stratified association analysis

\begin{tabular}{lll}
\hline Rank & Gene & $\boldsymbol{p}$ value \\
\hline 1 & tonB2 & $3.09 \times 10^{-33}$ \\
5 & recF & $1.66 \times 10^{-20}$ \\
12 & two-component response regulator & $1.63 \times 10^{-18}$ \\
16 & pdtA & $4.08 \times 10^{-18}$ \\
17 & hxcY & $6.46 \times 10^{-18}$ \\
20 & napA & $2.62 \times 10^{-17}$ \\
24 & mexZ & $6.61 \times 10^{-16}$ \\
25 & oprB precursor & $1.38 \times 10^{-15}$ \\
30 & mucA & $5.73 \times 10^{-15}$ \\
31 & mutL & $7.61 \times 10^{-15}$ \\
33 & mexB & $3.61 \times 10^{-14}$ \\
36 & enolase-phosphatase E-1 & $7.61 \times 10^{-14}$ \\
\hline
\end{tabular}

systems have been looked at as a possible method to tackle antibiotic resistance [118], something which is abundant in P. aeruginosa infections in CF patients. Looking at the dataset, a large majority of CF strains are missing tonB2 (768/947 missing, over $80 \%$ of CF strains), while only about half are missing this gene in non-CF strains $(347 / 639)$.

RecF is a non-essential DNA replication and repair protein that has been found to be involved in recombination, but has not been linked to a CF phenotype. It has, however, been shown to have some importance in Ciprofloxacin resistance in a small study that used a random forest [107], although the authors readily admit that their results are questionable due to such small training sets. $r e c F$ is overwhelmingly found in strains that are not from a CF source $(578 / 639,90 \%$ of non-CF strains), but presence and absence of the gene is equally split among CF strains (444/947 contain the gene, or $47 \%$ ).

$p d t A$ encodes for phosphate depletion regulated TPS partner A, a protein involved in a twopartner secretion system that increases virulence of PA strains [120, 121]. Limiting inorganic phosphate activates the expression of $p d t A$. The mechanism of increasing virulence is unknown, but it was demonstrated that $p d t A$ mutants are less virulent than wild-type strains with a functional $p d t A$ in a C. elegans killing assay [120]. $p d t A$ was present in only $85 \%$ of CF strains (812/947), but $97 \%$ of non-CF isolates $(623 / 639)$.

$h x c Y$ encodes a type II secretion system protein, but its function has not yet been researched. napA is a periplasmic nitrate reductase protein which has been found to be upregulated in Liverpool epidemic strains when compared with PAO1 [122] and could be a possible immunogenic protein 
[123].

Mutlidrug efflux pumps (Mex proteins) confer antibiotic resistance by pumping out drugs and other materials from the cell [124]. The role of efflux pumps in antibiotic resistance in CF PA strains has been widely studied [30, 125, 126]. mexZ encodes for a well characterized protein that confers aminoglycoside and fluoroquinolone resistance [127, 128, 129]. MexZ regulates the MexXY multi-efflux pump, and is often found mutated in P. aeruginosa coming from CF lungs. In the dataset, the gene is missing in more CF strains than not (missing in 749/947 strains, 79\%), while it is more present than not in non-CF strains (missing in only 249/639 strains, 39\%). The absence of MexZ would leave MexXY unrepressed, increasing the bacterium's resistance to antibiotics by more effectively pumping out drugs. mexB encodes for a different efflux pump that confers resistance to beta-lactams, tetracyclines, trimethoprim, and fluoroquinolones [130]. Similar to mexZ, mexB was absent more often in CF strains (missing in 80/947, 8.4\%, missing in only 14 non-CF strains, 2.2\%), however the discrepancy between CF and non-CF strains was not as large. It is not immediately clear why mexB might be more often absent in CF strains, as it encodes for an efflux pump; it is not just a regulator like MexZ.

The outer membrane porin protein $\mathrm{OprB}$ has been characterized as a carbohydrate transfer protein in PA [131]. When combined with a knockout of bpeAB in Burkholderia pseudomallei, however, it confers broad spectrum drug resistance [132, 133, 134]. bpeAB have not been found in PA, and this link between oprB and antibiotic resistance has not been yet been demonstrated in the species. It is however possible that a similar mechanism of action exists in PA. Alternatively, since the CF lung presents a unique environment for PA, it is possible that oprB is not as useful for carbohydrate transfer as in other environments. Looking at the correlation between the presence/absence of the gene in CF and non-CF strains, the gene is missing in roughly a third of CF strains (329/947), but is only missing in $14 \%$ of non-CF strains $(92 / 639)$.

Deletion of $m u c A$, a gene identified as contributing to the CF phenotype with PLINK association analysis, has been shown cause mucoidy in P. aeruginosa [15, 135]. A mucoid characteristic is common in $\mathrm{CF}$ infections. MucA is part of the AlgU pathway, which encodes RNA polymerase sigma-H factor. When the algU pathway is dysfunctional (either due to missing or defective algU, $a \lg D$ or $m u c A$ genes), the strain becomes mucoid [135, 136]. This helps the strain become established in the lung and adapt to the host's stress response [6]. Looking at its occurrence in CF and non-CF 
strains, the gene is missing in more than half of CF strains (only 431/947 strains have the gene, $45.5 \%$ ), while only 45 non-CF strains were missing the gene (gene is present in 594/639 non-CF strains, $93.0 \%$ ). This could be because the absence of $m u c A$ in a non-CF strain leads to a substantial loss of fitness in other environments.

mutL encodes a protein that is part of the mismatch repair system. Issues with DNA repair can lead to more mutations within the genome and increase the rate of adaptation. MutL loss-offunction in bacteria has been shown to promote large-scale chromosomal deletions through double strand breaks and promote hypermutation [137, 138]. mutL was present in 887/947 CF strains (93.7\%), and almost all non-CF isolates (634/639, 99.2\%).

\section{3. treeWAS}

TreeWAS scores genes based on their association to a phenotype while avoiding clonal associations (associations based on identity by descent). It corrects for population structure differently than PLINK, using the phylogeny and phenotype to look for significant gene associations that are not confounded by shared heredity. It incorporates different models, calculating associations when there are phenotypic state changes along branches, or not. With the simultaneous model (ie. changes in genotype and phenotype were allowed along a branch), association scores with an absolute value larger than 19 were deemed significant, identifying 129 genes of interest (Table 6. Figure 10). Well over half of the genes (74/129) were hypothetical proteins, however, and many of the top genes were unexpected from previous research into infectious PA strains. The top genes that have known functions were also found and described in the PLINK section (Section 4.2), such as tonB2 and recF.

Table 6: Top genes found in treeWAS combined association analysis.

\begin{tabular}{lll}
\hline Rank & Gene & Association Score \\
\hline 1 & protease & -50 \\
2 & hxcY & -49 \\
3 & PA1539 & -49 \\
4 & PA1729 & -48 \\
5 & napA & -46 \\
7 & tonB2 & -45 \\
9 & recF & -43 \\
13 & PA2074 & 37 \\
\hline
\end{tabular}




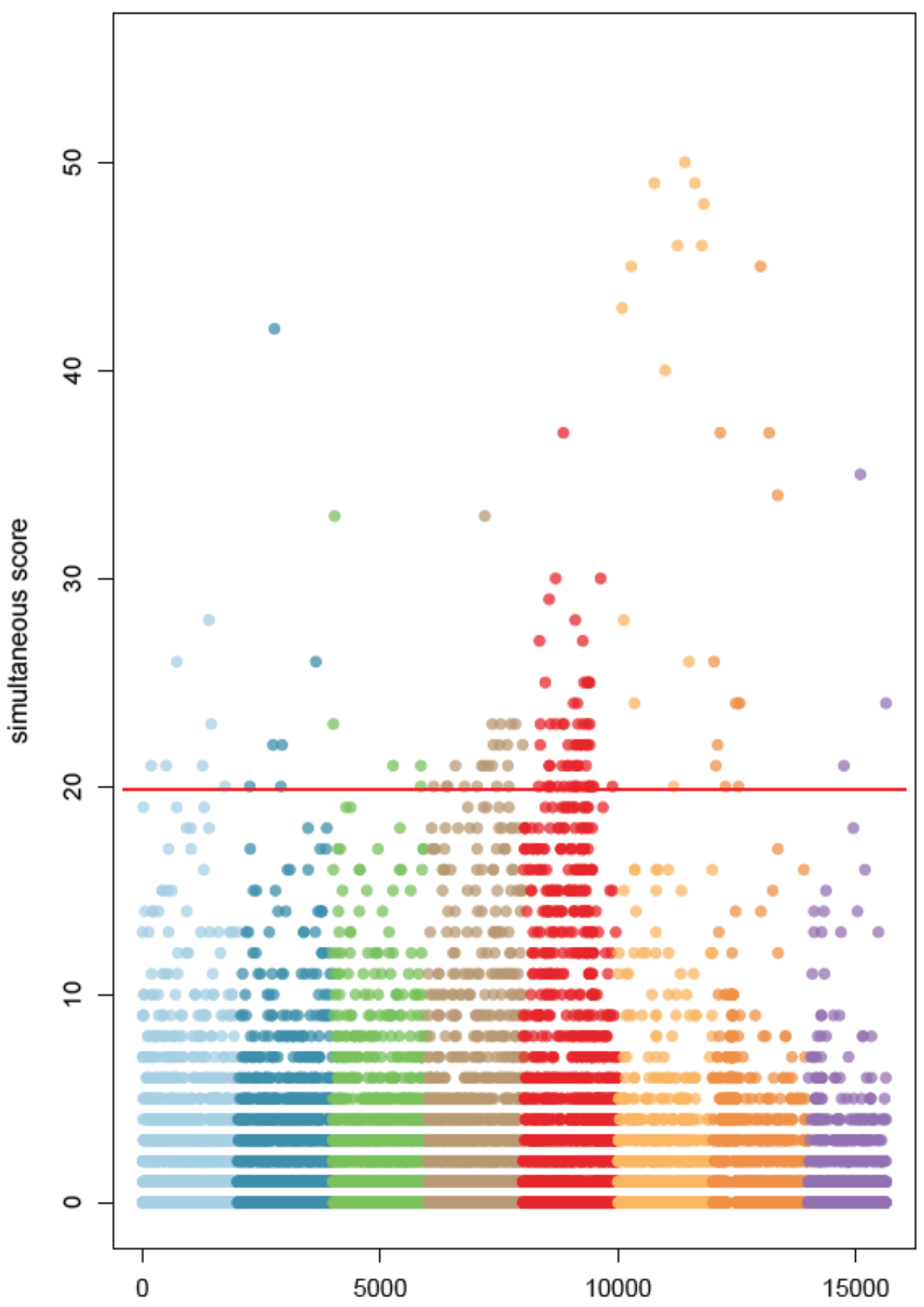

Figure 10: Manhattan plot of gene scores found from treeWAS association simultaneous analysis. 


\subsection{Random Forest}

Random forests create a set of decision trees that look to accurately predict the phenotype using as few variants as possible. They use the information from these decision trees to create an overall importance metric, which identifies the variants that are the best predictors of the phenotype. The model had $93.4 \% \pm 1.0 \%$ accuracy when predicting $\mathrm{CF}$ versus non-CF strains when given presence/absence information for about 265 genes (Table 7). This means that knowing the presence or absence of 265 genes, the model could correctly predict a whether a strain was CF or non-CF 93.4 out of 100 times. Several of the top genes identified by random forest are already corroborated in literature, lending support to the model. Many of the top genes were also found in the PLINK and treeWAS tests (Sections $4.2 \& 4.3$ ). The top hit from random forest, PA2074 (unknown function) was also identified in the treeWAS analysis in the top 15 genes.

Table 7: Top genes that contribute to the random forest model.

\begin{tabular}{lll}
\hline Rank & Gene & Importance \\
\hline 1 & PA2074 & 0.237 \\
2 & mucA & 0.123 \\
3 & tonB2 & 0.084 \\
4 & PA5037 & 0.041 \\
5 & recF & 0.029 \\
8 & mexF & 0.011 \\
10 & efflux pump & 0.009 \\
11 & mutL pseudogene & 0.009 \\
\hline
\end{tabular}

The random forest approach identified $m u c A$ as being important to the CF-phenotype within the top 5 genes, which was not the case with PLINK nor treeWAS. PLINK did identify mucA as the 30th most important gene contributing to a CF phenotype, while treeWAS did not identify it at all.

mexF is a gene that encodes for an efflux pump and contributes to antimicrobial resistance in various bacterial strains, similar to mexB and mexZ described in section 4.2 mexF was present in a higher proportion of non-CF strains compared to CF strains (present in only 66\%, 626/947, of CF strains; present in 98\% non-CF strains, 626/639).

Other named genes with known functions that contributed to the RF model were identified in the earlier PLINK section (Section 4.2).

I carried out several additional tests with RF to assess the effects of potential confounding factors. For example, genes were dropped that were identified in only one study. Only 77 genes fit this 
criteria, none of which were found to be important in the original model, and removing them did not change the results. In another test, genes that had a presence/absence scheme that was $95 \%$ similar were collapsed to a single gene cluster. As such, all core and a few accessory genes (5096 genes total) were combined into 1 cluster. This new model needed only 148 genes to determine the difference between $\mathrm{CF}$ and non-CF strains, and did so with $93.8 \% \pm 1.0 \%$ accuracy. 46 genes identified in the first RF model were combined into the 'core' cluster, and therefore did not appear in the new model. 97 genes were identified that were not in the first model, and 51 genes appeared in both models (Appendix C.1).

\subsection{Support vector machines}

An SVM was able to separate the CF from the non-CF strains with $94.0 \%$ accuracy. Unlike with an RF, an SVM does not output a smaller set of important genes/features, but rather every gene has a coefficient in the hyperplane, and it is up to the researcher to determine what the cut-off is. A strongly negative or positive coefficient for the hyperplane contributes more to the model than values that are close to 0. As can be seen in Figure 11, the distribution of coefficients resembles a log-odds curve when values are plotted from lowest to highest. Most coefficients are around 0 , with only 510 genes lower than -0.02 or higher than 0.02 . Of the genes with the highest absolute hyperplane coefficient, most of the top six genes are recognizable and expected (Table 8). mucA and tonB2 were among the most important genes identified in the RF model as well.

The phenazine biosynthesis protein PhzE is a catalyst for synthesizing virulence factors, such as pyocyanin and phenazines in P. aeruginosa [139]. It has been shown to be overexpressed in burn victims and underexpressed when grown in the blood of healthy patients (when compared to growth in LB broth) [140]. In CF patients, strains that produce auto-antibodies against bactericidal/permeability increasing protein (BPI-ANCA) cause infections that are more difficult to treat. These strains are found to produce fewer phenazine derivatives. Strains without BPI-ANCA, in vitro, produced more phenazine derivatives, and induced a higher immune response in epithelial cells [141]. In this dataset, the gene is more often absent in CF strains (only present in 349/947 strains).

Unfortunately, after the top six genes, many of the genes with high absolute coefficients are hypothetical proteins or do not stand out as contributing to a CF phenotype. 196 out of 504 
remaining genes with absolute hyperplane coefficients greater than 0.02 encode for hypothetical proteins, without counting pseudogenes.

Table 8: Top genes found in support vector machine model.

\begin{tabular}{lll}
\hline Rank & Gene & Absolute hyperplane coefficient \\
\hline 1 & mucA & 0.08961 \\
2 & oprB precursor & 0.0875 \\
3 & mexZ & 0.0727 \\
4 & phzE & 0.0654 \\
5 & PA2727 & 0.0619 \\
6 & tonB2 & 0.0584 \\
\hline
\end{tabular}

\subsection{Fisher's exact test}

I performed a Fisher's exact test on each gene, as this is a common statistical method used in other GWAS [142]. A Fisher's exact test simply asks whether a gene is more often present (or absent) in $\mathrm{CF}$ vs. non-CF isolates. One challenge in performing a Fisher's exact test is determining the significance threshold. Over 1000 genes had $p$ values less than $1 \times 10^{-20}$, and around 200 genes had odds ratios of infinity (meaning that given the presence of that gene, there was an infinite chance the strain was from a CF patient). Given such low $p$ values, even after a Bonferroni correction ( $p$ value significance cutoff is divided by the number of variables, so any $p$ value $<3.2 \times 10^{-6}$ is deemed significant), thousands of genes were over- or under-represented amongst CF strains of PA. The Manhattan plot shows thousands of genes to be significant (Figure 12); 1000 genes is too many to test in a lab in a reasonable amount of time. The odds-ratio was also unhelpful, as many of the top genes were only observed in one study, which suggests that the results are due to artifacts from sequencing or proximity of strains and are not due to the strain adapting to a cystic fibrosis lung environment. Without more samples, the Fisher's exact test is unable to provide valuable insights. Furthermore, with a standard Fisher's exact test, I am not able to correct for population structure, something that other GWAS studies do, which could make fewer genes significant to a CF strain of PA.

\subsection{Principal component analysis}

Principal component analysis (PCA) is a method of dimensionality reduction whereby many dimensions and the data they describe are "summarized" by a smaller number of vectors which 


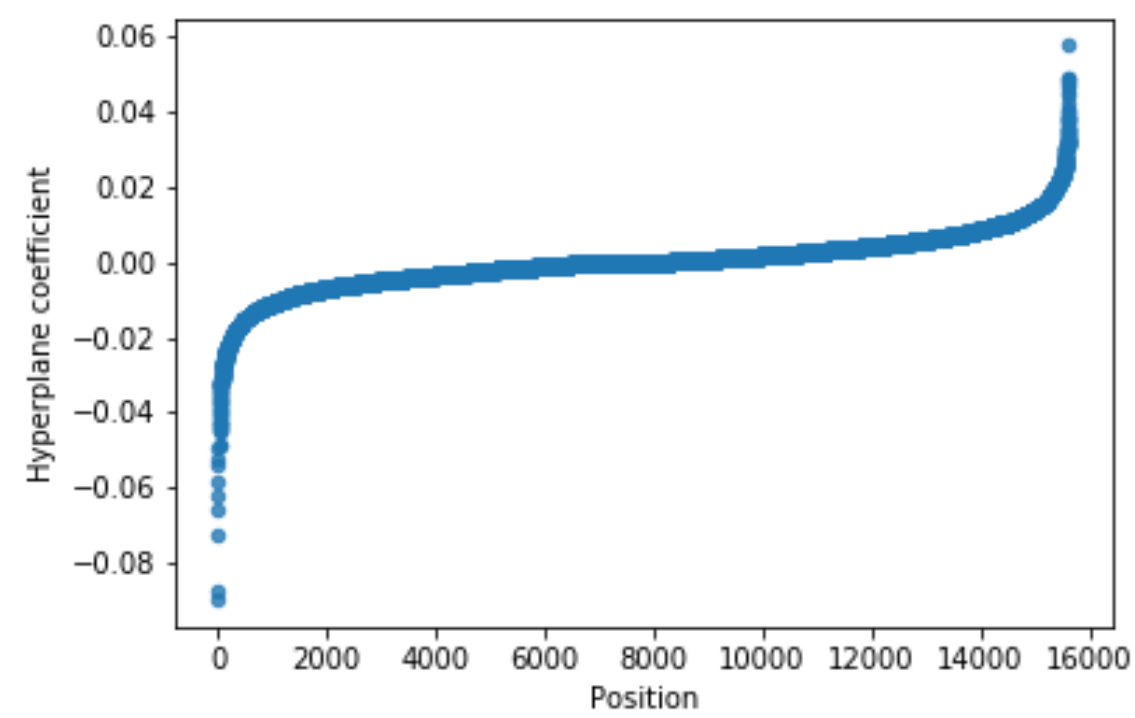

Figure 11: Hyperplane coefficient from linear support vector machines. Gene index was sorted based on lowest SVM coefficient to highest, to show the spread of values.

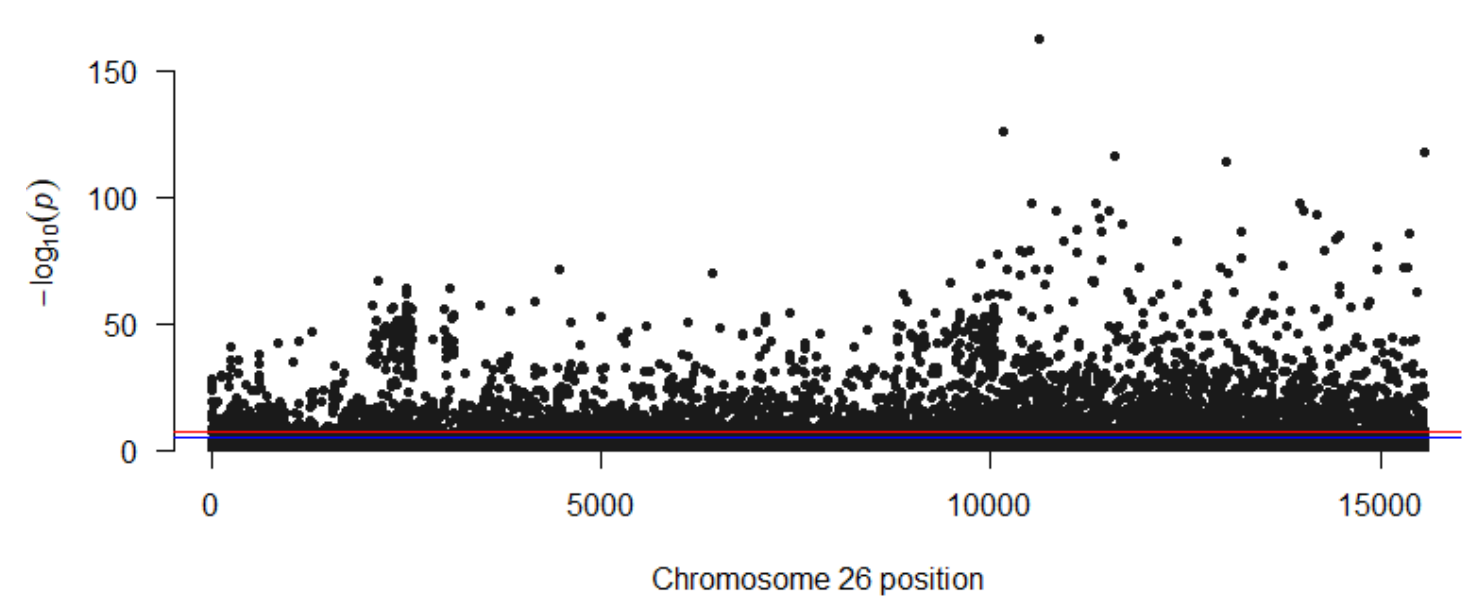

Figure 12: Manhattan plot of $p$ values from a Fisher's exact test for each gene found in any of the 1600 tested P. aeruginosa strains. The red line is the genome-wide significance line, at $\log \left(1 \times 10^{-8}\right)$. The blue line is the suggestive significance line, at $\log \left(1 \times 10^{-5}\right)$. 
capture the greatest differences in those feature dimensions and thus reduce the number of dimensions. This is performed at the cost of losing those minimal differences between dimensions and operating in a new, smaller feature space that no longer contains exactly the original dimensions. PCA was performed on the dataset to see if, after a reduction in features, there were any components that separated the CF strains from the non-CF strains. There were 54 components that accounted for $50 \%$ of the variance between different strains. PC 1 did not differentiate CF from non-CF strains, however PCs 2 and 3 did (Figure 13). A plot of PC2 against PC1 (Figure 14) was able to show two clusters, one that was primarily CF strains (orange), and one that was mostly non-CF strains (blue).

The primary genes that make up principal components 2 and 3 are hypothetical proteins. None of the top 10 genes in either component (either strongly positively or negatively correlated with the component) are strongly supported in the literature. Genes that would be expected to be of importance (antibiotic resistance genes, metabolism or mucoidy genes) contribute little to the components. These results could be taken into account when looking at other machine learning results, however they are not promising on their own.

\subsection{Conclusion of gene presence/absence dataset}

There are some genes that were found consistently using PLINK, treeWAS, RF and SVM (Figure 15. tonB2 was recovered using each of the four methods, within the top 10 genes identified. $h x c Y$ was also found in each of the methods, however was not strongly significant in three of the four methods. A few hypothetical proteins were also identified with these four methods: PA5037, an unnamed non-ribosomal peptide synthetase, and an undefined hypothetical protein.

Some of the genes highly cited in literature were identified in three of four methods, such as mucA, mexB and mexZ, mutL, and recF (Figure 16). The top genes identified using a random forest had the fewest hypothetical and most corroborated genes in literature. Though this model found the largest number of genes as contributing to a CF-phenotype, most of these genes had little importance (0.001), and could be excluded when identifying which genes should be further researched. 

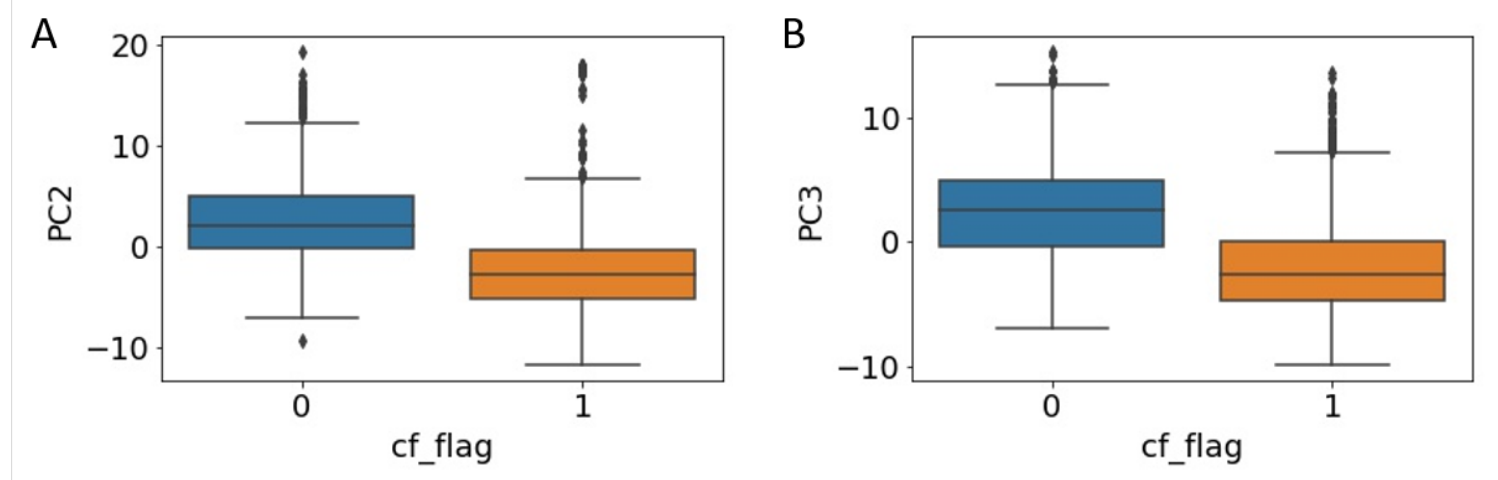

Figure 13: Boxplot of mean principal component values of genes for cystic fibrosis and non-cystic fibrosis strains. CF_flag of 1 is a cystic fibrosis strain, CF_flag of 0 is a non-cystic fibrosis strain. A) Principal component 2 mean values, B) principal component 3 mean values. 

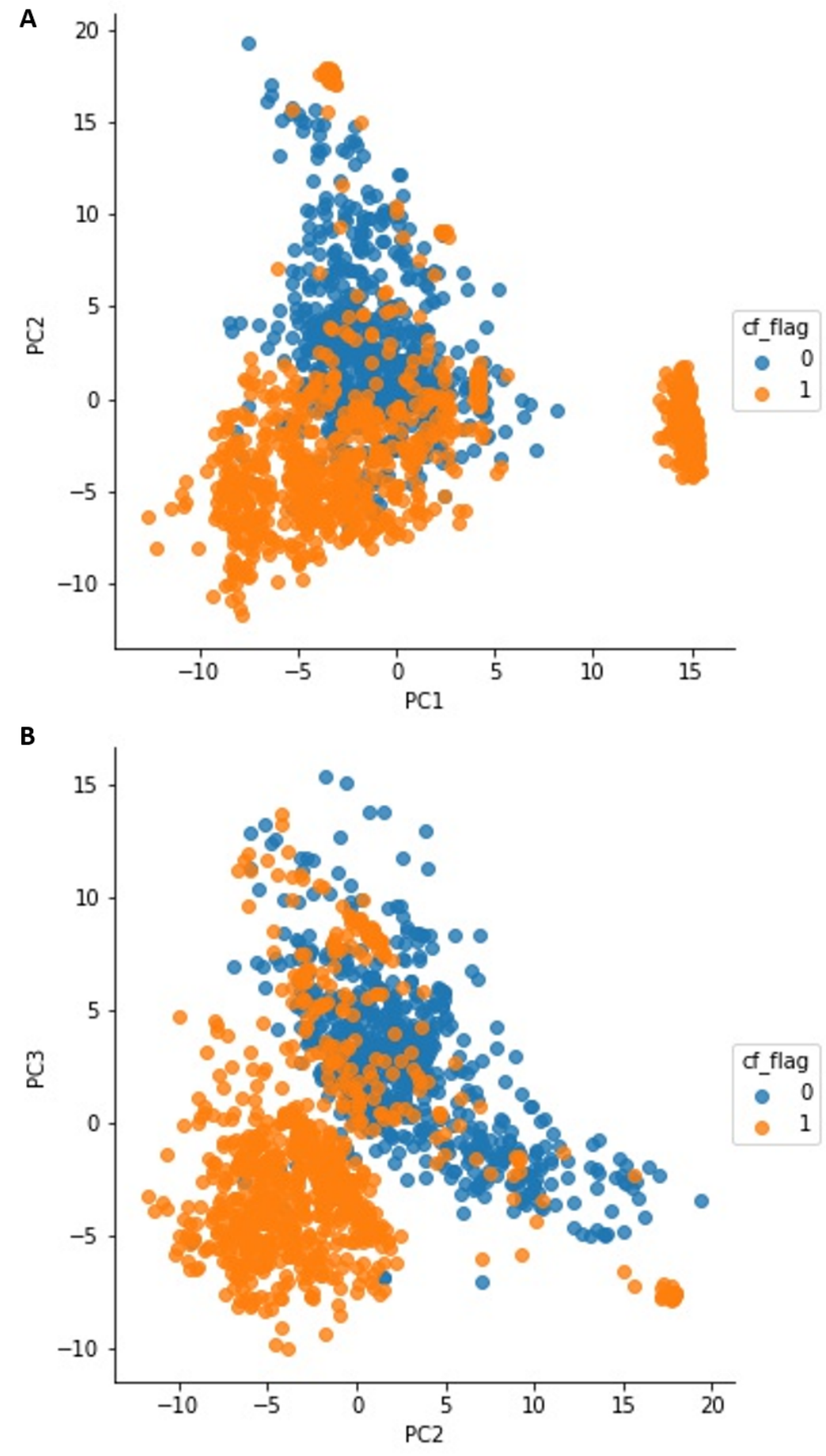

Figure 14: Plot of principal component 2 values against principal component 1 for genes of cystic fibrosis and non-cystic fibrosis P. aeruginosa strains. 


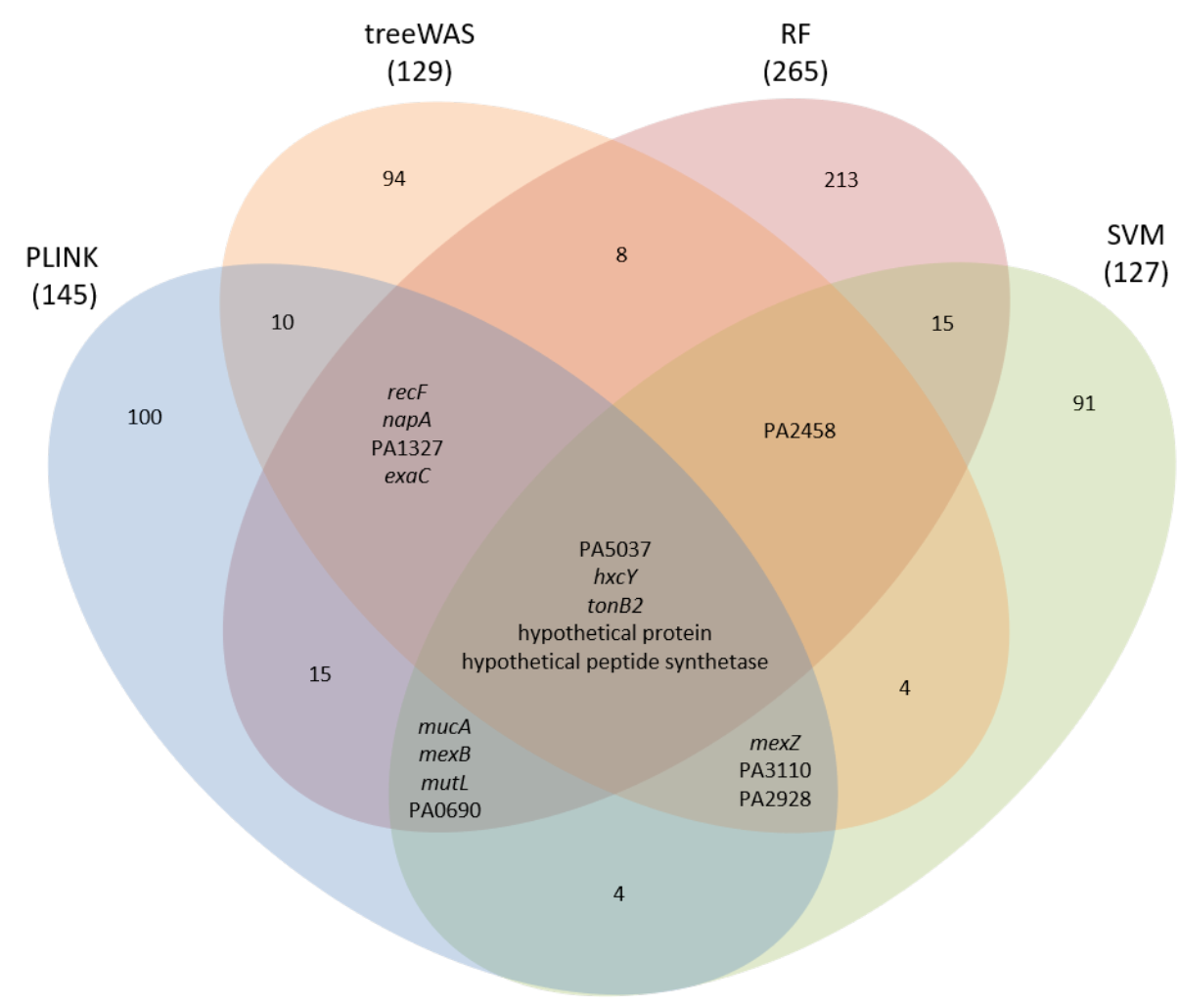

Figure 15: Venn diagram of results from PLINK, treeWAS, random forest and support vector machine. Genes identified in at least 3 of the 4 methods are reported.

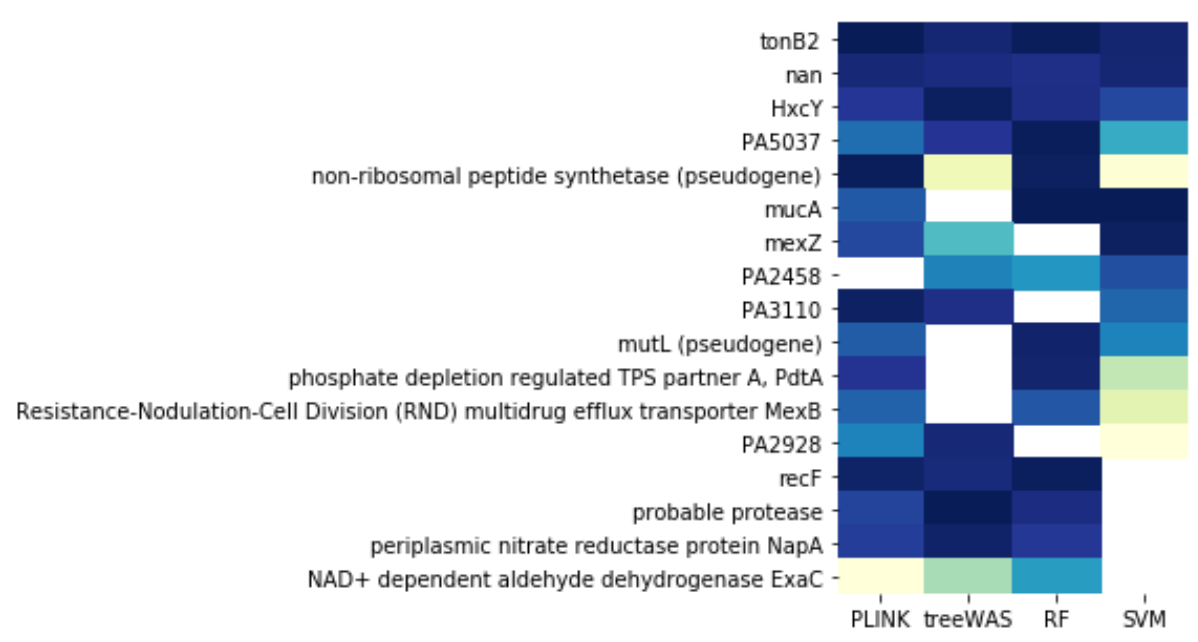

Figure 16: Heatmap of genes found in the gene presence/absence dataset with various methods. Darker blue is a higher ranking (lower $p$ value or higher association score/ importance/ hyperplane coefficient) gene. Yellow is a lower ranking variant. White is not identified as contributing to the phenotype. 


\section{Mutation DATASET RESUltS AND DisCUSSION}

\subsection{Linkage Disequilibrium}

LD was tested with the SNP dataset using PLINK, using default settings. LD was higher between SNPs within the core genome than between genes in the pangenome (Figure 17). LD drops off rapidly, within the first few kilobases (Figure 18), which is consistent with previous research [113]. The hotspot of LD about halfway in the genome includes several metabolism and transport genes, such as podL and mexE, along with many unclassified genes (80 of 111 genes in that area are hypothetical). The highest mean $r^{2}$ value was around $500 \mathrm{bp}$, at 0.7655 . A $50 \%$ reduction in this LD was found after just $700 \mathrm{bp}$, with an $r^{2}$ value of 0.3811 .

\subsection{PLINK}

I carried out a PLINK case/control association study for the SNP dataset using the populations from fastStructure with the gene dataset. This gave a QQ plot with the lowest deviation from the expected $p$ values. The QQ plot (Figure 19) with the SNP dataset shows a larger deviation from the expected $p$ values than the gene dataset (Figure 8). Nonetheless, there is still a marked deviation from the expected uniform distribution.

The Manhattan plot (Figure 20) does not have spikes, as might be expected from a human GWAS Manhattan plot. There are many SNPs that were identified in PLINK as contributing to a CF lung environment adaptation.

Associations identified in the PLINK analysis include SNPs in several genes with known or suspected roles in adaptation to the CF lung, while others are not known to be involved in CF pathoadaptation (Table 9). For example,the ATP-binding component of an ABC transporter has been suspected to contribute to ciprofloxacin (antibiotic) resistance [143]. The deletion at position 269 was in only $320 / 947$ CF strains (33.8\%), but 400/639 non-CF strains (62.6\%).

PqqF is a required protein for PQQ (pyroquinolone quinone) biosynthesis. The elimination of pqqF results in the inability of the bacterium to produce prodigiosin from glucose [144, 145]. The H12R mutation is present in $80 \%$ of CF strains (767/947), while it is only present in $26 \%$ of non-CF strains (170/639). The H3R mutation has similar statistics, present in 761/947 CF strains, but only $165 / 639$ non-CF strains. The $r^{2}$ value between these two mutations is 0.965 , meaning I 


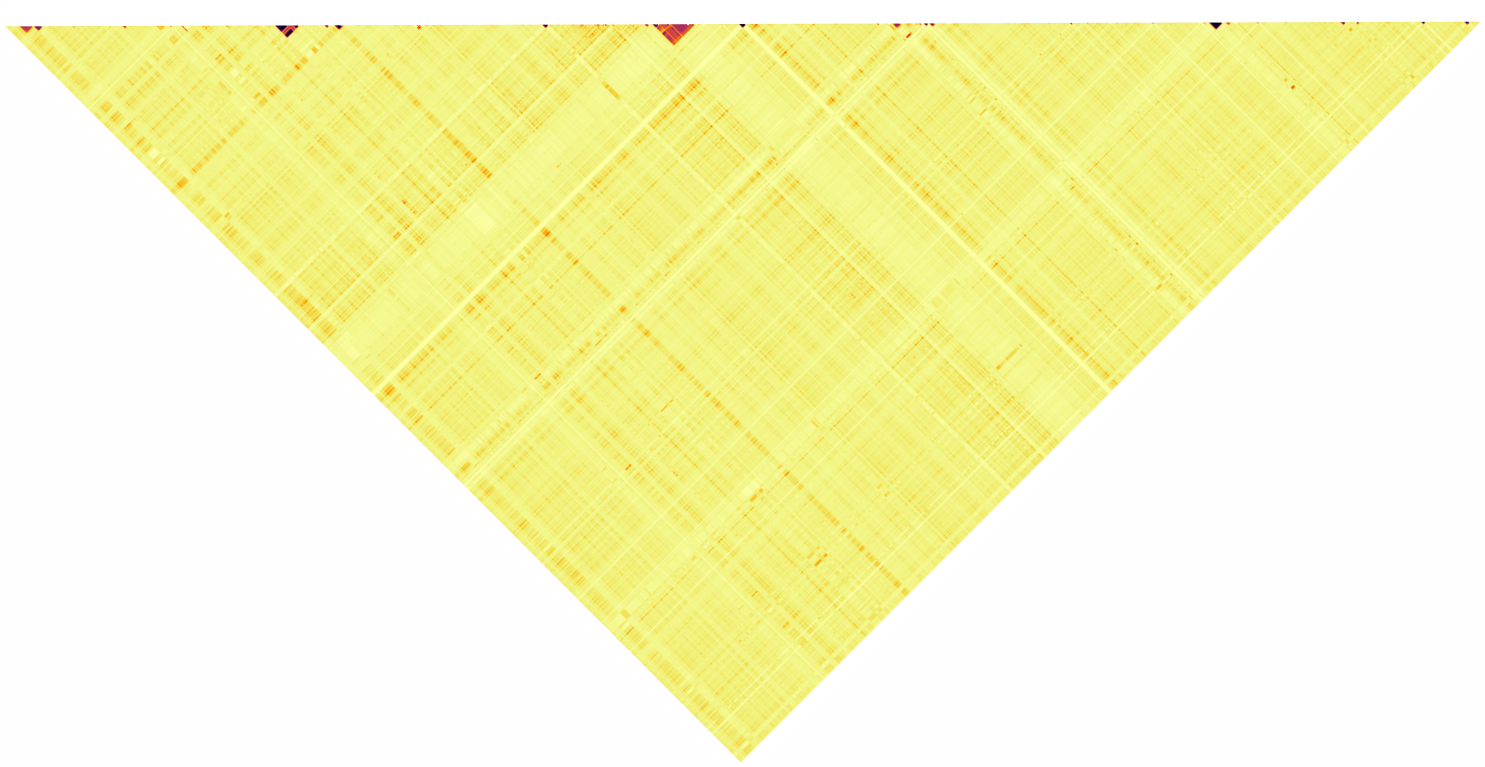

Figure 17: Linkage disequlibrium pattern in P. aeruginosa core genome mutations, as calculated in PLINK. The $x$-axis represents the location along the PAO1 genome.

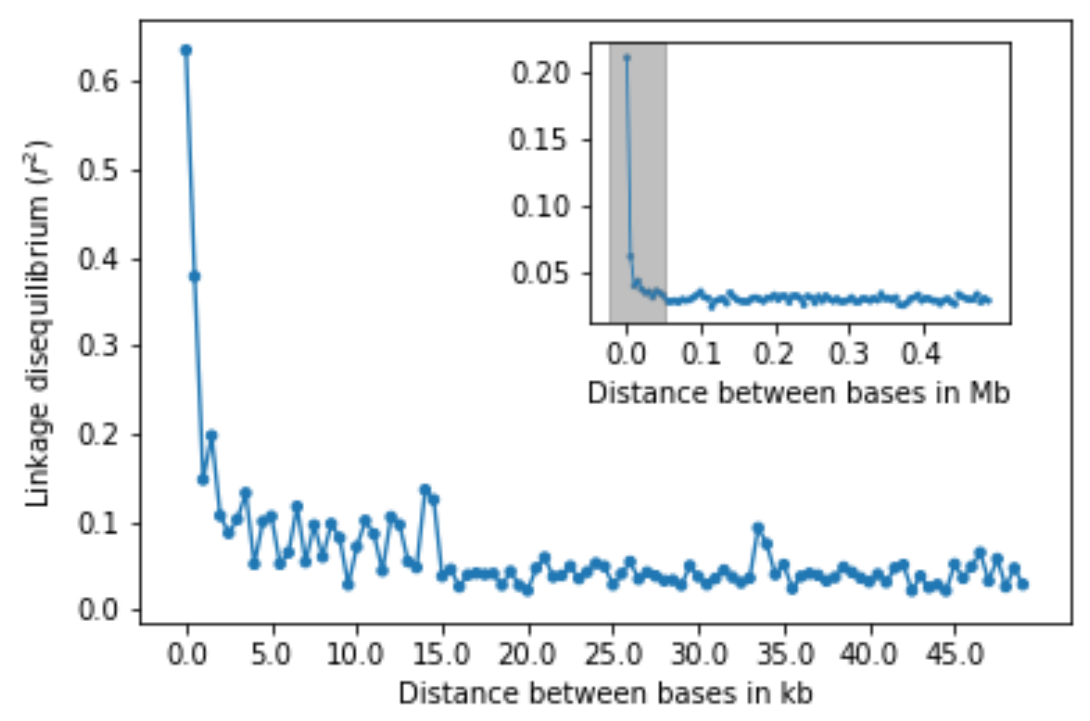

Figure 18: Linkage disequlibrium $\left(r^{2}\right)$ decay in P. aeruginosa. 


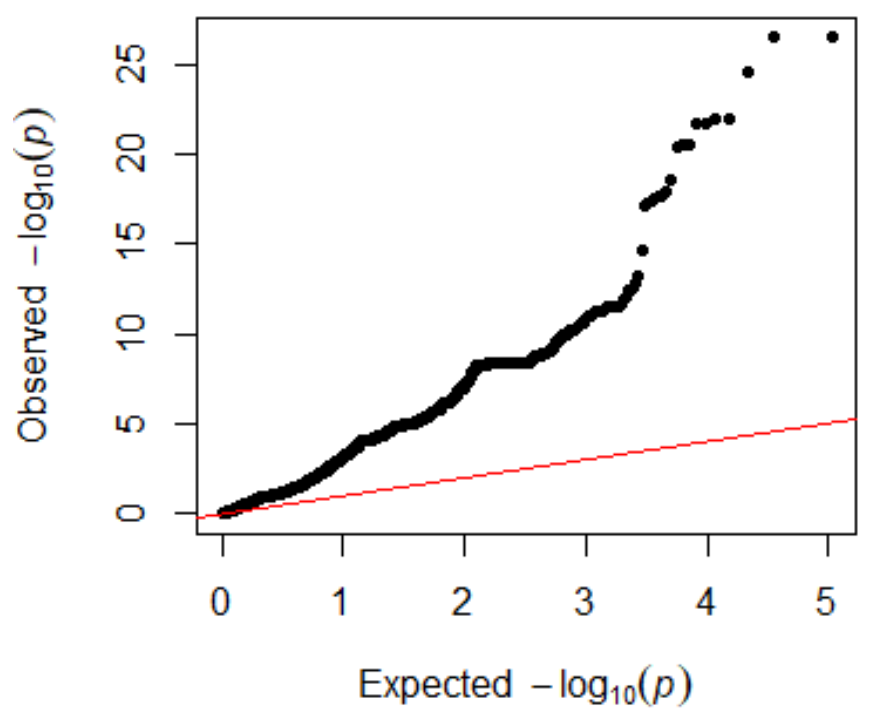

Figure 19: QQ plot of calculated $\log p$ values in PLINK of the mutation dataset against expected $p$ values. The red line represents when observed $p$ values equal the expected $p$ values $(\mathrm{x}=\mathrm{y})$

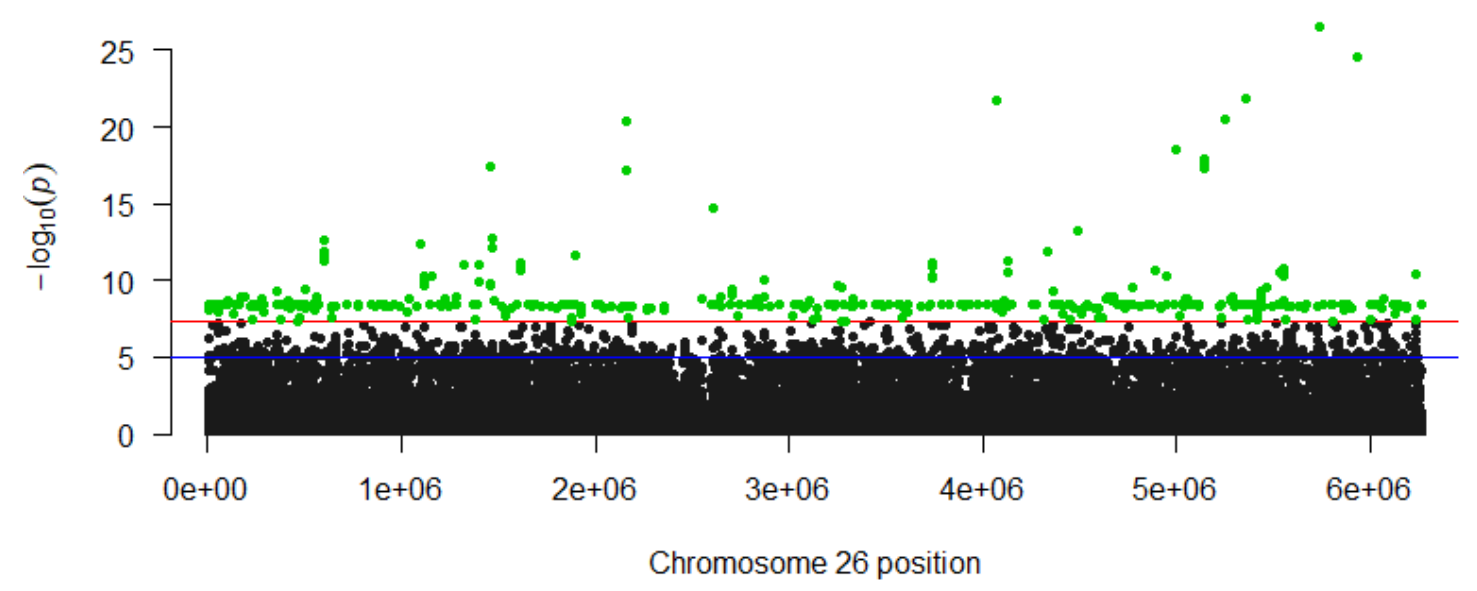

Figure 20: Manhattan plot of $p$ values in the PLINK analysis of the SNP dataset. Points above the red line are significant, and coloured in green. The red line depicts the genome-wide significance line, at $\log \left(1 \times 10^{-8}\right)$. The blue line represents the suggestive significance line, at $\log \left(1 \times 10^{-5}\right)$. 
Table 9: Top SNPs and indels from from PLINK stratified association analysis

\begin{tabular}{llll}
\hline Rank & Gene & Mutation & p value \\
\hline 1 & probable ATP-binding component of ABC transporter & 269 deletion & $3.32 \times 10^{-27}$ \\
6 & $p q q F$ & H12R & $4.31 \times 10^{-21}$ \\
8 & $p q q F$ & H3R & $6.54 \times 10^{-18}$ \\
10 & proA & R275Q & $6.07 \times 10^{-14}$ \\
12,13 & wapB & D12S, S24A & $3.97 \times 10^{-13}$ \\
15 & narG & A144V & $1.26 \times 10^{-12}$ \\
16 & cysH & L2P & $2.54 \times 10^{-12}$ \\
19 & $i c i A$ & M1L & $2.51 \times 10^{-11}$ \\
\hline
\end{tabular}

cannot determine which mutation is causal since they are in high linkage disequilibrium.

ProA is a gamma-glutamyl phosphate reductase that is involved in proline biosynthesis. There is no research to show that this is involved in the adaptation to a CF lung environment or that it contributes to antibiotic resistance. The mutation at position 275 is more common in non-CF isolates (present in 167/947 CF strains, 17.6\%, and 259/639 non-CF strains, 40.5\%).

$w a p B$ is a newly characterized gene in PA. It is involved in the outer core biosynthesis of lipopolysaccharide (LPS) in the cell membrane [146, 147]. The LPS is a major virulence factor that affects the inflammation and immune response of the host [148]. Both mutations have similar prevalence among CF and non-CF strains. D12S is present in 263/947 CF strains (27.8\%), while S24A was found in $271 / 947$ (28.6\%). Both mutations were in 9/631 non-CF strains $(<1 \%)$. The SNPs are in high linkage disequilibrium, with an $r^{2}$ of 0.838; only one strain containing D12S did not also have the S24A mutation, while S24A was found 9 times without D12S. As with the mutations in $p q q F$, I cannot determine if one or both of the mutations are causal as they are in high linkage disequilibrium.

nar $G$ encodes for a respiratory nitrate reductase alpha chain. It is responsible for the anaerobic respiration of PA [149, 150, 151]. A144V is not present in many CF or non-CF strains. It was only found in 86/947 CF isolates (9.1\%), and 13/639 non-CF (2\%).

CysH is a 3'-phosphoadenosine-5'-phosphosulfate reductase identified as being involved in biofilm formation [152]. The mutation L2P is present in almost 50\% of CF strains (456/947) but only $6 \%$ of non-CF strains $(39 / 639)$.

IciA is an inhibitor for chromosome replication initiation that has been studied in E. coli. If it is highly expressed, there is a longer lag time when bacteria are introduced to a new media [153]. It has not been linked to $\mathrm{CF}$ adaptation or virulence at this time. The M1L mutation was present in 
$50 \%$ of CF strains (467/947), but only $5 \%$ of non-CF strains $(33 / 639)$.

\section{3. treeWAS}

TreeWAS gave less corroborated results for the SNP dataset. Few of the most important genes identified were found with other methods or were recognizable in literature. 193 SNPs were found in 44 genes in the simultaneous analysis, most of which were hypothetical. Most of the named genes found in Table 10 were described in Section 5.2. Given the spikes in the treeWAS Manhattan plot (Figure 21), several of the SNPs identified are in LD, meaning that not all of them may actually contribute to adapting to a CF-lung environment, but rather that they are inherited together due to their proximity on the chromosome. The top two mutations in $p q q F$ are a good example of this. The spike just after position 30,000 in Figure 21 represents a number of SNPs found in just one gene, encoding hypothetical protein PA3290.

Table 10: Top SNPs and indels found in treeWAS combined association analysis.

\begin{tabular}{llll}
\hline Rank & Gene & Mutation & Association Score \\
\hline 1 & $p q q F$ & H3R & 51 \\
2 & $p q q F$ & H12R & 50 \\
3 & tle1 & D168E & 45 \\
4 & $i c i A$ & M1L & 44 \\
5 & PA5270 & S5A & 43 \\
6,7 & PA1355 & F33S, C37G & 42 \\
\hline
\end{tabular}

tle1 was not identified in the PLINK analysis. It is a type VI secretion phospholipase effector that targets and hydrolyzes membrane phospholipids. It is part of the T6SS, which has antibacterial properties [154]. In K. pneumoniae, T6SS has been induced by sublethal antibiotic concentrations (tested with beta-lactams and apramycin), and activation of tle1 may make isolates more lethal to other bacteria present [155].

\subsection{Random forest}

The random forest model had 92.5\% accuracy and identified 296 SNPs needed to predict the phenotype of an isolate. Many of the genes identified were hypothetical or found through other analyses.

Several of the mutations found with a random forest were identified through PLINK and treeWAS (Table 11). The functions of $p q q F$ and $c y s H$ were previously discussed in the PLINK section. 


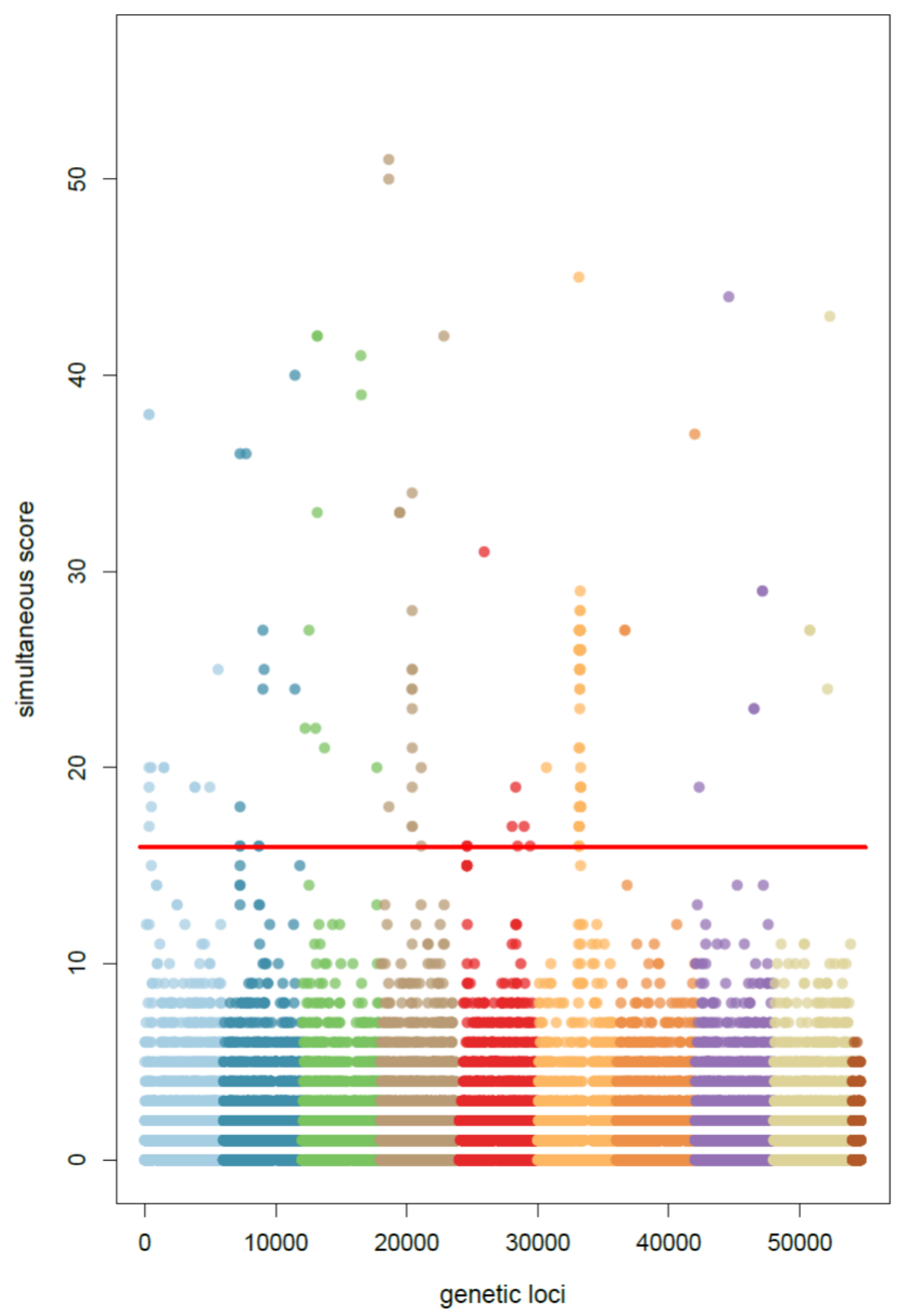

Figure 21: Manhattan plot of SNP scores found from treeWAS association simultaneous analysis. 
K92R in pqqF, a mutation not identified in PLINK, was present in over 90\% of CF strains (865/947), and almost $95 \%$ of non-CF strains $(607 / 639)$, but it is unclear how this mutation affects the function of the protein. $w b p M$ is involved in the O side chain synthesis of the LPS and makes the O-antigen more temperature dependent [156]. Mutations to this gene can change the stability of the O-antigen which can adapt and avoid immune responses from the host. If the gene is knocked out, there is no O side chain produced in the LPS [157]. The V113P mutation was present in only $4.8 \%$ of CF strains (46/947), and over 40\% of non-CF strains (278/639). YecS is an L-cystein transporter of the $\mathrm{ABC}$ system that is involved in host adaptation. It has not been well characterized, but studies have linked it to being involved with CF strains of PA [158, 23]. In this dataset, an insertion at position 165 was only found in CF strains (252/947 CF strains have the mutation, but only 1 non-CF strain has been found with the mutation).

Table 11: Top SNPs and indels that contribute to the random forest model.

\begin{tabular}{llll}
\hline Rank & Gene & Mutation & Importance \\
\hline 1 & $p q q F$ & H12R & 0.14 \\
2 & $p q q F$ & H3R & 0.098 \\
4 & $w b p M$ & V113P & 0.049 \\
6 & $p q q F$ & K92R & 0.039 \\
8 & $i c i A$ & M1L & 0.012 \\
9 & cysH & L2P & 0.012 \\
15 & yecS & 164 insertion & 0.008 \\
\hline
\end{tabular}

\subsection{Support vector machine}

SVM had $89.9 \%$ accuracy with the core SNP dataset. Several of the mutations identified were also recovered in PLINK, treeWAS or the random forest approach (Table 12).

Table 12: Top SNPs and indels found in support vector machine model.

\begin{tabular}{llll}
\hline Rank & Gene & Mutation & Absolute hyperplane coefficient \\
\hline $1-4$ & dipeptidase & 21 insertion, G22A, G22V, 22 insertion & 0.5 \\
5 & pirA & A370T & 0.339 \\
6 & mexK & E765A & 0.337 \\
7 & mexK & K694R & 0.291 \\
8,9 & pirA & A228V, V128A & 0.281 \\
10 & mutL & E428D & 0.281 \\
15,16 & wbpM & I7G, K8R & 0.19 \\
18 & gyrA & D87Y & 0.176 \\
20 & yecS & 164 insertion & 0.151 \\
\hline
\end{tabular}


PirA is necessary for the action of siderophore antimicrobial drugs, such as cefiderocol, a catechol conjugate, and MC-1, a monocarbam. Without this receptor, siderophores lose their mechanism of action [116, 159, 160]. The A370T pirA mutation is mostly present in the PA strains studied (913/947 CF, 624/639 non-CF). The other two pirA mutations in Table 12 are absent in most strains (A228V is present in 23/947 CF and 6/639 non-Cf strains, V128A is present in 29/947 CF, 9/631 non-CF). mexK can affect antimicrobial resistance as well, as it encodes for another efflux pump as described in earlier sections. The E765A mutation is present in 65/947 CF strains, and only 18/639 non-CF strains. The K694R mutation has a higher prevalence, found in 30\% of CF strains (283/947) and $44 \%$ of non-CF strains (282/639).

mutL was discussed in the gene presence/absence dataset, (Section 4), as is was missing more often in CF strains than non-CF strains and identified as being important to the CF phenotype. It is involved in DNA repair and loss-of-function can create hypermutator strains. It was present in enough genomes to still constitute a core gene. The mutation E428D is present in few strains: 12/947 CF and 28/639 non-CF.

gyrA encodes the gyrase subunit A protein. The D87Y mutation has been characterized in other studies to contribute to resistance to antimicrobial drugs, namely fluoroquinolones [32, 33, 161]. The D87Y mutation was present in 29/947 CF strains, and only 9/639 non-CF strains, as might be expected in $\mathrm{CF}$ patients treated with quinolones.

\subsection{Fisher's exact test}

As with the Fisher's exact test done using the gene dataset, thousands of genes had $p$ values less than $1 \times 10^{-20}$, which is significantly smaller than the Bonferroni cutoff of $1 \times 10^{-8}$. The Manhattan plot for the Fisher's exact test (Figure 22) of this dataset shows a few more peaks than the one from section 4 , however the lack of LD is still evident in the significance of SNPs located close together on the chromosome.

\subsection{Principal component analysis}

Principal component 1 splits the CF strains of PA from non-CF strains the best (Figure 23). PC1 accounts for $19.2 \%$ of the variance, while PC2 only accounts for $9.8 \%$. The model required 9 components to account for $50 \%$ of the variance in the dataset and, aside from component 1 , none 
could split the CF from the non-CF strains (Figure 24).

None of the top genes that contribute to PC1 are identified in literature for a CF or AMR phenotype, nor were they found using other analysis methods, aside from perhaps secF. SecF is a membrane protein that may be up-regulated in biofilms [162]. There were three secF mutations identified in PC1, A109T, D219S and V221I. These mutations were all present in 169/947 CF strains, and between 239 and 244 of 639 non-CF strains. 


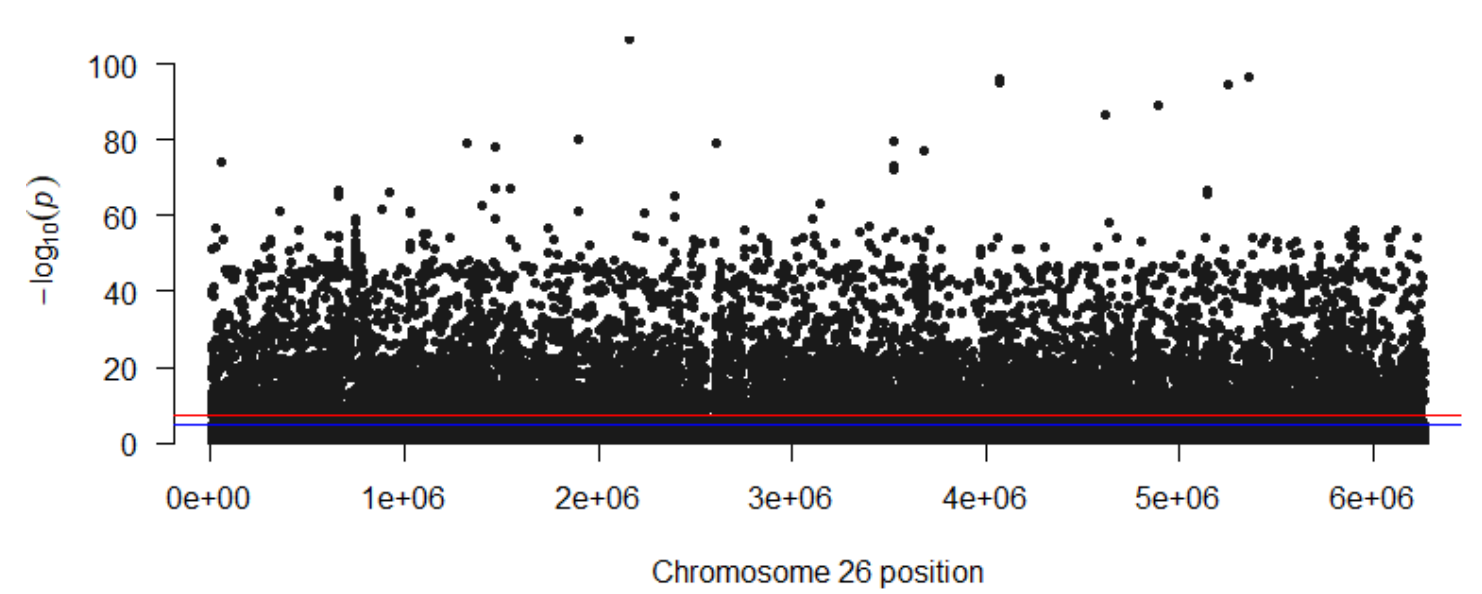

Figure 22: Manhattan plot of $p$ values from a Fisher's exact test for each SNP found in a core gene. The red line is the genome-wide significance line, at $\log \left(1 \times 10^{-8}\right)$. The blue line is the suggestive significance line, at $\log \left(1 \times 10^{-5}\right)$.
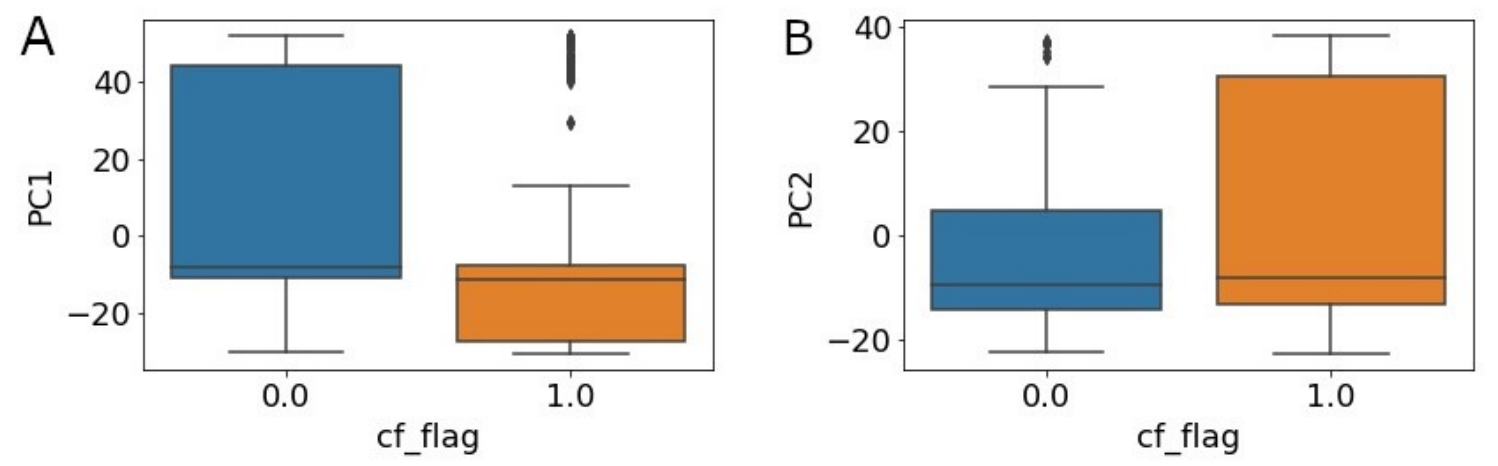

Figure 23: Boxplot of mean principal component values of mutations for cystic fibrosis and noncystic fibrosis strains. CF_flag of 1 is a cystic fibrosis strain, $C_{-}$_flag of 0 is a non-cystic fibrosis strain. A) Principal component 2 mean values, B) principal component 3 mean values. 


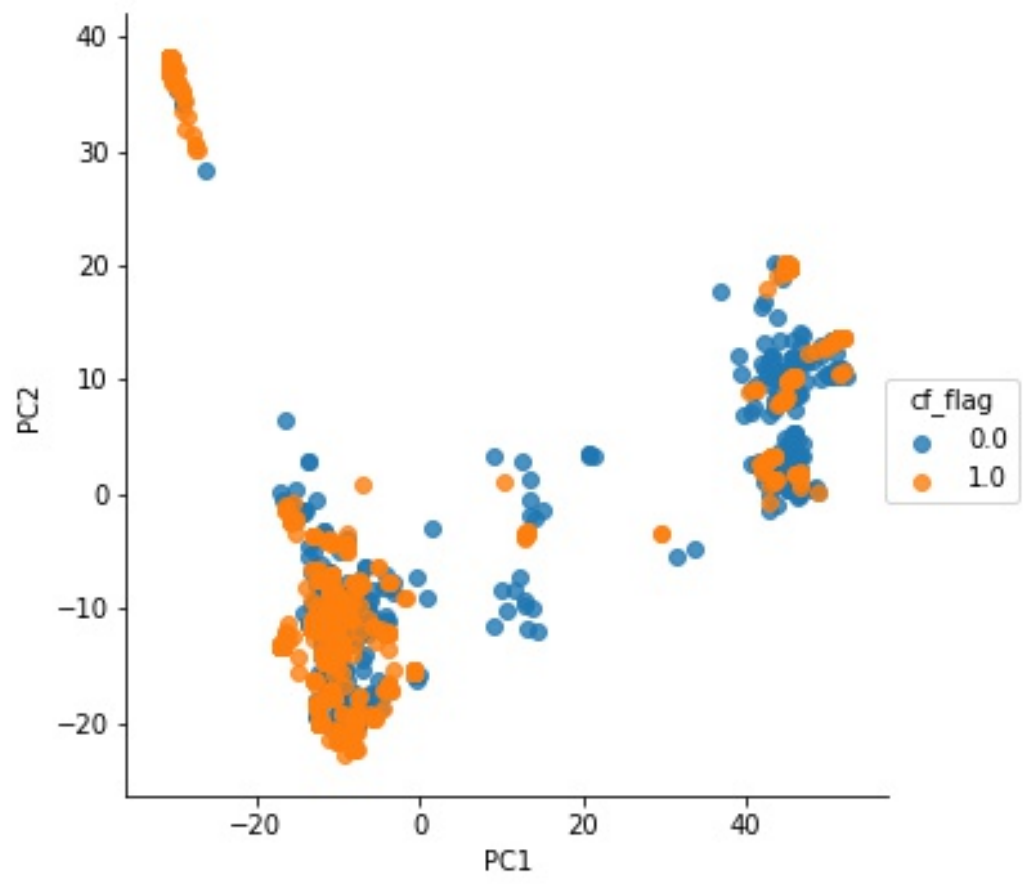

Figure 24: Plot of principal component 2 values against principal component 1 for mutations of cystic fibrosis and non-cystic fibrosis P. aeruginosa strains. 


\subsection{Conclusion of core SNP dataset}

I examined associations for the mutation dataset, consisting of SNPs or indels (insertion/deletions) within 4653 core genes. Some genes were excluded from the original "core" genome due to poor alignment. This could be corrected for by removing BLAST matches that have a lower threshold in common with the reference, but risks removing genes that are highly similar but short in length. One remedy for this would be using a program such as SuperCRUNCH [163] to find better matches for sequences.

A number of SNPs were found in common between the various methods. Many of these variants were in hypothetical proteins (Figure 25). Two mutations in $p q q F, H 3 R$ and H12R, were identified with all four methods, although they were less significant in the SVM model compared to the other three analyses (Figure 26). Mutations in iciA and $c y s H$ were also recovered in all four analyses.

The SVM identified the largest number of mutations within genes that have known functions. At least one of these mutations is already in literature as contributing to quinolone resistance (D87Y in $g y r A$ ). Many genes identified in other methods were also recovered in the SVM analysis (Figure 26, although with a lower ranking in SVM compared to the other methods. 


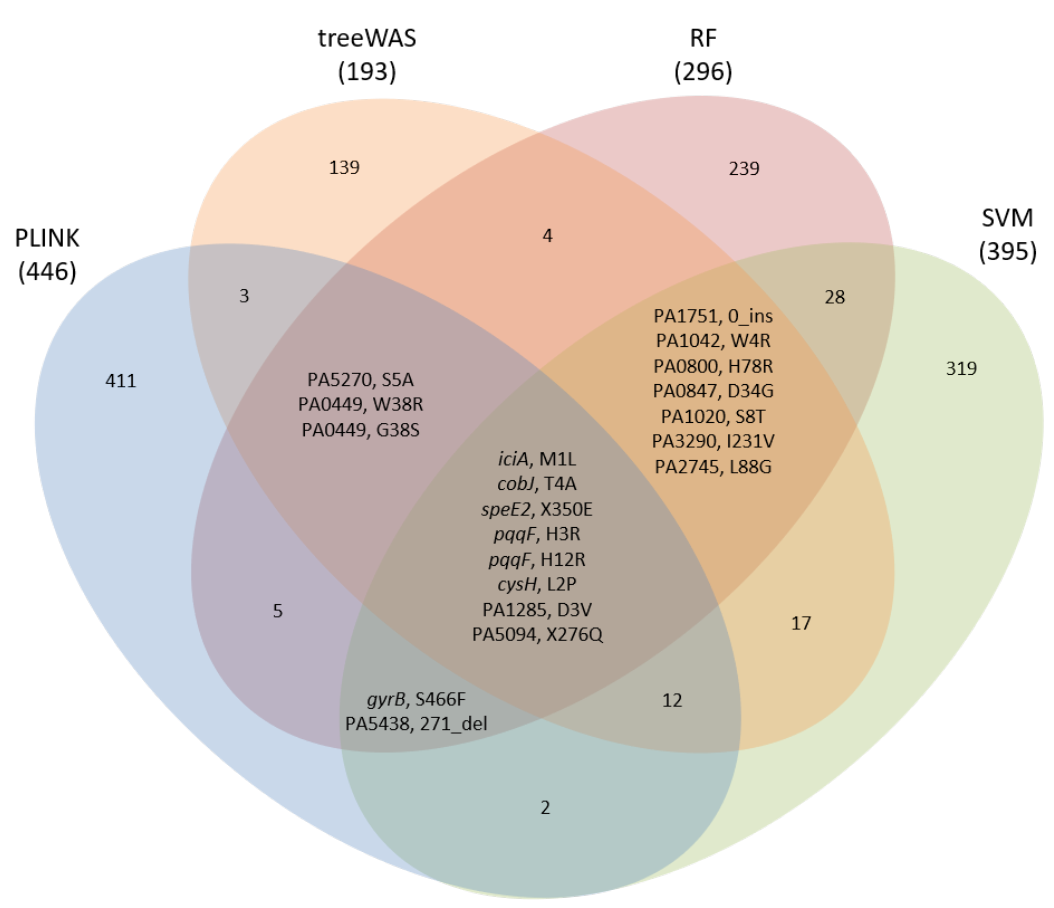

Figure 25: Venn diagram of results from PLINK, treeWAS, random forest and support vector machine. SNPs identified in at least 3 of the 4 methods are reported, if there were less than 10. 


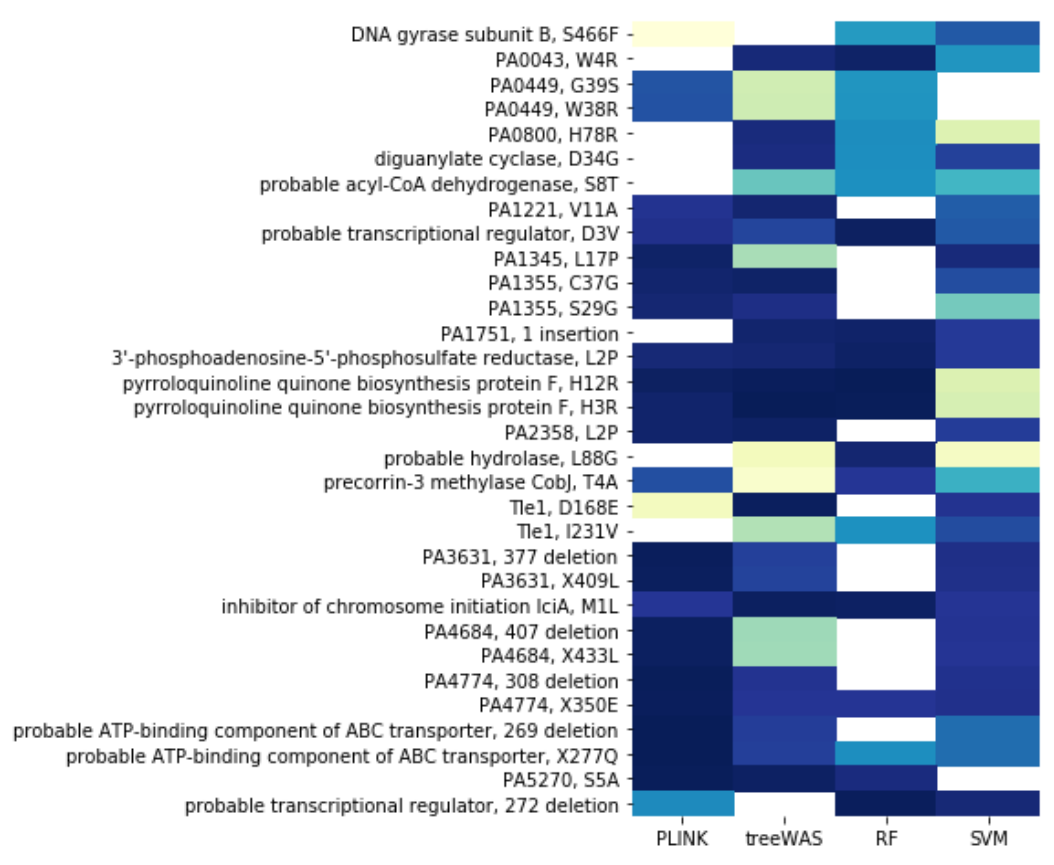

Figure 26: Heatmap of SNPs found in the core SNPs dataset with various methods. Darker blue is a higher ranking (lower $p$ value or higher association score/ importance/ hyperplane coefficient) variant. Yellow is a lower ranking variant. White is not identified as contributing to the phenotype. 


\section{GENE AND MUTATION DATASET RESULTS AND DISCUSSION}

This dataset was created by combining the gene and mutation datasets (Sections 4 and 5 ).

\subsection{PLINK}

The QQ plot from the PLINK analysis of the full dataset has a similar structure to those found with the separated gene and SNP sets (Figure 27), suggesting that some non-causal variants are confounding the PLINK results. The Manhattan plot (Figure 28) shows that many genes and SNPs were identified as contributing to a CF phenotype in the PLINK analysis. There are too many variants to be researched, and it is very likely that many of the variants identified are not truly causal. The PLINK results from the combined datasets should be taken with caution.

Most of the genes and SNPs identified by PLINK in the combined analysis (Table 13) were not discussed earlier. The G5 domain containing protein is a hypothetical gene found in PA7. It is absent in all but 2 CF strains, but is present in 37/639 non-CF strains. G5 domains in proteins have been identified as $\mathrm{N}$-acetylglucosamine binding sites, and could be related to biofilm formation, at least in Streptococcus [164]. Another hypothetical protein identified was a probable helicase. This gene has not been experimentally identified or studied, however some RNA helicases have been shown to impact biofilm formation [165, 166]. An A8S mutation in this gene was present in 15/947 CF strains, but only 1/639 non-CF strains. The E214D mutation was found almost evenly in CF and non-CF strains, appearing in 15 and 12 isolates respectively.

Table 13: Top genes and SNPs from from PLINK stratified association analysis

\begin{tabular}{llll}
\hline Rank & Gene & Mutation & p value \\
\hline 1 & G5 domain containing protein & & $2.68 \times 10^{-60}$ \\
2,3 & hypothetical helicase & A8S, E214D & $1.59 \times 10^{-49}$ \\
5 & fdhE & A235T & $1.79 \times 10^{-45}$ \\
8 & glpD & R274H & $8.97 \times 10^{-44}$ \\
10,11 & met & V76A, E659Q & $3.90 \times 10^{-40}$ \\
12,13 & pvdE & E327S, S394E & $5.12 \times 10^{-40}$ \\
14 & tonB dependent receptor & L14P & $3.37 \times 10^{-39}$ \\
\hline
\end{tabular}

Two mutations were identified in two proteins involved in metabolism. FdhE is involved with ferric iron binding and has been studied with regards to energy metabolism in bacteria [167], but has not been linked to CF-related adaptations. An A235T mutation was found in 28/947 CF strains and 


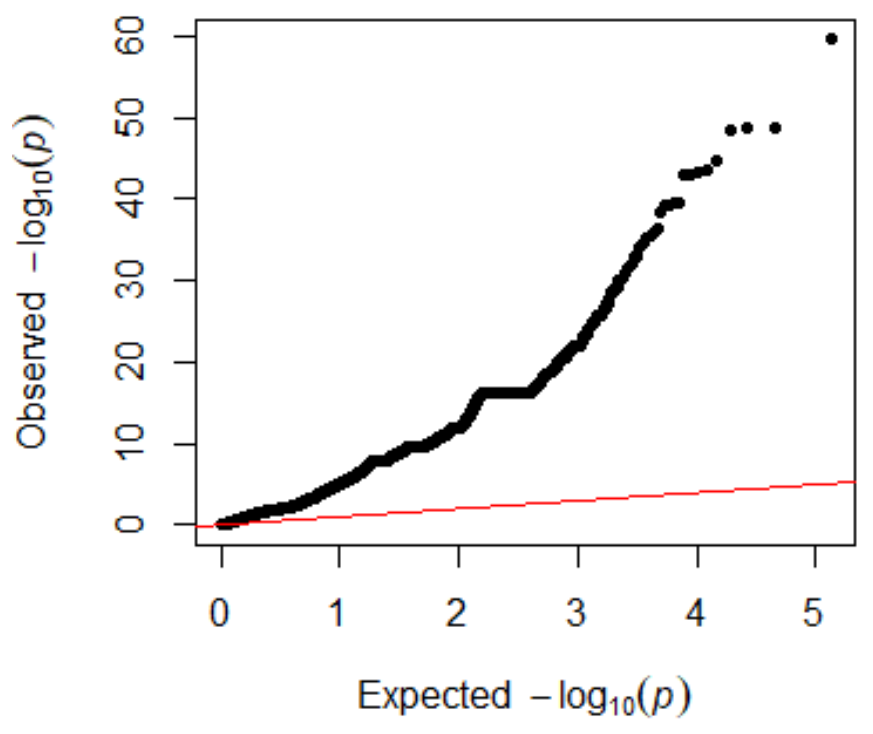

Figure 27: QQ plot of calculated $\log p$ values in PLINK of the gene and SNP datasets against expected $p$ values. The red line is when the observed $p$ values equal the expected $p$ values $(\mathrm{x}=\mathrm{y})$

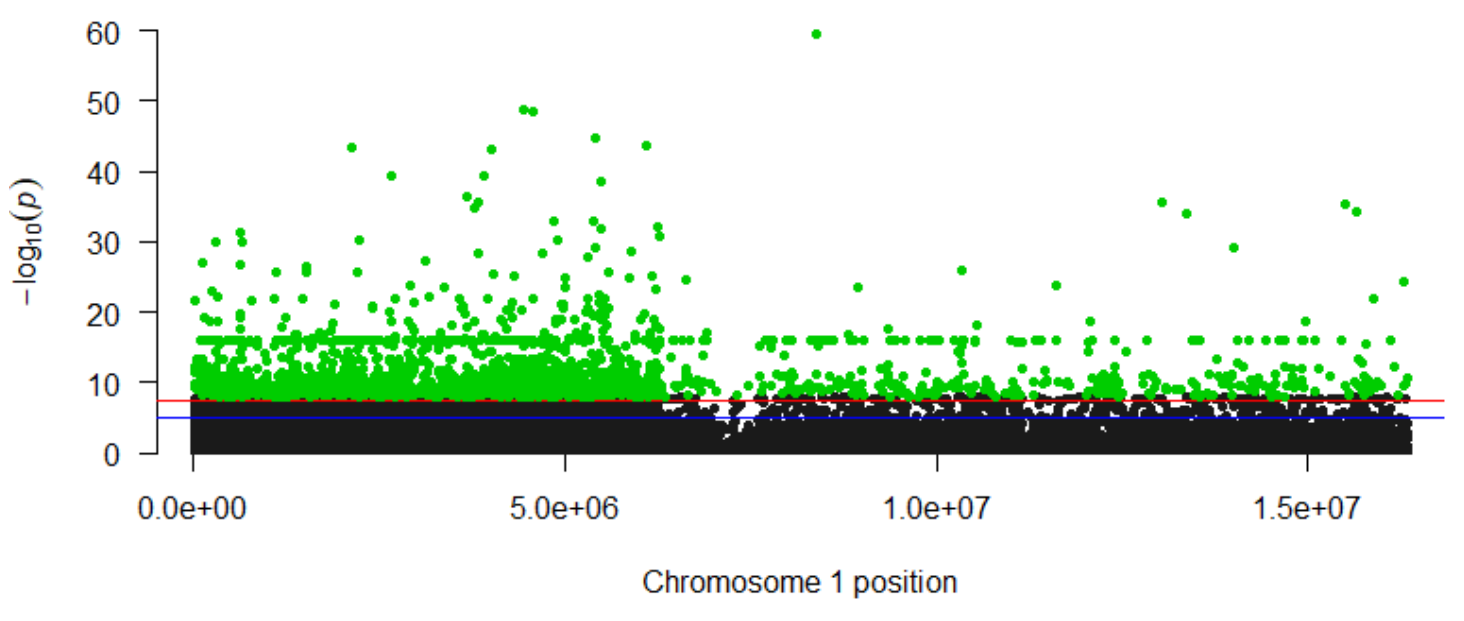

Figure 28: Manhattan plot of $p$ values in the PLINK analysis of the gene and SNP dataset. Points above the red line are significant, and coloured in green. The red line is the genome-wide significance line, at $\log \left(1 \times 10^{-8}\right)$. The blue line is the suggestive significance line, at $\log \left(1 \times 10^{-5}\right)$. 
47/639 non-CF PA strains. glpD encodes for a protein involved in glycerol metabolism. Mutations and changes its activity can affect the metabolism of amino acids, induce a stress response with $\operatorname{rpoS}$ [168], and lower alignate production making isolates much more virulent [169]. The R274H mutation was in only a single CF strain of PA and 38/639 non-CF strains.

MetG, or methionyl-tRNA ligase, is required to initiate and elongate protein synthesis. Changes in the C-terminus of the gene metG has been shown to cause increased virulence in E. coli [170], however the effects have not been studied in PA. The V76A mutation was only found in CF strains of PA (16/947, or 1.7\%), while the E659Q mutation was found in both CF and non-CF isolates (215/947, or $22.7 \%$, CF strains and 159/639, 24.9\%, non-CF strains).

pod genes are involved in the synthesis of pyoverdine, a siderophore. These genes are involved in iron acquisition [171], which is a virulence adaption of PA in a CF lung. Both the E327S and S394E mutations were in over half of the CF strains $(537 / 947,56.7 \%)$, but less than half of the non-CF strains (278/639 and 280/639 isolates, respectively, 43.5-43.8\%). Most studies of the pyoverdine pathway and feedback loops are of podS, not pvdE, however this pathway does differ in CF environments compared non-CF ones [123, 22]. $p v d L$ was found in a longitudinal PA study to have a high number of SNPs during chronic lung infection [17].

The importance of TonB receptors was discussed in earlier sections (Section 4.2), however the L14P mutation was not found in the SNP-only dataset analyses. This mutation was uncommon in the dataset, but found more often in non-CF strains $(<1 \%$ of CF isolates, $7 / 947$, but $3.4 \%$ of CF strains, 22/639).

\section{2. treeWAS}

Many of the genes identified in the treeWAS analysis of the combined gene and SNP dataset (Table 14. Figure 29) were already identified in previous PLINK and treeWAS sections (Sections 4.2, $4.3,5.2$ and 5.3. The one gene not previously discussed was mas $A$, which encodes for enolase-phosphatase E-1, and was the only gene listed in Table 14 that was not previously discussed. The gene is more often found in non-CF strains of PA (608/639, but only 483/947 non-CF strains). A link between mas $A$ and adaptations to a CF lung environment has not been studied as of yet. 


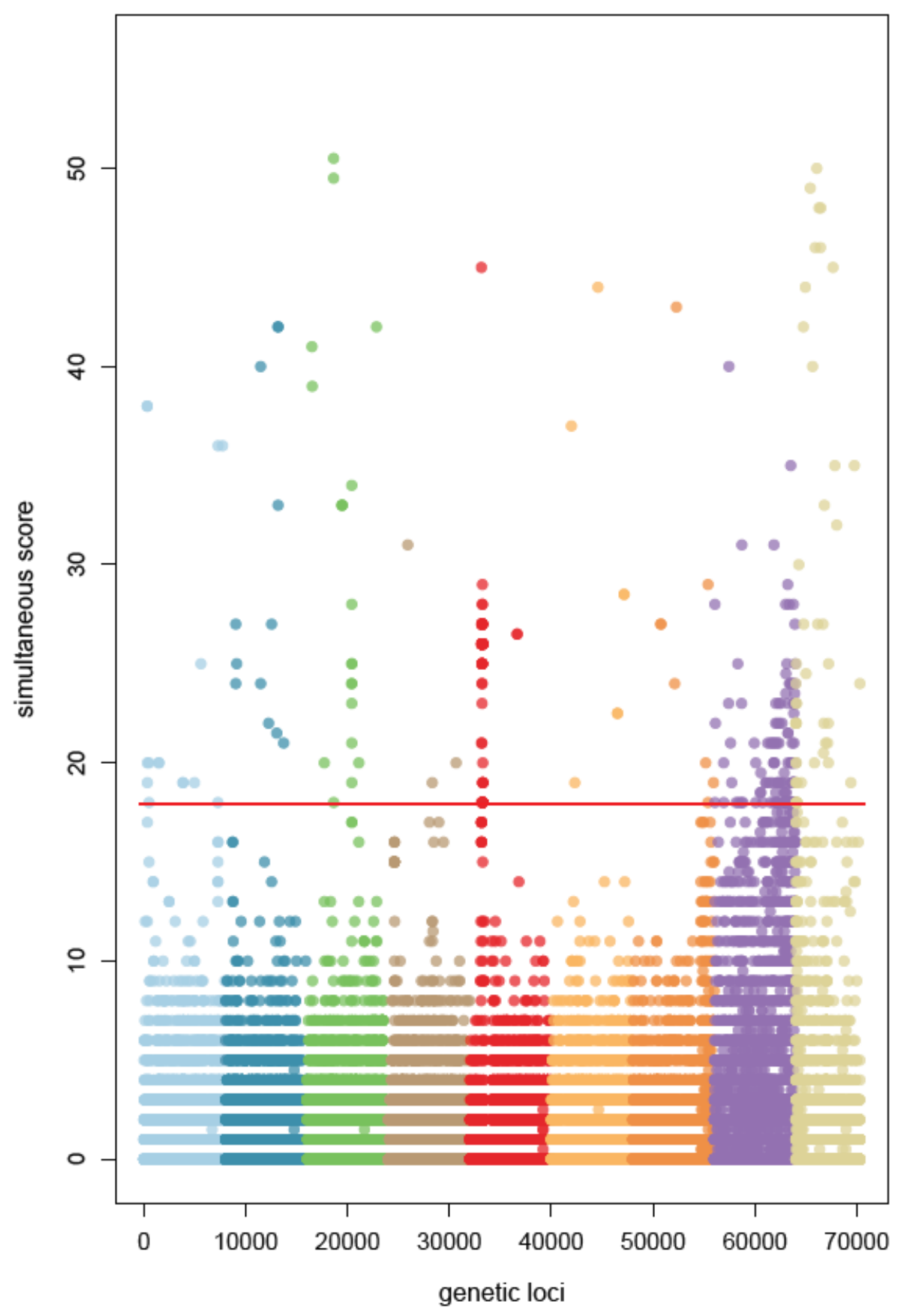

Figure 29: Manhattan plot of gene and SNP scores found from treeWAS association simultaneous analysis. 
Table 14: Top genes and SNPs found in treeWAS simultaneous association analysis.

\begin{tabular}{llll}
\hline Rank & Gene & Mutation & Association Score \\
\hline 1 & pqqF & H3R & 50.5 \\
2 & protease & & -50 \\
3 & pqqF & H12R & 49.5 \\
4 & hxcY & & -49 \\
7 & napA & & -46 \\
8 & mas $A$ & & -46 \\
9 & tle1 & D468E & 45 \\
11 & iciA & M1L & 44 \\
12 & tonB2 & & -44 \\
17 & recF & & -42 \\
22 & cysH & L2P & 39 \\
\hline
\end{tabular}

\subsection{Random forest}

As with the treeWAS combined analysis, many of the genes and SNPs in the random forest model (Table 15) were found and described in earlier sections (Sections 4.2, 4.4, 5.2 and 5.4).

Mutations within $m u c D$ and $\arg E$ were not mentioned in earlier sections. MucD is a protein that regulates the alginate pathway by activating the degradation of MucA [172, 173]. Alginate production affects biofilm formation and virulence of bacterial strains. $m u c D$ does not play as large a role in mediating alginate as $m u c A$, however finding a mutation in $m u c D$ that contributes to the adaptation of PA in a CF lung is not surprising. The V440I mutation was in 262/947 CF isolates (27.7\%), 202/639 non-CF (31.6\%). Acetylornithine deacetylase, encoded by $\arg E$, is involved in amino acid metabolism [174] and arginine biosynthesis [175]. Arginine synthesis has been predicted to be involved in biofilm formation [176], but this has not been experimentally confirmed.

Table 15: Top genes and SNPs that contribute to the random forest model.

\begin{tabular}{llll}
\hline Rank & Gene & & Mutation Importance \\
\hline 1 & PA2074 & & 0.262 \\
2 & mucA & & 0.073 \\
3 & pqqF & H3R & 0.051 \\
4 & tonB2 & & 0.038 \\
9 & pqqF & H12R & 0.010 \\
19 & wbpM & V113P & 0.005 \\
23 & mucD & V441I & 0.005 \\
24 & argE & R221Q & 0.005 \\
\hline
\end{tabular}




\subsection{Support vector machine}

Several of the genes and SNPs in Table 16 were discussed previously (Sections 4.2, 5.4, and 5.5. The second and third genes identified with the SVM approach were probable dioxygenases, with no experimentally confirmed function. One is present in 448 of $947 \mathrm{CF}$ and 309 of 639 non-CF strains, while the other is found in 497 of 947 CF and 319 of 639 non-CF isolates. Though the exact function of these genes is currently undescribed, various dioxygenases have been linked to virulence and prevalence of chronic PA infections [177]. Another poorly described protein identified was an isomerase, 2,3-dihydro-3-hydroxyanthranilate isomerase. This protein has not been experimentally studied, but is similar to a protein produced by $p h z F$, which is part of the phenazine biosynthesis pathway. Another $p h z$ gene was previously discussed, $p h z E$, in section 4.5 . This isomerase was present in only 80 of 947 CF isolates, and 82 of 639 non-CF strains.

mexA encodes an efflux pump component, similar to mexB, mexZ and mexK previously discussed (Section 4.2. mexA was absent more often in CF isolates when compared with non-CF (absent in 53/947 CF, 5.6\%, and 10/639 non-CF, 1.6\%).

exaC produces an aldehyde dehydrogenase $\mathrm{ExaC}$, which is part of energy and metabolism pathways [178]. Its role in growth in different environments is debated [178, 179], and has not been linked to beneficial or detrimental effects in CF lung environments. The gene was missing in more CF strains, present in only $52.7 \%$ of CF strains (499/947), but was present in $73.6 \%$ of non-CF strains $(470 / 639)$.

Table 16: Top genes and SNPs found in support vector machine model

\begin{tabular}{llll}
\hline Rank & Gene & Mutation & Absolute hyperplane coefficient \\
\hline 1 & mucA & & 0.0432 \\
2,3 & dioxygenase & & $0.0337,0.0320$ \\
4 & isomerase & 0.0315 \\
5 & tonB2 & & 0.0300 \\
7 & mexA & 0.0281 \\
8 & yecS & \multirow{2}{*}{ in insertion } & 0.0279 \\
9 & oprB & & 0.0277 \\
10 & exaC & & 0.0262 \\
16 & pirA & A370T & 0.0244 \\
19 & mexZ & & 0.0235 \\
\hline
\end{tabular}




\subsection{Conclusion}

With the combined gene and SNP datasets, very few genes were found in common between the different analysis methods (Figure 30). No variants were in common between all four methods. Several variants identified in at least three of the methods were found in earlier analyses, such as tonB2, mexZ, recF, and the two mutations in $p q q F$.

PLINK did not have a lot of variants in common with the other methods, and identified over 2000 genes and SNPs that contributed to the CF-phenotype of PA. treeWAS, RF and SVM identified several variants in common, and ranked many of the top ones similarly (Figure 31). Between treeWAS, RF and SVM, none of the methods outperformed the others in terms of identifying genes/SNPs from literature. 


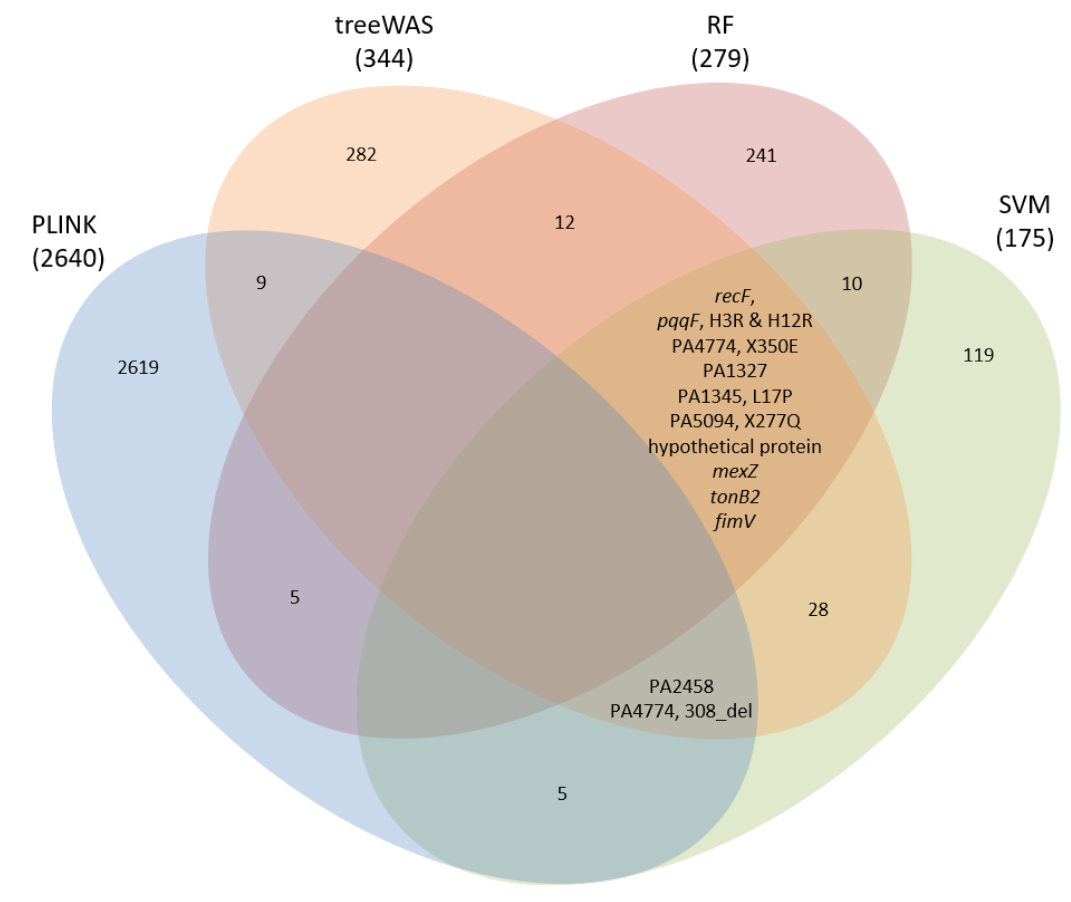

Figure 30: Venn diagram of results from PLINK, treeWAS, random forest and support vector machine. Variants identified in at least 3 of the 4 methods are reported.

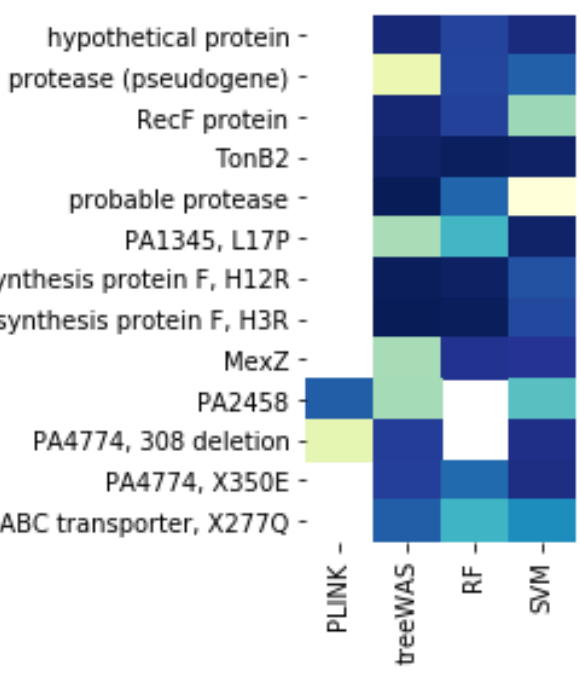

Figure 31: Heatmap of variants found in the combined gene and SNP datasets with various methods. Darker blue is a higher ranking (lower $p$ value or higher association score/ importance/ hyperplane coefficient) variant. Yellow is a lower ranking variant. White is not identified as contributing to the phenotype. 


\section{Select Gene AND mutation DAtASET RESUlts AND Discussion}

This dataset is comprised of the genes and mutations with an importance greater than 0 identified in the random forest model of the gene and mutation datasets (Sections 4.4 and 5.4). PLINK, treeWAS, RF and SVM were run on this smaller dataset, using the same settings as in previous analyses. Random forests are often utilized for feature selection in datasets with many features [180, 181]. I used the variants identified with the random forest model of previous datasets to select important genes and SNPs to be further analyzed by these various methods, in an attempt to gain more relevant and robust results, ideally corroborated by other analyses either with this dataset or previous ones.

Figure 32 is a heatmap of genes and SNPs identified in three of the four methods (PLINK, treeWAS, RF and SVM) with the reduced dataset. The darker the colour, the higher ranked the gene/SNP was in the analysis (ie. lower $p$ value, higher absolute association score, importance or absolute hyperplane coefficient). This provides a comparison of genes and SNPs identified independently in multiple methods, and how highly the variant was ranked.

Many of the genes and SNPs found with PLINK and treeWAS were previously identified with the larger, original datasets. $m u c A, h x c Y$, and $p q q F$ are examples of these. There was not a lot of overlap between SNPs and genes found with an SVM and the other methods. The random forest model and PLINK had a lot of overlap in their results, and RF ranked genes found with other methods quite highly. The two pqqF SNPs (H3R and H12R, found often in other methods) were ranked highly with all the methods in this dataset.

\subsection{PLINK}

Figure 33 shows the observed and expected $p$ values using PLINK on the reduced dataset. The observed $p$ values are much higher than expected, as found with other datasets. Observed $p$ values may not follow a uniform distibution, as many non-causal variants are excluded in this dataset. The tail, or sharp increase, in observed $p$ values is much less pronounced than in previous PLINK analyses.

The Manhattan plot (Figure 34) of the reduced dataset still does not show spikes of genes/SNPs inherited together. There are parts of the genome that were not important to CF adaptation, as there 


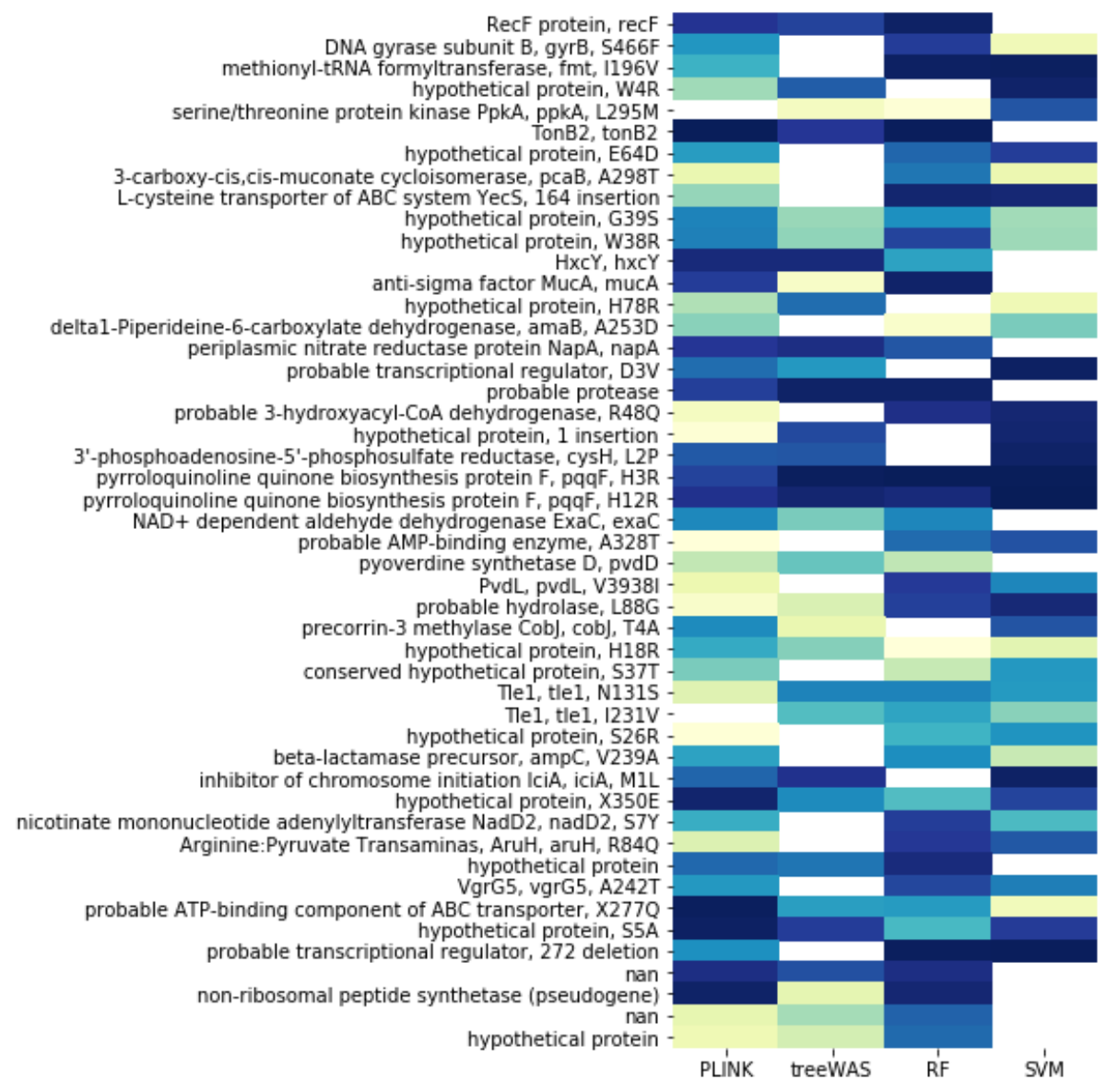

Figure 32: Heatmap of genes and SNPs found in the reduced dataset with various methods. Dark blue is a higher ranking (lower $p$ value or higher association score/ importance/ hyperplane coefficient) variant. Yellow is a lower ranking variant. White is not identified as contributing to the phenotype. 
are much fewer points after position $5.5 \times 10^{6}$.

\section{2. treeWAS}

Figure 35 is a Manhattan plot of the treeWAS simultaneous association scores for the reduced dataset. It is similar to the Manhattan plots of previous treeWAS analysis, with a few spikes of important genes/SNPs. 


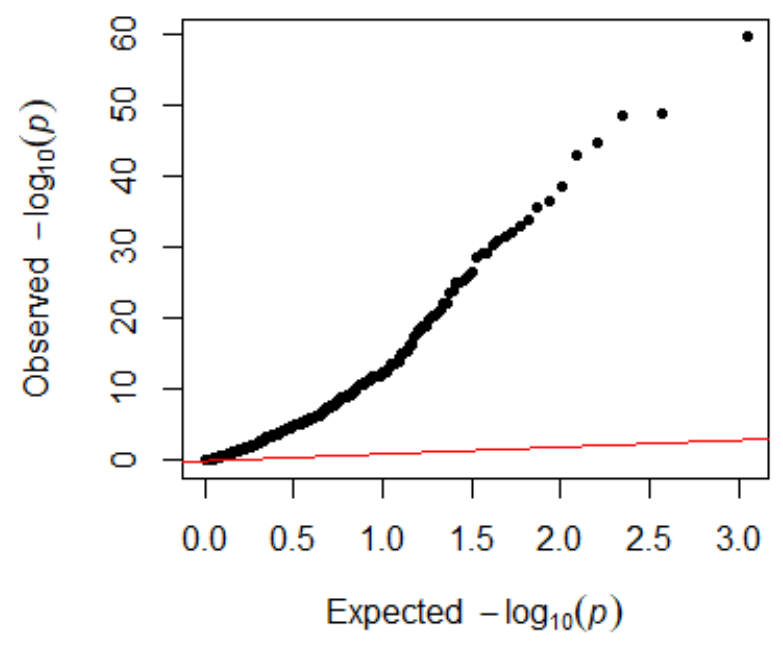

Figure 33: QQ plot of calculated $\log p$ values in PLINK of the reduced gene and SNP datasets against expected $p$ values. The red line is when the observed $p$ values equal the expected $p$ values $(\mathrm{x}=\mathrm{y})$

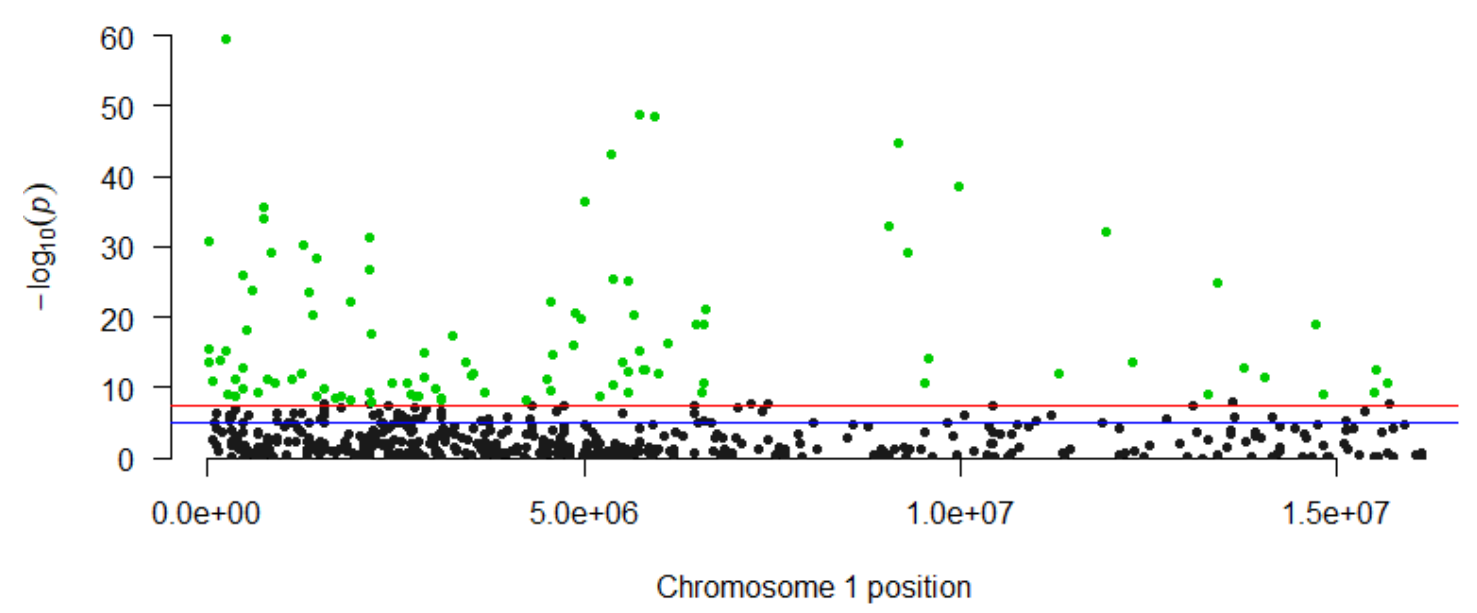

Figure 34: Manhattan plot of $p$ values in the PLINK analysis of the reduced gene and SNP dataset. Points above the red line are significant, and coloured in green. The red line is the genome-wide significance line, at $\log \left(1 \times 10^{-8}\right)$. The blue line is the suggestive significance line, at $\log \left(1 \times 10^{-5}\right)$. 


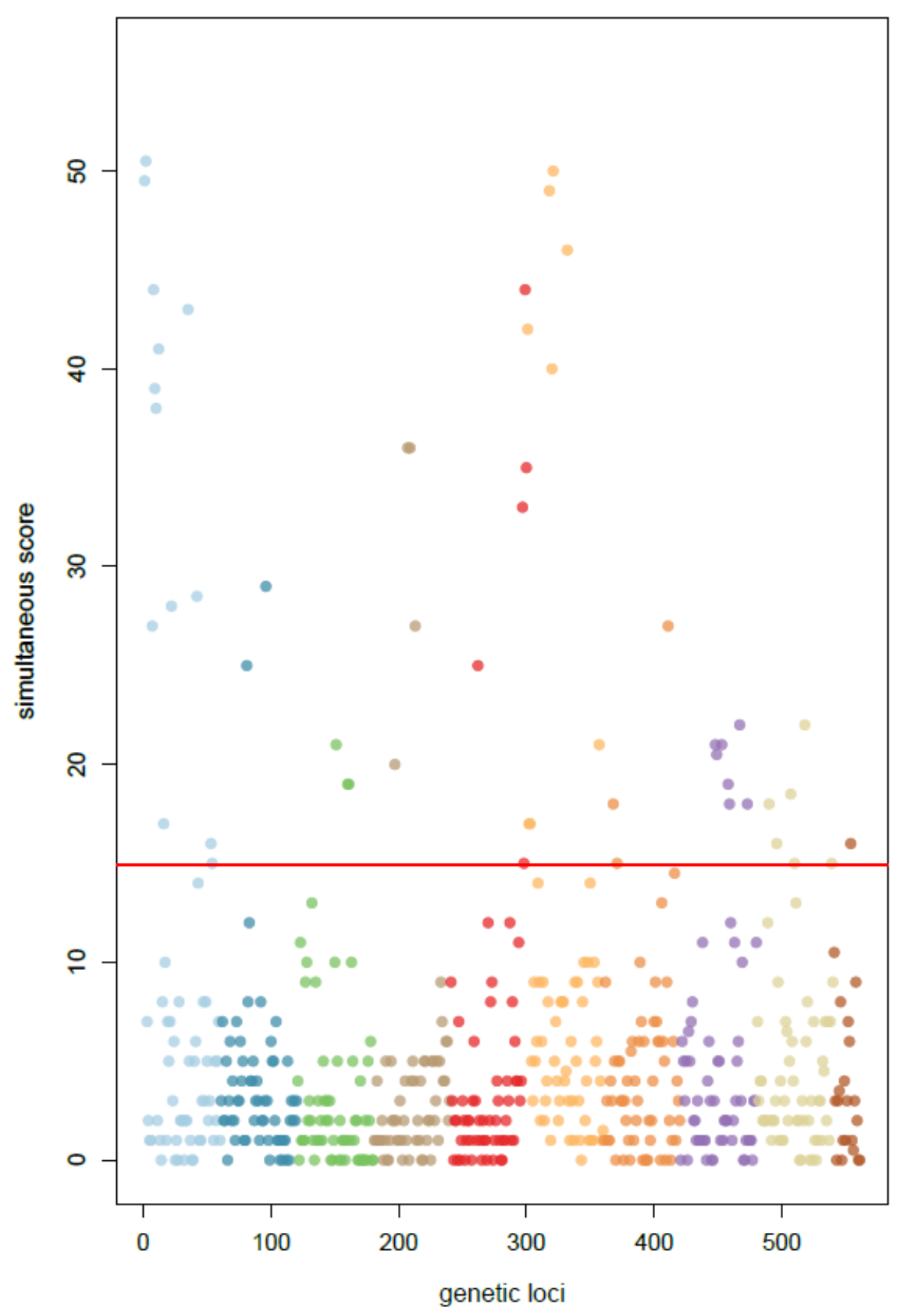

Figure 35: Manhattan plot of reduced gene and SNP scores found from treeWAS association simultaneous analysis. 


\section{RANDOMIZATIONS}

Randomized analyses were run using the gene presence/absence dataset to evaluate the accuracy and results of the various methods. 1000 runs were performed, using the same settings as the original models, but randomly shuffling the phenotype, maintaining the same number of $\mathrm{CF}$ and non-CF strains. The randomized datasets act as an empirical null distribution, allowing us to infer the expected false positive rate for each method. That is, given a random set of data, how many associations does each method find?

\subsection{PLINK}

The PLINK analysis was run using the stratified case/control analysis and the populations as determined previously with fastStructure (Section 4). Figure 36 shows the distribution of $p$ values over the 1000 runs. The blue line with colour under the curve is the distribution of $p$ values from the run with the original dataset. The $p$ values from randomized data trials were evenly distributed, while the original run had an excess of low $p$ values. This suggests that the original dataset had valuable information pertaining to the CF phenotype of PA.

\section{2. treeWAS}

treeWAS was run using the randomized phenotypes and the original phylogeny from ExaML (Section 3.1. Figure 37 shows the distribution of $p$ values in the original and randomized trials. The shaded blue curve is the distribution of $p$ values with the original data. There is no obvious difference between the original $p$ value distribution and the randomized ones, and thus results from treeWAS should be taken with caution. treeWAS creates a null dataset and determines the distribution of $p$ values and associations from the null dataset. It then chooses a cutoff association score from this distribution, and assigns a $p$ value of 0 to any variant in the actual dataset that is

higher than the cutoff. It is unclear why the randomized data does not follow a uniform distribution that is distinct from the true data results. 


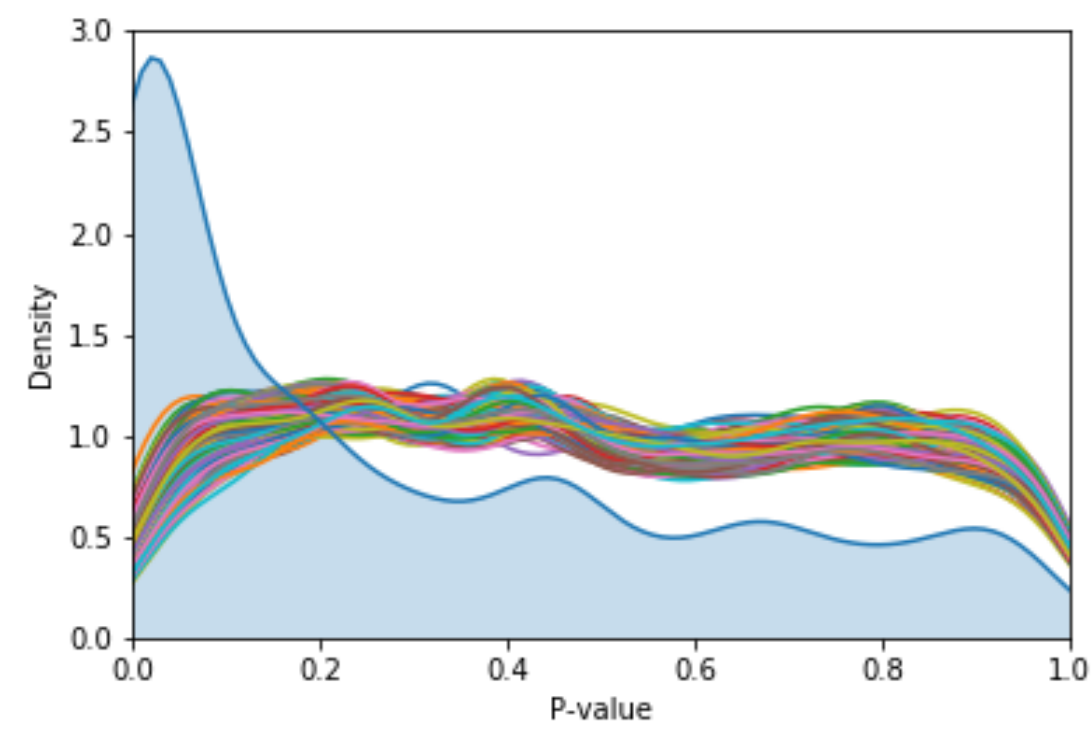

Figure 36: Distribution of p-values from 1000 randomized PLINK model iterations. Blue, shaded line is the distribution of $\mathrm{p}$-values from the original run.

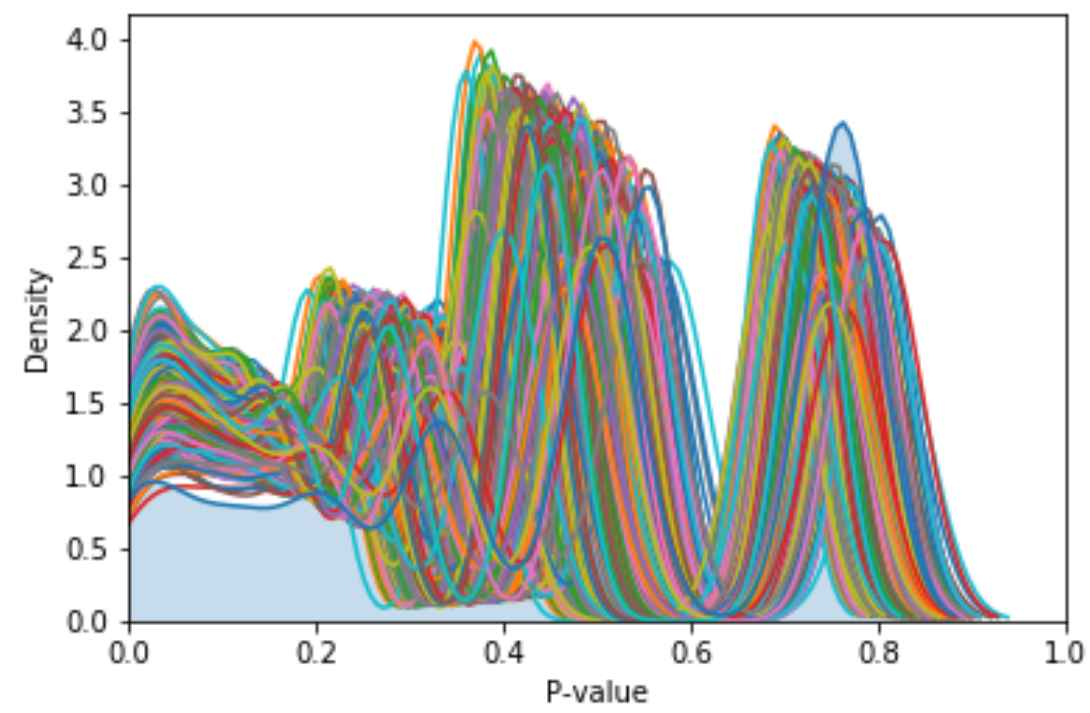

Figure 37: Distribution of p-values from 1000 randomized treeWAS simultaneous association model iterations. Blue, shaded line is the distribution of $\mathrm{p}$-values from the original run. 


\subsection{Random Forest and Support Vector Machine}

With both machine learning methods, accuracy in the randomized datasets dropped from around $90 \%$ to $50 \%$. Randomized RF trials had an accuracy of $52 \% \pm 2.2 \%$ (Figure 38 , while SVM accuracy dropped to $51.8 \% \pm 2.5 \%$ (Figure 39). The RF models needed over 900 genes to explain the variance between $\mathrm{CF}$ and non-CF isolates, compared to 265 genes with the original model. This suggests that both the RF and SVM models were able to find meaningful genes within the dataset and that there were correlations between the phenotype and presence/absence of some genes.

\subsection{Conclusion}

In conclusion, PLINK, RF and SVM were not able to find meaningful differences in CF from non-CF strians when phenotype data was randomized. This is encouraging for the results from these methods, suggesting that true correlations were found between genotype and phenotype. treeWAS found correlations between genes/SNPs and the phenotype even with randomized data. The null hypothesis of having no correlation between genotype and phenotype has a similar distribution of $p$ values as the model with the original dataset. Thus, we cannot reject the hypothesis that there is no correlation between the CF phenotype and some genes/SNPs using treeWAS.

\section{Conclusion}

Machine learning is an artificial intelligence method that has recently been used in human and bacterial GWAS. Both random forests and support vector machines have been used in GWAS with success, however large datasets (hundreds, ideally thousands, of samples) are required to provide meaningful results. Interestingly, these methods may produce less false positives when compared with non-machine learning statistical methods [106]. The use of machine learning in GWAS is expanding, and requires more study into which models are most appropriate for GWAS datasets. For example, one bacterial GWAS used PLINK, then a random forest, to find significant associations [89]. Two others used random forests alone, albeit with small sample sizes (42 and 13 genomes, respectively) [74, 95]. An SVM has been used in one study of 1595 strains of M. tuberculosis, and was successful in finding 33 known and 24 novel genes associated with antibiotic resistance [96].

Here, I used both machine learning and non-machine learning GWAS approaches to identify 


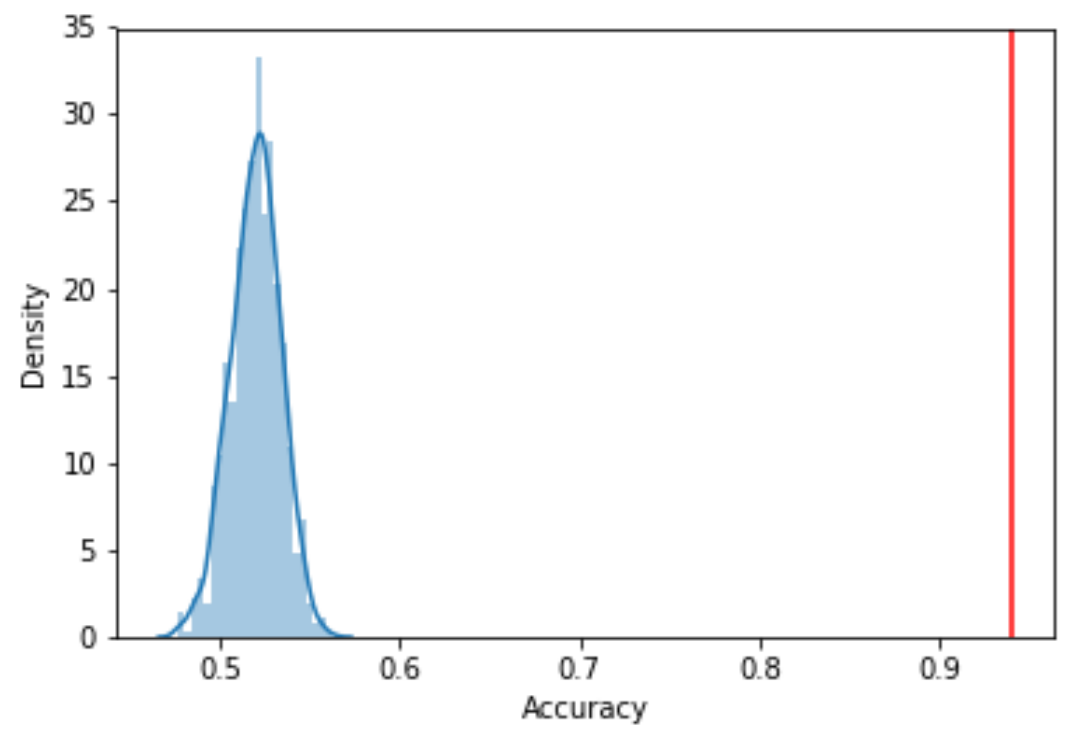

Figure 38: Accuracy of 1000 randomized random forest model iterations. Red line is the accuracy of the model with original data.

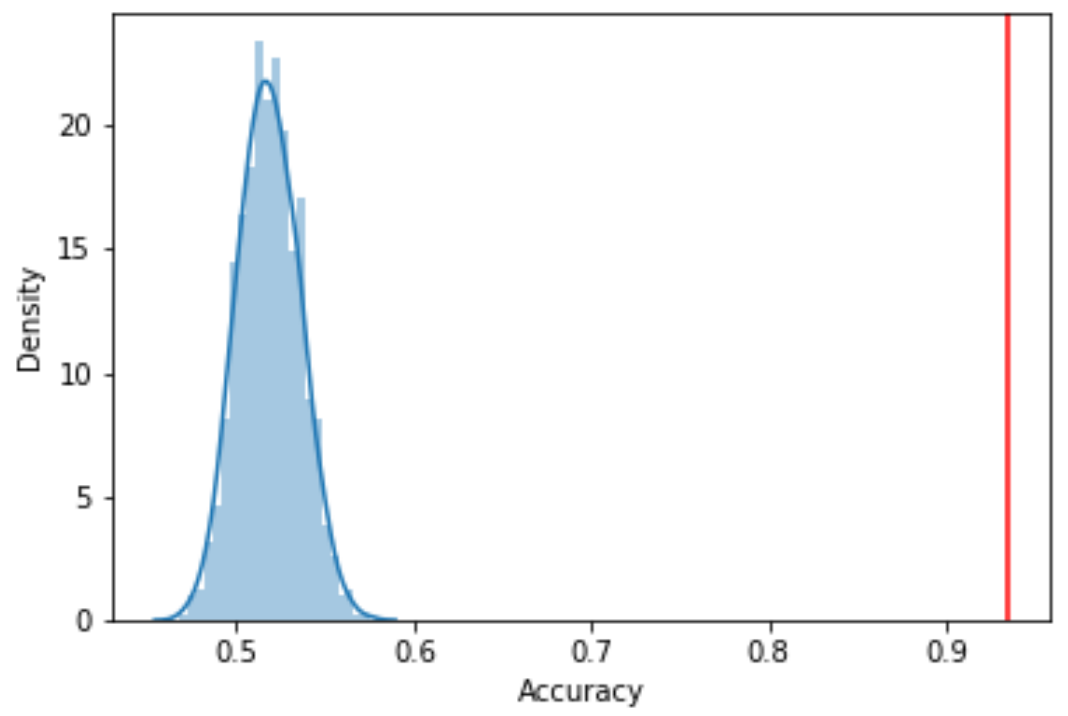

Figure 39: Accuracy of 1000 randomized support vector machine model iterations. Red line is the accuracy of the model with original data. 
CF-pathoadaptive variants in P. aeruginosa. P. aeruginosa is a ubiquitous bacterium that causes chronic lung infections in cystic fibrosis patients. Since these bacteria are directly related to the mortality and morbidity of CF patients, there is a large body of research on the treatment of these infections. PA has been shown to evolve in the lung to become better adapted to this unique environment. There have been several longitudinal studies examining changes in the bacterium over the course of a patient's lifespan and infection [17, 18, 19, 182]. Many of these changes during chronic infection include resistance to antibiotics, changes in expression of virulence factors, increases in mutation rate, formation of biofilms and changes in metabolism [14, 15]. I performed a GWAS to find novel evolutionary changes that PA undergoes in a CF lung, as well as differences between CF and non-CF populations of PA, and compared results between a variety of datasets and methods.

The full GWAS dataset consisted of 15,639 genes in the pan-genome, and 54,662 SNPs or indels in 4652 core genes. In the genome-wide association analyses, the separated gene and SNP datasets (1 and 2, Sections 4 and 5) seemed to perform the best. The fully combined dataset (dataset 3, Section 6) had no variants in common between PLINK, treeWAS, SVM and RF analyses. PLINK went from finding a few hundred variants, to finding a few thousand being significant to a CF-phenotype. TreeWAS stayed most consistent in results when combining datasets (Figure 40. SVM found many variants in common with the combined and separated datasets, however the hyperplane coefficients and ranks differed between the analyses. Random forest was most consistent between datasets 1, 2, and 3, although overall it also reported different variants between the different datasets.

With the gene presence/absence dataset (1, Section 4), a random forest gave the most promising performance, with many high ranking genes found in the literature, and some hypothetical genes for further research. An SVM was most promising with the SNP dataset (2, Section 5), with the fewest highly ranked hypothetical genes, and recovering more genes and SNPs from literature than the other methods.

The most important genes identified in the random forest analysis were involved in DNA repair (recF, mutL), antibiotic resistance $(m e x F)$, alginate production $(m u c A)$, metabolism/biosynthesis (tonB2), and signalling ( $p d t A)$. Several hypothetical proteins, including PA2074 and PA5037, were also identified in the top-ranking genes, and were recovered in other analyses. The importance values in the model decrease sharply after the first few genes. Further research should be concentrated on high ranking hypothetical proteins and other genes with unconfirmed function (such as PA1238, a 

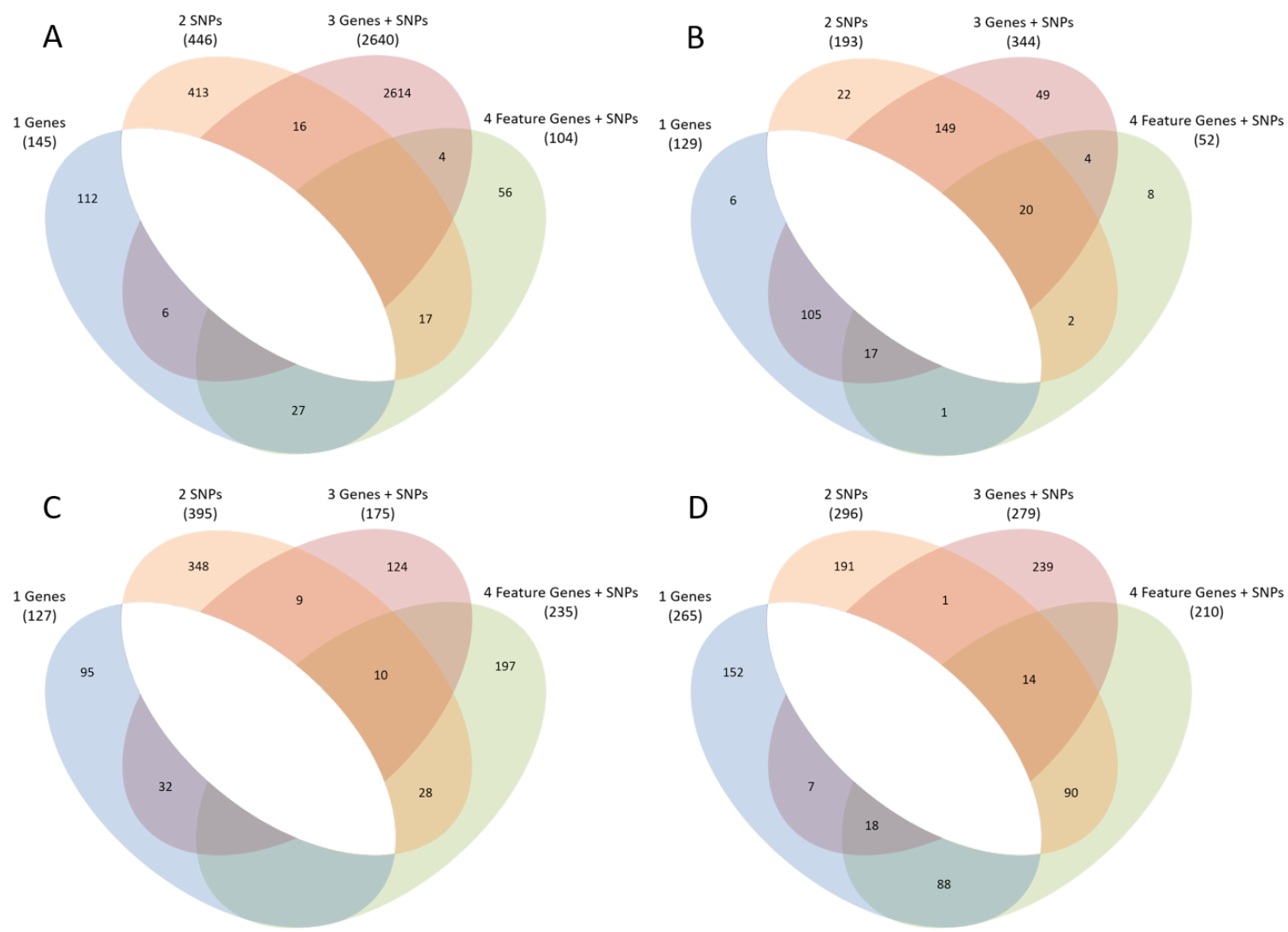

Figure 40: Variants in common between datasets for the various methods. A) PLINK, B) treeWAS, C) random forest, D) support vector machine. Datasets 1 and 2 (gene presence/absence and core SNPs) had no variants in common, as illustrated by blank spaces in the Venn diagrams. 
probable outer membrane component of a multidrug efflux pump).

The SVM model identified some SNPs that are from known genes involved in DNA repair (mutL), antibiotic resistance (mexK, pirA, gyrA), host immune response evasion ( $w b p M)$, and host adaptation (yecS). Several mutations were found in a putative dipeptidase, PA2393, at positions 21 and 22 in the amino acid sequence. Several mutations identified by the SVM had low frequencies in the population; for example, V128A in pirA was only found in 29/947 CF isolates, and 9/631 non-CF. Some mutations found in hypothetical proteins include A137V in PA3923, V304A in probable major facilitator superfamily transporter PA2701, D172N in probable acyl-CoA dehydrogenase PA1631, A72R in PA3057, and S4F in probable ATP-dependent RNA helicase PA3466. These mutations were ranked 10-14 based on their absolute hyperplane coefficients. SVM was the only model to identify D87Y in gyrA, a mutation that has been found to increase drug resistance by altering the binding site of quinolones. The SVM ranked this mutation quite high $(18 / 394)$, which gives us more confidence in the SVM results.

To validate these findings, simulations can be run with created datasets to observe reproducibility, confirm accuracy of models and their gene/SNP predictions. Experimentally, gene knockouts for top hypothetical proteins could be performed in the lab, to test the prediction that these genes influence CF-pathoadaptive phenotypes. Where genes are missing in lab strains, gene knock-ins could be performed to identify protein function and changes to the phenotype. Predictive modeling tools could be used prior to experimental tests to predict protein function and provide guidance as to possible phenotypic changes. For SNPs, competition assays could be run in various environments (including a pseudo-CF lung environment) to measure changes in fitness and potentially indicate drivers behind these evolutionary changes. Additionally, the genomes of PA isolated during longitudinal studies following a single patient could be tested to see how these genes/SNPs change over the course of an infection. To experimentally compare results between studies, hypothetical proteins in the top 10-15 results of each method could be determined through experimental gene knockouts. The results from these experiments could indicate which algorithms had the fewest false positives, and therefore the best results.

In this study, I have discovered that RF and SVM models were able to identify variants in genes that have been previously described in the literature as contributing to PA pathoadaptation, as well as hypothetical proteins that we can research further. Proteins involved in metabolism, alginate 
pathways, antibiotic resistance and DNA repair were some of the highest ranked proteins in these methods. A number of the results from these methods overlapped with each other or PLINK. TreeWAS often recovered more hypothetical proteins than the other methods, in the most important genes. PLINK also identified a number of hypothetical proteins, but many of the top results were also found with RF and SVM models. PLINK likely had many false positive results, based on the QQ plots, identifying hundreds of genes and SNPs below the significance threshold.

In a broader sense, these results could be used in the future to aid in treatment of CF infections. For example, if some hypothetical proteins are characterized as contributing to antibiotic resistance, it could help inform physicians on which drug combination is best to combat the development of AMR. If there are proteins related to metabolism and biosynthesis pathways, there may be other treatment methods for the infections. Algorithms such as decision trees could provide diagnostic tools for PA infections, and help predict the spread of the bacterium in a population or within a patient. Machine learning, in general, could be used for more bacterial GWAS to find variants associated with a phenotype, even if the phenotype is rare. Some of these algorithms could also be used to see if there is a difference in genotype in differing severities of infection.

In conclusion, machine learning methods can provide insights into large genome datasets. Different models (RF, SVM or other algorithms) may be appropriate for various datasets, and multiple methods should be used to compare results and determine the most robust results. Several genes and mutations, both known and unknown, may contribute to CF lung adaption of Pseudomonas aeruginosa. These variants should be investigated further for their possible role in chronic PA infections. 


\section{REFERENCES}

[1] G. L. Winsor, E. J. Griffiths, R. Lo, B. K. Dhillon, J. A. Shay, and F. S. Brinkman, “Enhanced annotations and features for comparing thousands of Pseudomonas genomes in the Pseudomonas genome database," Nucleic Acids Research, vol. 44, no. D1, pp. 646-53, 2016. [Online]. Available: https://www.ncbi.nlm.nih.gov/pubmed/26578582

[2] J. L. Bobadilla, M. Macek Jr., J. P. Fine, and P. M. Farrell, "Cystic fibrosis: a worldwide analysis of CFTR mutations-correlation with incidence data and application to screening," Human Mutations, vol. 19, no. 6, pp. 575-606, 2002. [Online]. Available: https://www.ncbi.nlm.nih.gov/pubmed/12007216

[3] Cystic Fibrosis Canada, “The Canadian Cystic Fibrosis Registry 2017 Annual Data Report," Tech. Rep., 2017. [Online]. Available: https://www.cysticfibrosis.ca/uploads/ RegistryReport2017/2017RegistryAnnualDataReport.pdf

[4] J. C. Davies, E. W. F. W. Alton, and A. Bush, "Cystic fibrosis." BMJ (Clinical research ed.), vol. 335, no. 7632, pp. 1255-9, 2007. [Online]. Available: http: //www.ncbi.nlm.nih.gov/pubmed/18079549

[5] R. H. Keogh, S. R. Seaman, J. K. Barrett, D. Taylor-Robinson, and R. Szczesniak, "Dynamic prediction of survival in cystic fibrosis: A landmarking analysis using UK patient registry data." Epidemiology, vol. 30, no. 1, pp. 29-37, 2019. [Online]. Available: http://www.ncbi.nlm.nih.gov/pubmed/30234550

[6] A. Folkesson, L. Jelsbak, L. Yang, H. K. Johansen, O. Ciofu, N. Høiby, and S. Molin, "Adaptation of Pseudomonas aeruginosa to the cystic fibrosis airway: an evolutionary perspective," Nature Reviews Microbiology, vol. 10, no. 12, pp. 841-51, 2012. [Online]. Available: http://www.nature.com/articles/nrmicro2907

[7] A. M. Sousa and M. O. Pereira, "Pseudomonas aeruginosa diversification during infection development in cystic fibrosis lungs - A review." Pathogens, vol. 3, no. 3, pp. 680-703, 2014. [Online]. Available: http://www.ncbi.nlm.nih.gov/pubmed/25438018

[8] A. L. Cooney, P. B. McCray, and P. L. Sinn, "Cystic fibrosis gene therapy: Looking back, looking forward." Genes, vol. 9, no. 11, p. 538, 2018. [Online]. Available: http://www.ncbi.nlm.nih.gov/pubmed/30405068

[9] C. Edmondson and J. C. Davies, "Current and future treatment options for cystic fibrosis lung disease: latest evidence and clinical implications." Therapeutic Advances in Chronic Disease, vol. 7, no. 3, pp. 170-83, 2016. [Online]. Available: http://www.ncbi.nlm.nih.gov/pubmed/27347364

[10] J. B. Lyczak, C. L. Cannon, and G. B. Pier, "Lung infections associated with cystic fibrosis," Clinical Microbiology Reviews, vol. 15, no. 2, pp. 194-222, 2002. [Online]. Available: https://www.ncbi.nlm.nih.gov/pubmed/11932230

[11] A. Mena, E. E. Smith, J. L. Burns, D. P. Speert, S. M. Moskowitz, J. L. Perez, and A. Oliver, "Genetic adaptation of Pseudomonas aeruginosa to the airways of cystic fibrosis patients is catalyzed by hypermutation," Journal of Bacteriology, vol. 190, no. 24, pp. 7910-7, 2008. [Online]. Available: http://jb.asm.org/cgi/doi/10.1128/JB.01147-08

[12] P. B. Davis, “Cystic Fibrosis Since 1938," American Journal of Respiratory and Critical Care Medicine, vol. 173, no. 5, pp. 475-82, 2006. [Online]. Available: http://www.atsjournals.org/doi/abs/10.1164/rccm.200505-840OE 
[13] B. Coburn, P. W. Wang, J. Diaz Caballero, S. T. Clark, V. Brahma, S. Donaldson, Y. Zhang, A. Surendra, Y. Gong, D. Elizabeth Tullis, Y. C. W. Yau, V. J. Waters, D. M. Hwang, and D. S. Guttman, "Lung microbiota across age and disease stage in cystic fibrosis." Scientific Reports, vol. 5, p. 10241, 2015. [Online]. Available: http://www.ncbi.nlm.nih.gov/pubmed/25974282

[14] C. Harmer, K. Alnassafi, H. Hu, M. Elkins, P. Bye, B. Rose, S. Cordwell, J. A. Triccas, C. Harbour, and J. Manos, "Modulation of gene expression by Pseudomonas aeruginosa during chronic infection in the adult cystic fibrosis lung," Microbiology, vol. 159, pp. 2354-63, 2013. [Online]. Available: http://mic.microbiologyresearch.org/content/journal/micro/10. 1099/mic.0.066985-0

[15] M. Hogardt and J. Heesemann, "Microevolution of Pseudomonas aeruginosa to a chronic pathogen of the cystic fibrosis lung," Current Topics in Microbiology and Immunology, vol. 358, pp. 91-118, 2013. [Online]. Available: https://www.ncbi.nlm.nih.gov/pubmed/22311171

[16] J. Diaz Caballero, S. T. Clark, B. Coburn, Y. Zhang, P. W. Wang, S. L. Donaldson, D. E. Tullis, Y. C. W. Yau, V. J. Waters, D. M. Hwang, and D. S. Guttman, "Selective sweeps and parallel pathoadaptation drive Pseudomonas aeruginosa evolution in the cystic fibrosis lung." mBio, vol. 6, no. 5, 2015. [Online]. Available: http://www.ncbi.nlm.nih.gov/pubmed/26330513

[17] I. Bianconi, S. D'Arcangelo, A. Esposito, M. Benedet, E. Piffer, G. Dinnella, P. Gualdi, M. Schinella, E. Baldo, C. Donati, and O. Jousson, "Persistence and microevolution of Pseudomonas aeruginosa in the cystic fibrosis lung: A single-patient longitudinal genomic study." Frontiers in Microbiology, vol. 9, p. 3242, 2018. [Online]. Available: http://www.ncbi.nlm.nih.gov/pubmed/30692969

[18] S. E. Darch, A. McNally, F. Harrison, J. Corander, H. L. Barr, K. Paszkiewicz, S. Holden, A. Fogarty, S. A. Crusz, and S. P. Diggle, "Recombination is a key driver of genomic and phenotypic diversity in a Pseudomonas aeruginosa population during cystic fibrosis infection." Scientific Reports, vol. 5, p. 7649, 2015. [Online]. Available: http://www.ncbi.nlm.nih.gov/pubmed/25578031

[19] M. L. Workentine, C. D. Sibley, B. Glezerson, S. Purighalla, J. C. Norgaard-Gron, M. D. Parkins, H. R. Rabin, and M. G. Surette, "Phenotypic heterogeneity of Pseudomonas aeruginosa populations in a cystic fibrosis patient." PLoS One, vol. 8, no. 4, 2013. [Online]. Available: http://www.ncbi.nlm.nih.gov/pubmed/23573242

[20] J. Jeukens, B. Boyle, I. Kukavica-Ibrulj, M. M. Ouellet, S. D. Aaron, S. J. Charette, J. L. Fothergill, N. P. Tucker, C. Winstanley, and R. C. Levesque, "Comparative genomics of isolates of a Pseudomonas aeruginosa epidemic strain associated with chronic lung infections of cystic fibrosis patients." PLoS One, vol. 9, no. 2, 2014. [Online]. Available: http://www.ncbi.nlm.nih.gov/pubmed/24505294

[21] D. Williams, B. Evans, S. Haldenby, M. J. Walshaw, M. A. Brockhurst, C. Winstanley, and S. Paterson, "Divergent, coexisting Pseudomonas aeruginosa lineages in chronic cystic fibrosis lung infections." American Journal of Respiratory and Critical Care Medicine, vol. 191, no. 7, pp. 775-85, 2015. [Online]. Available: http://www.ncbi.nlm.nih.gov/pubmed/25590983

[22] E. E. Smith, D. G. Buckley, Z. Wu, C. Saenphimmachak, L. R. Hoffman, D. A. D'Argenio, S. I. Miller, B. W. Ramsey, D. P. Speert, S. M. Moskowitz, J. L. Burns, R. Kaul, and M. V. Olson, "Genetic adaptation by Pseudomonas aeruginosa to the airways of cystic fibrosis patients." Proceedings of the National Academy of Sciences of the United States of America, vol. 103, no. 22, pp. 8487-92, 2006. [Online]. Available: http://www.ncbi.nlm.nih.gov/pubmed/16687478 
[23] R. L. Marvig, L. M. Sommer, S. Molin, and H. K. Johansen, "Convergent evolution and adaptation of Pseudomonas aeruginosa within patients with cystic fibrosis," Nature Genetics, vol. 47, no. 1, pp. 57-64, 2015. [Online]. Available: http://www.nature.com/articles/ng.3148

[24] M. Hogardt, "Adaptation of Pseudomonas aeruginosa during persistence in the cystic fibrosis lung," International Journal of Medical Microbiology, vol. 300, no. 8, pp. 557-62, 2010. [Online]. Available: https://www.sciencedirect.com/science/article/pii/S1438422110000883? via\%3Dihub

[25] R. A. Proctor, C. von Eiff, B. C. Kahl, K. Becker, P. McNamara, M. Herrmann, and G. Peters, "Small colony variants: a pathogenic form of bacteria that facilitates persistent and recurrent infections," Nature Reviews Microbiology, vol. 4, no. 4, pp. 295-305, 2006. [Online]. Available: http://www.ncbi.nlm.nih.gov/pubmed/16541137

[26] T.-F. Mah, B. Pitts, B. Pellock, G. C. Walker, P. S. Stewart, and G. A. O’Toole, "A genetic basis for Pseudomonas aeruginosa biofilm antibiotic resistance," Nature, vol. 426, no. 6964, pp. 306-10, 2003. [Online]. Available: http://www.ncbi.nlm.nih.gov/pubmed/14628055

[27] L. Zhang, M. Fritsch, L. Hammond, R. Landreville, C. Slatculescu, A. Colavita, and T.-F. Mah, "Identification of genes involved in Pseudomonas aeruginosa biofilmspecific resistance to antibiotics." PLoS One, vol. 8, no. 4, 2013. [Online]. Available: http://www.ncbi.nlm.nih.gov/pubmed/23637868

[28] P. K. Taylor, L. Zhang, and T.-F. Mah, "Loss of the two-component system TctD-TctE in Pseudomonas aeruginosa affects biofilm formation and aminoglycoside susceptibility in response to citric acid." mSphere, vol. 4, no. 2, 2019. [Online]. Available: http://www.ncbi.nlm.nih.gov/pubmed/30842268

[29] J. M. Munita and C. A. Arias, "Mechanisms of antibiotic resistance." Microbiology Spectrum, vol. 4, no. 2, 2016. [Online]. Available: http://www.ncbi.nlm.nih.gov/pubmed/27227291

[30] K. Poonsuk, C. Tribuddharat, and R. Chuanchuen, "Simultaneous overexpression of multidrug efflux pumps in Pseudomonas aeruginosa non-cystic fibrosis clinical isolates," Canadian Journal of Microbiology, vol. 60, no. 7, pp. 437-43, 2014. [Online]. Available: http://www.nrcresearchpress.com/doi/pdf/10.1139/cjm-2014-0239

[31] R. Nouri, M. Ahangarzadeh Rezaee, A. Hasani, M. Aghazadeh, and M. Asgharzadeh, "The role of gyrA and parC mutations in fluoroquinolones-resistant Pseudomonas aeruginosa isolates from Iran." Brazilian journal of microbiology : [publication of the Brazilian Society for Microbiology], vol. 47, no. 4, pp. 925-30, 2016. [Online]. Available: http://www.ncbi.nlm.nih.gov/pubmed/27522930

[32] S. Jalal, O. Ciofu, N. Hoiby, N. Gotoh, and B. Wretlind, "Molecular mechanisms of fluoroquinolone resistance in Pseudomonas aeruginosa isolates from cystic fibrosis patients." Antimicrobial Agents and Chemotherapy, vol. 44, no. 3, pp. 710-2, 2000. [Online]. Available: http://www.ncbi.nlm.nih.gov/pubmed/10681343

[33] J. C. S. Chung, J. Becq, L. Fraser, O. Schulz-Trieglaff, N. J. Bond, J. Foweraker, K. D. Bruce, G. P. Smith, and M. Welch, "Genomic variation among contemporary Pseudomonas aeruginosa isolates from chronically infected cystic fibrosis patients." Journal of Bacteriology, vol. 194, no. 18, pp. 4857-66, 9 2012. [Online]. Available: http://www.ncbi.nlm.nih.gov/pubmed/22753054

[34] M. Knopp and D. I. Andersson, "Predictable phenotypes of antibiotic resistance mutations." mBio, vol. 9, no. 3, 2018. [Online]. Available: http://www.ncbi.nlm.nih.gov/pubmed/29764951 
[35] J. Jeukens, I. Kukavica-Ibrulj, J. G. Emond-Rheault, L. Freschi, and R. C. Levesque, "Comparative genomics of a drug-resistant Pseudomonas aeruginosa panel and the challenges of antimicrobial resistance prediction from genomes," FEMS Microbiology Letters, vol. 364, no. 18, 2017. [Online]. Available: https://academic.oup.com/femsle/article/doi/10.1093/ femsle/fnx161/4056142

[36] G. Cabot, A. A. Ocampo-Sosa, F. Tubau, M. D. Macia, C. Rodríguez, B. Moya, L. Zamorano, C. Suárez, C. Peña, L. Martínez-Martínez, A. Oliver, and Spanish Network for Research in Infectious Diseases (REIPI), "Overexpression of AmpC and efflux pumps in Pseudomonas aeruginosa isolates from bloodstream infections: prevalence and impact on resistance in a Spanish multicenter study." Antimicrobial Agents and Chemotherapy, vol. 55, no. 5, pp. 1906-11, 2011. [Online]. Available: http://www.ncbi.nlm.nih.gov/pubmed/21357294

[37] B. Valot, C. Guyeux, J. Y. Rolland, K. Mazouzi, X. Bertrand, and D. Hocquet, "What it takes to be a Pseudomonas aeruginosa? The core genome of the opportunistic pathogen updated," PLoS One, vol. 10, no. 5, 2015. [Online]. Available: https://www.ncbi.nlm.nih.gov/pubmed/25961859

[38] P. H. Roy, S. G. Tetu, A. Larouche, L. Elbourne, S. Tremblay, Q. Ren, R. Dodson, D. Harkins, R. Shay, K. Watkins, Y. Mahamoud, and I. T. Paulsen, "Complete genome sequence of the multiresistant taxonomic outlier Pseudomonas aeruginosa PA7," PLoS One, vol. 5, no. 1, 2010. [Online]. Available: http://www.ncbi.nlm.nih.gov/pubmed/20107499

[39] E. A. Ozer, J. P. Allen, and A. R. Hauser, "Characterization of the core and accessory genomes of Pseudomonas aeruginosa using bioinformatic tools Spine and AGEnt," BMC Genomics, vol. 15, p. 737, 2014. [Online]. Available: https://www.ncbi.nlm.nih.gov/pubmed/25168460

[40] J. Klockgether, N. Cramer, L. Wiehlmann, C. F. Davenport, and B. Tümmler, "Pseudomonas aeruginosa genomic structure and diversity." Frontiers in Microbiology, vol. 2, p. 150, 2011. [Online]. Available: http://www.ncbi.nlm.nih.gov/pubmed/21808635

[41] K. Mathee, G. Narasimhan, C. Valdes, X. Qiu, J. M. Matewish, M. Koehrsen, A. Rokas, C. N. Yandava, R. Engels, E. Zeng, R. Olavarietta, M. Doud, R. S. Smith, P. Montgomery, J. R. White, P. A. Godfrey, C. Kodira, B. Birren, J. E. Galagan, and S. Lory, “Dynamics of Pseudomonas aeruginosa genome evolution." Proceedings of the National Academy of Sciences of the United States of America, vol. 105, no. 8, pp. 3100-5, 2008. [Online]. Available: http://www.ncbi.nlm.nih.gov/pubmed/18287045

[42] L. Freschi, A. T. Vincent, J. Jeukens, J.-G. Emond-Rheault, I. Kukavica-Ibrulj, M.-J. Dupont, S. J. Charette, B. Boyle, and R. C. Levesque, "The Pseudomonas aeruginosa pan-genome provides new insights on its population structure, horizontal gene transfer, and pathogenicity," Genome Biology and Evolution, vol. 11, no. 1, pp. 109-20, 2019. [Online]. Available: https://academic.oup.com/gbe/article/11/1/109/5215156

[43] J. Mosquera-Rendón, A. M. Rada-Bravo, S. Cárdenas-Brito, M. Corredor, E. Restrepo-Pineda, and A. Benítez-Páez, "Pangenome-wide and molecular evolution analyses of the Pseudomonas aeruginosa species," BMC Genomics, vol. 17, no. 1, p. 45, 2016. [Online]. Available: http://www.ncbi.nlm.nih.gov/pubmed/26754847

[44] H. Tettelin, V. Masignani, M. J. Cieslewicz, C. Donati, D. Medini, N. L. Ward, S. V. Angiuoli, J. Crabtree, A. L. Jones, A. S. Durkin, R. T. Deboy, T. M. Davidsen, M. Mora, M. Scarselli, I. Margarit y Ros, J. D. Peterson, C. R. Hauser, J. P. Sundaram, W. C. Nelson, R. Madupu, L. M. Brinkac, R. J. Dodson, M. J. Rosovitz, S. A. Sullivan, S. C. Daugherty, D. H. Haft, J. Selengut, M. L. Gwinn, L. Zhou, N. Zafar, H. Khouri, D. Radune, 
G. Dimitrov, K. Watkins, K. J. B. O'Connor, S. Smith, T. R. Utterback, O. White, C. E. Rubens, G. Grandi, L. C. Madoff, D. L. Kasper, J. L. Telford, M. R. Wessels, R. Rappuoli, and C. M. Fraser, "Genome analysis of multiple pathogenic isolates of Streptococcus agalactiae: implications for the microbial "pan-genome"," Proceedings of the National Academy of Sciences of the United States of America, vol. 102, no. 39, pp. 13950-5, 2005. [Online]. Available: http://www.ncbi.nlm.nih.gov/pubmed/16172379

[45] A. J. van Tonder, S. Mistry, J. E. Bray, D. M. C. Hill, A. J. Cody, C. L. Farmer, K. P. Klugman, A. von Gottberg, S. D. Bentley, J. Parkhill, K. A. Jolley, M. C. J. Maiden, and A. B. Brueggemann, "Defining the estimated core genome of bacterial populations using a Bayesian decision model." PLoS Computational Biology, vol. 10, no. 8, 2014. [Online]. Available: http://www.ncbi.nlm.nih.gov/pubmed/25144616

[46] A. Buniello, J. MacArthur, M. Cerezo, L. Harris, J. Hayhurst, C. Malangone, A. McMahon, J. Morales, E. Mountjoy, E. Sollis, D. Suveges, O. Vrousgou, P. Whetzel, R. Amode, J. Guillen, H. Riat, S. Trevanion, P. Hall, H. Junkins, P. Flicek, T. Burdett, L. Hindorff, F. Cunningham, and H. Parkinson, "The NHGRI-EBI Catalog of published genome-wide association studies," Nucleic Acids Research, vol. 47, 2019. [Online]. Available: www.ebi.ac.uk/gwas

[47] M. C. Mills and C. Rahal, "A scientometric review of genome-wide association studies," Communications Biology, vol. 2, no. 1, 2019. [Online]. Available: http: //www.nature.com/articles/s42003-018-0261-x

[48] S. Purcell, B. Neale, K. Todd-Brown, L. Thomas, M. A. R. Ferreira, D. Bender, J. Maller, P. Sklar, P. I. W. de Bakker, M. J. Daly, and P. C. Sham, "PLINK: a tool set for whole-genome association and population-based linkage analyses." American Journal of Human Genetics, vol. 81, no. 3, pp. 559-75, 2007. [Online]. Available: http://www.ncbi.nlm.nih.gov/pubmed/17701901

[49] C. C. Chang, C. C. Chow, L. C. Tellier, S. Vattikuti, S. M. Purcell, and J. J. Lee, "Second-generation PLINK: rising to the challenge of larger and richer datasets," Gigascience, vol. 4, no. 7, 2015. [Online]. Available: https://www.ncbi.nlm.nih.gov/pubmed/25722852

[50] C. Wu, A. DeWan, J. Hoh, and Z. Wang, "A comparison of association methods correcting for population stratification in case-control studies." Annals of Human Genetics, vol. 75, no. 3, pp. 418-27, 2011. [Online]. Available: http://www.ncbi.nlm.nih.gov/pubmed/21281271

[51] N. A. Rosenberg, L. Huang, E. M. Jewett, Z. A. Szpiech, I. Jankovic, and M. Boehnke, “Genomewide association studies in diverse populations," Nature Reviews Genetics, vol. 11, no. 5, pp. 356-66, 2010. [Online]. Available: https://www.ncbi.nlm.nih.gov/pubmed/20395969

[52] N. Patterson, A. L. Price, and D. Reich, "Population structure and eigenanalysis." PLoS Genetics, vol. 2, no. 12, 2006. [Online]. Available: http://www.ncbi.nlm.nih.gov/pubmed/17194218

[53] A. L. Price, N. J. Patterson, R. M. Plenge, M. E. Weinblatt, N. A. Shadick, and D. Reich, "Principal components analysis corrects for stratification in genome-wide association studies," Nature Genetics, vol. 38, no. 8, pp. 904-9, 2006. [Online]. Available: https://www.ncbi.nlm.nih.gov/pubmed/16862161

[54] A. Raj, M. Stephens, and J. K. Pritchard, "fastSTRUCTURE: variational inference of population structure in large SNP data sets." Genetics, vol. 197, no. 2, pp. 573-89, 2014. [Online]. Available: http://www.ncbi.nlm.nih.gov/pubmed/24700103

[55] D. H. Alexander and K. Lange, "Enhancements to the ADMIXTURE algorithm for individual ancestry estimation," BMC Bioinformatics, vol. 12, no. 1, p. 246, 2011. [Online]. Available: https://bmcbioinformatics.biomedcentral.com/articles/10.1186/1471-2105-12-246 
[56] H. J. Cordell, "Detecting gene-gene interactions that underlie human diseases," Nature Reviews Genetics, vol. 10, no. 6, pp. 392-404, 2009. [Online]. Available: https://www.ncbi.nlm.nih.gov/pubmed/19434077

[57] C. A. Anderson, F. H. Pettersson, G. M. Clarke, L. R. Cardon, A. P. Morris, and K. T. Zondervan, "Data quality control in genetic case-control association studies." Nature Protocols, vol. 5, no. 9, pp. 1564-73, 2010. [Online]. Available: http://www.ncbi.nlm.nih.gov/pubmed/21085122

[58] M. I. McCarthy, G. R. Abecasis, L. R. Cardon, D. B. Goldstein, J. Little, J. P. Ioannidis, and J. N. Hirschhorn, "Genome-wide association studies for complex traits: consensus, uncertainty and challenges," Nature Reviews Genetics, vol. 9, no. 5, pp. 356-69, 2008. [Online]. Available: https://www.ncbi.nlm.nih.gov/pubmed/18398418

[59] C. Fang and J. Luo, "Metabolic GWAS-based dissection of genetic bases underlying the diversity of plant metabolism," The Plant Journal, vol. 97, no. 1, pp. 91-100, 2019. [Online]. Available: http://www.ncbi.nlm.nih.gov/pubmed/30231195

[60] A. C. Gerstein, K. M. Jackson, T. R. McDonald, Y. Wang, B. D. Lueck, S. Bohjanen, K. D. Smith, A. Akampurira, D. B. Meya, C. Xue, D. R. Boulware, and K. Nielsen, "Identification of pathogen genomic differences that impact human immune response and disease during Cryptococcus neoformans infection," bioRxiv, 3 2019. [Online]. Available: https://www.biorxiv.org/content/10.1101/592212v1.full

[61] P. E. Chen and B. J. Shapiro, "The advent of genome-wide association studies for bacteria," Current Opinion in Microbiology, vol. 25, pp. 17-24, 2015. [Online]. Available: https://www.ncbi.nlm.nih.gov/pubmed/25835153

[62] R. A. Power, J. Parkhill, and T. de Oliveira, "Microbial genome-wide association studies: lessons from human GWAS," Nature Reviews Genetics, vol. 18, no. 1, pp. 41-50, 2017. [Online]. Available: https://www.ncbi.nlm.nih.gov/pubmed/27840430

[63] S. G. Earle, C. H. Wu, J. Charlesworth, N. Stoesser, N. C. Gordon, T. M. Walker, C. C. A. Spencer, Z. Iqbal, D. A. Clifton, K. L. Hopkins, N. Woodford, E. G. Smith, N. Ismail, M. J. Llewelyn, T. E. Peto, D. W. Crook, G. McVean, A. S. Walker, and D. J. Wilson, "Identifying lineage effects when controlling for population structure improves power in bacterial association studies," Nature Microbiology, vol. 1, p. 16041, 2016. [Online]. Available: https://www.ncbi.nlm.nih.gov/pubmed/27572646

[64] O. Miotto, R. Amato, E. A. Ashley, B. MacInnis, J. Almagro-Garcia, C. Amaratunga, P. Lim, D. Mead, S. O. Oyola, M. Dhorda, M. Imwong, C. Woodrow, M. Manske, J. Stalker, E. Drury, S. Campino, L. Amenga-Etego, T.-N. N. Thanh, H. T. Tran, P. Ringwald, D. Bethell, F. Nosten, A. P. Phyo, S. Pukrittayakamee, K. Chotivanich, C. M. Chuor, C. Nguon, S. Suon, S. Sreng, P. N. Newton, M. Mayxay, M. Khanthavong, B. Hongvanthong, Y. Htut, K. T. Han, M. P. Kyaw, M. A. Faiz, C. I. Fanello, M. Onyamboko, O. A. Mokuolu, C. G. Jacob, S. Takala-Harrison, C. V. Plowe, N. P. Day, A. M. Dondorp, C. C. A. Spencer, G. McVean, R. M. Fairhurst, N. J. White, and D. P. Kwiatkowski, "Genetic architecture of artemisinin-resistant Plasmodium falciparum." Nature genetics, vol. 47, no. 3, pp. 226-34, 2015. [Online]. Available: http://www.ncbi.nlm.nih.gov/pubmed/25599401

[65] M. J. Skwark, N. J. Croucher, S. Puranen, C. Chewapreecha, M. Pesonen, Y. Y. Xu, P. Turner, S. R. Harris, S. B. Beres, J. M. Musser, J. Parkhill, S. D. Bentley, E. Aurell, and J. Corander, "Interacting networks of resistance, virulence and core machinery genes identified by genome-wide epistasis analysis." PLoS Genetics, vol. 13, no. 2, 2017. [Online]. Available: http://www.ncbi.nlm.nih.gov/pubmed/28207813 
[66] S. Puranen, M. Pesonen, J. Pensar, Y. Y. Xu, J. A. Lees, S. D. Bentley, N. J. Croucher, and J. Corander, "SuperDCA for genome-wide epistasis analysis." Microbial Genomics, vol. 4, no. 6, 2018. [Online]. Available: http://www.ncbi.nlm.nih.gov/pubmed/29813016

[67] C. Collins and X. Didelot, "A phylogenetic method to perform genome-wide association studies in microbes that accounts for population structure and recombination," PLoS Computational Biology, vol. 14, no. 2, 2018. [Online]. Available: https: //dx.plos.org/10.1371/journal.pcbi.1005958

[68] M. R. Farhat, B. J. Shapiro, S. K. Sheppard, C. Colijn, and M. Murray, “A phylogeny-based sampling strategy and power calculator informs genome-wide associations study design for microbial pathogens," Genome Medicine, vol. 6, no. 11, pp. 1-14, 2014. [Online]. Available: https://www.ncbi.nlm.nih.gov/pmc/articles/PMC4256898/

[69] L. Proctor, "Priorities for the next 10 years of human microbiome research," Nature, vol. 569, pp. 623-5, 2019. [Online]. Available: http://www.nature.com/articles/d41586-019-01654-0

[70] A. E. Darling, B. Mau, and N. T. Perna, "progressiveMauve: Multiple genome alignment with gene gain, loss and rearrangement," PLoS One, vol. 5, no. 6, 2010. [Online]. Available: https://dx.plos.org/10.1371/journal.pone.0011147

[71] S. J. Salipante, D. J. Roach, J. O. Kitzman, M. W. Snyder, B. Stackhouse, S. M. Butler-Wu, C. Lee, B. T. Cookson, and J. Shendure, "Large-scale genomic sequencing of extraintestinal pathogenic Escherichia coli strains." Genome Research, vol. 25, no. 1, pp. 119-28, 2015. [Online]. Available: http://www.ncbi.nlm.nih.gov/pubmed/25373147

[72] M. R. Farhat, B. J. Shapiro, K. J. Kieser, R. Sultana, K. R. Jacobson, T. C. Victor, R. M. Warren, E. M. Streicher, A. Calver, A. Sloutsky, D. Kaur, J. E. Posey, B. Plikaytis, M. R. Oggioni, J. L. Gardy, J. C. Johnston, M. Rodrigues, P. K. Tang, M. Kato-Maeda, M. L. Borowsky, B. Muddukrishna, B. N. Kreiswirth, N. Kurepina, J. Galagan, S. Gagneux, B. Birren, E. J. Rubin, E. S. Lander, P. C. Sabeti, and M. Murray, "Genomic analysis identifies targets of convergent positive selection in drug-resistant Mycobacterium tuberculosis," Nature Genetics, vol. 45, no. 10, pp. 1183-9, 2013. [Online]. Available: http://dx.doi.org/10.1038/ng.2747

[73] S. K. Sheppard, X. Didelot, G. Meric, A. Torralbo, K. A. Jolley, D. J. Kelly, S. D. Bentley, M. C. Maiden, J. Parkhill, and D. Falush, "Genome-wide association study identifies vitamin B5 biosynthesis as a host specificity factor in Campylobacter," Proceedings of the National Academy of Sciences of the United States of America, vol. 110, no. 29, pp. 11923-7, 2013. [Online]. Available: https://www.ncbi.nlm.nih.gov/pubmed/23818615

[74] S. van Hemert, M. Meijerink, D. Molenaar, P. A. Bron, P. de Vos, M. Kleerebezem, J. M. Wells, and M. L. Marco, "Identification of Lactobacillus plantarum genes modulating the cytokine response of human peripheral blood mononuclear cells." BMC Microbiology, vol. 10, p. 293, 2010. [Online]. Available: http://www.ncbi.nlm.nih.gov/pubmed/21080958

[75] N. Stoesser, E. M. Batty, D. W. Eyre, M. Morgan, D. H. Wyllie, C. Del Ojo Elias, J. R. Johnson, A. S. Walker, T. E. A. Peto, and D. W. Crook, "Predicting antimicrobial susceptibilities for Escherichia coli and Klebsiella pneumoniae isolates using whole genomic sequence data." Journal of Antimicrobial Chemotherapy, vol. 68, no. 10, pp. 2234-44, 2013. [Online]. Available: http://www.ncbi.nlm.nih.gov/pubmed/23722448

[76] M. T. Alam, R. A. Petit, E. K. Crispell, T. A. Thornton, K. N. Conneely, Y. Jiang, S. W. Satola, T. D. Read, R. A. Petit 3rd, E. K. Crispell, T. A. Thornton, K. N. Conneely, Y. Jiang, S. W. Satola, and T. D. Read, "Dissecting vancomycin-intermediate resistance in staphylococcus 
aureus using genome-wide association," Genome Biology and Evolution, vol. 6, no. 5, pp. 1174-85, 2014. [Online]. Available: https://www.ncbi.nlm.nih.gov/pubmed/24787619

[77] N. C. Gordon, J. R. Price, K. Cole, R. Everitt, M. Morgan, J. Finney, A. M. Kearns, B. Pichon, B. Young, D. J. Wilson, M. J. Llewelyn, J. Paul, T. E. A. Peto, D. W. Crook, A. S. Walker, and T. Golubchik, "Prediction of Staphylococcus aureus antimicrobial resistance by whole-genome sequencing." Journal of Clinical Microbiology, vol. 52, no. 4, pp. 1182-91, 2014. [Online]. Available: http://www.ncbi.nlm.nih.gov/pubmed/24501024

[78] M. Laabei, M. Recker, J. K. Rudkin, M. Aldeljawi, Z. Gulay, T. J. Sloan, P. Williams, J. L. Endres, K. W. Bayles, P. D. Fey, V. K. Yajjala, T. Widhelm, E. Hawkins, K. Lewis, S. Parfett, L. Scowen, S. J. Peacock, M. Holden, D. Wilson, T. D. Read, J. van den Elsen, N. K. Priest, E. J. Feil, L. D. Hurst, E. Josefsson, and R. C. Massey, "Predicting the virulence of MRSA from its genome sequence," Genome Research, vol. 24, no. 5, pp. 839-49, 2014. [Online]. Available: https://www.ncbi.nlm.nih.gov/pubmed/24717264

[79] C. Chewapreecha, P. Marttinen, N. J. Croucher, S. J. Salter, S. R. Harris, A. E. Mather, W. P. Hanage, D. Goldblatt, F. H. Nosten, C. Turner, P. Turner, S. D. Bentley, and J. Parkhill, "Comprehensive identification of single nucleotide polymorphisms associated with beta-lactam resistance within pneumococcal mosaic genes," PLoS Genetics, vol. 10, no. 8, 2014. [Online]. Available: https://www.ncbi.nlm.nih.gov/pubmed/25101644

[80] J. M. Chaston, P. D. Newell, and A. E. Douglas, "Metagenome-wide association of microbial determinants of host phenotype in Drosophila melanogaster." mBio, vol. 5, no. 5, 2014. [Online]. Available: http://www.ncbi.nlm.nih.gov/pubmed/25271286

[81] T. M. Walker, T. A. Kohl, S. V. Omar, J. Hedge, C. Del Ojo Elias, P. Bradley, Z. Iqbal, S. Feuerriegel, K. E. Niehaus, D. J. Wilson, D. A. Clifton, G. Kapatai, C. L. C. Ip, R. Bowden, F. A. Drobniewski, C. Allix-Béguec, C. Gaudin, J. Parkhill, R. Diel, P. Supply, D. W. Crook, E. G. Smith, A. S. Walker, N. Ismail, S. Niemann, T. E. A. Peto, and Modernizing Medical Microbiology (MMM) Informatics Group, "Whole-genome sequencing for prediction of Mycobacterium tuberculosis drug susceptibility and resistance: a retrospective cohort study." The Lancet. Infectious diseases, vol. 15, no. 10, pp. 1193-202, 2015. [Online]. Available: http://www.ncbi.nlm.nih.gov/pubmed/26116186

[82] C. A. Desjardins, K. A. Cohen, V. Munsamy, T. Abeel, K. Maharaj, B. J. Walker, T. P. Shea, D. V. Almeida, A. L. Manson, A. Salazar, N. Padayatchi, M. R. O'Donnell, K. P. Mlisana, J. Wortman, B. W. Birren, J. Grosset, A. M. Earl, and A. S. Pym, "Genomic and functional analyses of Mycobacterium tuberculosis strains implicate ald in D-cycloserine resistance," Nature Genetics, vol. 48, no. 5, pp. 544-51, 2016. [Online]. Available: https://www.ncbi.nlm.nih.gov/pubmed/27064254

[83] K. A. Cohen, T. El-Hay, K. L. Wyres, O. Weissbrod, V. Munsamy, C. Yanover, R. Aharonov, O. Shaham, T. C. Conway, Y. Goldschmidt, W. R. Bishai, and A. S. Pym, "Paradoxical hypersusceptibility of drug-resistant Mycobacterium tuberculosis to $\beta$-lactam antibiotics." EBioMedicine, vol. 9, pp. 170-9, 2016. [Online]. Available: http://www.ncbi.nlm.nih.gov/pubmed/27333036

[84] J. A. Lees, M. Vehkala, N. Välimäki, S. R. Harris, C. Chewapreecha, N. J. Croucher, P. Marttinen, M. R. Davies, A. C. Steer, S. Y. C. Tong, A. Honkela, J. Parkhill, S. D. Bentley, and J. Corander, "Sequence element enrichment analysis to determine the genetic basis of bacterial phenotypes." Nature Communications, vol. 7, p. 12797, 2016. [Online]. Available: http://www.ncbi.nlm.nih.gov/pubmed/27633831 
[85] M. Suzuki, K. Shibayama, and K. Yahara, "A genome-wide association study identifies a horizontally transferred bacterial surface adhesin gene associated with antimicrobial resistant strains," Scientific Reports, vol. 6, pp. 1-10, 2016. [Online]. Available: http://dx.doi.org/10.1038/srep37811

[86] Y.-J. Bao, B. J. Shapiro, S. W. Lee, V. A. Ploplis, and F. J. Castellino, "Phenotypic differentiation of Streptococcus pyogenes populations is induced by recombination-driven gene-specific sweeps," Scientific Reports, vol. 6, p. 36644, 2016. [Online]. Available: http://www.ncbi.nlm.nih.gov/pubmed/27821851

[87] S. S. Porter, P. L. Chang, C. A. Conow, J. P. Dunham, and M. L. Friesen, “Association mapping reveals novel serpentine adaptation gene clusters in a population of symbiotic Mesorhizobium." The ISME journal, vol. 11, no. 1, pp. 248-62, 2017. [Online]. Available: http://www.ncbi.nlm.nih.gov/pubmed/27420027

[88] P. Kremer, J. Lees, M. Koopmans, B. Ferwerda, A. Arends, M. Feller, K. Schipper, M. V. Seron, A. v. d. Ende, M. Brouwer, D. v. d. Beek, and S. Bentley, "Benzalkonium tolerance genes and outcome in Listeria monocytogenes meningitis," Clinical Microbiology and Infection, vol. 23, no. 4, 2017. [Online]. Available: http://www.ncbi.nlm.nih.gov/pubmed/27998823

[89] F. M. Mobegi, A. J. H. Cremers, M. I. de Jonge, S. D. Bentley, S. A. F. T. van Hijum, and A. Zomer, "Deciphering the distance to antibiotic resistance for the pneumococcus using genome sequencing data." Scientific Reports, vol. 7, p. 42808, 2017. [Online]. Available: http://www.ncbi.nlm.nih.gov/pubmed/28205635

[90] J. A. Lees, N. J. Croucher, D. Goldblatt, F. Nosten, J. Parkhill, C. Turner, P. Turner, and S. D. Bentley, "Genome-wide identification of lineage and locus specific variation associated with pneumococcal carriage duration." eLife, vol. 6, 2017. [Online]. Available: http://www.ncbi.nlm.nih.gov/pubmed/28742023

[91] T. Swings, B. Weytjens, T. Schalck, C. Bonte, N. Verstraeten, J. Michiels, and K. Marchal, "Network-based identification of adaptive pathways in evolved ethanol-tolerant bacterial populations." Molecular Biology and Evolution, vol. 34, no. 11, pp. 2927-43, 2017. [Online]. Available: http://www.ncbi.nlm.nih.gov/pubmed/28961727

[92] X. He, Y. Jin, M. Ye, N. Chen, J. Zhu, J. Wang, L. Jiang, and R. Wu, "Bacterial genetic architecture of ecological interactions in co-culture by GWAS-taking Escherichia coli and Staphylococcus aureus as an example," Frontiers in Microbiology, vol. 8, p. 2332, 2017. [Online]. Available: https://www.ncbi.nlm.nih.gov/pmc/articles/PMC5712204/

[93] E. Berthenet, K. Yahara, K. Thorell, B. Pascoe, G. Meric, J. M. Mikhail, L. Engstrand, H. Enroth, A. Burette, F. Megraud, C. Varon, J. C. Atherton, S. Smith, T. S. Wilkinson, M. D. Hitchings, D. Falush, and S. K. Sheppard, "A GWAS on Helicobacter pylori strains points to genetic variants associated with gastric cancer risk," BMC Biology, vol. 16, no. 84, 2018. [Online]. Available: https://bmcbiol.biomedcentral.com/articles/10.1186/s12915-018-0550-3

[94] F. Coll, J. Phelan, G. A. Hill-Cawthorne, M. B. Nair, K. Mallard, S. Ali, A. M. Abdallah, S. Alghamdi, M. Alsomali, A. O. Ahmed, S. Portelli, Y. Oppong, A. Alves, T. B. Bessa, S. Campino, M. Caws, A. Chatterjee, A. C. Crampin, K. Dheda, N. Furnham, J. R. Glynn, L. Grandjean, D. Minh Ha, R. Hasan, Z. Hasan, M. L. Hibberd, M. Joloba, E. C. Jones-López, T. Matsumoto, A. Miranda, D. J. Moore, N. Mocillo, S. Panaiotov, J. Parkhill, C. Penha, J. Perdigão, I. Portugal, Z. Rchiad, J. Robledo, P. Sheen, N. T. Shesha, F. A. Sirgel, C. Sola, E. Oliveira Sousa, E. M. Streicher, P. V. Helden, M. Viveiros, R. M. Warren, R. McNerney, A. Pain, and T. G. Clark, "Genome-wide analysis of multi- and extensively drug-resistant 
Mycobacterium tuberculosis," Nature Genetics, vol. 50, no. 2, pp. 307-16, 2018. [Online]. Available: http://www.nature.com/articles/s41588-017-0029-0

[95] N. E. Wheeler, P. P. Gardner, and L. Barquist, "Machine learning identifies signatures of host adaptation in the bacterial pathogen Salmonella enterica," PLoS Genetics, vol. 14, no. 5, 2018. [Online]. Available: http://www.ncbi.nlm.nih.gov/pubmed/29738521

[96] E. S. Kavvas, E. Catoiu, N. Mih, J. T. Yurkovich, Y. Seif, N. Dillon, D. Heckmann, A. Anand, L. Yang, V. Nizet, J. M. Monk, and B. O. Palsson, "Machine learning and structural analysis of Mycobacterium tuberculosis pan-genome identifies genetic signatures of antibiotic resistance." Nature Communications, vol. 9, no. 1, p. 4306, 2018. [Online]. Available: http://www.ncbi.nlm.nih.gov/pubmed/30333483

[97] J. Diaz Caballero, S. T. Clark, P. W. Wang, S. L. Donaldson, B. Coburn, D. E. Tullis, Y. C. W. Yau, V. J. Waters, D. M. Hwang, and D. S. Guttman, "A genome-wide association analysis reveals a potential role for recombination in the evolution of antimicrobial resistance in Burkholderia multivorans." PLoS Pathogens, vol. 14, no. 12, 2018. [Online]. Available: http://www.ncbi.nlm.nih.gov/pubmed/30532201

[98] B. Epstein, R. A. I. Abou-Shanab, A. Shamseldin, M. R. Taylor, J. Guhlin, L. T. Burghardt, M. Nelson, M. J. Sadowsky, and P. Tiffin, "Genome-wide association analyses in the model rhizobium Ensifer meliloti." mSphere, vol. 3, no. 5, 2018. [Online]. Available: http://www.ncbi.nlm.nih.gov/pubmed/30355664

[99] C. Ruesen, L. Chaidir, A. van Laarhoven, S. Dian, A. R. Ganiem, H. Nebenzahl-Guimaraes, M. A. Huynen, B. Alisjahbana, B. E. Dutilh, and R. van Crevel, "Large-scale genomic analysis shows association between homoplastic genetic variation in Mycobacterium tuberculosis genes and meningeal or pulmonary tuberculosis," BMC Genomics, vol. 19, no. 1, p. 122, 2018. [Online]. Available: http://www.ncbi.nlm.nih.gov/pubmed/29402222

[100] A. Gori, O. Harrison, E. Mlia, Y. Nishihara, J. Chinkwita-Phiri, M. Mallewa, Q. Dube, T. D. Swarthout, A. H. Nobbs, M. Maiden, N. French, and R. S. Heyderman, "Pan-GWAS of Streptococcus agalactiae highlights lineage-specific genes associated with virulence and niche adaptation," bioRxiv, p. 574152, 2019. [Online]. Available: https://www.biorxiv.org/content/10.1101/574152v1

[101] G. Buron-Moles, A. Chailyan, I. Dolejs, J. Forster, and M. H. Mikš, “Uncovering carbohydrate metabolism through a genotype-phenotype association study of 56 lactic acid bacteria genomes," Applied Microbiology and Biotechnology, vol. 103, no. 7, pp. 3135-52, 2019. [Online]. Available: http://www.ncbi.nlm.nih.gov/pubmed/30830251

[102] M. Zhang, Q. Su, Y. Lu, M. Zhao, and B. Niu, "Application of machine learning approaches for protein-protein interactions prediction," Medicinal Chemistry, vol. 13, no. 6, pp. 506-14, 2017. [Online]. Available: http://www.ncbi.nlm.nih.gov/pubmed/28530547

[103] L. Breiman, "Random forests," Machine Learning, vol. 45, no. 1, pp. 5-32, 2001. [Online]. Available: https://link.springer.com/article/10.1023/A:1010933404324

[104] C. J. Burges, "A tutorial on support vector machines for pattern recognition," Data Mining and Knowledge Discovery, vol. 2, no. 2, pp. 121-67, 1998. [Online]. Available: http://www.springerlink.com/index/Q87856173126771Q.pdf

[105] S. Szymczak, J. M. Biernacka, H. J. Cordell, O. González-Recio, I. R. König, H. Zhang, Y. V. Sun, O. Gonzalez-Recio, I. R. Konig, H. Zhang, and Y. V. Sun, "Machine learning in genome-wide association studies," Genetic Epidemiology, vol. 33, no. 1, pp. 51-7, 2009. [Online]. Available: https://www.ncbi.nlm.nih.gov/pubmed/19924717 
[106] B. Mieth, M. Kloft, J. A. Rodriguez, S. Sonnenburg, R. Vobruba, C. Morcillo-Suarez, X. Farre, U. M. Marigorta, E. Fehr, T. Dickhaus, G. Blanchard, D. Schunk, A. Navarro, and K. R. Muller, "Combining multiple hypothesis testing with machine learning increases the statistical power of genome-wide association studies," Scientific Reports, vol. 6, p. 36671, 2016. [Online]. Available: https://www.ncbi.nlm.nih.gov/pubmed/27892471

[107] G. S. Long, M. Hussen, J. Dench, and S. Aris-Brosou, "Identifying the genetic determinants of particular phenotypes in microbial genomes with very small training sets," bioRxiv, 2018. [Online]. Available: http://dx.doi.org/10.1101/181222

[108] W. Li and A. Godzik, "Cd-hit: a fast program for clustering and comparing large sets of protein or nucleotide sequences," Bioinformatics, vol. 22, no. 13, pp. 1658-9, 2006. [Online]. Available: https://www.ncbi.nlm.nih.gov/pubmed/16731699

[109] A. M. Kozlov, A. J. Aberer, and A. Stamatakis, "ExaML version 3: a tool for phylogenomic analyses on supercomputers," Bioinformatics, vol. 31, no. 15, pp. 2577-9, 2015. [Online]. Available: https://academic.oup.com/bioinformatics/article-lookup/doi/10.1093/ bioinformatics/btv184

[110] A. Stamatakis, "RAxML version 8: a tool for phylogenetic analysis and post-analysis of large phylogenies," Bioinformatics, vol. 30, no. 9, pp. 1312-3, 2014. [Online]. Available: https://www.ncbi.nlm.nih.gov/pubmed/24451623

[111] M. A. Miller, W. Pfeiffer, and T. Schwartz, "Creating the CIPRES Science Gateway for inference of large phylogenetic trees," 2010 Gateway Computing Environments Workshop (GCE), pp. 1-8, 2010. [Online]. Available: http://www.phylo.org/sub_sections/portal/sc2010_paper.pdf

[112] C. Notredame, D. G. Higgins, and J. Heringa, "T-Coffee: A novel method for fast and accurate multiple sequence alignment," Journal of Microbiology, vol. 302, no. 1, pp. 205-17, 2000. [Online]. Available: https://www.ncbi.nlm.nih.gov/pubmed/10964570

[113] J. R. Dettman, N. Rodrigue, and R. Kassen, "Genome-wide patterns of recombination in the opportunistic human pathogen Pseudomonas aeruginosa." Genome Biology and Evolution, vol. 7, no. 1, pp. 18-34, 2014. [Online]. Available: http://www.ncbi.nlm.nih.gov/pubmed/25480685

[114] J. Tyrrell and M. Callaghan, "Iron acquisition in the cystic fibrosis lung and potential for novel therapeutic strategies." Microbiology, vol. 162, no. 2, pp. 191-205, 2016. [Online]. Available: http://www.ncbi.nlm.nih.gov/pubmed/26643057

[115] F. Minandri, F. Imperi, E. Frangipani, C. Bonchi, D. Visaggio, M. Facchini, P. Pasquali, A. Bragonzi, and P. Visca, "Role of iron uptake systems in Pseudomonas aeruginosa virulence and airway infection." Infection and Immunity, vol. 84, no. 8, pp. 2324-35, 2016. [Online]. Available: http://www.ncbi.nlm.nih.gov/pubmed/27271740

[116] A. Luscher, L. Moynié, P. S. Auguste, D. Bumann, L. Mazza, D. Pletzer, J. H. Naismith, and T. Köhler, "TonB-dependent receptor repertoire of Pseudomonas aeruginosa for uptake of siderophore-drug conjugates." Antimicrobial Agents and Chemotherapy, vol. 62, no. 6, pp. 00 097-18, 2018. [Online]. Available: http://www.ncbi.nlm.nih.gov/pubmed/29555629

[117] J. Dingemans, L. Ye, F. Hildebrand, F. Tontodonati, M. Craggs, F. Bilocq, D. De Vos, A. Crabbé, R. Van Houdt, A. Malfroot, and P. Cornelis, "The deletion of TonB-dependent receptor genes is part of the genome reduction process that occurs during adaptation of Pseudomonas aeruginosa to the cystic fibrosis lung," Pathogens and Disease, vol. 71, no. 1, pp. 26-38, 2014. [Online]. Available: https://academic.oup.com/femspd/article-lookup/doi/10.1111/2049-632X.12170 
[118] C. C. C. R. de Carvalho and P. Fernandes, "Siderophores as "Trojan Horses" tackling multidrug resistance?" Frontiers in Microbiology, vol. 5, p. 290, 2014. [Online]. Available: http://journal.frontiersin.org/article/10.3389/fmicb.2014.00290/abstract

[119] H. Adams, G. Zeder-Lutz, I. Schalk, F. Pattus, and H. Celia, "Interaction of TonB with the outer membrane receptor FpvA of Pseudomonas aeruginosa." Journal of bacteriology, vol. 188, no. 16, pp. 5752-61, 2006. [Online]. Available: http://www.ncbi.nlm.nih.gov/pubmed/16885443

[120] L. M. Faure, S. Garvis, S. De Bentzmann, and S. Bigot, "Characterization of a novel two-partner secretion system implicated in the virulence of Pseudomonas aeruginosa," Microbiology, vol. 160, no. 9, pp. 1940-52, 2014. [Online]. Available: https://www.ncbi.nlm.nih.gov/pubmed/25009238

[121] J. M. Quesada, J. R. Otero-Asman, K. C. Bastiaansen, C. Civantos, and M. A. Llamas, “The activity of the Pseudomonas aeruginosa virulence regulator $\sigma($ VreI $)$ is modulated by the anti- $\sigma$ factor VreR and the transcription factor PhoB." Frontiers in Microbiology, vol. 7, p. 1159, 2016. [Online]. Available: http://www.ncbi.nlm.nih.gov/pubmed/27536271

[122] P. Salunkhe, C. H. M. Smart, J. A. W. Morgan, S. Panagea, M. J. Walshaw, C. A. Hart, R. Geffers, B. Tümmler, and C. Winstanley, "A cystic fibrosis epidemic strain of Pseudomonas aeruginosa displays enhanced virulence and antimicrobial resistance." Journal of Bacteriology, vol. 187, no. 14, pp. 4908-20, 2005. [Online]. Available: http://www.ncbi.nlm.nih.gov/pubmed/15995206

[123] C. Beckmann, M. Brittnacher, R. Ernst, N. Mayer-Hamblett, S. I. Miller, and J. L. Burns, “Use of phage display to identify potential Pseudomonas aeruginosa gene products relevant to early cystic fibrosis airway infections." Infection and Immunity, vol. 73, no. 1, pp. 444-52, 2005. [Online]. Available: http://www.ncbi.nlm.nih.gov/pubmed/15618183

[124] D. Du, X. Wang-Kan, A. Neuberger, H. W. van Veen, K. M. Pos, L. J. V. Piddock, and B. F. Luisi, "Multidrug efflux pumps: structure, function and regulation," Nature Reviews Microbiology, vol. 16, no. 9, pp. 523-39, 2018. [Online]. Available: http://www.nature.com/articles/s41579-018-0048-6

[125] H. Chalhoub, D. Pletzer, H. Weingart, Y. Braun, M. M. Tunney, J. S. Elborn, H. RodriguezVillalobos, P. Plésiat, B. C. Kahl, O. Denis, M. Winterhalter, P. M. Tulkens, and F. Van Bambeke, "Mechanisms of intrinsic resistance and acquired susceptibility of Pseudomonas aeruginosa isolated from cystic fibrosis patients to temocillin, a revived antibiotic." Scientific Reports, vol. 7, p. 40208, 2017. [Online]. Available: http://www.ncbi.nlm.nih.gov/pubmed/28091521

[126] C. Lozano, J. M. Azcona-Gutiérrez, F. Van Bambeke, and Y. Sáenz, "Great phenotypic and genetic variation among successive chronic Pseudomonas aeruginosa from a cystic fibrosis patient," PLoS One, vol. 13, no. 9, 2018. [Online]. Available: http://dx.plos.org/10.1371/journal.pone.0204167

[127] Y. Alguel, D. Lu, N. Quade, S. Sauter, and X. Zhang, "Crystal structure of MexZ, a key repressor responsible for antibiotic resistance in Pseudomonas aeruginosa," Journal of Structural Biology, vol. 172, no. 3, pp. 305-10, 2010. [Online]. Available: https://www.sciencedirect.com/science/article/pii/S1047847710002261?via\%3Dihub

[128] Y. Matsuo, S. Eda, N. Gotoh, E. Yoshihara, and T. Nakae, "MexZ-mediated regulation of mexXY multidrug efflux pump expression in Pseudomonas aeruginosa by binding on the mexZ-mexX intergenic DNA," FEMS Microbiology Letters, vol. 238, no. 1, pp. 23-8, 2004. [Online]. Available: https://academic.oup.com/femsle/article-lookup/doi/10.1111/j. 1574-6968.2004.tb09732.x 
[129] M. H. Prickett, A. R. Hauser, S. A. McColley, J. Cullina, E. Potter, C. Powers, and M. Jain, "Aminoglycoside resistance of Pseudomonas aeruginosa in cystic fibrosis results from convergent evolution in the mexZ gene." Thorax, vol. 72, no. 1, pp. 40-7, 2017. [Online]. Available: http://www.ncbi.nlm.nih.gov/pubmed/27325751

[130] L. Vettoretti, P. Plésiat, C. Muller, F. El Garch, G. Phan, I. Attrée, A. Ducruix, and C. Llanes, "Efflux unbalance in Pseudomonas aeruginosa isolates from cystic fibrosis patients." Antimicrobial Agents and Chemotherapy, vol. 53, no. 5, pp. 1987-97, 2009. [Online]. Available: http://www.ncbi.nlm.nih.gov/pubmed/19258280

[131] J. L. Wylie and E. A. Worobec, "The OprB porin plays a central role in carbohydrate uptake in Pseudomonas aeruginosa." Journal of Bacteriology, vol. 177, no. 11, pp. 3021-6, 1995. [Online]. Available: http://www.ncbi.nlm.nih.gov/pubmed/7768797

[132] T. Mima and H. P. Schweizer, "The BpeAB-OprB efflux pump of Burkholderia pseudomallei $1026 \mathrm{~b}$ does not play a role in quorum sensing, virulence factor production, or extrusion of aminoglycosides but is a broad-spectrum drug efflux system." Antimicrobial Agents and Chemotherapy, vol. 54, no. 8, pp. 3113-20, 2010. [Online]. Available: http://www.ncbi.nlm.nih.gov/pubmed/20498323

[133] N. L. Podnecky, K. A. Rhodes, and H. P. Schweizer, "Efflux pump-mediated drug resistance in Burkholderia." Frontiers in Microbiology, vol. 6, p. 305, 2015. [Online]. Available: http://www.ncbi.nlm.nih.gov/pubmed/25926825

[134] Y. Y. Chan, T. M. C. Tan, Y. M. Ong, and K. L. Chua, “BpeAB-OprB, a multidrug efflux pump in Burkholderia pseudomallei." Antimicrobial Agents and Chemotherapy, vol. 48, no. 4, pp. 1128-35, 2004. [Online]. Available: http://www.ncbi.nlm.nih.gov/pubmed/15047512

[135] D. W. Martin, M. J. Schurr, M. H. Mudd, J. R. Govan, B. W. Holloway, and V. Deretic, "Mechanism of conversion to mucoidy in Pseudomonas aeruginosa infecting cystic fibrosis patients." Proceedings of the National Academy of Sciences of the United States of America, vol. 90, no. 18, pp. 8377-81, 1993. [Online]. Available: http://www.ncbi.nlm.nih.gov/pubmed/8378309

[136] A. Bazire, K. Shioya, E. Soum-Soutéra, E. Bouffartigues, C. Ryder, L. Guentas-Dombrowsky, G. Hémery, I. Linossier, S. Chevalier, D. J. Wozniak, O. Lesouhaitier, and A. Dufour, "The sigma factor AlgU plays a key role in formation of robust biofilms by nonmucoid Pseudomonas aeruginosa." Journal of Bacteriology, vol. 192, no. 12, pp. 3001-10, 2010. [Online]. Available: http://www.ncbi.nlm.nih.gov/pubmed/20348252

[137] M. Shen, H. Zhang, W. Shen, Z. Zou, S. Lu, G. Li, X. He, M. Agnello, W. Shi, F. $\mathrm{Hu}$, and $\mathrm{S}$. Le, "Pseudomonas aeruginosa MutL promotes large chromosomal deletions through non-homologous end joining to prevent bacteriophage predation." Nucleic Acids Research, vol. 46, no. 9, pp. 4505-14, 2018. [Online]. Available: http://www.ncbi.nlm.nih.gov/pubmed/29514250

[138] A. Oliver, F. Baquero, and J. Blazquez, "The mismatch repair system (mutS, mutL and uvrD genes) in Pseudomonas aeruginosa: molecular characterization of naturally occurring mutants," Molecular Microbiology, vol. 43, no. 6, pp. 1641-50, 2002. [Online]. Available: http://doi.wiley.com/10.1046/j.1365-2958.2002.02855.x

[139] J. E. Culbertson and M. D. Toney, "Expression and characterization of PhzE from P. aeruginosa PAO1: aminodeoxyisochorismate synthase involved in pyocyanin and phenazine-1-carboxylate production," Biochimica et Biophysica Acta (BBA) - 
Proteins and Proteomics, vol. 1834, no. 1, pp. 240-6, 2013. [Online]. Available: https://www.sciencedirect.com/science/article/pii/S1570963912002518?via\%3Dihub

[140] C. Kruczek, K. R. Kottapalli, S. Dissanaike, N. Dzvova, J. A. Griswold, J. A. Colmer-Hamood, and A. N. Hamood, "Major transcriptome changes accompany the growth of Pseudomonas aeruginosa in blood injuries." PLoS One, vol. 11, no. 3, 2016. [Online]. Available: http://www.ncbi.nlm.nih.gov/pubmed/26933952

[141] M. Carlsson, S. Shukla, A. C. Petersson, M. Segelmark, and T. Hellmark, "Pseudomonas aeruginosa in cystic fibrosis: Pyocyanin negative strains are associated with BPI-ANCA and progressive lung disease," Journal of Cystic Fibrosis, vol. 10, no. 4, pp. 265-71, 2011. [Online]. Available: https://www.sciencedirect.com/science/article/pii/S1569199311000440? via\%3Dihub

[142] L. Wang, P. Jia, R. D. Wolfinger, X. Chen, and Z. Zhao, "Gene set analysis of genome-wide association studies: methodological issues and perspectives." Genomics, vol. 98, no. 1, pp. 1-8, 2011. [Online]. Available: http://www.ncbi.nlm.nih.gov/pubmed/21565265

[143] J. Zhou, D. Hao, X. Wang, T. Liu, C. He, F. Xie, Y. Sun, and J. Zhang, "An important role of a "probable ATP-binding component of ABC transporter" during the process of Pseudomonas aeruginosa resistance to fluoroquinolone," Proteomics, vol. 6, no. 8, pp. 2495-503, 2006. [Online]. Available: http://www.ncbi.nlm.nih.gov/pubmed/16526085

[144] N. Gliese, V. Khodaverdi, and H. Görisch, "The PQQ biosynthetic operons and their transcriptional regulation in Pseudomonas aeruginosa," Archives of Microbiology, vol. 192, no. 1, pp. 1-14, 2010. [Online]. Available: http://link.springer.com/10.1007/s00203-009-0523-6

[145] Q. Wei, T. Ran, C. Ma, J. He, D. Xu, and W. Wang, "Crystal structure and function of PqqF protein in the pyrroloquinoline quinone biosynthetic pathway." Journal of Biological Chemistry, vol. 291, no. 30, pp. 15575-87, 2016. [Online]. Available: http://www.ncbi.nlm.nih.gov/pubmed/27231346

[146] D. Kocíncová, Y. Hao, E. Vinogradov, and J. S. Lam, "Evidence that WapB is a 1,2-glucosyltransferase of Pseudomonas aeruginosa involved in Lipopolysaccharide outer core biosynthesis." Journal of Bacteriology, vol. 193, no. 11, pp. 2708-16, 2011. [Online]. Available: http://www.ncbi.nlm.nih.gov/pubmed/21441506

[147] D. Kocíncová and J. S. Lam, "A deletion in the wapB promoter in many serotypes of Pseudomonas aeruginosa accounts for the lack of a terminal glucose residue in the core oligosaccharide and resistance to killing by R3-pyocin," Molecular Microbiology, vol. 89, no. 3, pp. 464-78, 2013. [Online]. Available: http://www.ncbi.nlm.nih.gov/pubmed/23750877

[148] G. B. Pier, "Pseudomonas aeruginosa lipopolysaccharide: a major virulence factor, initiator of inflammation and target for effective immunity." International Journal of Medical Microbiology, vol. 297, no. 5, pp. 277-95, 2007. [Online]. Available: http://www.ncbi.nlm.nih.gov/pubmed/17466590

[149] K. Schreiber, R. Krieger, B. Benkert, M. Eschbach, H. Arai, M. Schobert, and D. Jahn, "The anaerobic regulatory network required for Pseudomonas aeruginosa nitrate respiration." Journal of Bacteriology, vol. 189, no. 11, pp. 4310-4, 2007. [Online]. Available: http://www.ncbi.nlm.nih.gov/pubmed/17400734

[150] J. F. Ghiglione, L. Philippot, P. Normand, R. Lensi, and P. Potier, “Disruption of narG, the gene encoding the catalytic subunit of respiratory nitrate reductase, also affects nitrite respiration in Pseudomonas fluorescens YT101." Journal of Bacteriology, vol. 181, no. 16, pp. 5099-102, 1999. [Online]. Available: http://www.ncbi.nlm.nih.gov/pubmed/10438786 
[151] G. McCaughey, D. F. Gilpin, T. Schneiders, L. R. Hoffman, M. McKevitt, J. S. Elborn, and M. M. Tunney, "Fosfomycin and tobramycin in combination downregulate nitrate reductase genes narG and narH, resulting in increased activity against Pseudomonas aeruginosa under anaerobic conditions." Antimicrobial Agents and Chemotherapy, vol. 57, no. 11, pp. 5406-14, 2013. [Online]. Available: http://www.ncbi.nlm.nih.gov/pubmed/23959314

[152] Z. Xu, X. Fang, T. K. Wood, and Z. J. Huang, “A systems-level approach for investigating Pseudomonas aeruginosa biofilm formation," PLoS One, vol. 8, no. 2, 2013. [Online]. Available: http://www.ncbi.nlm.nih.gov/pubmed/23451140

[153] B. Thöny, D. S. Hwang, L. Fradkin, and A. Kornberg, "iciA, an Escherichia coli gene encoding a specific inhibitor of chromosomal initiation of replication in vitro." Proceedings of the National Academy of Sciences of the United States of America, vol. 88, no. 10, pp. 4066-70, 1991. [Online]. Available: http://www.ncbi.nlm.nih.gov/pubmed/2034653

[154] H. Hu, H. Zhang, Z. Gao, D. Wang, G. Liu, J. Xu, K. Lan, and Y. Dong, "Structure of the type VI secretion phospholipase effector Tle1 provides insight into its hydrolysis and membrane targeting," Acta Crystallographica Section D Biological Crystallography, vol. 70, no. 8, pp. 2175-85, 2014. [Online]. Available: http://www.ncbi.nlm.nih.gov/pubmed/25084336

[155] L. Liu, M. Ye, X. Li, J. Li, Z. Deng, Y.-F. Yao, and H.-Y. Ou, “Identification and characterization of an antibacterial type VI secretion system in the carbapenem-resistant strain Klebsiella pneumoniae HS11286." Frontiers in Cellular and Infection Microbiology, vol. 7, p. 442, 2017. [Online]. Available: http://www.ncbi.nlm.nih.gov/pubmed/29085808

[156] M. R. Davis, A. Muszyński, I. V. Lollett, C. L. Pritchett, R. W. Carlson, and J. B. Goldberg, "Identification of the mutation responsible for the temperature-sensitive lipopolysaccharide O-antigen defect in the Pseudomonas aeruginosa cystic fibrosis isolate 2192," Journal of Bacteriology, vol. 195, no. 7, pp. 1504-14, 2013. [Online]. Available: http://www.ncbi.nlm.nih.gov/pubmed/23354750

[157] L. L. Burrows, D. F. Charter, and J. S. Lam, "Molecular characterization of the Pseudomonas aeruginosa serotype O5 (PAO1) B-band lipopolysaccharide gene cluster," Molecular Microbiology, vol. 22, no. 3, pp. 481-95, 1996. [Online]. Available: http://doi.wiley.com/10.1046/j.1365-2958.1996.1351503.x

[158] M. A. Oberhardt, J. Puchałka, K. E. Fryer, V. A. P. Martins dos Santos, and J. A. Papin, "Genome-scale metabolic network analysis of the opportunistic pathogen Pseudomonas aeruginosa PAO1." Journal of Bacteriology, vol. 190, no. 8, pp. 2790-803, 2008. [Online]. Available: http://www.ncbi.nlm.nih.gov/pubmed/18192387

[159] B. Ghysels, U. Ochsner, U. Möllman, L. Heinisch, M. Vasil, P. Cornelis, and S. Matthijs, "The Pseudomonas aeruginosa pirA gene encodes a second receptor for ferrienterobactin and synthetic catecholate analogues," FEMS Microbiology Letters, vol. 246, no. 2, pp. 167-74, 2005. [Online]. Available: https://academic.oup.com/femsle/article-lookup/doi/10.1016/j. femsle.2005.04.010

[160] L. Moynié, A. Luscher, D. Rolo, D. Pletzer, A. Tortajada, H. Weingart, Y. Braun, M. G. P. Page, J. H. Naismith, and T. Köhler, "Structure and function of the PiuA and PirA siderophore-drug receptors from Pseudomonas aeruginosa and Acinetobacter baumannii." Antimicrobial Agents and Chemotherapy, vol. 61, no. 4, 2017. [Online]. Available: http:/ / www.ncbi.nlm.nih.gov/pubmed/28137795 
[161] J. Botelho, F. Grosso, and L. Peixe, "Unravelling the genome of a Pseudomonas aeruginosa isolate belonging to the high-risk clone ST235 reveals an integrative conjugative element housing a blaGES-6 carbapenemase," Journal of Antimicrobial Chemotherapy, vol. 73, no. 1, pp. 77-83, 2018. [Online]. Available: http://academic.oup.com/jac/article/73/1/77/4339348

[162] J. Tremblay and E. Déziel, "Gene expression in Pseudomonas aeruginosa swarming motility." BMC Genomics, vol. 11, p. 587, 2010. [Online]. Available: http://www.ncbi.nlm.nih.gov/ pubmed/20961425

[163] D. Portik and J. Wiens, "SuperCRUNCH: A toolkit for creating and manipulating supermatrices and other large phylogenetic datasets," bioRxiv, p. 538728, 2019. [Online]. Available: https://www.biorxiv.org/content/10.1101/538728v1

[164] A. Bateman, M. T. G. Holden, and C. Yeats, “The G5 domain: a potential N-acetylglucosamine recognition domain involved in biofilm formation," Bioinformatics, vol. 21, no. 8, pp. 1301-3, 2005. [Online]. Available: https://academic.oup.com/bioinformatics/article-lookup/doi/10. 1093/bioinformatics/bti206

[165] T. L. Lindsey, J. M. Hagins, P. A. Sokol, and L. A. Silo-Suh, "Virulence determinants from a cystic fibrosis isolate of Pseudomonas aeruginosa include isocitrate lyase," Microbiology, vol. 154, no. 6, pp. 1616-27, 2008. [Online]. Available: http://www.ncbi.nlm.nih.gov/pubmed/18524916

[166] P. J. Intile, G. J. Balzer, M. C. Wolfgang, and T. L. Yahr, “The RNA helicase DeaD stimulates ExsA translation to promote expression of the Pseudomonas aeruginosa type III secretion system." Journal of Bacteriology, vol. 197, no. 16, pp. 2664-74, 2015. [Online]. Available: http://www.ncbi.nlm.nih.gov/pubmed/26055113

[167] J.-F. Dubern, C. Cigana, M. De Simone, J. Lazenby, M. Juhas, S. Schwager, I. Bianconi, G. Döring, L. Eberl, P. Williams, A. Bragonzi, and M. Cámara, “Integrated whole-genome screening for Pseudomonas aeruginosa virulence genes using multiple disease models reveals that pathogenicity is host specific." Environmental Microbiology, vol. 17, no. 11, pp. 4379-93, 2015. [Online]. Available: http://www.ncbi.nlm.nih.gov/pubmed/25845292

[168] J. Shuman, T. X. Giles, L. Carroll, K. Tabata, A. Powers, S.-J. Suh, and L. Silo-Suh, "Transcriptome analysis of a Pseudomonas aeruginosa sn-glycerol-3-phosphate dehydrogenase mutant reveals a disruption in bioenergetics," Microbiology, vol. 164, no. 4, pp. 551-62, 2018. [Online]. Available: http://www.ncbi.nlm.nih.gov/pubmed/29533746

[169] J. Dingemans, P. Monsieurs, S.-H. Yu, A. Crabbé, K. U. Förstner, A. Malfroot, P. Cornelis, and R. Van Houdt, "Effect of shear stress on Pseudomonas aeruginosa isolated from the cystic fibrosis lung." mBio, vol. 7, no. 4, 2016. [Online]. Available: http://www.ncbi.nlm.nih.gov/pubmed/27486191

[170] H. S. Girgis, K. Harris, and S. Tavazoie, "Large mutational target size for rapid emergence of bacterial persistence." Proceedings of the National Academy of Sciences of the United States of America, vol. 109, no. 31, pp. 12740-5, 2012. [Online]. Available: http://www.ncbi.nlm.nih.gov/pubmed/22802628

[171] C. Winstanley, S. O'Brien, and M. A. Brockhurst, "Pseudomonas aeruginosa evolutionary adaptation and diversification in cystic fibrosis chronic lung infections." Trends in Microbiology, vol. 24, no. 5, pp. 327-37, 2016. [Online]. Available: http://www.ncbi.nlm.nih.gov/pubmed/26946977 
[172] F. H. Damron and H. D. Yu, "Pseudomonas aeruginosa MucD regulates the alginate pathway through activation of MucA degradation via MucP proteolytic activity," Journal of Bacteriology, vol. 193, no. 1, pp. 286-91, 2011. [Online]. Available: https://jb.asm.org/content/193/1/286

[173] L. F. Wood and D. E. Ohman, "Independent regulation of MucD, an HtrA-like protease in Pseudomonas aeruginosa, and the role of its proteolytic motif in alginate gene regulation." Journal of Bacteriology, vol. 188, no. 8, pp. 3134-7, 2006. [Online]. Available: http://www.ncbi.nlm.nih.gov/pubmed/16585775

[174] P. Tielen, N. Rosin, A.-K. Meyer, K. Dohnt, I. Haddad, L. Jänsch, J. Klein, M. Narten, C. Pommerenke, M. Scheer, M. Schobert, D. Schomburg, B. Thielen, and D. Jahn, "Regulatory and metabolic networks for the adaptation of Pseudomonas aeruginosa biofilms to urinary tract-like conditions," PLoS One, vol. 8, no. 8, 2013. [Online]. Available: https://dx.plos.org/10.1371/journal.pone.0071845

[175] K. Remans, K. Vercammen, J. Bodilis, and P. Cornelis, “Genome-wide analysis and literaturebased survey of lipoproteins in Pseudomonas aeruginosa," Microbiology, vol. 156, no. 9, pp. 2597-607, 2010. [Online]. Available: http://www.ncbi.nlm.nih.gov/pubmed/20616104

[176] G. Sigurdsson, R. M. T. Fleming, A. Heinken, and I. Thiele, “A systems biology approach to drug targets in Pseudomonas aeruginosa biofilm." PLoS One, vol. 7, no. 4, 2012. [Online]. Available: http://www.ncbi.nlm.nih.gov/pubmed/22523548

[177] C. J. Harmer, M. Wynn, R. Pinto, S. Cordwell, B. R. Rose, C. Harbour, J. A. Triccas, and J. Manos, "Homogentisate 1-2-dioxygenase downregulation in the chronic persistence of Pseudomonas aeruginosa Australian Epidemic Strain-1 in the CF lung." PLoS One, vol. 10, no. 8, 2015. [Online]. Available: http://www.ncbi.nlm.nih.gov/pubmed/26252386

[178] B. R. Lundgren, Z. Sarwar, A. Pinto, J. G. Ganley, and C. T. Nomura, "Ethanolamine catabolism in Pseudomonas aeruginosa PAO1 is regulated by the enhancer-binding protein EatR (PA4021) and the alternative sigma factor RpoN." Journal of Bacteriology, vol. 198, no. 17, pp. 2318-29, 2016. [Online]. Available: http://www.ncbi.nlm.nih.gov/pubmed/27325678

[179] K. Taniyama, H. Itoh, A. Takuwa, Y. Sasaki, S. Yajima, M. Toyofuku, N. Nomura, and N. Takaya, "Group X aldehyde dehydrogenases of Pseudomonas aeruginosa PAO1 degrade hydrazones." Journal of Bacteriology, vol. 194, no. 6, pp. 1447-56, 2012. [Online]. Available: http:/ / www.ncbi.nlm.nih.gov/pubmed/22267508

[180] E. V. A. Sylvester, P. Bentzen, I. R. Bradbury, M. Clément, J. Pearce, J. Horne, and R. G. Beiko, "Applications of random forest feature selection for fine-scale genetic population assignment." Evolutionary Applications, vol. 11, no. 2, pp. 153-65, 2018. [Online]. Available: http://www.ncbi.nlm.nih.gov/pubmed/29387152

[181] M. T. Uddin and M. A. Uddiny, "A guided random forest based feature selection approach for activity recognition," in 2015 International Conference on Electrical Engineering and Information Communication Technology (ICEEICT). IEEE, 2015, pp. 1-6. [Online]. Available: http://ieeexplore.ieee.org/document/7307376/

[182] C. Hoboth, R. Hoffmann, A. Eichner, C. Henke, S. Schmoldt, A. Imhof, J. Heesemann, and M. Hogardt, "Dynamics of adaptive microevolution of hypermutable Pseudomonas aeruginosa during chronic pulmonary infection in patients with cystic fibrosis," Journal of Infectious Diseases, vol. 200, no. 1, pp. 118-30, 2009. [Online]. Available: https://academic.oup.com/jid/article-lookup/doi/10.1086/599360 


\section{A. BACKGROUND INFORMATION}

\section{A.1. Genes/proteins associated with CF-phenotype of PA}

Table 17: Genes and proteins important for the CF-phenotype as identified in several review papers.

\begin{tabular}{|c|c|c|}
\hline RefSeq & $\begin{array}{l}\text { Gene } \\
\text { name }\end{array}$ & Product name \\
\hline NP_251075.1 & pvdQ & 3-oxo-C12-homoserine lactone acylase PvdQ \\
\hline NP_251852.1 & rpsA & 30S ribosomal protein $\mathrm{S} 1$ \\
\hline NP_253342.1 & cupE6 & Adhesin-like protein CupE6 \\
\hline NP_252771.1 & cupB5 & adhesive protein CupB5 \\
\hline NP_252235.1 & $\operatorname{alg} G$ & alginate-c5-mannuronan-epimerase $A \lg G$ \\
\hline NP_253887.1 & amgR & AmgR \\
\hline NP_253886.1 & amgS & AmgS \\
\hline NP_253911.1 & pepP & aminopeptidase $\mathrm{P}$ \\
\hline NP_249454.1 & mucA & anti-sigma factor MucA \\
\hline NP_249649.1 & oprD & outer membrane porin OprD precursor \\
\hline NP_253212.1 & ampD & beta-lactamase expression regulator $\mathrm{AmpD}$ \\
\hline NP_252799.1 & ampC & beta-lactamase precursor \\
\hline NP_253057.1 & bifÄ & BifA \\
\hline NP_250819.1 & cupA2 & chaperone CupA2 \\
\hline NP_250822.1 & cupA5 & chaperone CupA5 \\
\hline NP_252774.1 & cupB2 & chaperone CupB2 \\
\hline NP_252772.1 & cupB4 & chaperone CupB4 \\
\hline NP_249684.1 & cupC2 & chaperone CupC2 \\
\hline NP_249104.1 & chpA & component of chemotactic signal transduction system \\
\hline NP_253389.1 & & conserved hypothetical protein \\
\hline NP_253649.1 & & conserved hypothetical protein \\
\hline NP_253820.1 & & conserved hypothetical protein \\
\hline NP_253991.1 & $\operatorname{dad} A$ & D-amino acid dehydrogenase, small subunit \\
\hline NP_253703.1 & aceF & dihydrolipoamide acetyltransferase \\
\hline NP_251858.1 & gyrA & DNA gyrase subunit A \\
\hline NP_064724.1 & gyrB & DNA gyrase subunit B \\
\hline NP_253633.1 & mutL & DNA mismatch repair protein MutL \\
\hline NP_252310.1 & mutS & DNA mismatch repair protein MutS \\
\hline NP_252956.1 & fusA1 & elongation factor $\mathrm{G}$ \\
\hline NP_250405.1 & exsD & ExsD \\
\hline NP_250818.1 & cupA1 & fimbrial subunit CupA1 \\
\hline NP_250821.1 & cupA4 & fimbrial subunit CupA4 \\
\hline NP_252770.1 & cupB6 & fimbrial subunit CupB6 \\
\hline NP_249683.1 & cupC1 & fimbrial subunit CupC1 \\
\hline NP_253341.1 & cupE5 & Fimbrial usher protein CupE5 \\
\hline NP_252907.1 & phzS & flavin-containing monooxygenase \\
\hline NP_253398.1 & phuR & $\begin{array}{l}\text { Heme/Hemoglobin uptake outer membrane receptor PhuR } \\
\text { precursor }\end{array}$ \\
\hline NP_252396.1 & wspB & hypothetical protein \\
\hline NP_252394.1 & wspD & hypothetical protein \\
\hline NP_251783.1 & & hypothetical protein \\
\hline NP_253648.1 & & hypothetical protein \\
\hline
\end{tabular}




\begin{tabular}{|c|c|c|}
\hline RefSeq & $\begin{array}{l}\text { Gene } \\
\text { name }\end{array}$ & Product name \\
\hline NP_251103.1 & pvdH & $\begin{array}{l}\text { L-2,4-diaminobutyrate:2-ketoglutarate 4-aminotransferase, } \\
\text { PvdH }\end{array}$ \\
\hline NP_249004.1 & & L-cysteine transporter of ABC system YecS \\
\hline NP_251076.1 & pvdA & L-ornithine N5-oxygenase \\
\hline NP_253885.1 & & LD-carboxypeptidase \\
\hline NP_250509.1 & cadA & lysine decarboxylase \\
\hline NP_249118.1 & oprM & multidrug efflux outer membrane protein OprM precursor \\
\hline NP_250503.1 & mltD & membrane-bound lytic murein transglycosylase D precursor \\
\hline NP_249103.1 & pilK & methyltransferase PilK \\
\hline NP_251181.1 & mexS & MexS \\
\hline NP_250710.1 & $\operatorname{mexZ}$ & MexZ \\
\hline NP_253291.1 & morA & motility regulator \\
\hline NP_249115.1 & $\operatorname{mexR}$ & multidrug resistance operon repressor MexR \\
\hline NP_253846.1 & & multidrug resistance protein \\
\hline NP_252264.1 & nalD & NalD \\
\hline NP_251543.1 & oprI & Outer membrane lipoprotein OprI precursor \\
\hline NP_251754.1 & pelA & PelA \\
\hline NP_253108.1 & ftsI & penicillin-binding protein 3 \\
\hline NP_252901.1 & phzC1 & phenazine biosynthesis protein $\mathrm{PhzC}$ \\
\hline NP_250592.1 & phzC2 & phenazine biosynthesis protein $\mathrm{PhzC}$ \\
\hline NP_252902.1 & phzD1 & phenazine biosynthesis protein PhzD \\
\hline NP_250593.1 & phzD2 & phenazine biosynthesis protein PhzD \\
\hline NP_252903.1 & phzE1 & phenazine biosynthesis protein PhzE \\
\hline NP_250594.1 & phzE2 & phenazine biosynthesis protein PhzE \\
\hline NP_253340.1 & cupE4 & Pilin assembly chaperone CupE4 \\
\hline NP_253337.1 & cupE1 & Pilin subunit CupE1 \\
\hline NP_253338.1 & cupE2 & Pilin subunit CupE2 \\
\hline NP_253339.1 & cupE3 & Pilin subunit CupE3 \\
\hline NP_253464.1 & pmrA & $\begin{array}{l}\text { PmrA: two-component regulator system response regulator } \\
\text { PmrA }\end{array}$ \\
\hline NP_253747.1 & phaF & polyhydroxyalkanoate synthesis protein PhaF \\
\hline NP_248741.1 & phzH & potential phenazine-modifying enzyme \\
\hline NP_253492.1 & & POTRA-like domain-containing usher, CupB3 \\
\hline NP_252393.1 & wspE & probable chemotaxis sensor/effector fusion protein \\
\hline NP_252397.1 & wspA & probable chemotaxis transducer \\
\hline NP_253238.1 & & probable D-amino acid oxidase \\
\hline NP_252775.1 & cupB1 & probable fimbrial subunit CupB1 \\
\hline NP_252392.1 & wspF & probable methylesterase \\
\hline NP_252899.1 & phzA1 & probable phenazine biosynthesis protein \\
\hline NP_250590.1 & phzA2 & probable phenazine biosynthesis protein \\
\hline NP_252900.1 & phzB1 & probable phenazine biosynthesis protein \\
\hline NP_250591.1 & phzB2 & probable phenazine biosynthesis protein \\
\hline NP_252904.1 & phzF1 & probable phenazine biosynthesis protein \\
\hline NP_250595.1 & phzF2 & probable phenazine biosynthesis protein \\
\hline NP_252898.1 & phzM & probable phenazine-specific methyltransferase \\
\hline NP_252395.1 & wspC & probable protein methyltransferase \\
\hline NP_252906.1 & phzG1 & probable pyridoxamine 5 -phosphate oxidase \\
\hline NP_252905.1 & phzG2 & probable pyridoxamine $5^{\prime}$-phosphate oxidase \\
\hline
\end{tabular}




\begin{tabular}{|c|c|c|}
\hline RefSeq & $\begin{array}{l}\text { Gene } \\
\text { name }\end{array}$ & Product name \\
\hline NP_249226.1 & & probable transcriptional regulator \\
\hline NP_250921.1 & pslA & PslA \\
\hline NP_251115.1 & pvdG & PvdG \\
\hline NP_251090.2 & pvdJ & PvdJ \\
\hline NP_251114.1 & pvdL & PvdL \\
\hline NP_251084.1 & pvdN & PvdN \\
\hline NP_251085.1 & pvdO & PvdO \\
\hline NP_251082.1 & pvdP & PvdP \\
\hline NP_251079.1 & pvdR & PvdR \\
\hline NP_251080.1 & pvdT & PvdT \\
\hline NP_251087.1 & pvdE & pyoverdine biosynthesis protein PvdE \\
\hline NP_251089.1 & pvdD & pyoverdine synthetase D \\
\hline NP_251086.1 & pvdF & pyoverdine synthetase $\mathrm{F}$ \\
\hline NP_253702.1 & aceE & pyruvate dehydrogenase \\
\hline NP_250589.1 & qscR & quorum-sensing control repressor \\
\hline NP_249552.1 & $\mathrm{rbdA}$ & $\mathrm{RbDA}$ \\
\hline NP_249116.1 & $\operatorname{mexA}$ & multidrug efflux membrane fusion protein MexA precursor \\
\hline NP_249117.1 & $\operatorname{mexB}$ & multidrug efflux transporter MexB \\
\hline NP_253543.1 & retS & $\begin{array}{l}\text { RetS (Regulator of Exopolysaccharide and Type III Secre- } \\
\text { tion) }\end{array}$ \\
\hline NP_253152.1 & rpoN & RNA polymerase sigma-54 factor \\
\hline NP_249619.1 & gacS & sensor/response regulator hybrid \\
\hline NP_249749.1 & shaE & ShaE \\
\hline NP_249453.1 & $\operatorname{alg} U$ & sigma factor AlgU \\
\hline NP_251116.1 & pvdS & sigma factor PvdS \\
\hline NP_253654.1 & parE & topoisomerase IV subunit B \\
\hline NP_252798.1 & ampR & transcriptional regulator $\mathrm{AmpR}$ \\
\hline NP_250121.1 & lasR & transcriptional regulator LasR \\
\hline NP_251182.1 & mexT & transcriptional regulator MexT \\
\hline NP_253290.1 & nfxB & transcriptional regulator $\mathrm{Nf} \times \mathrm{B}$ \\
\hline NP_249099.1 & pilG & twitching motility protein PilG \\
\hline NP_249100.1 & pilH & twitching motility protein PilH \\
\hline NP_249101.1 & pill & twitching motility protein Pill \\
\hline NP_249102.1 & pilJ & twitching motility protein PilJ \\
\hline NP_249086.1 & pilT & twitching motility protein PilT \\
\hline NP_249087.1 & pilU & twitching motility protein PilU \\
\hline NP_253237.1 & pilR & two-component response regulator PilR \\
\hline NP_253236.1 & pils & two-component sensor PilS \\
\hline NP_253727.1 & pilQ & type 4 fimbrial biogenesis protein PilQ precursor \\
\hline NP_253216.1 & pilB & type 4 fimbrial biogenesis protein PilB \\
\hline NP_253246.1 & pilE & type 4 fimbrial biogenesis protein PilE \\
\hline NP_252494.1 & pilF & type 4 fimbrial biogenesis protein PilF \\
\hline NP_253731.1 & pilM & type 4 fimbrial biogenesis protein PilM \\
\hline NP_253730.1 & pilN & type 4 fimbrial biogenesis protein PilN \\
\hline NP_253729.1 & pilO & type 4 fimbrial biogenesis protein PilO \\
\hline NP_253728.1 & pilP & type 4 fimbrial biogenesis protein PilP \\
\hline NP_253241.1 & pilV & type 4 fimbrial biogenesis protein PilV \\
\hline NP_253242.1 & pilW & type 4 fimbrial biogenesis protein PilW \\
\hline
\end{tabular}




\begin{tabular}{lll}
\hline RefSeq & $\begin{array}{l}\text { Gene } \\
\text { name }\end{array}$ & Product name \\
\hline NP_253243.1 & pilX & type 4 fimbrial biogenesis protein PilX \\
NP_253244.1 & pilY1 & type 4 fimbrial biogenesis protein PilY1 \\
NP_253245.1 & pilY2 & type 4 fimbrial biogenesis protein PilY2 \\
NP_251650.1 & pilZ & type 4 fimbrial biogenesis protein PilZ \\
NP_253215.1 & pilA & type 4 fimbrial precursor PilA \\
NP_253218.1 & pilD & type 4 prepilin peptidase PilD \\
NP_253910.1 & ubiH & ubiH protein \\
NP_252709.1 & mpl & UDP-N-acetylmuramate: L-alanyl-gamma-D-glutamyl- \\
& & meso-diaminopimelate ligase \\
NP_250820.1 & cupA3 & usher CupA3 \\
NP_252773.1 & cupB3 & usher CupB3 \\
NP_249685.1 & cupC3 & usher CupC3 \\
NP_248781.1 & vgrG1 & VgrG1 \\
NP_250202.1 & vgrG2a & VgrG2a \\
NP_248953.1 & vgrG2b & VgrG2b \\
NP_251063.1 & vgrG3 & VgrG3 \\
NP_251375.1 & vgrG4 & VgrG4 \\
NP_251984.1 & vgrG4a & VgrG4a \\
NP_252176.1 & vgrG4b & VgrG4b \\
NP_253777.1 & vgrG5 & VgrG5 \\
NP_253953.1 & vgrG6 & VgrG6 \\
NP_252391.1 & wspR & WspR \\
& mutL & DNA mismatch repair protein MutL (pseudogene) \\
& mutS & DNA mismatch repair protein MutS (pseudogene) \\
\hline & &
\end{tabular}

\section{B. Results}

\section{B.1. Dataset 1 PLINK results}

Table 18: Genes identified with PLINK from dataset 1 , gene presence/absence. Genes with $p$ values less than or equal to $2.54 \times 10^{-10}$ are reported.

\begin{tabular}{lllllr}
\hline Rank & Accession & $\begin{array}{c}\text { Gene } \\
\text { name }\end{array}$ & Protein name & p value & $\begin{array}{c}\text { Odds } \\
\text { ratio }\end{array}$ \\
\hline 1 & NP_248888.1 & tonB2 & TonB2 & $3.09 \times 10^{-33}$ & 41.62 \\
2 & & & $\begin{array}{l}\text { non-ribosomal peptide } \\
\text { synthetase (pseudogene) }\end{array}$ & $1.32 \times 10^{-28}$ & 27.22 \\
& & & & \\
3 & & ffs & $1.70 \times 10^{-26}$ & 31.17 \\
4 & NP_251800.1 & & PA3110 & $4.36 \times 10^{-25}$ & 15.85 \\
5 & NP_064723.1 & recF & recF & $1.66 \times 10^{-20}$ & 125.1 \\
6 & & & DNA-binding response & $2.48 \times 10^{-20}$ & 2077 \\
& & & regulator (pseudogene) & & \\
7 & & nan & $3.14 \times 10^{-20}$ & 22.75 \\
8 & NP_249603.1 & PA0912 & $8.84 \times 10^{-20}$ & 712.5 \\
9 & & nan & $1.07 \times 10^{-19}$ & 9.60 \\
10 & NP_253159.1 & PA4469 & $2.25 \times 10^{-19}$ & NA \\
11 & NP_253319.1 & PA4629 & $6.21 \times 10^{-19}$ & NA \\
\hline
\end{tabular}




\begin{tabular}{|c|c|c|c|c|c|}
\hline Rank & Accession & $\begin{array}{l}\text { Gene } \\
\text { name }\end{array}$ & Protein name & $\mathrm{p}$ value & $\begin{array}{l}\text { Odds } \\
\text { ratio }\end{array}$ \\
\hline 12 & NP_249620.1 & & $\begin{array}{l}\text { two-component response } \\
\text { regulator }\end{array}$ & $1.63 \times 10^{-18}$ & 20.04 \\
\hline 13 & NP_253284.1 & & $\begin{array}{l}\text { PA4594 probable ATP-binding } \\
\text { component of ABC transporter }\end{array}$ & $2.13 \times 10^{-18}$ & 2023 \\
\hline 14 & NP_250230.1 & & 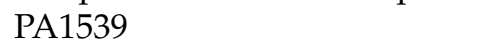 & $2.39 \times 10^{-18}$ & 486.5 \\
\hline 15 & & & nan & $2.89 \times 10^{-18}$ & 2093 \\
\hline 16 & NP_249381.1 & $p d t A$ & $\begin{array}{l}\text { phosphate depletion regulated } \\
\text { TPS partner A, PdtA }\end{array}$ & $4.08 \times 10^{-18}$ & 22.15 \\
\hline 17 & NP_249374.1 & $h x c y$ & $\mathrm{HxcY}$ & $6.46 \times 10^{-18}$ & 37.15 \\
\hline 18 & NP_249929.1 & & $\begin{array}{l}\text { PA1238 probable outer } \\
\text { membrane component of } \\
\text { multidrug efflux pump }\end{array}$ & $6.70 \times 10^{-18}$ & 24.35 \\
\hline 19 & NP_250420.1 & & PA1729 & $1.33 \times 10^{-17}$ & 501.3 \\
\hline 20 & NP_249865.1 & napA & $\begin{array}{l}\text { periplasmic nitrate reductase } \\
\text { protein NapA }\end{array}$ & $2.62 \times 10^{-17}$ & 28.32 \\
\hline 21 & & & $\begin{array}{l}\text { hypothetical protein } \\
\text { (pseudogene) }\end{array}$ & $3.34 \times 10^{-17}$ & 171.8 \\
\hline 22 & NP_250018.1 & & PA1327 probable protease & $5.99 \times 10^{-17}$ & 253.1 \\
\hline 23 & & & $\begin{array}{l}\text { ABC transporter ATP-binding } \\
\text { protein (pseudogene) }\end{array}$ & $6.07 \times 10^{-17}$ & NA \\
\hline 24 & NP_250710.1 & mexz & MexZ & $6.61 \times 10^{-16}$ & 9.85 \\
\hline 25 & NP_251876.1 & $o p r B$ & $\begin{array}{l}\text { Glucose/carbohydrate outer } \\
\text { membrane porin OprB } \\
\text { precursor }\end{array}$ & $1.38 \times 10^{-15}$ & 6.32 \\
\hline 26 & & & nan & $1.86 \times 10^{-15}$ & 31.26 \\
\hline 27 & NP_253331.1 & & PA4642 & $2.70 \times 10^{-15}$ & 14.69 \\
\hline 28 & & & nan & $5.63 \times 10^{-15}$ & 27.87 \\
\hline 29 & & & $\begin{array}{l}\text { sulfonate } \mathrm{ABC} \text { transporter } \\
\text { substrate-binding protein } \\
\text { (pseudogene) }\end{array}$ & $5.72 \times 10^{-15}$ & 21.64 \\
\hline 30 & NP_249454.1 & mисA & mucA & $5.73 \times 10^{-15}$ & 23.65 \\
\hline 31 & & & mutL (pseudogene) & $7.61 \times 10^{-15}$ & 20.65 \\
\hline 32 & & & $\begin{array}{l}\text { precorrin-3B } \\
\text { C(17)-methyltransferase } \\
\text { (pseudogene) }\end{array}$ & $2.76 \times 10^{-14}$ & 22.18 \\
\hline 33 & NP_249117.1 & $\operatorname{mex} B$ & $\begin{array}{l}\text { Resistance-Nodulation-Cell } \\
\text { Division (RND) multidrug } \\
\text { efflux transporter MexB }\end{array}$ & $3.61 \times 10^{-14}$ & 18.67 \\
\hline 34 & & & $\begin{array}{l}\text { cysteine dioxygenase } \\
\text { (pseudogene) }\end{array}$ & $6.98 \times 10^{-14}$ & 20.06 \\
\hline 35 & NP_253474.1 & & $\begin{array}{l}\text { PA4786 probable short-chain } \\
\text { dehydrogenase }\end{array}$ & $7.23 \times 10^{-14}$ & 28.39 \\
\hline 36 & NP_250376.1 & mas $A$ & enolase-phosphatase E-1 & $7.61 \times 10^{-14}$ & 31.37 \\
\hline 37 & NP_253724.1 & & PA5037 & $1.05 \times 10^{-13}$ & 33.52 \\
\hline 38 & & & $\begin{array}{l}\text { 3-oxoacyl-ACP reductase } \\
\text { (pseudogene) }\end{array}$ & $3.30 \times 10^{-13}$ & 22.04 \\
\hline 39 & NP_249234.1 & & PA0543 & $4.70 \times 10^{-13}$ & 1438 \\
\hline
\end{tabular}




\begin{tabular}{|c|c|c|c|c|c|}
\hline Rank & Accession & $\begin{array}{l}\text { Gene } \\
\text { name }\end{array}$ & Protein name & $\mathrm{p}$ value & $\begin{array}{l}\text { Odds } \\
\text { ratio }\end{array}$ \\
\hline 40 & & & hemagglutinin (pseudogene) & $5.24 \times 10^{-13}$ & 26.58 \\
\hline 41 & NP_253664.1 & $\operatorname{aruI}$ & $\begin{array}{l}\text { 2-ketoarginine decarboxylase, } \\
\text { AruI }\end{array}$ & $5.86 \times 10^{-13}$ & 25.00 \\
\hline 42 & & & $\begin{array}{l}\text { aldehyde dehydrogenase } \\
\text { (pseudogene) }\end{array}$ & $1.18 \times 10^{-12}$ & NA \\
\hline 43 & NP_251618.1 & & PA2928 & $1.28 \times 10^{-12}$ & 13.76 \\
\hline 44 & NP_251285.1 & & PA2595 & $1.30 \times 10^{-12}$ & 8.36 \\
\hline 45 & NP_253633.1 & mutL & $\begin{array}{l}\text { DNA mismatch repair protein } \\
\text { MutL }\end{array}$ & $1.63 \times 10^{-12}$ & 15.24 \\
\hline 46 & & & $\begin{array}{l}\text { DeoR family transcriptional } \\
\text { regulator (pseudogene) }\end{array}$ & $2.45 \times 10^{-12}$ & NA \\
\hline 47 & NP_252760.1 & & PA4071 & $3.68 \times 10^{-12}$ & 11.86 \\
\hline 48 & & & $\begin{array}{l}\text { phosphoenolpyruvate } \\
\text { carboxylase (pseudogene) }\end{array}$ & $4.42 \times 10^{-12}$ & 57.97 \\
\hline 49 & & & $\begin{array}{l}\text { helix-turn-helix transcriptional } \\
\text { regulator (pseudogene) }\end{array}$ & $4.66 \times 10^{-12}$ & 58.68 \\
\hline 50 & & & $\begin{array}{l}\text { TetR family transcriptional } \\
\text { regulator (pseudogene) }\end{array}$ & $5.34 \times 10^{-12}$ & 11.32 \\
\hline 51 & NP_251292.1 & & $\begin{array}{l}\text { 3-mercaptopropionate } \\
\text { dioxygenase }\end{array}$ & $5.51 \times 10^{-12}$ & 9.07 \\
\hline 52 & NP_250800.1 & & PA2110 & $5.80 \times 10^{-12}$ & 7.69 \\
\hline 53 & NP_252377.1 & $p p c$ & $\begin{array}{l}\text { phosphoenolpyruvate } \\
\text { carboxylase }\end{array}$ & $6.17 \times 10^{-12}$ & NA \\
\hline 54 & NP_250181.1 & & $\begin{array}{l}\text { PA1490 probable transcriptional } \\
\text { regulator }\end{array}$ & $6.59 \times 10^{-12}$ & 79.04 \\
\hline 55 & & & nan & $6.84 \times 10^{-12}$ & 1217 \\
\hline 56 & & & $\begin{array}{l}\text { hypothetical protein } \\
\text { (pseudogene) }\end{array}$ & $8.86 \times 10^{-12}$ & 115.6 \\
\hline 57 & NP_248954.1 & $h c p C$ & secreted protein Hcp & $9.51 \times 10^{-12}$ & 0.21 \\
\hline 58 & NP_253650.1 & & PA4963 & $9.65 \times 10^{-12}$ & 106.3 \\
\hline 59 & & & nan & $9.85 \times 10^{-12}$ & 11.16 \\
\hline 60 & & & $\begin{array}{l}\text { high-affinity branched-chain } \\
\text { amino acid ABC transporter } \\
\text { ATP-binding protein LivG } \\
\text { (pseudogene) }\end{array}$ & $1.00 \times 10^{-11}$ & NA \\
\hline 61 & & & MFS transporter (pseudogene) & $1.19 \times 10^{-11}$ & 24.73 \\
\hline 62 & NP_252036.1 & $h s b R$ & $\begin{array}{l}\text { HptB-dependent secretion and } \\
\text { biofilm regulator HsbR }\end{array}$ & $1.20 \times 10^{-11}$ & 34.36 \\
\hline 63 & NP_252019.1 & & PA3329 & $1.95 \times 10^{-11}$ & 63.99 \\
\hline 64 & & & $\begin{array}{l}\text { hypothetical protein } \\
\text { (pseudogene) }\end{array}$ & $2.12 \times 10^{-11}$ & NA \\
\hline 65 & & & $\begin{array}{l}\text { hypothetical protein } \\
\text { (pseudogene) }\end{array}$ & $2.18 \times 10^{-11}$ & 11.21 \\
\hline 66 & & & [] & $2.38 \times 10^{-11}$ & 5.92 \\
\hline 67 & & & $\begin{array}{l}\text { pyridoxamine } 5^{\prime} \text {-phosphate } \\
\text { oxidase (pseudogene) }\end{array}$ & $3.70 \times 10^{-11}$ & 15.59 \\
\hline
\end{tabular}




\begin{tabular}{|c|c|c|c|c|c|}
\hline Rank & Accession & $\begin{array}{l}\text { Gene } \\
\text { name }\end{array}$ & Protein name & p value & $\begin{array}{l}\text { Odds } \\
\text { ratio }\end{array}$ \\
\hline 68 & & & $\begin{array}{l}\text { polyhydroxybutyrate } \\
\text { depolymerase (pseudogene) }\end{array}$ & $3.72 \times 10^{-11}$ & 1231 \\
\hline 69 & & & $\begin{array}{l}\text { cell division protein } \\
\text { (pseudogene) }\end{array}$ & $4.02 \times 10^{-11}$ & 17.61 \\
\hline 70 & NP_252240.1 & $\operatorname{alg} F$ & $\begin{array}{l}\text { alginate o-acetyltransferase } \\
\text { AlgF }\end{array}$ & $4.46 \times 10^{-11}$ & 21.32 \\
\hline 71 & & & nan & $6.46 \times 10^{-11}$ & 14.2 \\
\hline 72 & & & $\begin{array}{l}\text { alginate O-acetyltransferase } \\
\text { (pseudogene) }\end{array}$ & $7.28 \times 10^{-11}$ & 34.78 \\
\hline 73 & & & [] & $7.75 \times 10^{-11}$ & NA \\
\hline 74 & & & nan & $8.37 \times 10^{-11}$ & NA \\
\hline 75 & NP_249718.1 & $a m a B$ & $\begin{array}{l}\text { delta1-Piperideine-6- } \\
\text { carboxylate } \\
\text { dehydrogenase }\end{array}$ & $8.41 \times 10^{-11}$ & 15.9 \\
\hline 76 & & & nan & $8.70 \times 10^{-11}$ & 4.78 \\
\hline 77 & NP_249943.1 & $d p k A$ & DpkA & $9.23 \times 10^{-11}$ & 20.55 \\
\hline 78 & NP_251108.1 & & PA2418 & $1.13 \times 10^{-10}$ & 18.09 \\
\hline 79 & NP_249762.1 & braF & $\begin{array}{l}\text { branched-chain amino acid } \\
\text { transport protein BraF }\end{array}$ & $1.36 \times 10^{-10}$ & 59.39 \\
\hline 80 & & & $\begin{array}{l}\text { fused response } \\
\text { regulator/phosphatase } \\
\text { (pseudogene) }\end{array}$ & $1.37 \times 10^{-10}$ & 58.28 \\
\hline 81 & NP_250038.1 & & $\begin{array}{l}\text { PA1347 probable transcriptional } \\
\text { regulator }\end{array}$ & $1.47 \times 10^{-10}$ & 21.64 \\
\hline 82 & & & transporter (pseudogene) & $1.64 \times 10^{-10}$ & 1082 \\
\hline 83 & NP_253532.1 & $\operatorname{dipZ}$ & $\begin{array}{l}\text { thiol:disulfide interchange } \\
\text { protein DipZ }\end{array}$ & $1.66 \times 10^{-10}$ & 52.20 \\
\hline 84 & & & $\begin{array}{l}\text { amino acid } \mathrm{ABC} \text { transporter } \\
\text { permease (pseudogene) }\end{array}$ & $1.74 \times 10^{-10}$ & 53.29 \\
\hline 85 & NP_253820.1 & & PA5133 & $1.91 \times 10^{-10}$ & 10.41 \\
\hline 86 & & & hypothetical protein & $2.54 \times 10^{-10}$ & NA \\
\hline 87 & & & hypothetical protein & $2.54 \times 10^{-10}$ & NA \\
\hline 88 & & & hypothetical protein & $2.54 \times 10^{-10}$ & NA \\
\hline 89 & & & $\begin{array}{l}\text { DNA cytosine } \\
\text { methyltransferase (pseudogene) }\end{array}$ & $2.54 \times 10^{-10}$ & NA \\
\hline 90 & & & nan & $2.54 \times 10^{-10}$ & NA \\
\hline 91 & & & hypothetical protein & $2.54 \times 10^{-10}$ & NA \\
\hline 92 & & & $\begin{array}{l}\text { NAD-dependent deacylase } \\
\text { (pseudogene) }\end{array}$ & $2.54 \times 10^{-10}$ & NA \\
\hline 93 & & & integrase & $2.54 \times 10^{-10}$ & NA \\
\hline 94 & & & integrase & $2.54 \times 10^{-10}$ & NA \\
\hline 95 & & & hypothetical protein & $2.54 \times 10^{-10}$ & NA \\
\hline 96 & & & $\begin{array}{l}\text { general secretion pathway } \\
\text { protein GspH (pseudogene) }\end{array}$ & $2.54 \times 10^{-10}$ & NA \\
\hline 97 & & & hypothetical protein & $2.54 \times 10^{-10}$ & NA \\
\hline 98 & & & hypothetical protein & $2.54 \times 10^{-10}$ & NA \\
\hline 99 & & & hypothetical protein & $2.54 \times 10^{-10}$ & NA \\
\hline
\end{tabular}




\begin{tabular}{llllrr}
\hline Rank & Accession & $\begin{array}{c}\text { Gene } \\
\text { name }\end{array}$ & Protein name & p value & $\begin{array}{r}\text { Odds } \\
\text { ratio }\end{array}$ \\
\hline 100 & & hypothetical protein & $2.54 \times 10^{-10}$ & NA \\
101 & & $\begin{array}{l}\text { hypothetical protein } \\
\text { hypothetical protein } \\
102\end{array}$ & & $2.54 \times 10^{-10}$ & NA \\
103 & & $\begin{array}{l}\text { transcriptional regulator } \\
\text { (pseudogene) }\end{array}$ & $2.54 \times 10^{-10}$ & NA \\
& & PA0581 & $2.54 \times 10^{-10}$ & NA \\
104 & NP_249272.1 & PA0864 probable transcriptional & $2.54 \times 10^{-10}$ & 0 \\
105 & NP_249555.1 & & regulator & 0 \\
& & PA1044 & $2.54 \times 10^{-10}$ & 0 \\
106 & NP_249735.1 & Lip2 & $2.54 \times 10^{-10}$ & 0 \\
107 & NP_250357.1 & lip2 & PA2750 & $2.54 \times 10^{-10}$ & 0 \\
108 & NP_251440.1 & & PA3840 & $2.54 \times 10^{-10}$ & 0 \\
109 & NP_252529.1 & & & & 0 \\
\hline
\end{tabular}

\section{B.2. Dataset 1 treeWAS results}

Table 19: Genes identified with treeWAS from dataset 1, gene presence/absence.

\begin{tabular}{|c|c|c|c|c|c|}
\hline Rank & Accession & $\begin{array}{l}\text { Gene } \\
\text { name }\end{array}$ & Protein name & $\begin{array}{c}\text { Association } \\
\text { score }\end{array}$ & p value \\
\hline 1 & NP_250018.1 & & PA1327 probable protease & -50 & 0 \\
\hline 2 & NP_249374.1 & $h x c Y$ & $\mathrm{HxcY}$ & -49 & 0 \\
\hline 3 & NP_250230.1 & & PA1539 & -49 & 0 \\
\hline 4 & NP_250420.1 & & PA1729 & -48 & 0 \\
\hline 5 & NP_249865.1 & nap A & $\begin{array}{l}\text { periplasmic nitrate reductase } \\
\text { protein NapA }\end{array}$ & -46 & 0 \\
\hline 6 & NP_250376.1 & mas $A$ & enolase-phosphatase E-1 & -46 & 0 \\
\hline 7 & NP_248888.1 & tonB2 & tonB2 & -45 & 0 \\
\hline 8 & NP_251618.1 & & PA2928 & -45 & 0 \\
\hline 9 & NP_064723.1 & recF & recF & -43 & 0 \\
\hline 10 & & & nan & 42 & 0 \\
\hline 11 & NP_249603.1 & & PA0912 & -40 & 0 \\
\hline 12 & & & hypothetical protein & 37 & 0 \\
\hline 13 & NP_250764.1 & & PA2074 & 37 & 0 \\
\hline 14 & NP_251800.1 & & PA3110 & -37 & 0 \\
\hline 15 & NP_253724.1 & & PA5037 & -35 & 0 \\
\hline 16 & NP_251981.1 & tli1 & Tli1 & 34 & 0 \\
\hline 17 & & & [] & 33 & 0 \\
\hline 18 & & & putative rubredoxin reductase & 33 & 0 \\
\hline 19 & & & hypothetical protein & 30 & 0 \\
\hline 20 & & & nan & 30 & 0 \\
\hline 21 & & & $\begin{array}{l}\text { ABC transporter } \\
\text { substrate-binding protein }\end{array}$ & 29 & 0 \\
\hline 22 & & & $\begin{array}{l}\text { mammalian cell entry protein } \\
\text { (pseudogene) }\end{array}$ & 28 & 0 \\
\hline 23 & & & $\begin{array}{l}\text { hypothetical protein } \\
\text { (pseudogene) }\end{array}$ & 28 & 0 \\
\hline 24 & NP_248731.1 & & PA0041 probable hemagglutinin & -28 & 0 \\
\hline
\end{tabular}




\begin{tabular}{|c|c|c|c|c|c|}
\hline Rank & Accession & $\begin{array}{l}\text { Gene } \\
\text { name }\end{array}$ & Protein name & $\begin{array}{c}\text { Association } \\
\text { score }\end{array}$ & p value \\
\hline 25 & & & hypothetical protein & 27 & 0 \\
\hline 26 & & & hypothetical protein & 27 & 0 \\
\hline 27 & & & glyA & 26 & 0 \\
\hline 28 & & & hypothetical protein & 26 & 0 \\
\hline 29 & NP_250105.1 & & 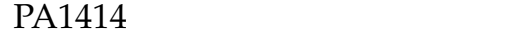 & 26 & 0 \\
\hline 30 & NP_250632.1 & & PA1942 & 26 & 0 \\
\hline 31 & & & hypothetical protein & 25 & 0 \\
\hline 32 & & & cytidine deaminase & 25 & 0 \\
\hline 33 & & & hypothetical protein & 25 & 0 \\
\hline 34 & & & hypothetical protein & 25 & 0 \\
\hline 35 & & & DNA repair protein & 25 & 0 \\
\hline 36 & & & glycosyl hydrolase & 25 & 0 \\
\hline 37 & & & $\begin{array}{l}\text { hypothetical protein } \\
\text { (pseudogene) }\end{array}$ & 24 & 0 \\
\hline 38 & & & $\begin{array}{l}\text { hypothetical protein } \\
\text { (pseudogene) }\end{array}$ & 24 & 0 \\
\hline 39 & NP_248954.1 & $h с p C$ & secreted protein Hcp & -24 & 0 \\
\hline 40 & NP_251089.1 & $p v d D$ & pyoverdine synthetase $\mathrm{D}$ & -24 & 0 \\
\hline 41 & NP_251148.1 & & PA2458 & -24 & 0 \\
\hline 42 & NP_251175.1 & & PA2485 & 24 & 0 \\
\hline 43 & YP_003933614.1fi & $f d n G$ & $\begin{array}{l}\text { formate dehydrogenase-O, } \\
\text { major subunit }\end{array}$ & -24 & 0 \\
\hline 44 & & & hypothetical protein & 23 & 0 \\
\hline 45 & & & $\begin{array}{l}\text { TetR family transcriptional } \\
\text { regulator (pseudogene) }\end{array}$ & 23 & 0 \\
\hline 46 & & & hypothetical protein & 23 & 0 \\
\hline 47 & & & hypothetical protein & 23 & 0 \\
\hline 48 & & & hypothetical protein & 23 & 0 \\
\hline 49 & & & hypothetical protein & 23 & 0 \\
\hline 50 & & & hypothetical protein & 23 & 0 \\
\hline 51 & & & hypothetical protein & 23 & 0 \\
\hline 52 & & & hypothetical protein & 23 & 0 \\
\hline 53 & & & hypothetical protein & 23 & 0 \\
\hline 54 & & & ffs & 23 & 0 \\
\hline 55 & & & hypothetical protein & 23 & 0 \\
\hline 56 & & & cytochrome $\mathrm{C}$ oxidase subunit II & 23 & 0 \\
\hline 57 & & & universal stress protein & 23 & 0 \\
\hline 58 & & & hypothetical protein & 23 & 0 \\
\hline 59 & & & $\begin{array}{l}\text { nitrate reductase catalytic } \\
\text { subunit (pseudogene) }\end{array}$ & 22 & 0 \\
\hline 60 & & & [] & 22 & 0 \\
\hline 61 & & & $\begin{array}{l}\text { transcriptional regulator } \\
\text { (pseudogene) }\end{array}$ & 22 & 0 \\
\hline 62 & & & hypothetical protein & -22 & 0 \\
\hline 63 & & & hypothetical protein & 22 & 0 \\
\hline 64 & & & hypothetical protein & 22 & 0 \\
\hline 65 & & & hypothetical protein & -22 & 0 \\
\hline 66 & & & hypothetical protein & 22 & 0 \\
\hline
\end{tabular}




\begin{tabular}{|c|c|c|c|c|c|}
\hline Rank & Accession & $\begin{array}{l}\text { Gene } \\
\text { name }\end{array}$ & Protein name & $\begin{array}{c}\text { Association } \\
\text { score }\end{array}$ & p value \\
\hline 67 & & & phosphoenolpyruvate synthase & 22 & 0 \\
\hline 68 & & & hypothetical protein & 22 & 0 \\
\hline 69 & & & hypothetical protein & 22 & 0 \\
\hline 70 & & & hypothetical protein & 22 & 0 \\
\hline 71 & & & hypothetical protein & 22 & 0 \\
\hline 72 & & & hypothetical protein & 22 & 0 \\
\hline 73 & & & ferredoxin (pseudogene) & 22 & 0 \\
\hline 74 & & & monoamine oxidase & 22 & 0 \\
\hline 75 & & & hypothetical protein & 22 & 0 \\
\hline 76 & NP_250710.1 & mexZ & MexZ & -22 & 0 \\
\hline 77 & & & aminotransferase & 21 & 0 \\
\hline 78 & & & recombinase XerC & 21 & 0 \\
\hline 79 & & & nan & 21 & 0 \\
\hline 80 & & & aminopeptidase (pseudogene) & 21 & 0 \\
\hline 81 & & & hypothetical protein & 21 & 0 \\
\hline 82 & & & hypothetical protein & 21 & 0 \\
\hline 83 & & & hypothetical protein & 21 & 0 \\
\hline 84 & & & hypothetical protein & 21 & 0 \\
\hline 85 & & & hypothetical protein & 21 & 0 \\
\hline 86 & & & hypothetical protein & 21 & 0 \\
\hline 87 & & & hypothetical protein & 21 & 0 \\
\hline 88 & & & hypothetical protein & 21 & 0 \\
\hline 89 & & & $\begin{array}{l}\text { hypothetical protein } \\
\text { (pseudogene) }\end{array}$ & 21 & 0 \\
\hline 90 & & & hypothetical protein & -21 & 0 \\
\hline 91 & & & hypothetical protein & 21 & 0 \\
\hline 92 & & & hypothetical protein & 21 & 0 \\
\hline 93 & & & transporter & 21 & 0 \\
\hline 94 & & & hypothetical protein & 21 & 0 \\
\hline 95 & & & $\begin{array}{l}\text { hypothetical protein } \\
\text { (pseudogene) }\end{array}$ & 21 & 0 \\
\hline 96 & & & ATP-binding protein & 21 & 0 \\
\hline 97 & NP_250674.1 & exaC & $\begin{array}{l}\text { NAD+ dependent aldehyde } \\
\text { dehydrogenase ExaC }\end{array}$ & -21 & 0 \\
\hline 98 & NP_253378.1 & & PA4689 & -21 & 0 \\
\hline 99 & & & 3-oxoacyl-ACP synthase & 20 & 0 \\
\hline 100 & & & hypothetical protein & 20 & 0 \\
\hline 101 & & & $\begin{array}{l}\text { non-ribosomal peptide } \\
\text { synthetase (pseudogene) }\end{array}$ & 20 & 0 \\
\hline 102 & & & hypothetical protein & -20 & 0 \\
\hline 103 & & & flagellar export chaperone FliS & 20 & 0 \\
\hline 104 & & & protease (pseudogene) & 20 & 0 \\
\hline 105 & & & hypothetical protein & 20 & 0 \\
\hline 106 & & & hypothetical protein & 20 & 0 \\
\hline 107 & & & $\begin{array}{l}\text { response regulator GacA } \\
\text { (pseudogene) }\end{array}$ & 20 & 0 \\
\hline 108 & & & hypothetical protein & 20 & 0 \\
\hline 109 & & & hypothetical protein & 20 & 0 \\
\hline
\end{tabular}




\begin{tabular}{|c|c|c|c|c|c|}
\hline Rank & Accession & $\begin{array}{l}\text { Gene } \\
\text { name }\end{array}$ & Protein name & $\begin{array}{c}\text { Association } \\
\text { score }\end{array}$ & p value \\
\hline 110 & & & hypothetical protein & 20 & 0 \\
\hline 111 & & & acyl carrier protein & 20 & 0 \\
\hline 112 & & & methionine aminopeptidase & -20 & 0 \\
\hline 113 & & & methionine aminopeptidase & 20 & 0 \\
\hline 114 & & & hypothetical protein & 20 & 0 \\
\hline 115 & & & ffs & 20 & 0 \\
\hline 116 & & & $\begin{array}{l}\text { hypothetical protein } \\
\text { (pseudogene) }\end{array}$ & 20 & 0 \\
\hline 117 & & & hypothetical protein & 20 & 0 \\
\hline 118 & & & $\begin{array}{l}\text { hypothetical protein } \\
\text { (pseudogene) }\end{array}$ & 20 & 0 \\
\hline 119 & & & hypothetical protein & 20 & 0 \\
\hline 120 & & & hypothetical protein & 20 & 0 \\
\hline 121 & & & hypothetical protein & 20 & 0 \\
\hline 122 & & & beta-lactamase & -20 & 0 \\
\hline 123 & & & hypothetical protein & 20 & 0 \\
\hline 124 & & & nan & 20 & 0 \\
\hline 125 & & & nan & 20 & 0 \\
\hline 126 & & & [] & 20 & 0 \\
\hline 127 & NP_249785.1 & fliD & flagellar capping protein FliD & -20 & 0 \\
\hline 128 & NP_250876 & & PA2186 & -20 & 0 \\
\hline 129 & NP_251152.1 & & PA2462 & -20 & 0 \\
\hline
\end{tabular}

\section{B.3. Dataset 1 random forest results}

Table 20: Genes identified with RF from dataset 1, gene presence/absence. Genes with importances greater than or equal to 0.002 are reported.

\begin{tabular}{|c|c|c|c|c|}
\hline Rank & Accession & Gene name & Protein name & Importance \\
\hline 1 & NP_250764.1 & & PA2074 & 0.237 \\
\hline 2 & NP_249454.1 & muсA & anti-sigma factor MucA & 0.123 \\
\hline 3 & NP_248888.1 & tonB2 & TonB2 & 0.084 \\
\hline 4 & NP_253724.1 & & PA5037 & 0.041 \\
\hline 5 & NP_064723.1 & $\operatorname{rec} F$ & RecF protein & 0.029 \\
\hline 6 & NP_252532 & & PA3843 & 0.022 \\
\hline 7 & & & $\begin{array}{l}\text { non-ribosomal peptide synthetase } \\
\text { (pseudogene) }\end{array}$ & 0.015 \\
\hline 8 & NP_251184.1 & $\operatorname{mexF}$ & $\begin{array}{l}\text { Resistance-Nodulation-Cell } \\
\text { Division (RND) multidrug efflux } \\
\text { transporter MexF }\end{array}$ & 0.011 \\
\hline 9 & & & $\begin{array}{l}\text { DNA-binding response regulator } \\
\text { (pseudogene) }\end{array}$ & 0.01 \\
\hline 10 & NP_249929.1 & & $\begin{array}{l}\text { PA1238 probable outer membrane } \\
\text { component of multidrug efflux } \\
\text { pump }\end{array}$ & 0.009 \\
\hline 11 & & & mutL (pseudogene) & 0.009 \\
\hline 12 & NP_249381.1 & $p d t A$ & $\begin{array}{l}\text { phosphate depletion regulated TPS } \\
\text { partner A, PdtA }\end{array}$ & 0.008 \\
\hline
\end{tabular}




\begin{tabular}{|c|c|c|c|c|}
\hline Rank & Accession & Gene name & Protein name & Importance \\
\hline 13 & NP 253820.1 & & PA5133 & 0.008 \\
\hline 14 & NP_248948.1 & & PA0257 & 0.006 \\
\hline 15 & NP_250118 & & PA1427 & 0.006 \\
\hline 16 & NP_251134.1 & glyA2 & serine hydroxymethyltransferase & 0.005 \\
\hline 17 & NP_249415.1 & & $\begin{array}{l}\text { PA0724 probable coat protein A of } \\
\text { bacteriophage Pf1 }\end{array}$ & 0.005 \\
\hline 18 & NP_250074.1 & & PA1383 & 0.005 \\
\hline 19 & & & hypothetical protein & 0.005 \\
\hline 20 & NP_252177.1 & tle5 & Tle5 & 0.005 \\
\hline 21 & & & $\begin{array}{l}\text { TetR family transcriptional } \\
\text { regulator (pseudogene) }\end{array}$ & 0.005 \\
\hline 22 & NP_249374.1 & $h x_{c} Y$ & $\mathrm{HxcY}$ & 0.004 \\
\hline 23 & NP_249842.1 & imm2 & pyocin S2 immunity protein & 0.004 \\
\hline 24 & & & nan & 0.004 \\
\hline 25 & NP_250018.1 & & PA1327 probable protease & 0.004 \\
\hline 26 & NP_253474.1 & & $\begin{array}{l}\text { PA4786 probable short-chain } \\
\text { dehydrogenase }\end{array}$ & 0.004 \\
\hline 27 & & & nan & 0.004 \\
\hline 28 & NP_250030.1 & aatP & AatP & 0.004 \\
\hline 29 & NP_249843 & & PA1152 & 0.004 \\
\hline 30 & NP_248851 & & PA0161 & 0.004 \\
\hline 31 & NP_250980.1 & $g c d$ & glucose dehydrogenase & 0.004 \\
\hline 32 & NP_253775.1 & & PA5088 & 0.003 \\
\hline 33 & & & hypothetical protein & 0.003 \\
\hline 34 & NP_253847.1 & & drug efflux transporter & 0.003 \\
\hline 35 & & & transcriptional regulator & 0.003 \\
\hline 36 & NP_249865.1 & napA & $\begin{array}{l}\text { periplasmic nitrate reductase } \\
\text { protein NapA }\end{array}$ & 0.003 \\
\hline 37 & & & peptidase & 0.003 \\
\hline 38 & NP_249683.1 & сирC1 & fimbrial subunit CupC1 & 0.003 \\
\hline 39 & & & $\begin{array}{l}\text { cystine } \mathrm{ABC} \text { transporter } \\
\text { substrate-binding protein } \\
\text { (pseudogene) }\end{array}$ & 0.003 \\
\hline 40 & NP_250246.1 & ccoP2 & $\begin{array}{l}\text { Cytochrome c oxidase, cbb3-type, } \\
\text { CcoP subunit }\end{array}$ & 0.003 \\
\hline 41 & NP_253337.1 & cupE1 & Pilin subunit CupE1 & 0.003 \\
\hline 42 & NP_253159.1 & & PA4469 & 0.003 \\
\hline 43 & NP_249234.1 & & PA0543 & 0.003 \\
\hline 44 & NP_249116.1 & $\operatorname{mex} A$ & $\begin{array}{l}\text { Resistance-Nodulation-Cell } \\
\text { Division (RND) multidrug efflux } \\
\text { membrane fusion protein MexA } \\
\text { precursor }\end{array}$ & 0.003 \\
\hline 45 & & & hypothetical protein & 0.003 \\
\hline 46 & NP_252799.1 & $\operatorname{ampC}$ & beta-lactamase precursor & 0.003 \\
\hline 47 & NP_248963.1 & & $\begin{array}{l}\text { PA0272 probable transcriptional } \\
\text { regulator }\end{array}$ & 0.003 \\
\hline 48 & & & hypothetical protein (pseudogene) & 0.003 \\
\hline 49 & & & DNA-binding protein & 0.003 \\
\hline 50 & & & 16S ribosomal RNA & 0.003 \\
\hline
\end{tabular}




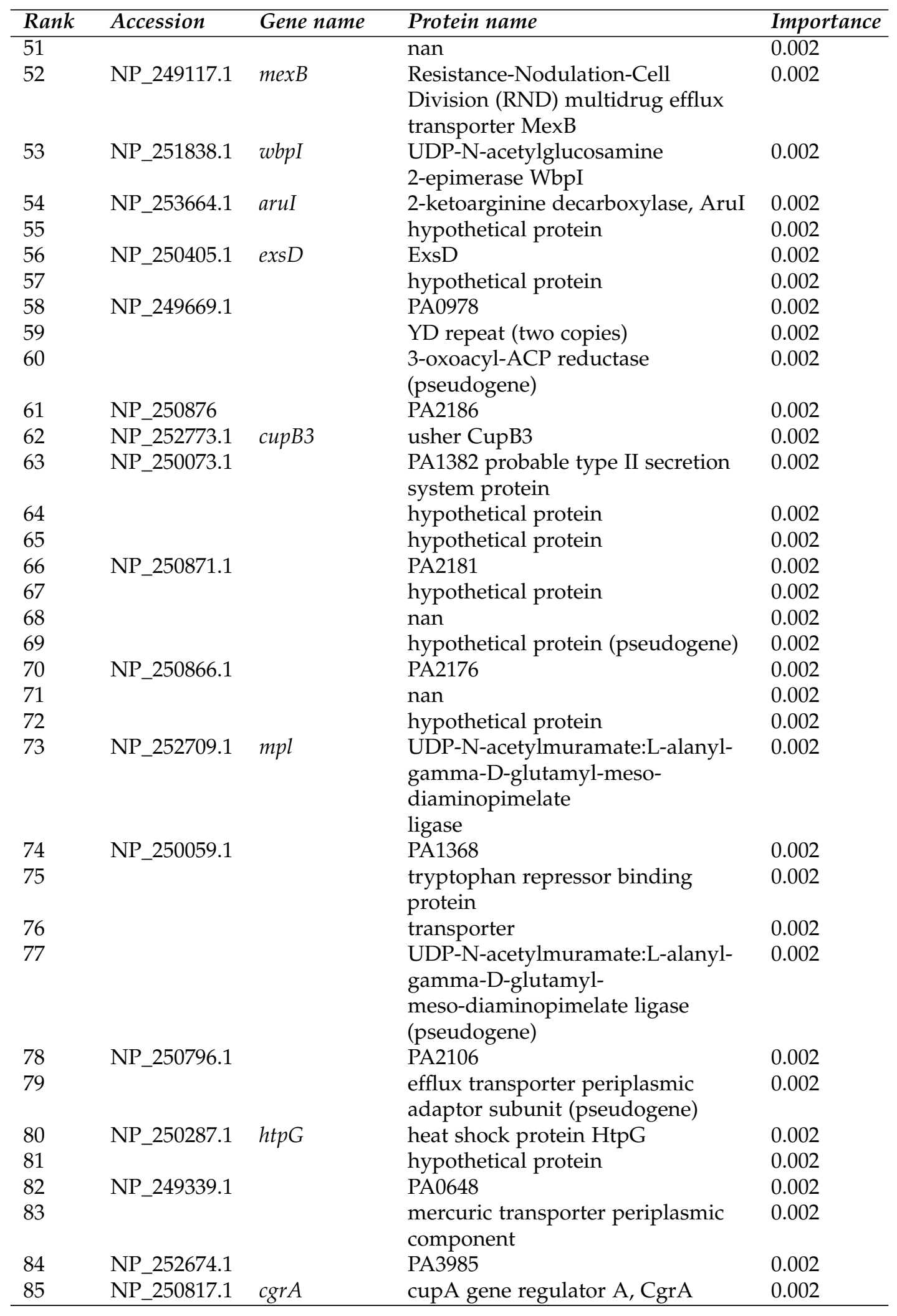




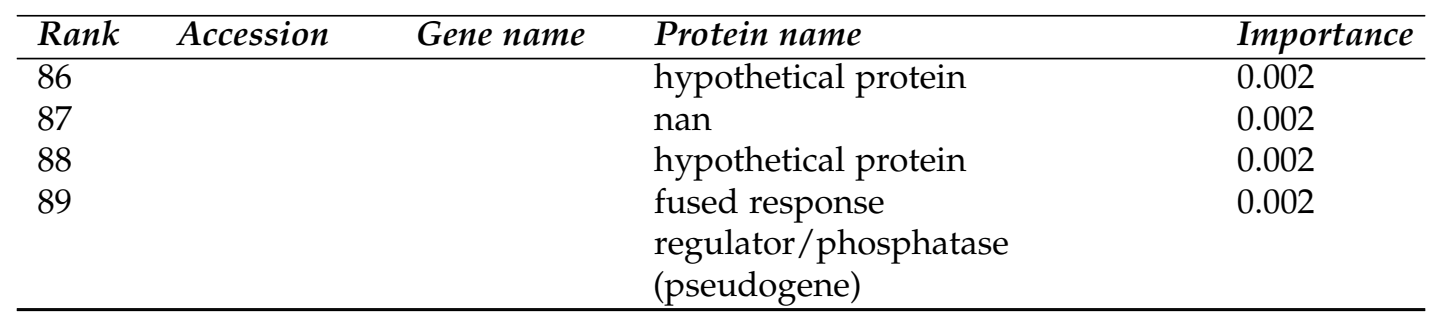

\section{B.4. Dataset 1 support vector machine results}

Table 21: Genes identified with SVM from dataset 1, gene presence/absence. Top 100 genes with absolute hyperplane coefficients greater than 0.03 are reported.

\begin{tabular}{|c|c|c|c|c|}
\hline Rank & Accession & Gene name & Protein name & Importance \\
\hline 1 & NP_249454.1 & muсA & anti-sigma factor MucA & -0.0896 \\
\hline 2 & NP_251876.1 & oprB & $\begin{array}{l}\text { Glucose/carbohydrate outer } \\
\text { membrane porin OprB precursor }\end{array}$ & -0.0875 \\
\hline 3 & NP_250710.1 & $\operatorname{mexZ}$ & MexZ & -0.0727 \\
\hline 4 & NP_252903.1 & phzE1 & $\begin{array}{l}\text { phenazine biosynthesis protein } \\
\text { PhzE }\end{array}$ & -0.0654 \\
\hline 5 & NP_251417 & & PA2727 & -0.0619 \\
\hline 6 & NP_248888.1 & tonB2 & TonB2 & -0.0584 \\
\hline 7 & & & nan & 0.0574 \\
\hline 8 & & & nan & -0.0535 \\
\hline 9 & NP_251836.1 & $w b p K$ & $\begin{array}{l}\text { probable NAD-dependent } \\
\text { epimerase/dehydratase } \mathrm{WbpK}\end{array}$ & -0.0523 \\
\hline 10 & NP_253378.1 & & PA4689 & -0.0495 \\
\hline 11 & & & hypothetical protein & 0.0488 \\
\hline 12 & & & hypothetical protein & 0.0487 \\
\hline 13 & NP_251134.1 & glyA2 & serine hydroxymethyltransferase & -0.0484 \\
\hline 14 & & & tRNA-Ile & -0.0484 \\
\hline 15 & & & $\begin{array}{l}\text { RNA polymerase subunit sigma } \\
\text { (pseudogene) }\end{array}$ & 0.0484 \\
\hline 16 & & & $\begin{array}{l}\text { aldehyde dehydrogenase } \\
\text { (pseudogene) }\end{array}$ & 0.0470 \\
\hline 17 & & & elongation factor $\mathrm{Tu}$ & 0.0468 \\
\hline 18 & & & transcriptional regulator & 0.0453 \\
\hline 19 & NP_252906.1 & phzG1 & $\begin{array}{l}\text { probable pyridoxamine } \\
5^{\prime} \text {-phosphate oxidase }\end{array}$ & -0.0448 \\
\hline 20 & & & tRNA-Ala & -0.0447 \\
\hline 21 & NP_249374.1 & $h x_{c} Y$ & $\mathrm{HxcY}$ & -0.0443 \\
\hline 22 & NP_249336.1 & & PA0645 & 0.0441 \\
\hline 23 & NP_251148.1 & & PA2458 & -0.0441 \\
\hline 24 & NP_251147.1 & & PA2457 & -0.0440 \\
\hline 25 & & & hypothetical protein (pseudogene) & -0.0431 \\
\hline 26 & & & $\begin{array}{l}\text { non-ribosomal peptide synthetase } \\
\text { (pseudogene) }\end{array}$ & -0.0430 \\
\hline 27 & & & nan & -0.0428 \\
\hline 28 & NP_250922.1 & pslB & PslB & -0.0427 \\
\hline
\end{tabular}




\begin{tabular}{|c|c|c|c|c|}
\hline Rank & Accession & Gene name & Protein name & Importance \\
\hline 29 & NP_249416.1 & & $\begin{array}{l}\text { PA0725 hypothetical protein of } \\
\text { bacteriophage Pf1 }\end{array}$ & -0.0426 \\
\hline 30 & NP_251800.1 & & PA3110 & -0.0426 \\
\hline 31 & & & $\begin{array}{l}\text { TetR family transcriptional } \\
\text { regulator (pseudogene) }\end{array}$ & 0.0424 \\
\hline 32 & NP_249413.1 & & $\begin{array}{l}\text { PA0722 hypothetical protein of } \\
\text { bacteriophage Pf1 }\end{array}$ & -0.0415 \\
\hline 33 & NP_249409.1 & & $\begin{array}{l}\text { PA0718 hypothetical protein of } \\
\text { bacteriophage Pf1 }\end{array}$ & -0.0413 \\
\hline 34 & NP_251756.1 & & PA3066 & -0.04052 \\
\hline 35 & NP_251757.1 & & $\begin{array}{l}\text { PA3067 probable transcriptional } \\
\text { regulator }\end{array}$ & -0.0405 \\
\hline 36 & & & nan & 0.0404 \\
\hline 37 & & & hypothetical protein & 0.0398 \\
\hline 38 & & & mutL (pseudogene) & 0.0396 \\
\hline 39 & & & hypothetical protein & -0.0395 \\
\hline 40 & & & nan & -0.0394 \\
\hline 41 & NP_250914.1 & & PA2224 & -0.0393 \\
\hline 42 & NP_249414.1 & $c o a B$ & coat protein B of bacteriophage Pf1 & -0.0391 \\
\hline 43 & NP_252888.1 & & $\begin{array}{l}\text { PA4199 probable acyl-CoA } \\
\text { dehydrogenase }\end{array}$ & -0.0390 \\
\hline 44 & & & nan & 0.0385 \\
\hline 45 & & & $\begin{array}{l}\text { mammalian cell entry protein } \\
\text { (pseudogene) }\end{array}$ & 0.0385 \\
\hline 46 & & & nan & 0.0383 \\
\hline 47 & NP_253773 & & PA5086 & 0.0381 \\
\hline 48 & YP_003933611 & $1 c c o Q 2$ & $\begin{array}{l}\text { Cytochrome c oxidase, cbb3-type, } \\
\text { CcoQ subunit }\end{array}$ & 0.0377 \\
\hline 49 & & & tRNA-Gly & -0.0377 \\
\hline 50 & & & hypothetical protein & 0.0376 \\
\hline 51 & NP_253724.1 & & PA5037 & -0.0376 \\
\hline 52 & & & hypothetical protein & -0.0373 \\
\hline 53 & NP_251122.1 & $\operatorname{bexR}$ & bistable expression regulator, BexR & -0.0372 \\
\hline 54 & NP_249841.1 & pys2 & pyocin S2 & -0.0371 \\
\hline 55 & & & nan & 0.0367 \\
\hline 56 & NP_251418 & & PA2728 & 0.0366 \\
\hline 57 & NP_251755.1 & & PA3065 & -0.0364 \\
\hline 58 & & & hypothetical protein & -0.0364 \\
\hline 59 & & & hypothetical protein & -0.0362 \\
\hline 60 & NP_249408 & & $\begin{array}{l}\text { PA0717 hypothetical protein of } \\
\text { bacteriophage Pf1 }\end{array}$ & -0.0359 \\
\hline 61 & & & hypothetical protein & 0.0355 \\
\hline 62 & & & hypothetical protein & 0.0354 \\
\hline 63 & NP_249252.1 & & PA0561 & 0.0354 \\
\hline 64 & & & N-acetyltransferase GCN5 & 0.0352 \\
\hline 65 & & & nan & -0.0351 \\
\hline 66 & & & hypothetical protein & 0.0350 \\
\hline 67 & & & 16S ribosomal RNA & -0.0347 \\
\hline 68 & NP_252902.1 & phzD1 & $\begin{array}{l}\text { phenazine biosynthesis protein } \\
\text { PhzD }\end{array}$ & -0.0346 \\
\hline
\end{tabular}




\begin{tabular}{|c|c|c|c|c|}
\hline Rank & Accession & Gene name & Protein name & Importance \\
\hline 69 & & & hypothetical protein & 0.0346 \\
\hline 70 & NP_252711.1 & $h d h A$ & hydrazone dehydrogenase, HdhA & -0.0345 \\
\hline 71 & NP_253775.1 & & PA5088 & -0.0345 \\
\hline 72 & NP_252900.1 & phzB1 & $\begin{array}{l}\text { probable phenazine biosynthesis } \\
\text { protein }\end{array}$ & 0.0344 \\
\hline 73 & NP_253000.1 & $p c t B$ & chemotactic transducer PctB & 0.0341 \\
\hline 74 & & & $\begin{array}{l}\text { group II intron reverse } \\
\text { transcriptase/maturase }\end{array}$ & 0.0338 \\
\hline 75 & NP_250632.1 & & PA1942 & 0.0337 \\
\hline 76 & & & tRNA-Arg & -0.0337 \\
\hline 77 & NP_251835.1 & $w b p L$ & glycosyltransferase WbpL & 0.0335 \\
\hline 78 & & & [] & 0.0333 \\
\hline 79 & & & hypothetical protein & -0.0332 \\
\hline 80 & & & hypothetical protein & -0.0332 \\
\hline 81 & NP_249381.1 & $p d t A$ & $\begin{array}{l}\text { phosphate depletion regulated TPS } \\
\text { partner A, PdtA }\end{array}$ & -0.0331 \\
\hline 82 & & & $\begin{array}{l}\text { Rhs element Vgr protein } \\
\text { (pseudogene) }\end{array}$ & -0.0331 \\
\hline 83 & NP_249116.1 & $\operatorname{mex} A$ & $\begin{array}{l}\text { Resistance-Nodulation-Cell } \\
\text { Division (RND) multidrug efflux } \\
\text { membrane fusion protein MexA } \\
\text { precursor }\end{array}$ & -0.0330 \\
\hline 84 & & & hypothetical protein & 0.0327 \\
\hline 85 & & & nan & 0.0325 \\
\hline 86 & & & hypothetical protein & -0.0325 \\
\hline 87 & NP_253954.1 & $h с p B$ & secreted protein Hcp & -0.0324 \\
\hline 88 & & & $5 \mathrm{~S}$ ribosomal RNA & 0.0324 \\
\hline 89 & & & integrase & 0.0324 \\
\hline 90 & NP_251020.1 & & PA2330 & -0.0323 \\
\hline 91 & & & $\begin{array}{l}\text { UDP-N-acetylmuramate:L-alanyl- } \\
\text { gamma-D-glutamyl- } \\
\text { meso-diaminopimelate ligase } \\
\text { (pseudogene) }\end{array}$ & 0.0322 \\
\hline 92 & NP_249117.1 & $\operatorname{mex} B$ & $\begin{array}{l}\text { Resistance-Nodulation-Cell } \\
\text { Division (RND) multidrug efflux } \\
\text { transporter MexB }\end{array}$ & -0.0322 \\
\hline 93 & & & hypothetical protein & 0.0322 \\
\hline 94 & & & nan & 0.0322 \\
\hline 95 & & & nucleotide pyrophosphohydrolase & -0.0321 \\
\hline 96 & & & $\begin{array}{l}\text { phenazine biosynthesis protein } \\
\text { phzD }\end{array}$ & 0.0321 \\
\hline 97 & & & hypothetical protein (pseudogene) & -0.0320 \\
\hline 98 & & & hypothetical protein & -0.0320 \\
\hline 99 & & & nan & -0.0318 \\
\hline 100 & & & paraquat-inducible protein $\mathrm{A}$ & 0.0318 \\
\hline
\end{tabular}

\section{B.5. Dataset 2 PLINK results}


Table 22: SNPs identified with PLINK from dataset 2, SNPs within core genes. Top 100 SNPs with $p$ values less than $1 \times 10^{-8}$ are reported.

\begin{tabular}{|c|c|c|c|c|c|c|}
\hline Rank & Accession & $\begin{array}{l}\text { Gene } \\
\text { name }\end{array}$ & Protein name & Mutation & p value & $\begin{array}{l}\text { Odds } \\
\text { ratio }\end{array}$ \\
\hline 1 & NP_253781.1 & & $\begin{array}{l}\text { PA5094 probable } \\
\text { ATP-binding component } \\
\text { of ABC transporter }\end{array}$ & $\begin{array}{l}269 \\
\text { deletion }\end{array}$ & $3.321 \times 10^{-27}$ & 0.78 \\
\hline 2 & NP_253781.1 & & $\begin{array}{l}\text { PA5094 probable } \\
\text { ATP-binding component } \\
\text { of ABC transporter }\end{array}$ & X277Q & $3.321 \times 10^{-27}$ & 0.78 \\
\hline 3 & NP_253957.1 & & PA5270 & S5A & $2.598 \times 10^{-25}$ & 9.21 \\
\hline 4 & NP_253462.1 & & PA4774 & $\begin{array}{l}308 \\
\text { deletion }\end{array}$ & $1.26 \times 10^{-22}$ & 0.10 \\
\hline 5 & NP_253462.1 & & PA4774 & X350E & $1.26 \times 10^{-22}$ & 0.10 \\
\hline 6 & NP_252321.1 & & PA3631 & $\begin{array}{l}377 \\
\text { deletion }\end{array}$ & $1.866 \times 10^{-22}$ & 0.10 \\
\hline 7 & NP_252321.1 & & PA3631 & X409L & $1.866 \times 10^{-22}$ & 0.10 \\
\hline 8 & NP_253373.1 & & PA4684 & $\begin{array}{l}407 \\
\text { deletion }\end{array}$ & $3.157 \times 10^{-21}$ & 0.11 \\
\hline 9 & NP_253373.1 & & PA4684 & X433L & $3.157 \times 10^{-21}$ & 0.11 \\
\hline 10 & NP_250663.1 & $p q q F$ & $\begin{array}{l}\text { pyrroloquinoline quinone } \\
\text { biosynthesis protein } \mathrm{F}\end{array}$ & $\mathrm{H} 12 \mathrm{R}$ & $4.306 \times 10^{-21}$ & 0.14 \\
\hline 11 & NP_253159.1 & & PA4469 & P137S & $2.914 \times 10^{-19}$ & NA \\
\hline 12 & NP_253284.1 & & $\begin{array}{l}\text { PA4594 probable } \\
\text { ATP-binding component } \\
\text { of ABC transporter }\end{array}$ & A127G & $1.146 \times 10^{-18}$ & 2017 \\
\hline 13 & NP_253284.1 & & $\begin{array}{l}\text { PA4594 probable } \\
\text { ATP-binding component } \\
\text { of ABC transporter }\end{array}$ & T213A & $2.145 \times 10^{-18}$ & 2009 \\
\hline 14 & NP_253284.1 & & $\begin{array}{l}\text { PA4594 probable } \\
\text { ATP-binding component } \\
\text { of ABC transporter }\end{array}$ & A226T & $2.514 \times 10^{-18}$ & 2004 \\
\hline 15 & NP_253284.1 & & $\begin{array}{l}\text { PA4594 probable } \\
\text { ATP-binding component } \\
\text { of ABC transporter }\end{array}$ & L101M & $2.832 \times 10^{-18}$ & 2003 \\
\hline 16 & NP_250036.1 & & PA1345 & L17P & $4.235 \times 10^{-18}$ & 0.12 \\
\hline 17 & NP_253284.1 & & $\begin{array}{l}\text { PA4594 probable } \\
\text { ATP-binding component } \\
\text { of ABC transporter }\end{array}$ & $\mathrm{H} 220 \mathrm{D}$ & $5.11 \times 10^{-18}$ & 0.1991 \\
\hline 18 & NP_250663.1 & $p q q F$ & $\begin{array}{l}\text { pyrroloquinoline quinone } \\
\text { biosynthesis protein } \mathrm{F}\end{array}$ & H3R & $6.543 \times 10^{-18}$ & 0.17 \\
\hline 19 & NP_251048.1 & & PA2358 & $\mathrm{L} 2 \mathrm{P}$ & $2.17 \times 10^{-18}$ & 19.34 \\
\hline 20 & NP_252696.1 & proA & $\begin{array}{l}\text { gamma-glutamyl } \\
\text { phosphate reductase }\end{array}$ & R275Q & $6.07 \times 10^{-14}$ & 0.07 \\
\hline 21 & NP_250046.1 & & PA1355 & C37G & $1.67 \times 10^{-13}$ & 18.06 \\
\hline 22 & NP_249234.1 & & PA0543 & S64P & $2.32 \times 10^{-13}$ & 85.03 \\
\hline 23 & NP_249705.1 & wapB & $\begin{array}{l}\text { 1,2-glucosyltransferase } \\
\text { WapB }\end{array}$ & $\mathrm{D} 12 \mathrm{~S}$ & $3.97 \times 10^{-13}$ & 91.89 \\
\hline
\end{tabular}


B. RESULTS

\begin{tabular}{|c|c|c|c|c|c|c|}
\hline Rank & Accession & $\begin{array}{l}\text { Gene } \\
\text { name }\end{array}$ & Protein name & Mutation & p value & $\begin{array}{l}\text { Odds } \\
\text { ratio }\end{array}$ \\
\hline 24 & NP_249705.1 & wapB & $\begin{array}{l}\text { 1,2-glucosyltransferase } \\
\text { WapB }\end{array}$ & S25A & $3.97 \times 10^{-13}$ & 91.89 \\
\hline 25 & NP_250046.1 & & PA1355 & S29G & $8.22 \times 10^{-13}$ & 0 \\
\hline 26 & NP_252564.1 & narG & $\begin{array}{l}\text { respiratory nitrate } \\
\text { reductase alpha chain }\end{array}$ & A144V & $1.26 \times 10^{-12}$ & 31.02 \\
\hline 27 & NP_249234.1 & & PA0543 & A49T & $1.30 \times 10^{-12}$ & 73.72 \\
\hline 28 & NP_249234.1 & & PA0543 & A38V & $2.12 \times 10^{-12}$ & 73.14 \\
\hline 29 & NP_250447.1 & cysH & $\begin{array}{l}\text { 3'-phosphoadenosine- } 5^{\prime} \text { - } \\
\text { phosphosulfate } \\
\text { reductase }\end{array}$ & L2P & $2.54 \times 10^{-12}$ & 12.54 \\
\hline 30 & NP_249234.1 & & PA0543 & A103T & $2.90 \times 10^{-12}$ & 73.12 \\
\hline 31 & NP_249234.1 & & PA0543 & R53W & $2.96 \times 10^{-12}$ & 72.91 \\
\hline 32 & NP_249234.1 & & PA0543 & L320V & $2.96 \times 10^{-12}$ & 72.91 \\
\hline 33 & NP_249234.1 & & PA0543 & G285A & $2.97 \times 10^{-12}$ & 72.71 \\
\hline 34 & NP_249234.1 & & PA0543 & P35S & $3.26 \times 10^{-12}$ & 72.81 \\
\hline 35 & NP_249234.1 & & PA0543 & $\mathrm{D} 252 \mathrm{~A}$ & $3.27 \times 10^{-12}$ & 72.84 \\
\hline 36 & NP_249234.1 & & PA0543 & S149L & $3.36 \times 10^{-12}$ & 70.02 \\
\hline 37 & NP_249234.1 & & PA0543 & G243S & $3.59 \times 10^{-12}$ & 72.7 \\
\hline 38 & NP_249234.1 & & PA0543 & V152I & $3.99 \times 10^{-12}$ & 72.59 \\
\hline 39 & NP_249234.1 & & PA0543 & $\mathrm{L} 235 \mathrm{P}$ & $4.63 \times 10^{-12}$ & 70.55 \\
\hline 40 & NP_249234.1 & & PA0543 & P34V & $4.81 \times 10^{-12}$ & 72.38 \\
\hline 41 & NP_249234.1 & & PA0543 & V196G & $4.81 \times 10^{-12}$ & 72.38 \\
\hline 42 & NP_252377.1 & $p p c$ & $\begin{array}{l}\text { phosphoenolpyruvate } \\
\text { carboxylase }\end{array}$ & $\mathrm{T} 722 \mathrm{M}$ & $5.56 \times 10^{-12}$ & NA \\
\hline 43 & NP_252377.1 & $p p c$ & $\begin{array}{l}\text { phosphoenolpyruvate } \\
\text { carboxylase }\end{array}$ & A399V & $5.60 \times 10^{-12}$ & NA \\
\hline 44 & NP_252377.1 & $p p c$ & $\begin{array}{l}\text { phosphoenolpyruvate } \\
\text { carboxylase }\end{array}$ & V829S & $5.60 \times 10^{-12}$ & NA \\
\hline 45 & NP_252019.1 & & PA3329 & G86D & $6.19 \times 10^{-12}$ & 46.07 \\
\hline 46 & NP_250181.1 & & $\begin{array}{l}\text { PA1490 probable } \\
\text { transcriptional regulator }\end{array}$ & S30Y & $6.62 \times 10^{-12}$ & 78.34 \\
\hline 47 & NP_250181.1 & & $\begin{array}{l}\text { PA1490 probable } \\
\text { transcriptional regulator }\end{array}$ & S207A & $6.63 \times 10^{-12}$ & 78.37 \\
\hline 48 & NP_249976.1 & & $\begin{array}{l}\text { PA1490 probable } \\
\text { transcriptional regulator }\end{array}$ & D3V & $8.77 \times 10^{-12}$ & 18.67 \\
\hline 49 & NP_250181.1 & & $\begin{array}{l}\text { PA1490 probable } \\
\text { transcriptional regulator }\end{array}$ & R245Q & $9.56 \times 10^{-12}$ & 77.93 \\
\hline 50 & NP_250181.1 & & $\begin{array}{l}\text { PA1490 probable } \\
\text { transcriptional regulator }\end{array}$ & T255A & $9.56 \times 10^{-12}$ & 77.93 \\
\hline 51 & NP_250181.1 & & $\begin{array}{l}\text { PA1490 probable } \\
\text { transcriptional regulator }\end{array}$ & P256A & $9.56 \times 10^{-12}$ & 77.93 \\
\hline 52 & NP_249912.1 & & PA1221 & V11A & $9.72 \times 10^{-12}$ & 11.21 \\
\hline 53 & NP_252019.1 & & PA3329 & $\mathrm{N} 206 \mathrm{H}$ & $1.40 \times 10^{-11}$ & 46.27 \\
\hline 54 & NP_253633.1 & mutL & $\begin{array}{l}\text { DNA mismatch repair } \\
\text { protein MutL }\end{array}$ & A406T & $1.46 \times 10^{-11}$ & 46.27 \\
\hline 55 & NP_250181.1 & & $\begin{array}{l}\text { PA1490 probable } \\
\text { transcriptional regulator }\end{array}$ & E158D & $2.05 \times 10^{-11}$ & 73.04 \\
\hline
\end{tabular}


B. RESULTS

\begin{tabular}{|c|c|c|c|c|c|c|}
\hline Rank & Accession & $\begin{array}{l}\text { Gene } \\
\text { name }\end{array}$ & Protein name & Mutation & p value & $\begin{array}{l}\text { Odds } \\
\text { ratio }\end{array}$ \\
\hline 56 & NP_253633.1 & mutL & $\begin{array}{l}\text { DNA mismatch repair } \\
\text { protein MutL }\end{array}$ & $\begin{array}{l}392 \\
\text { deletion }\end{array}$ & $2.43 \times 10^{-11}$ & 9.09 \\
\hline 57 & NP_253053.1 & $i c i A$ & $\begin{array}{l}\text { inhibitor of chromosome } \\
\text { initiation IciA }\end{array}$ & M1L & $2.51 \times 10^{-11}$ & 11.62 \\
\hline 58 & NP_252377.1 & $p p c$ & $\begin{array}{l}\text { phosphoenolpyruvate } \\
\text { carboxylase }\end{array}$ & E788D & $2.63 \times 10^{-11}$ & NA \\
\hline 59 & NP_253633.1 & mutL & $\begin{array}{l}\text { DNA mismatch repair } \\
\text { protein MutL }\end{array}$ & A391V & $2.76 \times 10^{-11}$ & 9.00 \\
\hline 60 & NP_253616.1 & & PA4929 & C20R & $3.28 \times 10^{-11}$ & NA \\
\hline 61 & NP_254231.1 & & PA5544 & I611V & $3.69 \times 10^{-11}$ & NA \\
\hline 62 & NP_253633.1 & mutL & $\begin{array}{l}\text { DNA mismatch repair } \\
\text { protein MutL }\end{array}$ & S2N & $3.76 \times 10^{-11}$ & 9.01 \\
\hline 63 & NP_253633.1 & mutL & $\begin{array}{l}\text { DNA mismatch repair } \\
\text { protein MutL }\end{array}$ & R207Q & $4.38 \times 10^{-11}$ & 9.10 \\
\hline 64 & NP_249762.1 & braF & $\begin{array}{l}\text { branched-chain amino } \\
\text { acid transport protein } \\
\text { BraF }\end{array}$ & $\mathrm{A} 231 \mathrm{~T}$ & $4.48 \times 10^{-11}$ & 60.51 \\
\hline 65 & NP_253633.1 & mutL & $\begin{array}{l}\text { DNA mismatch repair } \\
\text { protein MutL }\end{array}$ & E428D & $5.00 \times 10^{-11}$ & 8.91 \\
\hline 66 & NP_253108.1 & ftsI & $\begin{array}{l}\text { penicillin-binding protein } \\
3\end{array}$ & $\mathrm{R} 504 \mathrm{C}$ & $5.18 \times 10^{-11}$ & 70.53 \\
\hline 67 & NP_252019.1 & & PA3329 & L66F & $5.34 \times 10^{-11}$ & 39.52 \\
\hline 68 & NP_252019.1 & & PA3329 & T148A & $5.34 \times 10^{-11}$ & 39.52 \\
\hline 69 & NP_252019.1 & & PA3329 & E409G & $5.45 \times 10^{-11}$ & 39.66 \\
\hline 70 & NP_253633.1 & mutL & $\begin{array}{l}\text { DNA mismatch repair } \\
\text { protein MutL }\end{array}$ & T118A & $5.73 \times 10^{-11}$ & 8.94 \\
\hline 71 & NP_249718.1 & $a m a B$ & $\begin{array}{l}\text { delta1-Piperideine-6- } \\
\text { carboxylate } \\
\text { dehydrogenase }\end{array}$ & P45S & $5.74 \times 10^{-11}$ & 15.57 \\
\hline 72 & NP_249718.1 & $a m a B$ & $\begin{array}{l}\text { delta1-Piperideine-6- } \\
\text { carboxylate } \\
\text { dehydrogenase }\end{array}$ & E406A & $5.74 \times 10^{-11}$ & 15.57 \\
\hline 73 & NP_252019.1 & & PA3329 & R75Q & $6.11 \times 10^{-11}$ & 40.98 \\
\hline 74 & NP_252019.1 & & PA3329 & A181S & $6.54 \times 10^{-11}$ & 40.00 \\
\hline 75 & NP_252019.1 & & PA3329 & V71L & $6.99 \times 10^{-11}$ & 40.65 \\
\hline 76 & NP_251234.1 & & PA2544 & T33S & $8.24 \times 10^{-11}$ & 5.24 \\
\hline 77 & NP_249718.1 & $a m a B$ & $\begin{array}{l}\text { delta1-Piperideine-6- } \\
\text { carboxylate } \\
\text { dehydrogenase }\end{array}$ & S14L & $9.72 \times 10^{-11}$ & 15.63 \\
\hline 78 & NP_249718.1 & $a m a B$ & $\begin{array}{l}\text { delta1-Piperideine-6- } \\
\text { carboxylate } \\
\text { dehydrogenase }\end{array}$ & G71R & $9.95 \times 10^{-11}$ & 15.36 \\
\hline 79 & NP_249718.1 & $a m a B$ & $\begin{array}{l}\text { delta1-Piperideine-6- } \\
\text { carboxylate } \\
\text { dehydrogenase }\end{array}$ & $\mathrm{N} 25 \mathrm{H}$ & $1.23 \times 10^{-10}$ & 15.36 \\
\hline
\end{tabular}


B. RESULTS

\begin{tabular}{|c|c|c|c|c|c|c|}
\hline Rank & Accession & $\begin{array}{l}\text { Gene } \\
\text { name }\end{array}$ & Protein name & Mutation & $\mathrm{p}$ value & $\begin{array}{l}\text { Odds } \\
\text { ratio }\end{array}$ \\
\hline 80 & NP_249718.1 & $a m a B$ & $\begin{array}{l}\text { delta1-Piperideine-6- } \\
\text { carboxylate } \\
\text { dehydrogenase }\end{array}$ & R49C & $1.23 \times 10^{-10}$ & 15.36 \\
\hline 81 & NP_249718.1 & $a m a B$ & $\begin{array}{l}\text { delta1-Piperideine-6- } \\
\text { carboxylate } \\
\text { dehydrogenase }\end{array}$ & K340R & $1.23 \times 10^{-10}$ & 15.36 \\
\hline 82 & NP_249976.1 & & $\begin{array}{l}\text { PA1285 probable } \\
\text { transcriptional regulator }\end{array}$ & R5K & $1.24 \times 10^{-10}$ & NA \\
\hline 83 & NP_249718.1 & $a m a B$ & $\begin{array}{l}\text { delta1-Piperideine-6- } \\
\text { carboxylate } \\
\text { dehydrogenase }\end{array}$ & V357I & $1.24 \times 10^{-10}$ & 15.34 \\
\hline 84 & NP_249718.1 & $a m a B$ & $\begin{array}{l}\text { delta1-Piperideine-6- } \\
\text { carboxylate } \\
\text { dehydrogenase }\end{array}$ & Q379E & $1.35 \times 10^{-10}$ & 15.30 \\
\hline 85 & NP_250038.1 & & $\begin{array}{l}\text { PA1347 probable } \\
\text { transcriptional regulator }\end{array}$ & I6T & $1.59 \times 10^{-10}$ & 21.01 \\
\hline 86 & NP_250038.1 & & $\begin{array}{l}\text { PA1347 probable } \\
\text { transcriptional regulator }\end{array}$ & Q30E & $1.59 \times 10^{-10}$ & 21.01 \\
\hline 87 & NP_250038.1 & & $\begin{array}{l}\text { PA1347 probable } \\
\text { transcriptional regulator }\end{array}$ & D97G & $1.87 \times 10^{-10}$ & 20.87 \\
\hline 88 & NP_250038.1 & & $\begin{array}{l}\text { PA1347 probable } \\
\text { transcriptional regulator }\end{array}$ & N40T & $1.99 \times 10^{-10}$ & 20.84 \\
\hline 89 & NP_249718.1 & $a m a B$ & $\begin{array}{l}\text { delta1-Piperideine-6- } \\
\text { carboxylate } \\
\text { dehydrogenase }\end{array}$ & A253D & $2.16 \times 10^{-10}$ & 15.22 \\
\hline 90 & NP_251593.1 & cobJ & $\begin{array}{l}\text { precorrin-3 methylase } \\
\text { CobJ }\end{array}$ & T4A & $2.20 \times 10^{-10}$ & 5.08 \\
\hline 91 & NP_252956.1 & fusA1 & elongation factor $\mathrm{G}$ & D588G & $2.45 \times 10^{-10}$ & NA \\
\hline 92 & NP_251604.1 & & $\begin{array}{l}\text { probable permease of } \\
\text { ABC transporter }\end{array}$ & A281V & $2.46 \times 10^{-10}$ & NA \\
\hline 93 & NP_253552.1 & ureA & urease gamma subunit & E67G & $2.54 \times 10^{-10}$ & NA \\
\hline 94 & NP_251108.1 & & PA2418 & A47T & $3.65 \times 10^{-10}$ & 15.13 \\
\hline 95 & NP_249140.1 & & PA0449 & W38R & $3.75 \times 10^{-10}$ & 26.32 \\
\hline 96 & NP_249140.1 & & PA0449 & G39S & $3.75 \times 10^{-10}$ & 26.32 \\
\hline 97 & NP_252584.1 & & $\begin{array}{l}\text { PA3895 probable } \\
\text { transcriptional regulator }\end{array}$ & P316L & $4.36 \times 10^{-10}$ & 16.44 \\
\hline 98 & NP_249005.1 & & $\begin{array}{l}\text { L-cysteine transporter of } \\
\text { ABC system FliY }\end{array}$ & K139R & $4.39 \times 10^{-10}$ & NA \\
\hline 99 & NP_251108.1 & & PA2418 & W96R & $4.59 \times 10^{-10}$ & 14.80 \\
\hline 100 & NP_253532.1 & $\operatorname{dipZ}$ & $\begin{array}{l}\text { thiol:disulfide } \\
\text { interchange protein DipZ }\end{array}$ & I451V & $5.38 \times 10^{-10}$ & 30.11 \\
\hline
\end{tabular}

\section{B.6. Dataset 2 treeWAS results}


Table 23: SNPs identified with treeWAS from dataset 2, SNPs within core genes. SNPs with absolute simultaneous association scores greater than or equal to 26 are reported.

\begin{tabular}{|c|c|c|c|c|c|c|}
\hline Rank & Accession & $\begin{array}{l}\text { Gene } \\
\text { name }\end{array}$ & Protein name & Mutation & $\begin{array}{c}\text { Association } \\
\text { score }\end{array}$ & p value \\
\hline 1 & NP_250663.1 & $p q q F$ & $\begin{array}{l}\text { pyrroloquinoline quinone } \\
\text { biosynthesis protein } \mathrm{F}\end{array}$ & H3R & 51 & 0 \\
\hline 2 & NP_250663.1 & $p q q F$ & $\begin{array}{l}\text { pyrroloquinoline quinone } \\
\text { biosynthesis protein } \mathrm{F}\end{array}$ & $\mathrm{H} 12 \mathrm{R}$ & 50 & 0 \\
\hline 3 & NP_251980.1 & tle1 & Tle1 & D168E & 45 & 0 \\
\hline 4 & NP_253053.1 & $i c i A$ & $\begin{array}{l}\text { inhibitor of chromosome } \\
\text { initiation IciA }\end{array}$ & M1L & 44 & 0 \\
\hline 5 & NP_253957.1 & & PA5270 & S5A & 43 & 0 \\
\hline 6 & NP_250046.1 & & PA1355 & F33S & 42 & 0 \\
\hline 7 & NP_250046.1 & & PA1355 & C37G & 42 & 0 \\
\hline 8 & NP_251048.1 & & PA2358 & L2P & 42 & 0 \\
\hline 9 & NP_250442.1 & & PA1751 & $\begin{array}{l}1 \\
\text { insertion }\end{array}$ & -41 & 0 \\
\hline 10 & NP_249912.1 & & PA1221 & V11A & 40 & 0 \\
\hline 11 & NP_250447.1 & cysH & $\begin{array}{l}3^{\prime} \text {-phosphoadenosine- } 5 \text { '- } \\
\text { phosphosulfate } \\
\text { reductase }\end{array}$ & L2P & 39 & 0 \\
\hline 12 & NP_248733.1 & & PA0043 & M1D & 38 & 0 \\
\hline 13 & NP_248733.1 & & PA0043 & W4R & 38 & 0 \\
\hline 14 & NP_252820.1 & & $\begin{array}{l}\text { PA4131 probable } \\
\text { iron-sulfur protein }\end{array}$ & $\mathrm{R} 48 \mathrm{P}$ & 37 & 0 \\
\hline 15 & NP_249491.1 & & PA0800 & H78R & 36 & 0 \\
\hline 16 & NP_249538.1 & & diguanylate cyclase & D34G & 36 & 0 \\
\hline 17 & NP_250386.1 & $p s c P$ & $\begin{array}{l}\text { translocation protein in } \\
\text { type III secretion }\end{array}$ & I83T & 34 & 0 \\
\hline 18 & NP_250046.1 & & PA1355 & S29G & 33 & 0 \\
\hline 19 & NP_250737.1 & & $\begin{array}{l}\text { PA2047 probable } \\
\text { transcriptional regulator }\end{array}$ & M1V & 33 & 0 \\
\hline 20 & NP_250737.1 & & $\begin{array}{l}\text { PA2047 probable } \\
\text { transcriptional regulator }\end{array}$ & V3A & 33 & 0 \\
\hline 21 & NP_250737.1 & & $\begin{array}{l}\text { PA2047 probable } \\
\text { transcriptional regulator }\end{array}$ & T6A & 33 & 0 \\
\hline 22 & NP_251234.1 & & PA2544 & G20C & 31 & 0 \\
\hline 23 & NP_251980.1 & tle1 & Tle1 & N131S & 29 & 0 \\
\hline 24 & NP_253462.1 & & PA4774 & $\begin{array}{l}308 \\
\text { deletion }\end{array}$ & -29 & 0 \\
\hline 25 & NP_253462.1 & & PA4774 & X350E & -29 & 0 \\
\hline 26 & NP_250386.1 & $p s c P$ & $\begin{array}{l}\text { translocation protein in } \\
\text { type III secretion }\end{array}$ & L18P & 28 & 0 \\
\hline 27 & NP_251980.1 & tle1 & Tle1 & R92K & 28 & 0 \\
\hline 28 & NP_251980.1 & tle1 & Tle1 & I109F & 28 & 0 \\
\hline 29 & NP_249705.1 & wapB & $\begin{array}{l}\text { 1,2-glucosyltransferase } \\
\text { WapB }\end{array}$ & S25A & 27 & 0 \\
\hline 30 & NP_249976.1 & & $\begin{array}{l}\text { PA1285 probable } \\
\text { transcriptional regulator }\end{array}$ & D3V & 27 & 0 \\
\hline
\end{tabular}




\begin{tabular}{|c|c|c|c|c|c|c|}
\hline Rank & Accession & $\begin{array}{l}\text { Gene } \\
\text { name }\end{array}$ & Protein name & Mutation & $\begin{array}{c}\text { Association } \\
\text { score }\end{array}$ & p value \\
\hline 31 & NP_251980.1 & tle1 & Tle1 & P2S & 27 & 0 \\
\hline 32 & NP_251980.1 & tle1 & Tle1 & $\begin{array}{l}3 \\
\text { insertion }\end{array}$ & 27 & 0 \\
\hline 33 & NP_251980.1 & tle1 & Tle1 & F6V & 27 & 0 \\
\hline 34 & NP_251980.1 & tle1 & Tle1 & I8V & 27 & 0 \\
\hline 35 & NP_251980.1 & tle1 & Tle1 & T19P & 27 & 0 \\
\hline 36 & NP_251980.1 & tle1 & Tle1 & Y20L & 27 & 0 \\
\hline 37 & NP_251980.1 & tle1 & Tle1 & V53E & 27 & 0 \\
\hline 38 & NP_251980.1 & tle1 & Tle1 & A62T & 27 & 0 \\
\hline 39 & NP_251980.1 & tle1 & Tle1 & I63L & 27 & 0 \\
\hline 40 & NP_251980.1 & tle1 & Tle1 & $\mathrm{I} 65 \mathrm{~V}$ & 27 & 0 \\
\hline 41 & NP_251980.1 & tle1 & Tle1 & $\mathrm{I} 94 \mathrm{~F}$ & 27 & 0 \\
\hline 42 & NP_251980.1 & tle1 & Tle1 & D102S & 27 & 0 \\
\hline 43 & NP_251980.1 & tle1 & Tle1 & K111R & 27 & 0 \\
\hline 44 & NP_251980.1 & tle1 & Tle1 & F127Y & 27 & 0 \\
\hline 45 & NP_252321.1 & & PA3631 & $\begin{array}{l}377 \\
\text { deletion }\end{array}$ & -27 & 0 \\
\hline 46 & NP_252321.1 & & PA3631 & X409L & -27 & 0 \\
\hline 47 & NP_253781.1 & & $\begin{array}{l}\text { PA5094 probable } \\
\text { ATP-binding component } \\
\text { of ABC transporter }\end{array}$ & $\begin{array}{l}269 \\
\text { deletion }\end{array}$ & -27 & 0 \\
\hline 48 & NP_253781.1 & & $\begin{array}{l}\text { PA5094 probable } \\
\text { ATP-binding component } \\
\text { of ABC transporter }\end{array}$ & X277Q & -27 & 0 \\
\hline 49 & NP_251980.1 & tle1 & Tle1 & V149T & 26 & 0 \\
\hline 50 & NP_251980.1 & tle1 & Tle1 & N3E & 26 & 0 \\
\hline 51 & NP_251980.1 & tle1 & Tle1 & $\mathrm{F} 4 \mathrm{~V}$ & 26 & 0 \\
\hline 52 & NP_251980.1 & tle1 & Tle1 & G5T & 26 & 0 \\
\hline 53 & NP_251980.1 & tle1 & Tle1 & H7S & 26 & 0 \\
\hline 54 & NP_251980.1 & tle1 & Tle1 & T11E & 26 & 0 \\
\hline 55 & NP_251980.1 & tle1 & Tle1 & $\mathrm{H} 12 \mathrm{~F}$ & 26 & 0 \\
\hline 56 & NP_251980.1 & tle1 & Tle1 & V14K & 26 & 0 \\
\hline 57 & NP_251980.1 & tle1 & Tle1 & A15E & 26 & 0 \\
\hline 58 & NP_251980.1 & tle1 & Tle1 & G16R & 26 & 0 \\
\hline 59 & NP_251980.1 & tle1 & Tle1 & R17L & 26 & 0 \\
\hline 60 & NP_251980.1 & tle1 & Tle1 & D21E & 26 & 0 \\
\hline 61 & NP_251980.1 & tle1 & Tle1 & S22P & 26 & 0 \\
\hline 62 & NP_251980.1 & tle1 & Tle1 & K23S & 26 & 0 \\
\hline 63 & NP_251980.1 & tle1 & Tle1 & $\mathrm{K} 24 \mathrm{~V}$ & 26 & 0 \\
\hline 64 & NP_251980.1 & tle1 & Tle1 & E27K & 26 & 0 \\
\hline 65 & NP_251980.1 & tle1 & Tle1 & L30S & 26 & 0 \\
\hline 66 & NP_251980.1 & tle1 & Tle1 & K31R & 26 & 0 \\
\hline 67 & NP_251980.1 & tle1 & Tle1 & Q33V & 26 & 0 \\
\hline 68 & NP_251980.1 & tle1 & Tle1 & D35E & 26 & 0 \\
\hline 69 & NP_251980.1 & tle1 & Tle1 & V38K & 26 & 0 \\
\hline 70 & NP_251980.1 & tle1 & Tle1 & F39Y & 26 & 0 \\
\hline 71 & NP_251980.1 & tle1 & Tle1 & S40N & 26 & 0 \\
\hline 72 & NP_251980.1 & tle1 & Tle1 & V54S & 26 & 0 \\
\hline
\end{tabular}


B. RESULTS

\begin{tabular}{|c|c|c|c|c|c|c|}
\hline Rank & Accession & $\begin{array}{l}\text { Gene } \\
\text { name }\end{array}$ & Protein name & Mutation & $\begin{array}{c}\text { Association } \\
\text { score }\end{array}$ & $\mathrm{p}$ value \\
\hline 73 & NP_251980.1 & tle1 & Tle1 & G55S & 26 & $\overline{0}$ \\
\hline 74 & NP_251980.1 & tle1 & Tle1 & A57I & 26 & 0 \\
\hline 75 & NP_251980.1 & tle1 & Tle1 & T66S & 26 & 0 \\
\hline 76 & NP_251980.1 & tle1 & Tle1 & L67I & 26 & 0 \\
\hline 77 & NP_251980.1 & tle1 & Tle1 & F69Y & 26 & 0 \\
\hline 78 & NP_251980.1 & tle1 & Tle1 & K77R & 26 & 0 \\
\hline 79 & NP_251980.1 & tle1 & Tle1 & V83L & 26 & 0 \\
\hline 80 & NP_251980.1 & tle1 & Tle1 & S84P & 26 & 0 \\
\hline 81 & NP_251980.1 & tle1 & Tle1 & C87R & 26 & 0 \\
\hline 82 & NP_251980.1 & tle1 & Tle1 & $\mathrm{I} 98 \mathrm{~V}$ & 26 & 0 \\
\hline 83 & NP_251980.1 & tle1 & Tle1 & S100E & 26 & 0 \\
\hline 84 & NP_251980.1 & tle1 & Tle1 & D103V & 26 & 0 \\
\hline 85 & NP_251980.1 & tle1 & Tle1 & I104Q & 26 & 0 \\
\hline 86 & NP_251980.1 & tle1 & Tle1 & S106G & 26 & 0 \\
\hline 87 & NP_251980.1 & tle1 & Tle1 & R107L & 26 & 0 \\
\hline 88 & NP_251980.1 & tle1 & Tle1 & K125R & 26 & 0 \\
\hline 89 & NP_251980.1 & tle1 & Tle1 & M132L & 26 & 0 \\
\hline 90 & NP_251980.1 & tle1 & Tle1 & G136A & 26 & 0 \\
\hline 91 & NP_251980.1 & tle1 & Tle1 & E138A & 26 & 0 \\
\hline 92 & NP_251980.1 & tle1 & Tle1 & I144V & 26 & 0 \\
\hline 93 & NP_251980.1 & tle1 & Tle1 & L148I & 26 & 0 \\
\hline 94 & NP_251980.1 & tle1 & Tle1 & Q150R & 26 & 0 \\
\hline 95 & NP_251980.1 & tle1 & Tle1 & A154V & 26 & 0 \\
\hline 96 & NP_251980.1 & tle1 & Tle1 & F156Q & 26 & 0 \\
\hline 97 & NP_251980.1 & tle1 & Tle1 & Y157G & 26 & 0 \\
\hline 98 & NP_251980.1 & tle1 & Tle1 & L160T & 26 & 0 \\
\hline 99 & NP_251980.1 & tle1 & Tle1 & $\begin{array}{l}160 \\
\text { insertion }\end{array}$ & 26 & 0 \\
\hline 100 & NP_251980.1 & tle1 & Tle1 & K161E & 26 & 0 \\
\hline 101 & NP_251980.1 & tle1 & Tle1 & R163P & 26 & 0 \\
\hline 102 & NP_251980.1 & tle1 & Tle1 & K165L & 26 & 0 \\
\hline 103 & NP_251980.1 & tle1 & Tle1 & N167D & 26 & 0 \\
\hline 104 & NP_251980.1 & tle1 & Tle1 & V169S & 26 & 0 \\
\hline 105 & NP_251980.1 & tle1 & Tle1 & G171T & 26 & 0 \\
\hline
\end{tabular}

\section{B.7. Dataset 2 random forest results}

Table 24: SNPs identified with RF from dataset 2, SNPs within core genes. SNPs with importances greater than or equal to 0.002 are reported.

\begin{tabular}{llllll}
\hline Rank & Accession & Gene name & Protein name & Mutation & Importance \\
\hline 1 & NP_250663.1 & $p q q F$ & $\begin{array}{l}\text { pyrroloquinoline quinone } \\
\text { biosynthesis protein F } \\
\text { pyrroloquinoline quinone } \\
\text { biosynthesis protein F }\end{array}$ & H12R & 0.14 \\
2 & NP_250663.1 & $p q q F$ & $\begin{array}{l}\text { PA5438 probable transcriptional } \\
\text { regulator }\end{array}$ & $\begin{array}{l}272 \\
\text { deletion }\end{array}$ & 0.098 \\
3 & NP_254125.1 & & 0.076 \\
\hline
\end{tabular}




\begin{tabular}{|c|c|c|c|c|c|}
\hline Rank & Accession & Gene name & Protein name & Mutation & Importance \\
\hline 4 & NP_251831.1 & $w b p M$ & $\begin{array}{l}\text { nucleotide sugar } \\
\text { epimerase/dehydratase } \mathrm{WbpM}\end{array}$ & V113P & 0.049 \\
\hline 5 & NP_248708.1 & fimt & methionyl-tRNA formyltransferase & I196V & 0.04 \\
\hline 6 & NP_250663.1 & $p q q F$ & $\begin{array}{l}\text { pyrroloquinoline quinone } \\
\text { biosynthesis protein } \mathrm{F}\end{array}$ & K92R & 0.039 \\
\hline 7 & NP_249976.1 & & $\begin{array}{l}\text { PA1285 probable transcriptional } \\
\text { regulator }\end{array}$ & D3V & 0.015 \\
\hline 8 & NP_253053.1 & $i c i A$ & $\begin{array}{l}\text { inhibitor of chromosome initiation } \\
\text { IciA }\end{array}$ & M1L & 0.012 \\
\hline 9 & NP_250447.1 & cysH & $\begin{array}{l}\text { 3'-phosphoadenosine-5'- } \\
\text { phosphosulfate } \\
\text { reductase }\end{array}$ & L2P & 0.012 \\
\hline 10 & NP_248733.1 & & PA0043 & W4R & 0.01 \\
\hline 11 & NP_249939.1 & aprF & $\begin{array}{l}\text { Alkaline protease secretion outer } \\
\text { membrane protein AprF precursor }\end{array}$ & T82A & 0.009 \\
\hline 12 & NP_250442.1 & & 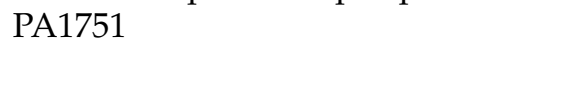 & $\begin{array}{l}1 \\
\text { insertion }\end{array}$ & 0.009 \\
\hline 13 & NP_251249.1 & & PA2559 & A161S & 0.008 \\
\hline 14 & NP_250319.1 & & $\begin{array}{l}\text { PA1628 probable } \\
\text { 3-hydroxyacyl-CoA dehydrogenase }\end{array}$ & R48Q & 0.008 \\
\hline 15 & NP_249004.1 & & $\begin{array}{l}\text { L-cysteine transporter of } \mathrm{ABC} \\
\text { system YecS }\end{array}$ & $\begin{array}{l}164 \\
\text { insertion }\end{array}$ & 0.008 \\
\hline 16 & NP_251435.1 & & PA2745 probable hydrolase & L88G & 0.007 \\
\hline 17 & NP_250108.1 & & PA1417 probable decarboxylase & T262A & 0.007 \\
\hline 18 & NP_249286.1 & $\operatorname{lptD}$ & LPS-assembly protein LptD & E812K & 0.007 \\
\hline 19 & NP_251993.1 & & $\begin{array}{l}\text { PA3303 probable major facilitator } \\
\text { superfamily (MFS) transporter }\end{array}$ & H262R & 0.006 \\
\hline 20 & NP_250663.1 & $p q q F$ & $\begin{array}{l}\text { pyrroloquinoline quinone } \\
\text { biosynthesis protein } \mathrm{F}\end{array}$ & C240G & 0.006 \\
\hline 21 & NP_250310.1 & & $\begin{array}{l}\text { PA1619 probable transcriptional } \\
\text { regulator }\end{array}$ & $\mathrm{I} 14 \mathrm{~V}$ & 0.005 \\
\hline 22 & NP_251980.1 & tle1 & Tle1 & $\mathrm{I} 109 \mathrm{~F}$ & 0.005 \\
\hline 23 & NP_251358 & & PA2668 & $\begin{array}{l}5 \\
\text { insertion }\end{array}$ & 0.005 \\
\hline 24 & NP_251232.1 & & PA2542 & $\begin{array}{l}1130 \\
\text { insertion }\end{array}$ & 0.004 \\
\hline 25 & NP_251315.1 & & PA2625 & V84I & 0.004 \\
\hline 26 & NP_249533.1 & & $\begin{array}{l}\text { PA0842 probable glycosyl } \\
\text { transferase }\end{array}$ & K251R & 0.004 \\
\hline 27 & NP_252886.1 & $b f i S$ & BfiS & $\mathrm{E} 431 \mathrm{~A}$ & 0.004 \\
\hline 28 & NP_249622.1 & pirA & ferric enterobactin receptor PirA & A370T & 0.004 \\
\hline 29 & NP_249286.1 & $\operatorname{lptD}$ & LPS-assembly protein LptD & D593N & 0.004 \\
\hline 30 & NP_252466.1 & $x$ seA & $\begin{array}{l}\text { exodeoxyribonuclease VII large } \\
\text { subunit }\end{array}$ & L325Q & 0.004 \\
\hline 31 & NP_253191.1 & opdD & $\begin{array}{l}\text { Glycine-glutamate dipeptide porin } \\
\text { OpdP }\end{array}$ & E30A & 0.004 \\
\hline 32 & NP_248937.1 & & $\begin{array}{l}\text { PA0246 probable major facilitator } \\
\text { superfamily (MFS) transporter }\end{array}$ & Y43F & 0.004 \\
\hline 33 & NP_251716.1 & & PA3026 & E464Q & 0.004 \\
\hline
\end{tabular}




\begin{tabular}{|c|c|c|c|c|c|}
\hline Rank & Accession & Gene name & Protein name & Mutation & Importance \\
\hline 34 & NP_251210.1 & $c z c A$ & $\begin{array}{l}\text { Resistance-Nodulation-Cell } \\
\text { Division (RND) divalent metal } \\
\text { cation efflux transporter CzcA }\end{array}$ & R595K & 0.004 \\
\hline 35 & NP_253957.1 & & PA5270 & S5A & 0.004 \\
\hline 36 & NP_248783.1 & tse 6 & Tse6 & $\begin{array}{l}387 \\
\text { deletion }\end{array}$ & 0.004 \\
\hline 37 & NP_248891.1 & & PA0200 & E64D & 0.003 \\
\hline 38 & NP_253549.1 & & $\begin{array}{l}\text { PA4862 probable ATP-binding } \\
\text { component of ABC transporter }\end{array}$ & $\mathrm{A} 174 \mathrm{~T}$ & 0.003 \\
\hline 39 & NP_251601.1 & & $\begin{array}{l}\text { PA2911 probable TonB-dependent } \\
\text { receptor }\end{array}$ & Y684D & 0.003 \\
\hline 40 & NP_248783.1 & tse 6 & Tse6 & A392L & 0.003 \\
\hline 41 & NP_253939.1 & & $\begin{array}{l}\text { PA5252 probable ATP-binding } \\
\text { component of ABC transporter }\end{array}$ & T225I & 0.003 \\
\hline 42 & NP_253462.1 & & PA4774 & $\mathrm{X} 350 \mathrm{E}$ & 0.003 \\
\hline 43 & NP_252350.1 & & $\begin{array}{l}\text { PA3660 probable } \\
\text { sodium/hydrogen antiporter }\end{array}$ & A14T & 0.003 \\
\hline 44 & NP_248969.1 & & PA0278 & V111A & 0.003 \\
\hline 45 & NP_253191.1 & opdD & $\begin{array}{l}\text { Glycine-glutamate dipeptide porin } \\
\text { OpdP }\end{array}$ & N31T & 0.003 \\
\hline 46 & NP_252017.1 & & $\begin{array}{l}\text { PA3327 probable non-ribosomal } \\
\text { peptide synthetase }\end{array}$ & E402K & 0.003 \\
\hline 47 & NP_251604.1 & & $\begin{array}{l}\text { PA2914 probable permease of } \mathrm{ABC} \\
\text { transporter }\end{array}$ & $\mathrm{I} 255 \mathrm{~V}$ & 0.003 \\
\hline 48 & NP_251115.1 & $p v d G$ & PvdG & D178E & 0.003 \\
\hline 49 & NP_251358 & & PA2668 & R4L & 0.003 \\
\hline 50 & NP_249925.1 & & PA1234 & N39D & 0.003 \\
\hline 51 & NP_254006.1 & $\mathrm{radC}$ & DNA repair protein RadC & D212G & 0.003 \\
\hline 52 & NP_250687.1 & & $\begin{array}{l}\text { PA1997 probable AMP-binding } \\
\text { enzyme }\end{array}$ & A328T & 0.003 \\
\hline 53 & NP_251593.1 & cobJ & precorrin-3 methylase CobJ & $\mathrm{T} 4 \mathrm{~A}$ & 0.003 \\
\hline 54 & NP_248764.1 & $p p k A$ & $\begin{array}{l}\text { serine/threonine protein kinase } \\
\text { PpkA }\end{array}$ & L295M & 0.003 \\
\hline 55 & NP_253663.1 & $\operatorname{aruH}$ & $\begin{array}{l}\text { Arginine:Pyruvate Transaminas, } \\
\text { AruH }\end{array}$ & R84Q & 0.003 \\
\hline 56 & NP_252007.1 & & PA3317 & S126N & 0.003 \\
\hline 57 & NP_249290.1 & & PA0599 & A20T & 0.002 \\
\hline 58 & NP_250415.1 & psck & type III export protein PscK & E137D & 0.002 \\
\hline 59 & NP_253413.1 & $\operatorname{cbr} A$ & two-component sensor CbrA & T16A & 0.002 \\
\hline 60 & NP_250612.1 & & $\begin{array}{l}\text { PA1922 probable TonB-dependent } \\
\text { receptor }\end{array}$ & V419A & 0.002 \\
\hline 61 & NP_250774.1 & & $\begin{array}{l}\text { PA2084 probable asparagine } \\
\text { synthetase }\end{array}$ & V118A & 0.002 \\
\hline 62 & NP_251003 & & PA2313 & $\mathrm{H} 205 \mathrm{O}$ & 0.002 \\
\hline 63 & NP_250806.1 & & PA2116 & T148A & 0.002 \\
\hline 64 & NP_250939.1 & $b k d B$ & $\begin{array}{l}\text { branched-chain alpha-keto acid } \\
\text { dehydrogenase (lipoamide } \\
\text { component) }\end{array}$ & S321G & 0.002 \\
\hline 65 & NP_251749.1 & pelF & PelF & N229S & 0.002 \\
\hline
\end{tabular}


B. RESULTS

\begin{tabular}{|c|c|c|c|c|c|}
\hline Rank & Accession & Gene name & Protein name & Mutation & Importance \\
\hline 66 & NP_251519.1 & & PA2829 & G15A & 0.002 \\
\hline 67 & NP_250692.1 & & PA2002 & V457G & 0.002 \\
\hline 68 & NP_251005.1 & & PA2315 & V284I & 0.002 \\
\hline 69 & NP_249124.1 & & PA0433 & H116Q & 0.002 \\
\hline 70 & NP_249633.1 & & $\begin{array}{l}\text { PA0942 probable transcriptional } \\
\text { regulator }\end{array}$ & D172A & 0.002 \\
\hline 71 & NP_254011.1 & $\operatorname{sph} R$ & $\begin{array}{l}\text { Sphingosine-responsive Regulator, } \\
\text { SphR }\end{array}$ & D347E & 0.002 \\
\hline 72 & NP_251124.1 & & PA2434 & A145T & 0.002 \\
\hline 73 & NP_251005.1 & & PA2315 & L306V & 0.002 \\
\hline 74 & NP_250470.1 & & assimilatory nitrate reductase & T549S & 0.002 \\
\hline 75 & NP_251358 & & PA2668 & R4I & 0.002 \\
\hline 76 & NP_251157.1 & fox $R$ & Anti-sigma factor FoxR & A28G & 0.002 \\
\hline 77 & NP_252126.1 & & PA3436 & H92R & 0.002 \\
\hline 78 & NP_253777.1 & vgrG5 & VgrG5 & A242T & 0.002 \\
\hline 79 & NP_253179.1 & $\operatorname{mag} D$ & MagD & M1009V & 0.002 \\
\hline 80 & NP_251602.1 & & $\begin{array}{l}\text { PA2912 probable ATP-binding } \\
\text { component of ABC transporter }\end{array}$ & S185G & 0.002 \\
\hline 81 & NP_249292.1 & $\operatorname{agt} R$ & $\begin{array}{l}\text { two-component response regulator, } \\
\text { AgtR }\end{array}$ & $\begin{array}{l}1 \\
\text { deletion }\end{array}$ & 0.002 \\
\hline 82 & NP_249158.1 & & PA0467 & G140A & 0.002 \\
\hline 83 & NP_251114.1 & $p v d L$ & PvdL & V3938I & 0.002 \\
\hline 84 & YP_008719781.1 & & PA4638 & F87R & 0.002 \\
\hline 85 & NP_253669 & & probable two-component sensor & N904S & 0.002 \\
\hline 86 & NP_250356.1 & fha2 & Fha2 & G277S & 0.002 \\
\hline 87 & NP_250705.1 & $\operatorname{liu} A$ & $\begin{array}{l}\text { putative isovaleryl-CoA } \\
\text { dehydrogenase }\end{array}$ & A179P & 0.002 \\
\hline 88 & NP_249019.1 & $a a a A$ & $\begin{array}{l}\text { arginine-specific autotransporter of } \\
\text { Pseudomonas aeruginosa, AaaA }\end{array}$ & H516R & 0.002 \\
\hline 89 & NP_250023.1 & & PA1332 & C48R & 0.002 \\
\hline 90 & NP_250630.1 & & PA1940 & T284A & 0.002 \\
\hline 91 & NP_252454.1 & & PA3765 & S62R & 0.002 \\
\hline 92 & NP_252030.1 & & PA3340 & S181N & 0.002 \\
\hline 93 & NP_253231.1 & $\operatorname{lep} A$ & $\begin{array}{l}\text { Pseudomonas aeruginosa-derived } \\
\text { large extracellular protease, LepA }\end{array}$ & S550N & 0.002 \\
\hline 94 & NP_251970.1 & oprO & $\begin{array}{l}\text { Pyrophosphate-specific outer } \\
\text { membrane porin OprO precursor }\end{array}$ & F86Y & 0.002 \\
\hline 95 & NP_251813.1 & & PA3123 & S37T & 0.002 \\
\hline 96 & NP_251980.1 & tle1 & Tle1 & N131S & 0.002 \\
\hline 97 & NP_249399.1 & & $\begin{array}{l}\text { PA0708 probable transcriptional } \\
\text { regulator }\end{array}$ & G181S & 0.002 \\
\hline 98 & NP_249104.1 & $\operatorname{chp} A$ & $\begin{array}{l}\text { component of chemotactic signal } \\
\text { transduction system }\end{array}$ & V594L & 0.002 \\
\hline 99 & NP_249104.1 & $\operatorname{chp} A$ & $\begin{array}{l}\text { component of chemotactic signal } \\
\text { transduction system }\end{array}$ & E825A & 0.002 \\
\hline 100 & NP_252913.1 & & $\begin{array}{l}\text { PA4223 probable ATP-binding } \\
\text { component of ABC transporter }\end{array}$ & S484R & 0.002 \\
\hline 101 & NP_252236.1 & $\operatorname{alg} X$ & alginate biosynthesis protein $\mathrm{Alg} X$ & E201D & 0.002 \\
\hline 102 & NP_251766.1 & & PA3076 & D42A & 0.002 \\
\hline
\end{tabular}


B. RESULTS

\begin{tabular}{llllll}
\hline Rank & Accession & Gene name & Protein name & Mutation & Importance \\
\hline 103 & NP_251204.1 & antC & anthranilate dioxygenase reductase & D87N & 0.002 \\
104 & NP_249109.1 & & PA0418 & T455R & 0.002 \\
\hline
\end{tabular}

B.8. Dataset 2 support vector machine results

Table 25: SNPs identified with SVM from dataset 2, SNPs within core genes. Top 100 SNPs with absolute hyperplane coefficients greater than 0.03 are reported.

\begin{tabular}{|c|c|c|c|c|c|}
\hline Rank & Accession & Gene name & Protein name & Mutation & $\begin{array}{l}\text { Hyperplane } \\
\text { coefficient }\end{array}$ \\
\hline 1 & NP_251083.1 & & PA2393 putative dipeptidase & G22A & 0.5017 \\
\hline 2 & NP_251083.1 & & PA2393 putative dipeptidase & $\begin{array}{l}21 \\
\text { insertion }\end{array}$ & -0.5011 \\
\hline 3 & NP_251083.1 & & PA2393 putative dipeptidase & G22V & -0.5008 \\
\hline 4 & NP_251083.1 & & PA2393 putative dipeptidase & $\begin{array}{l}22 \\
\text { insertion }\end{array}$ & 0.4963 \\
\hline 5 & NP_249622.1 & pirA & ferric enterobactin receptor PirA & A370T & -0.3394 \\
\hline 6 & NP_252366.1 & $\operatorname{mexK}$ & MexK & E765A & 0.3369 \\
\hline 7 & NP_252366.1 & $\operatorname{mexK}$ & MexK & K694R & -0.2909 \\
\hline 8 & NP_249622.1 & pirA & ferric enterobactin receptor PirA & A228V & 0.2810 \\
\hline 9 & NP_249622.1 & pirA & ferric enterobactin receptor PirA & V128A & 0.2808 \\
\hline 10 & NP_253633.1 & mutL & $\begin{array}{l}\text { DNA mismatch repair protein } \\
\text { MutL }\end{array}$ & E428D & -0.2808 \\
\hline 11 & NP_252612.1 & & PA3923 & A137V & 0.2803 \\
\hline 12 & NP_251391.1 & & $\begin{array}{l}\text { PA2701 probable major facilitator } \\
\text { superfamily (MFS) transporter }\end{array}$ & V304A & 0.2802 \\
\hline 13 & NP_250322.1 & & $\begin{array}{l}\text { PA1631 probable acyl-CoA } \\
\text { dehydrogenase }\end{array}$ & $\mathrm{D} 172 \mathrm{~N}$ & -0.2801 \\
\hline 14 & NP_251747.1 & & PA3057 & A72R & 0.2572 \\
\hline 15 & NP_252156.1 & & $\begin{array}{l}\text { PA3466 probable ATP-dependent } \\
\text { RNA helicase }\end{array}$ & $\mathrm{S} 4 \mathrm{~F}$ & 0.2422 \\
\hline 16 & NP_251831.1 & $w b p M$ & $\begin{array}{l}\text { nucleotide sugar } \\
\text { epimerase/dehydratase WbpM }\end{array}$ & I7G & 0.1939 \\
\hline 17 & NP_251831.1 & $w b p M$ & $\begin{array}{l}\text { nucleotide sugar } \\
\text { epimerase/dehydratase } \mathrm{WbpM}\end{array}$ & K8R & 0.1863 \\
\hline 18 & NP_251858.1 & gyrA & DNA gyrase subunit A & D87Y & -0.1763 \\
\hline 19 & NP_249064.1 & fts $Y$ & $\begin{array}{l}\text { signal recognition particle receptor } \\
\text { FtsY }\end{array}$ & $\begin{array}{l}120 \\
\text { deletion }\end{array}$ & -0.1527 \\
\hline 20 & NP_249004.1 & & $\begin{array}{l}\text { L-cysteine transporter of ABC } \\
\text { system YecS }\end{array}$ & $\begin{array}{l}164 \\
\text { insertion }\end{array}$ & 0.1507 \\
\hline 21 & NP_250804.1 & & $\begin{array}{l}\text { PA2114 probable major facilitator } \\
\text { superfamily (MFS) transporter }\end{array}$ & A168T & 0.1165 \\
\hline 22 & NP_249593.1 & & PA0902 & G162D & 0.1158 \\
\hline 23 & NP_253213.1 & & PA4523 & A184P & 0.1158 \\
\hline 24 & NP_249979.1 & & $\begin{array}{l}\text { PA1288 probable outer membrane } \\
\text { protein precursor }\end{array}$ & G176S & -0.1142 \\
\hline 25 & NP_248764.1 & ppkA & $\begin{array}{l}\text { serine/threonine protein kinase } \\
\text { PpkA }\end{array}$ & Q105L & 0.1140 \\
\hline
\end{tabular}


B. RESULTS

\begin{tabular}{|c|c|c|c|c|c|}
\hline Rank & Accession & Gene name & Protein name & Mutation & $\begin{array}{l}\text { Hyperplane } \\
\text { coefficient }\end{array}$ \\
\hline 26 & NP_254125.1 & & $\begin{array}{l}\text { PA5438 probable transcriptional } \\
\text { regulator }\end{array}$ & $\begin{array}{l}272 \\
\text { deletion }\end{array}$ & 0.1026 \\
\hline 27 & NP_250890.1 & & $\begin{array}{l}\text { Cyclic-guanylate-specific } \\
\text { phosphodiesterase }\end{array}$ & A529T & -0.0962 \\
\hline 28 & NP_248764.1 & ppkA & $\begin{array}{l}\text { serine/threonine protein kinase } \\
\text { PpkA }\end{array}$ & L295M & 0.0941 \\
\hline 29 & NP_250036.1 & & PA1345 & L17P & 0.0905 \\
\hline 30 & NP_249860.1 & & PA1169 probable lipoxygenase & T137A & 0.0851 \\
\hline 31 & NP_248764.1 & ppkA & $\begin{array}{l}\text { serine/threonine protein kinase } \\
\text { PpkA }\end{array}$ & A108V & 0.0846 \\
\hline 32 & NP_253847.1 & & drug efflux transporter & V211I & -0.0789 \\
\hline 33 & NP_253847.1 & & drug efflux transporter & $\mathrm{H} 421 \mathrm{~N}$ & -0.0785 \\
\hline 34 & NP_249860.1 & & PA1169 probable lipoxygenase & I181L & 0.0775 \\
\hline 35 & NP_249860.1 & & PA1169 probable lipoxygenase & R203G & 0.0774 \\
\hline 36 & NP_251367.1 & & $\begin{array}{l}\text { PA2677 probable type II secretion } \\
\text { protein }\end{array}$ & M12V & 0.0765 \\
\hline 37 & NP_249860.1 & & PA1169 probable lipoxygenase & G84A & 0.0737 \\
\hline 38 & NP_253847.1 & & drug efflux transporter & T86A & -0.0734 \\
\hline 39 & NP_252321.1 & & РA3631 & $\begin{array}{l}377 \\
\text { deletion }\end{array}$ & -0.0702 \\
\hline 40 & NP_252321.1 & & PA3631 & X409L & -0.0698 \\
\hline 41 & NP_250765.1 & & PA2075 & Q427L & -0.0696 \\
\hline 42 & NP_253462.1 & & PA4774 & $\begin{array}{l}308 \\
\text { deletion }\end{array}$ & -0.0673 \\
\hline 43 & NP_253462.1 & & PA4774 & X350E & -0.0673 \\
\hline 44 & NP_251367.1 & & $\begin{array}{l}\text { PA2677 probable type II secretion } \\
\text { protein }\end{array}$ & P10L & -0.0668 \\
\hline 45 & NP_249109.1 & & PA0418 & T8P & -0.0664 \\
\hline 46 & NP_249064.1 & $f t s Y$ & $\begin{array}{l}\text { signal recognition particle receptor } \\
\text { FtsY }\end{array}$ & $\begin{array}{l}121 \\
\text { deletion }\end{array}$ & 0.0662 \\
\hline 47 & NP_251980.1 & tle1 & Tle1 & D168E & 0.0611 \\
\hline 48 & NP_253373.1 & & PA4684 & $\begin{array}{l}407 \\
\text { deletion }\end{array}$ & -0.0610 \\
\hline 49 & NP_253373.1 & & PA4684 & X433L & -0.0610 \\
\hline 50 & NP_253053.1 & iciA & $\begin{array}{l}\text { inhibitor of chromosome initiation } \\
\text { IciA }\end{array}$ & M1L & 0.0606 \\
\hline 51 & NP_253651.1 & $\operatorname{parC}$ & topoisomerase IV subunit A & S87L & -0.0599 \\
\hline 52 & NP_249988.1 & & PÁ1297 probable metal transporter & $\begin{array}{l}172 \\
\text { insertion }\end{array}$ & -0.0599 \\
\hline 53 & NP_251124.1 & & PA2434 & A145T & -0.0595 \\
\hline 54 & NP_251522.1 & tpm & thiopurine methyltransferase & H82R & 0.0590 \\
\hline 55 & NP_250442.1 & & 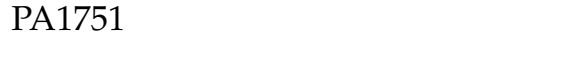 & $\begin{array}{l}1 \\
\text { insertion }\end{array}$ & -0.0584 \\
\hline 56 & NP_250447.1 & cysH & $\begin{array}{l}3^{\prime} \text {-phosphoadenosine- } 5^{\prime} \text { - } \\
\text { phosphosulfate } \\
\text { reductase }\end{array}$ & L2P & 0.0584 \\
\hline 57 & NP_252681.1 & sltB3 & SltB3 & $\mathrm{H} 240 \mathrm{Y}$ & -0.0564 \\
\hline 58 & NP_251048.1 & & PA2358 & $\mathrm{L} 2 \mathrm{P}$ & 0.0556 \\
\hline
\end{tabular}


B. RESULTS

\begin{tabular}{|c|c|c|c|c|c|}
\hline Rank & Accession & Gene name & Protein name & Mutation & $\begin{array}{l}\text { Hyperplane } \\
\text { coefficient }\end{array}$ \\
\hline 59 & NP_250755.1 & $\overline{p c o A}$ & $\begin{array}{l}\text { copper resistance protein A } \\
\text { precursor }\end{array}$ & V401M & -0.0551 \\
\hline 60 & NP_253284.1 & & $\begin{array}{l}\text { PA4594 probable ATP-binding } \\
\text { component of ABC transporter }\end{array}$ & G203S & -0.0550 \\
\hline 61 & NP_253231.1 & lepA & $\begin{array}{l}\text { Pseudomonas aeruginosa-derived } \\
\text { large extracellular protease, LepA }\end{array}$ & N424S & 0.0547 \\
\hline 62 & NP_248840.1 & & anti-sigma factor & E12D & 0.0543 \\
\hline 63 & NP_250124.1 & & PA1433 & M476L & 0.0542 \\
\hline 64 & NP_251519.1 & & PA2829 & G15A & -0.0540 \\
\hline 65 & NP_249538.1 & & diguanylate cyclase & D34G & 0.0534 \\
\hline 66 & NP_248783.1 & tse6 & Tse6 & $\begin{array}{l}387 \\
\text { deletion }\end{array}$ & -0.0532 \\
\hline 67 & NP_248783.1 & tse6 & Tse6 & A392L & -0.0532 \\
\hline 68 & NP_250025.1 & & PA1334 probable oxidoreductase & T7A & -0.0532 \\
\hline 69 & NP_248783.1 & tse 6 & Tse6 & R388L & 0.0529 \\
\hline 70 & NP_248783.1 & tse6 & Tse6 & $\begin{array}{l}388 \\
\text { deletion }\end{array}$ & 0.0529 \\
\hline 71 & NP_251351.1 & & PA2661 & $\mathrm{D} 255 \mathrm{O}$ & 0.0524 \\
\hline 72 & NP_251100.1 & fpo $F$ & FpvF & I276V & -0.0523 \\
\hline 73 & NP_251052.1 & $\operatorname{dotU3}$ & DotU3 & X253A & 0.0522 \\
\hline 74 & NP_250046.1 & & PA1355 & F33S & 0.0521 \\
\hline 75 & NP_249696.1 & & PA1005 & M14I & 0.0521 \\
\hline 76 & NP_250056.1 & & $\begin{array}{l}\text { PA1365 probable siderophore } \\
\text { receptor }\end{array}$ & P799L & 0.0521 \\
\hline 77 & NP_251980.1 & tle1 & Tle1 & $\mathrm{I} 231 \mathrm{~V}$ & 0.0521 \\
\hline 78 & NP_250046.1 & & PA1355 & C37G & 0.0520 \\
\hline 79 & NP_251063.1 & vgrG3 & VgrG3 & T46S & 0.0518 \\
\hline 80 & NP_252454.1 & & PA3765 & S62R & -0.0515 \\
\hline 81 & NP_252538.1 & & PA3849 & A52T & 0.0514 \\
\hline 82 & NP_252538.1 & & PA3849 & E44A & 0.0514 \\
\hline 83 & NP_249838.1 & & $\begin{array}{l}\text { PA1147 probable amino acid } \\
\text { permease }\end{array}$ & V396A & -0.0511 \\
\hline 84 & NP_249020.1 & & PA0329 & N62S & -0.0511 \\
\hline 85 & NP_253377.1 & hitB & $\begin{array}{l}\text { iron (III)-transport system } \\
\text { permease HitB }\end{array}$ & L345V & -0.0510 \\
\hline 86 & NP_250613.1 & & PA1923 & E65D & -0.0510 \\
\hline 87 & NP_250126.1 & & $\begin{array}{l}\text { PA1435 probable } \\
\text { Resistance-Nodulation-Cell } \\
\text { Division (RND) efflux membrane } \\
\text { fusion protein precursor }\end{array}$ & L230P & 0.0505 \\
\hline 88 & NP_250557.1 & & PA1866 & G425A & 0.0504 \\
\hline 89 & NP_250923.1 & $p s l C$ & PslC & D173G & -0.0503 \\
\hline 90 & NP_253960.1 & & PA5273 & G233O & 0.0503 \\
\hline 91 & NP_249976.1 & & $\begin{array}{l}\text { PA1285 probable transcriptional } \\
\text { regulator }\end{array}$ & D3V & 0.0501 \\
\hline 92 & NP_064724.1 & gyrB & DNA gyrase subunit B & $\mathrm{S} 466 \mathrm{~F}$ & 0.0498 \\
\hline 93 & NP_252235.1 & $\operatorname{alg} G$ & $\begin{array}{l}\text { alginate-c5-mannuronan- } \\
\text { epimerase } \\
\text { AlgG }\end{array}$ & G58S & 0.0496 \\
\hline
\end{tabular}




\section{B. RESULTS}

\begin{tabular}{|c|c|c|c|c|c|}
\hline Rank & Accession & Gene name & Protein name & Mutation & $\begin{array}{l}\text { Hyperplane } \\
\text { coefficient }\end{array}$ \\
\hline 94 & NP_253284.1 & & $\begin{array}{l}\text { PA4594 probable ATP-binding } \\
\text { component of ABC transporter }\end{array}$ & Q5R & -0.0496 \\
\hline 95 & NP_251257.1 & & PA2567 & G487S & -0.0496 \\
\hline 96 & NP_249912.1 & & PA1221 & V11A & 0.0494 \\
\hline 97 & NP_250290.1 & & $\begin{array}{l}\text { PA1599 probable transcriptional } \\
\text { regulator }\end{array}$ & D37E & -0.0493 \\
\hline 98 & NP_250127.1 & & $\begin{array}{l}\text { PA1436 probable } \\
\text { Resistance-Nodulation-Cell }\end{array}$ & T93S & 0.0492 \\
\hline 99 & NP_253170.1 & mreC & $\begin{array}{l}\text { Division (RND) efflux transporter } \\
\text { rod shape-determining protein } \\
\text { MreC }\end{array}$ & S296O & 0.0492 \\
\hline 100 & NP_250126.1 & & $\begin{array}{l}\text { PA1435 probable } \\
\text { Resistance-Nodulation-Cell } \\
\text { Division (RND) efflux membrane } \\
\text { fusion protein precursor }\end{array}$ & D325A & 0.0490 \\
\hline
\end{tabular}

\section{B.9. Dataset 3 PLINK results}

Table 26: Genes and SNPs identified with PLINK from dataset 3, combined gene presence/absence and SNPs within core genes. Top 100 variants with $p$ values less than $1 \times 10^{-8}$ are reported. 2640 genes had $p$ values less than $1 \times 10^{-8}$.

\begin{tabular}{|c|c|c|c|c|c|c|}
\hline Rank & Accession & $\begin{array}{l}\text { Gene } \\
\text { name }\end{array}$ & Protein name & Mutation & $\mathrm{p}$ value & $\begin{array}{l}\text { Odds } \\
\text { ratio }\end{array}$ \\
\hline 1 & - & & $\begin{array}{l}\text { G5 domain containing } \\
\text { protein }\end{array}$ & & $2.677 \times 10^{-60}$ & 24.43 \\
\hline 2 & NP_252650.1 & & $\begin{array}{l}\text { PA3961 probable } \\
\text { ATP-dependent helicase }\end{array}$ & A8S & $1.59 \times 10^{-49}$ & 0.09 \\
\hline 3 & NP_252650.1 & & $\begin{array}{l}\text { PA3961 probable } \\
\text { ATP-dependent helicase }\end{array}$ & E214D & $1.59 \times 10^{-49}$ & 0.09 \\
\hline 4 & NP_252777.1 & & $\begin{array}{l}\text { PA4088 probable } \\
\text { aminotransferase }\end{array}$ & L208P & $3.663 \times 10^{-49}$ & 9.21 \\
\hline 5 & NP_253497.1 & $f d h E$ & FdhE protein & A235T & $1.793 \times 10^{-45}$ & 10.55 \\
\hline 6 & NP_254099.1 & & PA5412 & $\mathrm{A} 68 \mathrm{~V}$ & $2.795 \times 10^{-44}$ & 11.09 \\
\hline 7 & NP_250624.1 & & PA1934 & S99T & $5.353 \times 10^{-44}$ & 12.06 \\
\hline 8 & NP_252274.1 & $g l p D$ & $\begin{array}{l}\text { glycerol-3-phosphate } \\
\text { dehydrogenase }\end{array}$ & $\mathrm{R} 274 \mathrm{H}$ & $8.968 \times 10^{-44}$ & 0.10 \\
\hline 9 & NP_252276.1 & & $\begin{array}{l}\text { PA3586probable } \\
\text { hydrolase }\end{array}$ & T102A & $8.968 \times 10^{-44}$ & 0.10 \\
\hline 10 & NP_252172.1 & met $G$ & $\begin{array}{l}\text { methionyl-tRNA } \\
\text { synthetase }\end{array}$ & V76A & $3.895 \times 10^{-40}$ & 0.11 \\
\hline 11 & NP_252172.1 & met $G$ & $\begin{array}{l}\text { methionyl-tRNA } \\
\text { synthetase }\end{array}$ & E659Q & $3.895 \times 10^{-40}$ & 0.11 \\
\hline 12 & NP_251087.1 & $p v d E$ & $\begin{array}{l}\text { pyoverdine biosynthesis } \\
\text { protein PvdE }\end{array}$ & E327S & $5.119 \times 10^{-40}$ & 0.11 \\
\hline 13 & NP_251087.1 & $p v d E$ & $\begin{array}{l}\text { pyoverdine biosynthesis } \\
\text { protein PvdE }\end{array}$ & S394E & $5.119 \times 10^{-40}$ & 0.11 \\
\hline
\end{tabular}


B. RESULTS

\begin{tabular}{|c|c|c|c|c|c|c|}
\hline Rank & Accession & $\begin{array}{l}\text { Gene } \\
\text { name }\end{array}$ & Protein name & Mutation & $\mathrm{p}$ value & $\begin{array}{l}\text { Odds } \\
\text { ratio }\end{array}$ \\
\hline 14 & NP_253584.1 & & TonB-dependent receptor & L14P & $3.371 \times 10^{-39}$ & 2077 \\
\hline 15 & NP_251968.1 & & PA3278 & A99V & $3.106 \times 10^{-37}$ & NA \\
\hline 16 & NP_252100.1 & hasI & HasI & $\mathrm{R} 112 \mathrm{H}$ & $1.871 \times 10^{-36}$ & NA \\
\hline 17 & - & & hypothetical protein & & $2.769 \times 10^{-36}$ & 30.01 \\
\hline 18 & _ & & hypothetical protein & & $6.071 \times 10^{-36}$ & 157.2 \\
\hline 19 & NP_252053.1 & $\operatorname{amiR}$ & $\begin{array}{l}\text { aliphatic amidase } \\
\text { regulator }\end{array}$ & D163N & $1.827 \times 10^{-35}$ & 2011 \\
\hline 20 & - & & terminase & & $4.339 \times 10^{-35}$ & 19.38 \\
\hline 21 & - & & hypothetical protein & & $1.081 \times 10^{-34}$ & 21.71 \\
\hline 22 & NP_253011.1 & & PA4321 r & & $9.14 \times 10^{-34}$ & 0.13 \\
\hline 23 & NP_253481.1 & & PA4793 & $\mathrm{R} 84 \mathrm{G}$ & $1.119 \times 10^{-33}$ & 7.16 \\
\hline 24 & NP_254235.1 & & $\begin{array}{l}\text { PA5548 probable major } \\
\text { facilitator superfamily } \\
\text { (MFS) transporter }\end{array}$ & V27I & $6.548 \times 10^{-33}$ & 169.20 \\
\hline 25 & NP_253584.1 & & TonB-dependent receptor & D269N & $1.476 \times 10^{-32}$ & NA \\
\hline 26 & NP_249248.1 & & PA0557 & Q176K & $3.887 \times 10^{-32}$ & 0.18 \\
\hline 27 & - & & methyltransferase & & $1.478 \times 10^{-31}$ & 39.57 \\
\hline 28 & NP_250726.1 & & PA2036 & & $4.727 \times 10^{-31}$ & 24.37 \\
\hline 29 & NP_253058.1 & & PA4368 & L145M & $4.823 \times 10^{-31}$ & 8.27 \\
\hline 30 & NP_249286.1 & $\operatorname{lptD}$ & $\begin{array}{l}\text { LPS-assembly protein } \\
\text { LptD }\end{array}$ & I888V & $8.87 \times 10^{-31}$ & 10.07 \\
\hline 31 & NP_248957.1 & davT & $\begin{array}{l}\text { delta-aminovalerate } \\
\text { aminotransferase }\end{array}$ & $\mathrm{A} 289 \mathrm{~V}$ & $1.016 \times 10^{-30}$ & 110.20 \\
\hline 32 & NP_253508.1 & & $\begin{array}{l}\text { PA4821 probable } \\
\text { transporter }\end{array}$ & Q408K & $5.19 \times 10^{-30}$ & 16.46 \\
\hline 33 & - & & hypothetical protein & & $8.94 \times 10^{-30}$ & 25.28 \\
\hline 34 & NP_253921.1 & & $\begin{array}{l}\text { PA5234 probable } \\
\text { oxidoreductase }\end{array}$ & $\mathrm{I} 30 \mathrm{~V}$ & $2.442 \times 10^{-29}$ & 30.44 \\
\hline 35 & NP_252875.1 & & PA4186 & & $3.38 \times 10^{-29}$ & 85.36 \\
\hline 36 & NP_252110.1 & & $\begin{array}{l}\text { PA3420 probable } \\
\text { transcriptional regulator }\end{array}$ & T193A & $5.076 \times 10^{-29}$ & 14.51 \\
\hline 37 & NP_253408.1 & $\operatorname{trm} A$ & $\begin{array}{l}\text { tRNA (uracil-5-)- } \\
\text { methyltransferase }\end{array}$ & S292N & $1.783 \times 10^{-28}$ & 21.64 \\
\hline 38 & NP_251442.1 & & PA2752 & $\mathrm{R} 36 \mathrm{H}$ & $6.795 \times 10^{-28}$ & 0.07 \\
\hline 39 & NP_248783.1 & tse 6 & Tse6 & F422Y & $9.084 \times 10^{-28}$ & 47.61 \\
\hline 40 & NP_249248.1 & & PA0557 & P19L & $2.338 \times 10^{-27}$ & 0.21 \\
\hline 41 & NP_250087.1 & & $\begin{array}{l}\text { PA1396 probable } \\
\text { two-component sensor }\end{array}$ & D509N & $3.426 \times 10^{-27}$ & 10.96 \\
\hline 42 & - & & $\begin{array}{l}\text { type I-E } \\
\text { CRISPR-associated } \\
\text { protein Cas5/CasD }\end{array}$ & & $1.165 \times 10^{-26}$ & 18.91 \\
\hline 43 & NP_250086.1 & & PA1395 & G16S & $1.932 \times 10^{-26}$ & 9.15 \\
\hline 44 & NP_250699.1 & $h m g A$ & $\begin{array}{l}\text { homogentisate } \\
\text { 1,2-dioxygenase }\end{array}$ & A250G & $2.086 \times 10^{-26}$ & 5.31 \\
\hline 45 & NP_249709.1 & & PA1018 & E159K & $2.353 \times 10^{-26}$ & 18.26 \\
\hline 46 & NP_253660.1 & thiC & $\begin{array}{l}\text { thiamin biosynthesis } \\
\text { protein ThiC }\end{array}$ & D447N & $2.413 \times 10^{-26}$ & 20.06 \\
\hline
\end{tabular}




\begin{tabular}{|c|c|c|c|c|c|c|}
\hline Rank & Accession & $\begin{array}{l}\text { Gene } \\
\text { name }\end{array}$ & Protein name & Mutation & $\mathrm{p}$ value & $\begin{array}{l}\text { Odds } \\
\text { ratio }\end{array}$ \\
\hline 47 & NP_252283.1 & & $\begin{array}{l}\text { PA3593 probable } \\
\text { acyl-CoA dehydrogenase }\end{array}$ & P451S & $4.82 \times 10^{-26}$ & 27.32 \\
\hline 48 & NP_254178.1 & & $\begin{array}{l}\text { PA5491 probable } \\
\text { cytochrome }\end{array}$ & T67S & $7.193 \times 10^{-26}$ & 14.55 \\
\hline 49 & NP_252534.1 & & $\begin{array}{l}\text { PA3845 probable } \\
\text { transcriptional regulator }\end{array}$ & $\mathrm{A} 50 \mathrm{~T}$ & $7.288 \times 10^{-26}$ & 27.23 \\
\hline 50 & NP_253888.1 & & PA5201 & D137N & $1.131 \times 10^{-25}$ & 11.29 \\
\hline 51 & NP_253148.1 & & PA4458 & & $1.194 \times 10^{-25}$ & 18.06 \\
\hline 52 & - & & $\begin{array}{l}\text { elongation factor } \mathrm{Tu} \\
\text { (pseudogene) }\end{array}$ & & $2.797 \times 10^{-25}$ & 5.17 \\
\hline 53 & _ & & hypothetical protein & & $4.752 \times 10^{-25}$ & 93.00 \\
\hline 54 & - & & protein kinase & & $4.752 \times 10^{-25}$ & 93.00 \\
\hline 55 & - & & hypothetical protein & & $1.178 \times 10^{-24}$ & 1414 \\
\hline 56 & NP_251264.1 & alkB1 & alkane-1-monooxygenase & V325A & $1.719 \times 10^{-24}$ & 32.01 \\
\hline 57 & NP_253151.1 & $\operatorname{lptB}$ & $\begin{array}{l}\text { Lipopolysaccharide } \\
\text { export system } \\
\text { ATP-binding protein LptB }\end{array}$ & & $2.772 \times 10^{-24}$ & NA \\
\hline 58 & NP_251706.1 & & PA3016 & & $3.144 \times 10^{-24}$ & 6.14 \\
\hline 59 & - & & $\begin{array}{l}\text { usher CupC3 } \\
\text { (pseudogene) }\end{array}$ & & $3.576 \times 10^{-24}$ & 0.21 \\
\hline 60 & NP_254209.1 & pauA6 & $\begin{array}{l}\text { Glutamylpolyamine } \\
\text { synthetase }\end{array}$ & Q226V & $6.795 \times 10^{-24}$ & NA \\
\hline 61 & NP_248913.1 & & PA0222 & $\mathrm{Y} 8 \mathrm{C}$ & $1.224 \times 10^{-23}$ & 24.70 \\
\hline 62 & NP_253551.1 & ureD & urease accessory protein & A37S & $2.997 \times 10^{-23}$ & NA \\
\hline 63 & NP_248982.1 & oprE & $\begin{array}{l}\text { Anaerobically-induced } \\
\text { outer membrane porin } \\
\text { OprE precursor }\end{array}$ & $\begin{array}{l}440 \\
\text { insertion }\end{array}$ & $5.95 \times 10^{-23}$ & 12.11 \\
\hline 64 & NP_251512.1 & & PA2822 & I120L & $8.727 \times 10^{-23}$ & 11.69 \\
\hline 65 & NP_253413.1 & $\operatorname{cbr} A$ & $\begin{array}{l}\text { two-component sensor } \\
\text { CbrA }\end{array}$ & V673A & $9.505 \times 10^{-23}$ & 57.97 \\
\hline 66 & NP_253590.1 & & $\begin{array}{l}\text { PA4903 probable major } \\
\text { facilitator superfamily } \\
\text { (MFS) transporter }\end{array}$ & $\mathrm{R} 220 \mathrm{H}$ & $1.085 \times 10^{-22}$ & 58.68 \\
\hline 67 & NP_249693.1 & phnB & $\begin{array}{l}\text { anthranilate synthase } \\
\text { component II }\end{array}$ & A73V & $1.237 \times 10^{-22}$ & NA \\
\hline 68 & NP_250047.1 & & PA1356 & & $1.237 \times 10^{-22}$ & NA \\
\hline 69 & NP_251873.1 & $z w f$ & $\begin{array}{l}\text { glucose-6-phosphate } \\
\text { 1-dehydrogenase }\end{array}$ & E109D & $1.237 \times 10^{-22}$ & NA \\
\hline 70 & NP_252214.1 & gloA1 & lactoylglutathione lyase & D87N & $1.237 \times 10^{-22}$ & NA \\
\hline 71 & NP_252769.1 & & $\begin{array}{l}\text { PA } 4080 \text { probable } \\
\text { response regulator }\end{array}$ & N149T & $1.237 \times 10^{-22}$ & NA \\
\hline 72 & - & & hypothetical protein & & $1.237 \times 10^{-22}$ & NA \\
\hline 73 & NP_253616.1 & & PA4929 & A402S & $1.425 \times 10^{-22}$ & 11.52 \\
\hline 74 & NP_251114.1 & $p v d L$ & PvdL & R1655Q & $1.49 \times 10^{-22}$ & NA \\
\hline 75 & NP_248708.1 & fmt & $\begin{array}{l}\text { methionyl-tRNA } \\
\text { formyltransferase }\end{array}$ & A188V & $1.805 \times 10^{-22}$ & 78.48 \\
\hline 76 & NP_249401.1 & gloA2 & lactoylglutathione lyase & A89D & $2.105 \times 10^{-22}$ & 7.79 \\
\hline
\end{tabular}




\begin{tabular}{|c|c|c|c|c|c|c|}
\hline Rank & Accession & $\begin{array}{l}\text { Gene } \\
\text { name }\end{array}$ & Protein name & Mutation & $\mathrm{p}$ value & $\begin{array}{l}\text { Odds } \\
\text { ratio }\end{array}$ \\
\hline 77 & NP_251316.1 & trmU & tRNA methyltransferase & & $3.785 \times 10^{-22}$ & 11.3 \\
\hline 78 & NP_253584.1 & & TonB-dependent receptor & M497T & $4.648 \times 10^{-22}$ & 108.2 \\
\hline 79 & NP_253541.1 & purH & $\begin{array}{l}\text { phosphoribosylaminoimida } \\
\text { formyltransferase }\end{array}$ & 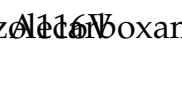 & aidde23 $\times 10^{-22}$ & NA \\
\hline 80 & NP_252516.1 & $\operatorname{lptG}$ & $\begin{array}{l}\text { Lipopolysaccharide } \\
\text { export system permease } \\
\text { protein LptG }\end{array}$ & $\begin{array}{l}218 \\
\text { insertion }\end{array}$ & $5.502 \times 10^{-22}$ & 100.10 \\
\hline 81 & NP_253113.1 & & PA4423 & E219D & $6.694 \times 10^{-22}$ & 11.20 \\
\hline 82 & NP_250443.1 & & PA1752 & Y295H & $7.166 \times 10^{-22}$ & 12.28 \\
\hline 83 & NP_253127.1 & & PA4437 & A67S & $7.308 \times 10^{-22}$ & 24.73 \\
\hline 84 & NP_251939.1 & & $\begin{array}{l}\text { PA3249 probable } \\
\text { transcriptional regulator }\end{array}$ & & $1.341 \times 10^{-21}$ & 22.50 \\
\hline 85 & NP_250885.1 & $h c n C$ & $\begin{array}{l}\text { hydrogen cyanide } \\
\text { synthase HcnC }\end{array}$ & P209L & $1.35 \times 10^{-21}$ & 32.45 \\
\hline 86 & NP_250865.1 & & PA2175 & R91W & $2.189 \times 10^{-21}$ & 64.66 \\
\hline 87 & NP_252485.1 & & PA3796 & $\begin{array}{l}102 \\
\text { insertion }\end{array}$ & $2.313 \times 10^{-21}$ & 9.11 \\
\hline 88 & NP_253669 & & $\begin{array}{l}\text { PA4982 probable } \\
\text { two-component sensor }\end{array}$ & A $825 \mathrm{~V}$ & $2.314 \times 10^{-21}$ & 11.21 \\
\hline 89 & NP_253135.1 & & PA4445 & A54V & $2.519 \times 10^{-21}$ & NA \\
\hline 90 & NP_251911.1 & $\operatorname{csa} A$ & CsaA protein & V34I & $2.644 \times 10^{-21}$ & 11.68 \\
\hline 91 & NP_253403.1 & & $\begin{array}{l}\text { PA4715 probable } \\
\text { aminotransferase }\end{array}$ & A339T & $2.844 \times 10^{-21}$ & 7.18 \\
\hline 92 & NP_252623.1 & & PA3934 & E156A & $3.797 \times 10^{-21}$ & 16.65 \\
\hline 93 & NP_252472.1 & & PA3783 & R68L & $3.948 \times 10^{-21}$ & NA \\
\hline 94 & NP_252438.1 & & $\begin{array}{l}\text { PA3749 probable major } \\
\text { facilitator superfamily } \\
\text { (MFS) transporter }\end{array}$ & & $4.551 \times 10^{-21}$ & 18.45 \\
\hline 95 & NP_253423.1 & & PA4735 & A299V & $7.268 \times 10^{-21}$ & 15.58 \\
\hline 96 & NP_253397.1 & phus & PhuS & Q133H & $7.275 \times 10^{-21}$ & 1207 \\
\hline 97 & NP_251076.1 & $p v d A$ & L-ornithine N5-oxygenase & E369D & $1.12 \times 10^{-20}$ & 20.79 \\
\hline 98 & NP_253584.1 & & TonB-dependent receptor & Q742R & $1.136 \times 10^{-20}$ & 9.00 \\
\hline 99 & NP_251948.1 & & PA3258 & G285S & $1.29 \times 10^{-20}$ & 70.55 \\
\hline 100 & NP_249248.1 & & PA0557 & H191Q & $1.494 \times 10^{-20}$ & NA \\
\hline
\end{tabular}

B.10. Dataset 3 treeWAS results

Table 27: Genes and SNPs identified with treeWAS from dataset 3, combined gene presence/absence and SNPs within core genes. Variants with absolute simultaneous association scores greater than or equal to 26 are reported.

\begin{tabular}{llllrrr}
\hline Rank & Accession & $\begin{array}{l}\text { Gene } \\
\text { name }\end{array}$ & Protein name & Mutation & $\begin{array}{r}\text { Association } \\
\text { score }\end{array}$ & p value \\
\hline 1 & NP_250663.1 & $p q q F$ & $\begin{array}{l}\text { pyrroloquinoline quinone } \\
\text { biosynthesis protein F }\end{array}$ & H3R & 50.5 & 0 \\
2 & NP_250018.1 & & PA1327 probable protease & & -50 & 0 \\
\hline
\end{tabular}


B. RESULTS

\begin{tabular}{|c|c|c|c|c|c|c|}
\hline Rank & Accession & $\begin{array}{l}\text { Gene } \\
\text { name }\end{array}$ & Protein name & Mutation & $\begin{array}{c}\text { Association } \\
\text { score }\end{array}$ & p value \\
\hline 3 & NP_250663.1 & $p q q F$ & $\begin{array}{l}\text { pyrroloquinoline quinone } \\
\text { biosynthesis protein F }\end{array}$ & H12R & 49.5 & 0 \\
\hline 4 & NP_249374.1 & $h x c Y$ & $\mathrm{HxcY}$ & & -49 & 0 \\
\hline 5 & NP_250230.1 & & PA1539 & & -48 & 0 \\
\hline 6 & NP_250420.1 & & PA1729 & & -48 & 0 \\
\hline 7 & NP_249865.1 & nap A & $\begin{array}{l}\text { periplasmic nitrate } \\
\text { reductase protein NapA }\end{array}$ & & -46 & 0 \\
\hline 8 & NP_250376.1 & mas $A$ & enolase-phosphatase E-1 & & -46 & 0 \\
\hline 9 & NP_251980.1 & tle1 & Tle1 & D168E & 45 & 0 \\
\hline 10 & NP_251618.1 & & PA2928 & & -45 & 0 \\
\hline 11 & NP_253053.1 & $i c i A$ & $\begin{array}{l}\text { inhibitor of chromosome } \\
\text { initiation IciA }\end{array}$ & M1L & 44 & 0 \\
\hline 12 & NP_248888.1 & tonB2 & TonB2 & & -44 & 0 \\
\hline 13 & NP_253957.1 & & PA5270 & S5A & 43 & 0 \\
\hline 14 & NP_250046.1 & & PA1355 & F33S & 42 & 0 \\
\hline 15 & NP_250046.1 & & PA1355 & C37G & 42 & 0 \\
\hline 16 & NP_251048.1 & & PA2358 & L2P & 42 & 0 \\
\hline 17 & NP_064723.1 & $\mathrm{rec} F$ & RecF protein & & -42 & 0 \\
\hline 18 & NP_250442.1 & & PA1751 & $\begin{array}{l}1 \\
\text { insertion }\end{array}$ & -41 & 0 \\
\hline 19 & NP_249912.1 & & PA1221 & V11A & 40 & 0 \\
\hline 20 & - & & hypothetical protein & & 40 & 0 \\
\hline 21 & NP_249603.1 & & PA0912 & & -40 & 0 \\
\hline 22 & NP_250447.1 & cysH & $\begin{array}{l}3^{\prime} \text {-phosphoadenosine- } 5^{\prime} \text { - } \\
\text { phosphosulfate } \\
\text { reductase }\end{array}$ & L2P & 39 & 0 \\
\hline 23 & NP_248733.1 & & PA0043 & M1D & 38 & 0 \\
\hline 24 & NP_248733.1 & & PA0043 & W4R & 38 & 0 \\
\hline 25 & NP_252820.1 & & $\begin{array}{l}\text { PA4131 probable } \\
\text { iron-sulfur protein }\end{array}$ & R48P & 37 & 0 \\
\hline 26 & NP_249491.1 & & PA0800 & H78R & 36 & 0 \\
\hline 27 & NP_249538.1 & & diguanylate cyclase & D34G & 36 & 0 \\
\hline 28 & - & & hypothetical protein & & 35 & 0 \\
\hline 29 & NP_251800.1 & & PA3110 & & -35 & 0 \\
\hline 30 & NP_253724.1 & & PA5037 & & -35 & 0 \\
\hline 31 & NP_250386.1 & $p s c P$ & $\begin{array}{l}\text { translocation protein in } \\
\text { type III secretion }\end{array}$ & I83T & 34 & 0 \\
\hline 32 & NP_250046.1 & & PA1355 & S29G & 33 & 0 \\
\hline 33 & NP_250737.1 & & $\begin{array}{l}\text { PA2047 probable } \\
\text { transcriptional regulator }\end{array}$ & M1V & 33 & 0 \\
\hline 34 & NP_250737.1 & & $\begin{array}{l}\text { PA2047 probable } \\
\text { transcriptional regulator }\end{array}$ & V3A & 33 & 0 \\
\hline 35 & NP_250737.1 & & $\begin{array}{l}\text { PA2047 probable } \\
\text { transcriptional regulator }\end{array}$ & T6A & 33 & 0 \\
\hline 36 & NP_250764.1 & & PA2074 & & 33 & 0 \\
\hline 37 & NP_251981.1 & tli1 & Tli1 & & 32 & 0 \\
\hline 38 & NP_251234.1 & & PA2544 & G20C & 31 & 0 \\
\hline 39 & - & & hypothetical protein & & 31 & 0 \\
\hline
\end{tabular}




\begin{tabular}{|c|c|c|c|c|c|c|}
\hline Rank & Accession & $\begin{array}{l}\text { Gene } \\
\text { name }\end{array}$ & Protein name & Mutation & $\begin{array}{c}\text { Association } \\
\text { score }\end{array}$ & $\mathrm{p}$ value \\
\hline 40 & - & & $\begin{array}{l}\text { putative rubredoxin } \\
\text { reductase }\end{array}$ & & 31 & 0 \\
\hline 41 & & & hypothetical protein & & 30 & 0 \\
\hline 42 & NP_251980.1 & tle1 & Tle1 & N131S & 29 & 0 \\
\hline 43 & - & & glyA & & 29 & 0 \\
\hline 44 & - & & $\begin{array}{l}\text { ABC transporter } \\
\text { substrate-binding protein }\end{array}$ & & 29 & 0 \\
\hline 45 & NP_253462.1 & & PA4774 & $\begin{array}{l}308 \\
\text { deletion }\end{array}$ & -28.5 & 0 \\
\hline 46 & NP_253462.1 & & PA4774 & X350E & -28.5 & 0 \\
\hline 47 & NP_250386.1 & $p s c P$ & $\begin{array}{l}\text { translocation protein in } \\
\text { type III secretion }\end{array}$ & L18P & 28 & 0 \\
\hline 48 & NP_251980.1 & tle1 & Tle1 & R92K & 28 & 0 \\
\hline 49 & NP_251980.1 & tle1 & Tle1 & $\mathrm{I} 109 \mathrm{~F}$ & 28 & 0 \\
\hline 50 & - & & $\begin{array}{l}\text { mammalian cell entry } \\
\text { protein (pseudogene) }\end{array}$ & & 28 & 0 \\
\hline 51 & - & & hypothetical protein & & 28 & 0 \\
\hline 52 & _- & & hypothetical protein & & 28 & 0 \\
\hline 53 & - & & $\begin{array}{l}\text { hypothetical protein } \\
\text { (pseudogene) }\end{array}$ & & 28 & 0 \\
\hline 54 & NP_249705.1 & wapB & $\begin{array}{l}\text { 1,2-glucosyltransferase } \\
\text { WapB }\end{array}$ & S25A & 27 & 0 \\
\hline 55 & NP_249976.1 & & $\begin{array}{l}\text { PA1285 probable } \\
\text { transcriptional regulator }\end{array}$ & D3V & 27 & 0 \\
\hline 56 & NP_251980.1 & tle1 & Tle1 & $\mathrm{P} 2 \mathrm{~S}$ & 27 & 0 \\
\hline 57 & NP_251980.1 & tle1 & Tle1 & $\begin{array}{l}3 \\
\text { insertion }\end{array}$ & 27 & 0 \\
\hline 58 & NP_251980.1 & tle1 & Tle1 & F6V & 27 & 0 \\
\hline 59 & NP_251980.1 & tle1 & Tle1 & I8V & 27 & 0 \\
\hline 60 & NP_251980.1 & tle1 & Tle1 & T19P & 27 & 0 \\
\hline 61 & NP_251980.1 & tle1 & Tle1 & Y20L & 27 & 0 \\
\hline 62 & NP_251980.1 & tle1 & Tle1 & V53E & 27 & 0 \\
\hline 63 & NP_251980.1 & tle1 & Tle1 & $\mathrm{A} 62 \mathrm{~T}$ & 27 & 0 \\
\hline 64 & NP_251980.1 & tle1 & Tle1 & I63L & 27 & 0 \\
\hline 65 & NP_251980.1 & tle1 & Tle1 & I65V & 27 & 0 \\
\hline 66 & NP_251980.1 & tle1 & Tle1 & $\mathrm{I} 94 \mathrm{~F}$ & 27 & 0 \\
\hline 67 & NP_251980.1 & tle1 & Tle1 & D102S & 27 & 0 \\
\hline 68 & NP_251980.1 & tle1 & Tle1 & K111R & 27 & 0 \\
\hline 69 & NP_251980.1 & tle1 & Tle1 & F127Y & 27 & 0 \\
\hline 70 & NP_253781.1 & & $\begin{array}{l}\text { PA5094 probable } \\
\text { ATP-binding component } \\
\text { of ABC transporter }\end{array}$ & $\begin{array}{l}269 \\
\text { deletion }\end{array}$ & -27 & 0 \\
\hline 71 & NP_253781.1 & & $\begin{array}{l}\text { PA5094 probable } \\
\text { ATP-binding component } \\
\text { of ABC transporter }\end{array}$ & X277Q & -27 & 0 \\
\hline 72 & - & & hypothetical protein & & 27 & 0 \\
\hline 73 & NP_248731.1 & & $\begin{array}{l}\text { PA0041 probable } \\
\text { hemagglutinin }\end{array}$ & & -27 & 0 \\
\hline
\end{tabular}


B. RESULTS

\begin{tabular}{|c|c|c|c|c|c|c|}
\hline Rank & Accession & $\begin{array}{l}\text { Gene } \\
\text { name }\end{array}$ & Protein name & Mutation & $\begin{array}{c}\text { Association } \\
\text { score }\end{array}$ & p value \\
\hline 74 & NP 250105.1 & & PA1414 & & 27 & 0 \\
\hline 75 & NP_250632.1 & & PA1942 & & 27 & 0 \\
\hline 76 & NP_252321.1 & & PA3631 & $\begin{array}{l}377 \\
\text { deletion }\end{array}$ & -26.5 & 0 \\
\hline 77 & NP_252321.1 & & PA3631 & X409L & -26.5 & 0 \\
\hline 78 & NP_251980.1 & tle1 & Tle1 & V149T & 26 & 0 \\
\hline 79 & NP_251980.1 & tle1 & Tle1 & N3E & 26 & 0 \\
\hline 80 & NP_251980.1 & tle1 & Tle1 & F4V & 26 & 0 \\
\hline 81 & NP_251980.1 & tle1 & Tle1 & G5T & 26 & 0 \\
\hline 82 & NP_251980.1 & tle1 & Tle1 & H7S & 26 & 0 \\
\hline 83 & NP_251980.1 & tle1 & Tle1 & T11E & 26 & 0 \\
\hline 84 & NP_251980.1 & tle1 & Tle1 & $\mathrm{H} 12 \mathrm{~F}$ & 26 & 0 \\
\hline 85 & NP_251980.1 & tle1 & Tle1 & V14K & 26 & 0 \\
\hline 86 & NP_251980.1 & tle1 & Tle1 & A15E & 26 & 0 \\
\hline 87 & NP_251980.1 & tle1 & Tle1 & G16R & 26 & 0 \\
\hline 88 & NP_251980.1 & tle1 & Tle1 & R17L & 26 & 0 \\
\hline 89 & NP_251980.1 & tle1 & Tle1 & $\mathrm{D} 21 \mathrm{E}$ & 26 & 0 \\
\hline 90 & NP_251980.1 & tle1 & Tle1 & S22P & 26 & 0 \\
\hline 91 & NP_251980.1 & tle1 & Tle1 & K23S & 26 & 0 \\
\hline 92 & NP_251980.1 & tle1 & Tle1 & K24V & 26 & 0 \\
\hline 93 & NP_251980.1 & tle1 & Tle1 & E27K & 26 & 0 \\
\hline 94 & NP_251980.1 & tle1 & Tle1 & L30S & 26 & 0 \\
\hline 95 & NP_251980.1 & tle1 & Tle1 & K31R & 26 & 0 \\
\hline 96 & NP_251980.1 & tle1 & Tle1 & Q33V & 26 & 0 \\
\hline 97 & NP_251980.1 & tle1 & Tle1 & D35E & 26 & 0 \\
\hline 98 & NP_251980.1 & tle1 & Tle1 & V38K & 26 & 0 \\
\hline 99 & NP_251980.1 & tle1 & Tle1 & F39Y & 26 & 0 \\
\hline 100 & NP_251980.1 & tle1 & Tle1 & S40N & 26 & 0 \\
\hline 101 & NP_251980.1 & tle1 & Tle1 & V54S & 26 & 0 \\
\hline 102 & NP_251980.1 & tle1 & Tle1 & G55S & 26 & 0 \\
\hline 103 & NP_251980.1 & tle1 & Tle1 & A57I & 26 & 0 \\
\hline 104 & NP_251980.1 & tle1 & Tle1 & T66S & 26 & 0 \\
\hline 105 & NP_251980.1 & tle1 & Tle1 & L67I & 26 & 0 \\
\hline 106 & NP_251980.1 & tle1 & Tle1 & F69Y & 26 & 0 \\
\hline 107 & NP_251980.1 & tle1 & Tle1 & K77R & 26 & 0 \\
\hline 108 & NP_251980.1 & tle1 & Tle1 & V83L & 26 & 0 \\
\hline 109 & NP_251980.1 & tle1 & Tle1 & S84P & 26 & 0 \\
\hline 110 & NP_251980.1 & tle1 & Tle1 & C87R & 26 & 0 \\
\hline 111 & NP_251980.1 & tle1 & Tle1 & I98V & 26 & 0 \\
\hline 112 & NP_251980.1 & tle1 & Tle1 & S100E & 26 & 0 \\
\hline 113 & NP_251980.1 & tle1 & Tle1 & D103V & 26 & 0 \\
\hline 114 & NP_251980.1 & tle1 & Tle1 & I104Q & 26 & 0 \\
\hline 115 & NP_251980.1 & tle1 & Tle1 & S106G & 26 & 0 \\
\hline 116 & NP_251980.1 & tle1 & Tle1 & R107L & 26 & 0 \\
\hline 117 & NP_251980.1 & tle1 & Tle1 & K125R & 26 & 0 \\
\hline 118 & NP_251980.1 & tle1 & Tle1 & M132L & 26 & 0 \\
\hline 119 & NP_251980.1 & tle1 & Tle1 & G136A & 26 & 0 \\
\hline 120 & NP_251980.1 & tle1 & Tle1 & E138A & 26 & 0 \\
\hline
\end{tabular}




\begin{tabular}{lllllrr}
\hline Rank & Accession & $\begin{array}{c}\text { Gene } \\
\text { name }\end{array}$ & Protein name & Mutation & $\begin{array}{r}\text { Association } \\
\text { score }\end{array}$ & p value \\
\hline 121 & NP_251980.1 & tle1 & Tle1 & I144V & 26 & 0 \\
122 & NP_251980.1 & tle1 & Tle1 & L148I & 26 & 0 \\
123 & NP_251980.1 & tle1 & Tle1 & Q150R & 26 & 0 \\
124 & NP_251980.1 & tle1 & Tle1 & A154V & 26 & 0 \\
125 & NP_251980.1 & tle1 & Tle1 & F156Q & 26 & 0 \\
126 & NP_251980.1 & tle1 & Tle1 & Y157G & 26 & 0 \\
127 & NP_251980.1 & tle1 & Tle1 & L160T & 26 & 0 \\
128 & NP_251980.1 & tle1 & Tle1 & 160 & 26 & 0 \\
& & & & insertion & & \\
129 & NP_251980.1 & tle1 & Tle1 & K161E & 26 & 0 \\
130 & NP_251980.1 & tle1 & Tle1 & R163P & 26 & 0 \\
131 & NP_251980.1 & tle1 & Tle1 & K165L & 26 & 0 \\
132 & NP_251980.1 & tle1 & Tle1 & N167D & 26 & 0 \\
133 & NP_251980.1 & tle1 & Tle1 & V169S & 26 & 0 \\
134 & NP_251980.1 & tle1 & Tle1 & G171T & 26 & 0 \\
\hline
\end{tabular}

\section{B.11. Dataset 3 random forest results}

Table 28: Genes and SNPs identified with RF from dataset 3, combined gene presence/absence and SNPs within core genes. Variants with importances greater than or equal to 0.002 are reported.

\begin{tabular}{|c|c|c|c|c|c|}
\hline Rank & Accession & Gene name & Protein name & Mutation & Importance \\
\hline 1 & NP_250764.1 & & PA2074 & & 0.262 \\
\hline 2 & NP_259454.1 & mucA & anti-sigma factor MucA & & 0.073 \\
\hline 3 & NP_250663.1 & $p q q F$ & $\begin{array}{l}\text { pyrroloquinoline quinone } \\
\text { biosynthesis protein } \mathrm{F}\end{array}$ & H3R & 0.051 \\
\hline 4 & NP_258888.1 & tonB2 & TonB2 & & 0.038 \\
\hline 5 & NP_253724.1 & & PA5037 & & 0.022 \\
\hline 6 & - & & $\begin{array}{l}\text { non-ribosomal peptide synthetase } \\
\text { (pseudogene) }\end{array}$ & & 0.019 \\
\hline 7 & NP_252532.1 & & PA3843 & & 0.01 \\
\hline 8 & - & & mutL (pseudogene) & & 0.01 \\
\hline 9 & NP_250663.1 & $p q q F$ & $\begin{array}{l}\text { pyrroloquinoline quinone } \\
\text { biosynthesis protein F }\end{array}$ & $\mathrm{H} 12 \mathrm{R}$ & 0.01 \\
\hline 10 & NP_259620.1 & & two-component response regulator & & 0.009 \\
\hline 11 & - & & $5 S$ ribosomal RNA & & 0.009 \\
\hline 12 & - & & $\begin{array}{l}\text { DNA-binding response regulator } \\
\text { (pseudogene) }\end{array}$ & & 0.008 \\
\hline 13 & - & & $\mathrm{ffs}$ & & 0.007 \\
\hline 14 & $\bar{N} P \_253777.1$ & $\operatorname{vgr} G 5$ & VgrG5 & F699L & 0.007 \\
\hline 15 & & & hypothetical protein & & 0.007 \\
\hline 16 & $\bar{N} P \_252760.1$ & & PA4071 & & 0.007 \\
\hline 17 & - & & hypothetical protein & & 0.006 \\
\hline 18 & - & & hypothetical protein & & 0.006 \\
\hline 19 & NP_251831.1 & $w b p M$ & $\begin{array}{l}\text { nucleotide sugar } \\
\text { epimerase/dehydratase } \mathrm{WbpM}\end{array}$ & V113P & 0.005 \\
\hline
\end{tabular}




\begin{tabular}{|c|c|c|c|c|c|}
\hline Rank & Accession & Gene name & Protein name & Mutation & Importance \\
\hline 20 & NP_250828.1 & $\operatorname{lig} D$ & $\begin{array}{l}\text { Multifunctional non-homologous } \\
\text { end joining protein LigD }\end{array}$ & S538G & 0.005 \\
\hline 21 & _ & & hypothetical protein & & 0.005 \\
\hline 22 & _ & & hypothetical protein (pseudogene) & & 0.005 \\
\hline 23 & NP_249457.1 & muсD & serine protease MucD precursor & V441I & 0.005 \\
\hline 24 & NP_253893.1 & $\arg E$ & acetylornithine deacetylase & R221Q & 0.005 \\
\hline 25 & NP_249711.1 & & $\begin{array}{l}\text { PA1020 probable acyl-CoA } \\
\text { dehydrogenase }\end{array}$ & S49T & 0.005 \\
\hline 26 & NP_249004.1 & & $\begin{array}{l}\text { L-cysteine transporter of ABC } \\
\text { system YecS }\end{array}$ & $\begin{array}{l}164 \\
\text { insertion }\end{array}$ & 0.004 \\
\hline 27 & & & hypothetical protein & & 0.004 \\
\hline 28 & NP_252030.1 & & PA3340 & H311R & 0.004 \\
\hline 29 & NP_252032.1 & & PA3342 & $\mathrm{A} 40 \mathrm{P}$ & 0.004 \\
\hline 30 & NP_251980.1 & tle1 & Tle1 & D168E & 0.004 \\
\hline 31 & NP_249340.1 & $\operatorname{trp} G$ & anthranilate synthase component II & $\mathrm{D} 111 \mathrm{~N}$ & 0.004 \\
\hline 32 & NP_249933.1 & $\operatorname{sprP}$ & SprP & E120D & 0.004 \\
\hline 33 & NP_250710.1 & $\operatorname{mex} \mathrm{Z}$ & MexZ & & 0.004 \\
\hline 34 & NP_259117.1 & $\operatorname{mex} B$ & $\begin{array}{l}\text { Resistance-Nodulation-Cell } \\
\text { Division (RND) multidrug efflux } \\
\text { transporter MexB }\end{array}$ & & 0.004 \\
\hline 35 & NP_253820.1 & & PA5133 & & 0.004 \\
\hline 36 & NP_250616.1 & & PA1926 & V660L & 0.004 \\
\hline 37 & NP_254125.1 & & $\begin{array}{l}\text { PA5438 probable transcriptional } \\
\text { regulator }\end{array}$ & $\begin{array}{l}272 \\
\text { deletion }\end{array}$ & 0.004 \\
\hline 38 & NP_250959.1 & & PA2269 & R204C & 0.004 \\
\hline 39 & NP_250980.1 & $g c d$ & glucose dehydrogenase & & 0.004 \\
\hline 40 & NP_254723.1 & recF & RecF protein & & 0.003 \\
\hline 41 & NP_249098.1 & $g s h B$ & glutathione synthetase & R266L & 0.003 \\
\hline 42 & NP_249438.1 & & $\begin{array}{l}\text { PA0747 probable aldehyde } \\
\text { dehydrogenase }\end{array}$ & V22I & 0.003 \\
\hline 43 & NP_249973.1 & & $\begin{array}{l}\text { PA1282 probable major facilitator } \\
\text { superfamily (MFS) transporter }\end{array}$ & T219A & 0.003 \\
\hline 44 & - & & hypothetical protein & & 0.003 \\
\hline 45 & NP_253977.1 & & PA5290 & M494T & 0.003 \\
\hline 46 & NP_250152.1 & $\operatorname{mot} D$ & MotD & D107G & 0.003 \\
\hline 47 & NP_249939.1 & aprF & $\begin{array}{l}\text { Alkaline protease secretion outer } \\
\text { membrane protein AprF precursor }\end{array}$ & T82A & 0.003 \\
\hline 48 & NP_252955.1 & tufA & elongation factor $\mathrm{Tu}$ & & 0.003 \\
\hline 49 & - & & & & 0.003 \\
\hline 50 & NP_253236.1 & pils & two-component sensor PilS & & 0.003 \\
\hline 51 & NP_251134.1 & $g l y A 2$ & serine hydroxymethyltransferase & & 0.003 \\
\hline 52 & - & & $\begin{array}{l}\text { formate dehydrogenase-N subunit } \\
\text { alpha (pseudogene) }\end{array}$ & & 0.003 \\
\hline 53 & YP_003933608.1 & & PA0457.1 & & 0.003 \\
\hline 54 & & & tRNA-Ile & & 0.003 \\
\hline 55 & NP_249960.1 & & $\begin{array}{l}\text { PA1269 probable transcriptional } \\
\text { regulator }\end{array}$ & V95A & 0.003 \\
\hline 56 & - & & $\begin{array}{l}\text { cystine } A B C \text { transporter } \\
\text { substrate-binding protein } \\
\text { (pseudogene) }\end{array}$ & & 0.003 \\
\hline
\end{tabular}


B. RESULTS

\begin{tabular}{|c|c|c|c|c|c|}
\hline Rank & Accession & Gene name & Protein name & Mutation & Importance \\
\hline 57 & NP_250088.1 & & $\begin{array}{l}\text { PA1397 probable two-component } \\
\text { response regulator }\end{array}$ & D45G & 0.003 \\
\hline 58 & & & protease (pseudogene) & & 0.003 \\
\hline 59 & NP_252899.1 & phzA1 & $\begin{array}{l}\text { probable phenazine biosynthesis } \\
\text { protein }\end{array}$ & & 0.003 \\
\hline 60 & NP_248956.1 & $\operatorname{dav} D$ & $\begin{array}{l}\text { glutaric semialdehyde } \\
\text { dehydrogenase }\end{array}$ & A338T & 0.003 \\
\hline 61 & & & & & 0.003 \\
\hline 62 & NP_250540.1 & & PA1849 & V38T & 0.003 \\
\hline 63 & NP_064724.1 & gyrB & DNA gyrase subunit B & S466F & 0.003 \\
\hline 64 & NP_250663.1 & $p q q F$ & $\begin{array}{l}\text { pyrroloquinoline quinone } \\
\text { biosynthesis protein } \mathrm{F}\end{array}$ & T206A & 0.002 \\
\hline 65 & NP_250871.1 & & 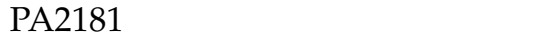 & & 0.002 \\
\hline 66 & NP_251359.1 & & PA2669 & Q149E & 0.002 \\
\hline 67 & NP_250244.1 & ccoO1 & $\begin{array}{l}\text { Cytochrome c oxidase, cbb3-type, } \\
\text { CcoO subunit }\end{array}$ & & 0.002 \\
\hline 68 & NP_249262.1 & & 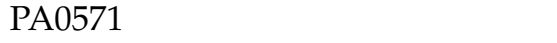 & $\mathrm{R} 42 \mathrm{H}$ & 0.002 \\
\hline 69 & NP_249110.1 & & PA0419 & & 0.002 \\
\hline 70 & NP_252360.1 & & PA3670 & & 0.002 \\
\hline 71 & NP_252831.1 & & PA4142 probable secretion protein & Q163R & 0.002 \\
\hline 72 & 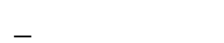 & & hypothetical protein & & 0.002 \\
\hline 73 & NP_249170.1 & & $\begin{array}{l}\text { PA0479 probable transcriptional } \\
\text { regulator }\end{array}$ & G83D & 0.002 \\
\hline 74 & NP_253393.1 & phuW & PhuW & E125Q & 0.002 \\
\hline 75 & NP_253525.1 & & PA4838 & A166T & 0.002 \\
\hline 76 & NP_252799.1 & $\operatorname{ampC}$ & beta-lactamase precursor & & 0.002 \\
\hline 77 & NP_250404.1 & $\operatorname{exs} A$ & transcriptional regulator ExsA & & 0.002 \\
\hline 78 & NP_252538.1 & & PA3849 & M315T & 0.002 \\
\hline 79 & NP_252405.1 & & PA3716 & S374R & 0.002 \\
\hline 80 & NP_249981.1 & & $\begin{array}{l}\text { PA1290 probable transcriptional } \\
\text { regulator }\end{array}$ & N175S & 0.002 \\
\hline 81 & NP_251450.1 & oprQ & OprQ & K56Q & 0.002 \\
\hline 82 & NP_253191.1 & opdD & $\begin{array}{l}\text { Glycine-glutamate dipeptide porin } \\
\text { OpdP }\end{array}$ & A19S & 0.002 \\
\hline 83 & - & & $\begin{array}{l}\text { pilus assembly protein FimV } \\
\text { (pseudogene) }\end{array}$ & & 0.002 \\
\hline 84 & NP_253350.1 & pagL & Lipid A 3-O-deacylase & & 0.002 \\
\hline 85 & NP_250177.1 & bapF & beta-peptidyl aminopeptidase & $\mathrm{I} 264 \mathrm{~V}$ & 0.002 \\
\hline 86 & NP_254219.1 & & PA5532 & $\begin{array}{l}252 \\
\text { insertion }\end{array}$ & 0.002 \\
\hline 87 & NP_251044.1 & & $\begin{array}{l}\text { PA2354 probable transcriptional } \\
\text { regulator }\end{array}$ & $\mathrm{T} 78 \mathrm{~N}$ & 0.002 \\
\hline 88 & NP_253462.1 & & PA4774 & X350E & 0.002 \\
\hline 89 & NP_251228.1 & & PA2538 & I143V & 0.002 \\
\hline 90 & - & & & & 0.002 \\
\hline 91 & NP_250018.1 & & PA1327 probable protease & & 0.002 \\
\hline 92 & NP_251858.1 & gyrA & DNA gyrase subunit A & T83I & 0.002 \\
\hline 93 & NP_249092.1 & & $\begin{array}{l}\text { noncatalytic dihydroorotase-like } \\
\text { protein }\end{array}$ & G130S & 0.002 \\
\hline
\end{tabular}




\begin{tabular}{|c|c|c|c|c|c|}
\hline Rank & Accession & Gene name & Protein name & Mutation & Importance \\
\hline 94 & NP_250515.1 & & PA1824 & A148V & 0.002 \\
\hline 95 & NP_252304.1 & & PA3614 & S446G & 0.002 \\
\hline 96 & NP_252801.1 & & $\begin{array}{l}\text { PA4112 probable sensor/response } \\
\text { regulator hybrid }\end{array}$ & A1110T & 0.002 \\
\hline 97 & NP_249826.1 & & PA1135 & G210D & 0.002 \\
\hline 98 & NP_252644.1 & & PA3955 & A191T & 0.002 \\
\hline 99 & & & $\mathrm{~N}$-acetyltransferase GCN5 & & 0.002 \\
\hline 100 & NP_250663.1 & $p q q F$ & $\begin{array}{l}\text { pyrroloquinoline quinone } \\
\text { biosynthesis protein F }\end{array}$ & K92R & 0.002 \\
\hline 101 & NP 250442.1 & & PA1751 1 1 & A2V & 0.002 \\
\hline 102 & NP_253231.1 & lepA & $\begin{array}{l}\text { Pseudomonas aeruginosa-derived } \\
\text { large extracellular protease, LepA }\end{array}$ & D769N & 0.002 \\
\hline 103 & NP_253340.1 & cupE4 & Pilin assembly chaperone CupE4 & R228Q & 0.002 \\
\hline 104 & NP_250144.1 & flhF & flagellar biosynthesis protein FlhF & $\mathrm{T} 84 \mathrm{~N}$ & 0.002 \\
\hline 105 & NP_251604.1 & & $\begin{array}{l}\text { PA2914 probable permease of } \mathrm{ABC} \\
\text { transporter }\end{array}$ & G218C & 0.002 \\
\hline 106 & NP_249416.1 & & $\begin{array}{l}\text { PA0725 hypothetical protein of } \\
\text { bacteriophage Pf1 }\end{array}$ & & 0.002 \\
\hline 107 & NP_252716.1 & & PA4027 & P293S & 0.002 \\
\hline 108 & NP_248817.1 & & PA0127 & Q125R & 0.002 \\
\hline 109 & NP_248827.1 & & $\begin{array}{l}\text { PA0137 probable permease of } \mathrm{ABC} \\
\text { transporter }\end{array}$ & S326T & 0.002 \\
\hline 110 & NP_251831.1 & $w b p M$ & $\begin{array}{l}\text { nucleotide sugar } \\
\text { epimerase/dehydratase WbpM }\end{array}$ & S51G & 0.002 \\
\hline 111 & NP_253996.1 & pauB4 & FAD-dependent oxidoreductase & T233A & 0.002 \\
\hline 112 & NP_251800.1 & & PA3110 & & 0.002 \\
\hline 113 & NP_249692.1 & phnA & anthranilate synthase component I & D91A & 0.002 \\
\hline
\end{tabular}

\section{B.12. Dataset 3 support vector machine results}

Table 29: Genes and SNPs identified with SVM from dataset 3, combined gene presence/absence and SNPs within core genes. Top 100 variants with absolute hyperplane coefficients greater than 0.015 are reported.

\begin{tabular}{llllll}
\hline Rank & Accession & Gene name & Protein name & $\begin{array}{c}\text { Mutation } \\
\text { Hyperplane } \\
\text { coefficient }\end{array}$ \\
\hline 1 & NP_249454.1 & mucA & $\begin{array}{l}\text { anti-sigma factor MucA } \\
\text { dioxygenase } \\
\text { dioxygenase }\end{array}$ & -0.0432 \\
3 & - & & $\begin{array}{l}\text { 2,3-dihydro-3-hydroxyanthranilate } \\
\text { isomerase }\end{array}$ & -0.0337 \\
4 & - & & TonB2 & 0.0320 \\
& & & PA1345 & L17P & -0.0315 \\
5 & NP_248888.1 & \multirow{2}{*}{ tonB2 } & Resistance-Nodulation-Cell & 0.0284 \\
6 & NP_250036.1 & & Division (RND) multidrug efflux & & -0.0281 \\
7 & NP_249116.1 & mexA & membrane fusion protein MexA & & \\
& & & precursor & & \\
& & & L-cysteine transporter of ABC & 164 & 0.0279 \\
8 & NPstem YecS & insertion & \\
\hline
\end{tabular}


B. RESULTS

\begin{tabular}{|c|c|c|c|c|c|}
\hline Rank & Accession & Gene name & Protein name & Mutation & $\begin{array}{l}\text { Hyperplane } \\
\text { coefficient }\end{array}$ \\
\hline 9 & & oprB & Glucose-selective porin OprB & & 0.0277 \\
\hline 10 & NP_250674.1 & exaC & $\begin{array}{l}\text { NAD+ dependent aldehyde } \\
\text { dehydrogenase ExaC }\end{array}$ & & -0.0262 \\
\hline 11 & _ & & addiction module antitoxin RelB & & 0.0261 \\
\hline 12 & . & & hypothetical protein & & 0.0255 \\
\hline 13 & NP_253000.1 & $p c t B$ & chemotactic transducer PctB & & 0.0255 \\
\hline 14 & NP_253462.1 & & PA4774 & $\begin{array}{l}308 \\
\text { deletion }\end{array}$ & -0.0249 \\
\hline 15 & NP_253462.1 & & PA4774 & X350E & -0.0249 \\
\hline 16 & NP_249622.1 & pirA & ferric enterobactin receptor PirA & A370T & -0.0244 \\
\hline 17 & - & & $\begin{array}{l}\text { integrating conjugative element } \\
\text { protein }\end{array}$ & & 0.0239 \\
\hline 18 & NP_251876.1 & oprB & $\begin{array}{l}\text { Glucose/carbohydrate outer } \\
\text { membrane porin OprB precursor }\end{array}$ & & -0.0237 \\
\hline 19 & NP_250710.1 & $\operatorname{mex} \mathrm{Z}$ & $\operatorname{MexZ}$ & & -0.0235 \\
\hline 20 & - $\quad$ n & & hypothetical protein & & 0.0227 \\
\hline 21 & NP_251835.1 & $w b p L$ & glycosyltransferase WbpL & & 0.0225 \\
\hline 22 & NP_252321.1 & & PA3631 & $\begin{array}{l}377 \\
\text { deletion }\end{array}$ & -0.0224 \\
\hline 23 & NP_252321.1 & & PA3631 & X409L & -0.0224 \\
\hline 24 & NP_248785.1 & & PA0095 & & 0.0222 \\
\hline 25 & NP_254125.1 & & $\begin{array}{l}\text { PA5438 probable transcriptional } \\
\text { regulator }\end{array}$ & $\begin{array}{l}272 \\
\text { deletion }\end{array}$ & 0.0219 \\
\hline 26 & - & & hypothetical protein & & -0.0216 \\
\hline 27 & _ & & hypothetical protein & & 0.0216 \\
\hline 28 & NP_250663.1 & $p q q F$ & $\begin{array}{l}\text { pyrroloquinoline quinone } \\
\text { biosynthesis protein } \mathrm{F}\end{array}$ & H3R & 0.0216 \\
\hline 29 & _- & & transcriptional regulator & & 0.0215 \\
\hline 30 & _ & & hypothetical protein & & 0.0215 \\
\hline 31 & - & & hypothetical protein & & -0.0215 \\
\hline 32 & NP_250663.1 & $p q q F$ & $\begin{array}{l}\text { pyrroloquinoline quinone } \\
\text { biosynthesis protein } \mathrm{F}\end{array}$ & $\mathrm{H} 12 \mathrm{R}$ & 0.0215 \\
\hline 33 & - & & type IV B pilus protein & & 0.0214 \\
\hline 34 & - & & acetyl-CoA acetyltransferase & & 0.0214 \\
\hline 35 & - & & hypothetical protein & & 0.0214 \\
\hline 36 & - & & hypothetical protein & & 0.0213 \\
\hline 37 & - & & $\begin{array}{l}\text { group II intron reverse } \\
\text { transcriptase/maturase }\end{array}$ & & 0.0211 \\
\hline 38 & & & protease (pseudogene) & & 0.0211 \\
\hline 39 & NP_253373.1 & & PA4684 & $\begin{array}{l}407 \\
\text { deletion }\end{array}$ & -0.0211 \\
\hline 40 & NP_253373.1 & & PA4684 & X433L & -0.0211 \\
\hline 41 & _ & & hypothetical protein & & 0.0210 \\
\hline 42 & _ & & endodeoxyribonuclease RusA & & 0.0208 \\
\hline 43 & _ & & hypothetical protein & & -0.0203 \\
\hline 44 & _- & & hypothetical protein & & 0.0202 \\
\hline 45 & _ & & type IV pilus protein PilV & & 0.0202 \\
\hline 46 & _- & & hypothetical protein & & 0.0201 \\
\hline
\end{tabular}


B. RESULTS

\begin{tabular}{|c|c|c|c|c|c|}
\hline Rank & Accession & Gene name & Protein name & Mutation & $\begin{array}{l}\text { Hyperplane } \\
\text { coefficient }\end{array}$ \\
\hline 47 & - & & toxin transporter & & 0.0201 \\
\hline 48 & - & & hypothetical protein & & 0.0200 \\
\hline 49 & _ & & hypothetical protein & & 0.0200 \\
\hline 50 & NP_254102.1 & glyA1 & serine hydroxymethyltransferase & & -0.0199 \\
\hline 51 & - & & hypothetical protein & & 0.0197 \\
\hline 52 & & & hypothetical protein (pseudogene) & & -0.0195 \\
\hline 53 & NP_251029.1 & & $\begin{array}{l}\text { PA2339 probable } \\
\text { binding-protein-dependent } \\
\text { maltose/mannitol transport protein }\end{array}$ & & -0.0195 \\
\hline 54 & NP_253781.1 & & $\begin{array}{l}\text { PA5094 probable ATP-binding } \\
\text { component of ABC transporter }\end{array}$ & $\begin{array}{l}269 \\
\text { deletion }\end{array}$ & -0.0194 \\
\hline 55 & NP_253781.1 & & $\begin{array}{l}\text { PA5094 probable ATP-binding } \\
\text { component of ABC transporter }\end{array}$ & X277Q & -0.0194 \\
\hline 56 & NP_251142 & & PA2452 & & -0.0194 \\
\hline 57 & - & & porin (pseudogene) & & -0.0194 \\
\hline 58 & _ & & twitching motility protein PilT & & -0.0192 \\
\hline 59 & _ & & hypothetical protein & & 0.0189 \\
\hline 60 & _ & & hypothetical protein & & -0.0188 \\
\hline 61 & NP_249374.1 & $h x_{c} Y$ & $\mathrm{HxcY}$ & & -0.0188 \\
\hline 62 & - & & hypothetical protein & & -0.0187 \\
\hline 63 & _ & & hypothetical protein & & -0.0187 \\
\hline 64 & - & & $\begin{array}{l}\text { type VI secretion protein ImpA } \\
\text { (pseudogene) }\end{array}$ & & -0.0186 \\
\hline 65 & - & & hypothetical protein & & -0.0186 \\
\hline 66 & - & & $\begin{array}{l}\text { bifunctional prephenate } \\
\text { dehydrogenase/3- } \\
\text { phosphoshikimate } \\
\text { 1-carboxyvinyltransferase }\end{array}$ & & -0.0183 \\
\hline 67 & - & & hypothetical protein & & -0.0183 \\
\hline 68 & NP_251023.1 & & PA2333 probable sulfatase & & -0.0183 \\
\hline 69 & NP_251025.1 & & $\begin{array}{l}\text { PA2335 probable TonB-dependent } \\
\text { receptor }\end{array}$ & & -0.0182 \\
\hline 70 & NP_250922.1 & $p s l B$ & PslB & & -0.0182 \\
\hline 71 & - & & hypothetical protein & & -0.0182 \\
\hline 72 & - & & major exported protein & & -0.0181 \\
\hline 73 & NP_250881.1 & exoY & adenylate cyclase ExoY & & -0.0181 \\
\hline 74 & & & acetyltransferase & & 0.0179 \\
\hline 75 & NP_251114.1 & $p v d L$ & PvdL & V3938I & -0.0179 \\
\hline 76 & _- & & hypothetical protein & & 0.0178 \\
\hline 77 & - & & hypothetical protein & & -0.0178 \\
\hline 78 & & & hypothetical protein & & 0.0178 \\
\hline 79 & NP_249552.1 & $\operatorname{rbdA}$ & $\mathrm{RbDA}$ & & -0.0178 \\
\hline 80 & & & hypothetical protein & & -0.0178 \\
\hline 81 & NP_251148.1 & & PA2458 & & -0.0177 \\
\hline 82 & _- & & phosphodiesterase & & -0.0177 \\
\hline 83 & _- & & cobalt transporter & & -0.0177 \\
\hline 84 & _ & & $23 S$ ribosomal RNA & & -0.0174 \\
\hline 85 & _ & & integrase & & 0.0174 \\
\hline
\end{tabular}




\begin{tabular}{|c|c|c|c|c|c|}
\hline Rank & Accession & Gene name & Protein name & Mutation & $\begin{array}{l}\text { Hyperplane } \\
\text { coefficient }\end{array}$ \\
\hline 86 & _- & & hypothetical protein & & 0.0173 \\
\hline 87 & _ & & hypothetical protein & & 0.0173 \\
\hline 88 & _ & & membrane protein & & -0.0173 \\
\hline 89 & _ & & hypothetical protein & & -0.0173 \\
\hline 90 & _ & & hypothetical protein & & -0.0173 \\
\hline 91 & _ & & DNA methyltransferase & & 0.0173 \\
\hline 92 & _ & & transporter & & -0.0173 \\
\hline 93 & _ & & type IV B pilus protein & & -0.0172 \\
\hline 94 & _ & & lipoprotein & & 0.0172 \\
\hline 95 & - & & $\begin{array}{l}\text { RNA polymerase subunit sigma } \\
\text { (pseudogene) }\end{array}$ & & 0.0172 \\
\hline 96 & - & & acetyl-CoA acetyltransferase & & -0.0171 \\
\hline 97 & _ & & paraquat-inducible protein $\mathrm{A}$ & & 0.0171 \\
\hline 98 & _- & & hypothetical protein & & -0.0171 \\
\hline 99 & NP_064723.1 & recF & RecF protein & & -0.0170 \\
\hline 100 & - & & hypothetical protein & & 0.0170 \\
\hline 101 & _ & & hypothetical protein & & 0.0170 \\
\hline
\end{tabular}

\section{B.13. Dataset 4 PLINK results}

Table 30: Genes and SNPs identified with PLINK from dataset 4, reduced dataset using features identified from random forest in datasets 1 and 2 . Variants with $p$ values less than $1 \times 10^{-8}$ are reported.

\begin{tabular}{|c|c|c|c|c|c|c|}
\hline Rank & Accession & $\begin{array}{l}\text { Gene } \\
\text { name }\end{array}$ & Protein name & Mutation & p value & $\begin{array}{l}\text { Odds } \\
\text { ratio }\end{array}$ \\
\hline 1 & NP 248888.1 & tonB2 & TonB2 & & $2.677 \times 10^{-60}$ & 24.43 \\
\hline 2 & NP_253781.1 & & $\begin{array}{l}\text { PA5094 probable } \\
\text { ATP-binding component } \\
\text { of ABC transporter }\end{array}$ & X277Q & $1.590 \times 10^{-49}$ & 0.09 \\
\hline 3 & NP_253957.1 & & PA5270 & S5A & $3.663 \times 10^{-49}$ & 9.21 \\
\hline 4 & - & & hypothetical protein & & $1.793 \times 10^{-45}$ & 10.55 \\
\hline 5 & NP_253462.1 & & PA4774 & X350E & $8.968 \times 10^{-44}$ & 0.10 \\
\hline 6 & - & & $\begin{array}{l}\text { DNA-binding response } \\
\text { regulator (pseudogene) }\end{array}$ & & $3.371 \times 10^{-39}$ & 2077 \\
\hline 7 & NP_253159.1 & & PA4469 & & $3.106 \times 10^{-37}$ & NA \\
\hline 8 & NP_249374.1 & $h x c Y$ & $\mathrm{HxcY}$ & & $2.769 \times 10^{-36}$ & 30.01 \\
\hline 9 & NP_249381.1 & $p d t A$ & $\begin{array}{l}\text { phosphate depletion } \\
\text { regulated TPS partner A, } \\
\text { PdtA }\end{array}$ & & $1.081 \times 10^{-34}$ & 21.71 \\
\hline 10 & - & & hypothetical protein & & $1.119 \times 10^{-33}$ & 7.16 \\
\hline 11 & & & hypothetical protein & & $6.548 \times 10^{-33}$ & 169.20 \\
\hline 12 & NP_250663.1 & $p q q F$ & $\begin{array}{l}\text { pyrroloquinoline quinone } \\
\text { biosynthesis protein } \mathrm{F}\end{array}$ & $\mathrm{H} 12 \mathrm{R}$ & $3.887 \times 10^{-32}$ & 0.18 \\
\hline 13 & NP_064723.1 & $\mathrm{recF}$ & RecF protein & & $1.478 \times 10^{-31}$ & 39.57 \\
\hline 14 & NP_249865.1 & napA & $\begin{array}{l}\text { periplasmic nitrate } \\
\text { reductase protein NapA }\end{array}$ & & $4.727 \times 10^{-31}$ & 24.37 \\
\hline
\end{tabular}




\begin{tabular}{|c|c|c|c|c|c|c|}
\hline Rank & Accession & $\begin{array}{l}\text { Gene } \\
\text { name }\end{array}$ & Protein name & Mutation & p value & $\begin{array}{l}\text { Odds } \\
\text { ratio }\end{array}$ \\
\hline 15 & - & & pseudogene & & $5.190 \times 10^{-30}$ & 16.46 \\
\hline 16 & NP_249454.1 & muсA & anti-sigma factor MucA & & $8.940 \times 10^{-30}$ & 25.28 \\
\hline 17 & NP_250018.1 & & PA1327 probable protease & & $3.380 \times 10^{-29}$ & 85.36 \\
\hline 18 & NP_250663.1 & $p q q F$ & $\begin{array}{l}\text { pyrroloquinoline quinone } \\
\text { biosynthesis protein } \mathrm{F}\end{array}$ & H3R & $2.338 \times 10^{-27}$ & 0.21 \\
\hline 19 & NP_249117.1 & $\operatorname{mex} B$ & $\begin{array}{l}\text { Resistance-Nodulation- } \\
\text { Cell Division (RND) } \\
\text { multidrug efflux } \\
\text { transporter MexB }\end{array}$ & & $1.165 \times 10^{-26}$ & 18.91 \\
\hline 20 & NP_253474.1 & & $\begin{array}{l}\text { PA4786 probable } \\
\text { short-chain } \\
\text { dehydrogenase }\end{array}$ & & $4.820 \times 10^{-26}$ & 27.32 \\
\hline 21 & NP_253664.1 & aruI & $\begin{array}{l}\text { 2-ketoarginine } \\
\text { decarboxylase, AruI }\end{array}$ & & $7.288 \times 10^{-26}$ & 27.23 \\
\hline 22 & - & & $\begin{array}{l}\text { DNA mismatch repair } \\
\text { protein MutL } \\
\text { (pseudogene) }\end{array}$ & & $1.131 \times 10^{-25}$ & 11.29 \\
\hline 23 & NP_249234.1 & & PA0543 & & $1.178 \times 10^{-24}$ & 1414 \\
\hline 24 & NP_249929.1 & & $\begin{array}{l}\text { PA1238 probable outer } \\
\text { membrane component of } \\
\text { multidrug efflux pump }\end{array}$ & & $3.144 \times 10^{-24}$ & 6.14 \\
\hline 25 & NP_250447.1 & cys $H$ & $\begin{array}{l}3^{\prime} \text {-phosphoadenosine }-5^{\prime} \text { - } \\
\text { phosphosulfate } \\
\text { reductase }\end{array}$ & L2P & $5.950 \times 10^{-23}$ & 12.11 \\
\hline 26 & NP_252760.1 & & PA4071 & & $8.727 \times 10^{-23}$ & 11.69 \\
\hline 27 & - & & hypothetical protein & & $6.694 \times 10^{-22}$ & 11.20 \\
\hline 28 & NP_253053.1 & $i c i A$ & $\begin{array}{l}\text { inhibitor of chromosome } \\
\text { initiation IciA }\end{array}$ & M1L & $2.644 \times 10^{-21}$ & 11.68 \\
\hline 29 & NP_253724.1 & & PA5037 & & $3.797 \times 10^{-21}$ & 16.65 \\
\hline 30 & NP_249976.1 & & $\begin{array}{l}\text { PA1285 probable } \\
\text { transcriptional regulator }\end{array}$ & D3V & $4.551 \times 10^{-21}$ & 18.45 \\
\hline 31 & NP_253108.1 & ftsI & $\begin{array}{l}\text { penicillin-binding protein } \\
3\end{array}$ & R504C & $1.290 \times 10^{-20}$ & 70.55 \\
\hline 32 & - & & $\begin{array}{l}\text { fused response } \\
\text { regulator/phosphatase } \\
\text { (pseudogene) }\end{array}$ & & $8.894 \times 10^{-20}$ & 58.28 \\
\hline 33 & - & & transporter (pseudogene) & & $1.339 \times 10^{-19}$ & 1082 \\
\hline 34 & - & & $\begin{array}{l}\text { 3-oxoacyl-ACP reductase } \\
\text { (pseudogene) }\end{array}$ & & $1.426 \times 10^{-19}$ & 7.29 \\
\hline 35 & NP_249140.1 & & PA0449 & W38R & $5.887 \times 10^{-19}$ & 26.55 \\
\hline 36 & NP_249140.1 & & PA0449 & G39S & $5.887 \times 10^{-19}$ & 26.55 \\
\hline 37 & NP_250674.1 & $\operatorname{exaC}$ & $\begin{array}{l}\text { NAD+ dependent } \\
\text { aldehyde dehydrogenase } \\
\text { ExaC }\end{array}$ & & $2.493 \times 10^{-18}$ & 4.02 \\
\hline 38 & NP_251593.1 & $c o b J$ & $\begin{array}{l}\text { precorrin-3 methylase } \\
\text { CobJ }\end{array}$ & T4A & $3.509 \times 10^{-18}$ & 4.60 \\
\hline 39 & NP_254125.1 & & $\begin{array}{l}\text { PA5438 probable } \\
\text { transcriptional regulator }\end{array}$ & $\begin{array}{l}272 \\
\text { deletion }\end{array}$ & $6.325 \times 10^{-17}$ & 31.10 \\
\hline
\end{tabular}




\begin{tabular}{|c|c|c|c|c|c|c|}
\hline Rank & Accession & $\begin{array}{l}\text { Gene } \\
\text { name }\end{array}$ & Protein name & Mutation & $\mathrm{p}$ value & $\begin{array}{l}\text { Odds } \\
\text { ratio }\end{array}$ \\
\hline 40 & NP_253015.1 & & PA4325 & K28E & $7.394 \times 10^{-17}$ & NA \\
\hline 41 & NP_064724.1 & gyrB & DNA gyrase subunit B & S466F & $2.944 \times 10^{-16}$ & 480.90 \\
\hline 42 & NP_253777.1 & vgrG5 & VgrG5 & $\mathrm{A} 242 \mathrm{~T}$ & $5.622 \times 10^{-16}$ & 6.06 \\
\hline 43 & NP_248891.1 & & PA0200 & E64D & $6.834 \times 10^{-16}$ & 4.01 \\
\hline 44 & NP_251237.1 & & $\begin{array}{l}\text { PA2547 probable } \\
\text { transcriptional regulator }\end{array}$ & E124D & $1.049 \times 10^{-16}$ & 0.18 \\
\hline 45 & NP_252799.1 & $\operatorname{ampC}$ & beta-lactamase precursor & V239A & $2.917 \times 10^{-15}$ & NA \\
\hline 46 & - & & $\begin{array}{l}\text { cystine } \mathrm{ABC} \text { transporter } \\
\text { substrate-binding protein } \\
\text { (pseudogene) }\end{array}$ & & $8.836 \times 10^{-15}$ & NA \\
\hline 47 & NP_248841.1 & & $\begin{array}{l}\text { PA0151probable } \\
\text { TonB-dependent receptor }\end{array}$ & E597Q & $1.416 \times 10^{-14}$ & 0.12 \\
\hline 48 & NP_251742.1 & & PA3052 & $\mathrm{H} 18 \mathrm{R}$ & $1.924 \times 10^{-14}$ & 8.59 \\
\hline 49 & NP_253604.1 & nadD2 & $\begin{array}{l}\text { nicotinate } \\
\text { mononucleotide } \\
\text { adenylyltransferase } \\
\text { NadD2 }\end{array}$ & S7Y & $2.039 \times 10^{-14}$ & 4.35 \\
\hline 50 & - & & transporter & & $2.764 \times 10^{-14}$ & 0.28 \\
\hline 51 & NP_248708.1 & fmt & $\begin{array}{l}\text { methionyl-tRNA } \\
\text { formyltransferase }\end{array}$ & I196V & $3.410 \times 10^{-14}$ & 0.22 \\
\hline 52 & - & & hypothetical protein & & $1.255 \times 10^{-13}$ & 0.28 \\
\hline 53 & NP_249116.1 & $\operatorname{mex} A$ & $\begin{array}{l}\text { Resistance-Nodulation- } \\
\text { Cell Division (RND) } \\
\text { multidrug efflux } \\
\text { membrane fusion protein } \\
\text { MexA precursor }\end{array}$ & & $2.070 \times 10^{-13}$ & 12.25 \\
\hline 54 & NP_253847.1 & & drug efflux transporter & & $2.767 \times 10^{-13}$ & 34.00 \\
\hline 55 & - & & hypothetical protein & & $3.430 \times 10^{-13}$ & 0.24 \\
\hline 56 & NP_253820.1 & & PA5133 & & $3.921 \times 10^{-13}$ & 4.74 \\
\hline 57 & NP_253669 & & $\begin{array}{l}\text { PA4982 probable } \\
\text { two-component sensor }\end{array}$ & N904S & $4.612 \times 10^{-13}$ & 20.17 \\
\hline 58 & - & & $\begin{array}{l}\text { aldehyde dehydrogenase } \\
\text { (pseudogene) }\end{array}$ & & $1.102 \times 10^{-12}$ & 12.00 \\
\hline 59 & NP_251831.1 & $w b p M$ & $\begin{array}{l}\text { nucleotide sugar } \\
\text { epimerase/dehydratase } \\
\text { WbpM }\end{array}$ & V113P & $1.296 \times 10^{-12}$ & 0.17 \\
\hline 60 & NP_249842.1 & $i m m 2$ & $\begin{array}{l}\text { pyocin S2 immunity } \\
\text { protein }\end{array}$ & & $1.308 \times 10^{-12}$ & 0.26 \\
\hline 61 & NP_254006.1 & $\mathrm{radC}$ & DNA repair protein RadC & G73A & $1.345 \times 10^{-12}$ & 0.06 \\
\hline 62 & NP_254006.1 & $\mathrm{radC}$ & DNA repair protein $\mathrm{RadC}$ & D212G & $1.345 \times 10^{-12}$ & 0.06 \\
\hline 63 & NP_254006.1 & $\mathrm{radC}$ & DNA repair protein RadC & G213N & $1.345 \times 10^{-12}$ & 0.06 \\
\hline 64 & NP_251813.1 & & PA3123 & S37T & $1.772 \times 10^{-12}$ & 0.28 \\
\hline 65 & NP_251232.1 & & PA2542 & $\begin{array}{l}1130 \\
\text { insertion }\end{array}$ & $3.018 \times 10^{-12}$ & NA \\
\hline 66 & _- & & hypothetical protein & & $3.890 \times 10^{-12}$ & 4.42 \\
\hline
\end{tabular}




\begin{tabular}{|c|c|c|c|c|c|c|}
\hline Rank & Accession & $\begin{array}{l}\text { Gene } \\
\text { name }\end{array}$ & Protein name & Mutation & p value & $\begin{array}{l}\text { Odds } \\
\text { ratio }\end{array}$ \\
\hline 67 & NP_249718.1 & $a m a B$ & $\begin{array}{l}\text { delta1-Piperideine-6- } \\
\text { carboxylate } \\
\text { dehydrogenase }\end{array}$ & A253D & $5.582 \times 10^{-12}$ & 4.98 \\
\hline 68 & NP_252709.1 & $m p l$ & $\begin{array}{l}\text { UDP-N- } \\
\text { acetylmuramate:L-alanyl- } \\
\text { gamma-D-glutamyl- } \\
\text { meso-diaminopimelate } \\
\text { ligase }\end{array}$ & & $5.886 \times 10^{-12}$ & 17.06 \\
\hline 69 & NP_249004.1 & & $\begin{array}{l}\text { L-cysteine transporter of } \\
\text { ABC system YecS }\end{array}$ & $\begin{array}{l}164 \\
\text { insertion }\end{array}$ & $7.611 \times 10^{-12}$ & NA \\
\hline 70 & NP_249415.1 & & $\begin{array}{l}\text { PA0724 probable coat } \\
\text { protein A of } \\
\text { bacteriophage Pf1 }\end{array}$ & & $8.949 \times 10^{-12}$ & 0.32 \\
\hline 71 & NP_248733.1 & & PA0043 & W4R & $1.034 \times 10^{-11}$ & 4.66 \\
\hline 72 & - & & hypothetical protein & & $1.914 \times 10^{-11}$ & 0.10 \\
\hline 73 & $\overline{N P} 250922.1$ & $p s l B$ & PslB & & $2.495 \times 10^{-11}$ & 6.16 \\
\hline 74 & NP_249491.1 & & PA0800 & H78R & $2.584 \times 10^{-11}$ & 10.95 \\
\hline 75 & - & & $\begin{array}{l}\text { N-acetyltransferase } \\
\text { GCN5 }\end{array}$ & & $2.709 \times 10^{-11}$ & 0.36 \\
\hline 76 & - & & hypothetical protein & & $2.842 \times 10^{-11}$ & 9.74 \\
\hline 77 & NP_251089.1 & $p v d D$ & pyoverdine synthetase D & & $3.156 \times 10^{-11}$ & 2.59 \\
\hline 78 & NP_253485.1 & & $\begin{array}{l}\text { PA4797 probable } \\
\text { transposase }\end{array}$ & & $5.499 \times 10^{-11}$ & 0.28 \\
\hline 79 & NP_251376.1 & pfeR & $\begin{array}{l}\text { two-component response } \\
\text { regulator PfeR }\end{array}$ & V281A & $1.101 \times 10^{-10}$ & 0.16 \\
\hline 80 & NP_249109.1 & & PA0418 & T455R & $1.769 \times 10^{-10}$ & 2.83 \\
\hline 81 & NP_250108.1 & & $\begin{array}{l}\text { PA1417 probable } \\
\text { decarboxylase }\end{array}$ & T262A & $1.903 \times 10^{-10}$ & 3.06 \\
\hline 82 & NP_252773.1 & сupB3 & usher CupB3 & & $2.964 \times 10^{-10}$ & 8.44 \\
\hline 83 & NP_250640.1 & $r b s K$ & ribokinase & L129M & $3.806 \times 10^{-10}$ & 0.07 \\
\hline 84 & NP_249286.1 & $\operatorname{lptD}$ & $\begin{array}{l}\text { LPS-assembly protein } \\
\text { LptD }\end{array}$ & N734T & $3.890 \times 10^{-10}$ & 0.31 \\
\hline 85 & NP_253663.1 & $\operatorname{aruH}$ & $\begin{array}{l}\text { Arginine:Pyruvate } \\
\text { Transaminas, AruH }\end{array}$ & R84Q & $4.820 \times 10^{-10}$ & 0.21 \\
\hline 86 & NP_251980.1 & tle1 & Tle1 & N131S & $5.027 \times 10^{-10}$ & 11.03 \\
\hline 87 & - & & hypothetical protein & & $6.531 \times 10^{-10}$ & 2.98 \\
\hline 88 & - & & $\begin{array}{l}\text { UDP-N- } \\
\text { acetylmuramate:L-alanyl- } \\
\text { gamma-D-glutamyl- } \\
\text { meso-diaminopimelate } \\
\text { ligase (pseudogene) }\end{array}$ & & $6.735 \times 10^{-10}$ & 27.74 \\
\hline 89 & - & & hypothetical protein & & $8.905 \times 10^{-10}$ & 2.89 \\
\hline 90 & NP_248921.1 & $p c a B$ & $\begin{array}{l}\text { 3-carboxy-cis,cis- } \\
\text { muconate } \\
\text { cycloisomerase }\end{array}$ & A298T & $1.002 \times 10^{-9}$ & 0.09 \\
\hline 91 & NP_251114.1 & $p v d L$ & PvdL & V3938I & $1.077 \times 10^{-9}$ & 3.15 \\
\hline 92 & - & & hypothetical protein & & $1.098 \times 10^{-9}$ & 0.36 \\
\hline
\end{tabular}




\begin{tabular}{|c|c|c|c|c|c|c|}
\hline Rank & Accession & $\begin{array}{l}\text { Gene } \\
\text { name }\end{array}$ & Protein name & Mutation & $\mathrm{p}$ value & $\begin{array}{l}\text { Odds } \\
\text { ratio }\end{array}$ \\
\hline 93 & NP_253337.1 & cupE1 & Pilin subunit CupE1 & & $1.302 \times 10^{-9}$ & NA \\
\hline 94 & NP_249019.1 & $a a a A$ & $\begin{array}{l}\text { arginine-specific } \\
\text { autotransporter of } \\
\text { Pseudomonas aeruginosa, } \\
\text { AaaA }\end{array}$ & H516R & $1.363 \times 10^{-9}$ & 0.36 \\
\hline 95 & NP_251146 & & PA2456 & & $1.464 \times 10^{-9}$ & 0.27 \\
\hline 96 & NP_250319.1 & & $\begin{array}{l}\text { PA1628 probable } \\
\text { 3-hydroxyacyl-CoA } \\
\text { dehydrogenase }\end{array}$ & R48Q & $1.534 \times 10^{-9}$ & 0.15 \\
\hline 97 & NP_251184.1 & $\operatorname{mex} F$ & $\begin{array}{l}\text { Resistance-Nodulation- } \\
\text { Cell Division (RND) } \\
\text { multidrug efflux } \\
\text { transporter MexF }\end{array}$ & & $1.720 \times 10^{-9}$ & 7.39 \\
\hline 98 & NP_250030.1 & aatP & AatP & & $2.013 \times 10^{-9}$ & NA \\
\hline 99 & NP_251435.1 & & $\begin{array}{l}\text { PA2745 probable } \\
\text { hydrolase }\end{array}$ & L88G & $2.641 \times 10^{-9}$ & 10.29 \\
\hline 100 & NP_250246.1 & $\operatorname{ccoP} 2$ & $\begin{array}{l}\text { Cytochrome c oxidase, } \\
\text { cbb3-type, CcoP subunit }\end{array}$ & & $3.364 \times 10^{-9}$ & 10.46 \\
\hline 101 & NP_251439.1 & end $A$ & $\begin{array}{l}\text { DNA-specific } \\
\text { endonuclease I }\end{array}$ & D151I & $5.611 \times 10^{-9}$ & 21.44 \\
\hline 102 & NP_250442.1 & & PA1751 & $\begin{array}{l}1 \\
\text { insertion }\end{array}$ & $6.738 \times 10^{-9}$ & 2.82 \\
\hline 103 & NP_252454.1 & & PA3765 & S62R & $6.761 \times 10^{-9}$ & 0.27 \\
\hline 104 & NP_250687.1 & & $\begin{array}{l}\text { PA1997 probable } \\
\text { AMP-binding enzyme }\end{array}$ & A328T & $9.248 \times 10^{-9}$ & 22.50 \\
\hline
\end{tabular}

\section{B.14. Dataset 4 treeWAS results}

Table 31: Genes and SNPs identified with treeWAS from dataset 4, reduced dataset using features identified from random forest in datasets 1 and 2. Variants are ranked based on absolute simultaneous association score.

\begin{tabular}{|c|c|c|c|c|c|c|}
\hline Rank & Accession & $\begin{array}{l}\text { Gene } \\
\text { name }\end{array}$ & Protein name & Mutation & $\begin{array}{c}\text { Association } \\
\text { score }\end{array}$ & p value \\
\hline 1 & NP_250663.1 & $p q q F$ & $\begin{array}{l}\text { pyrroloquinoline quinone } \\
\text { biosynthesis protein } \mathrm{F}\end{array}$ & H3R & 50.5 & 0 \\
\hline 2 & NP_250018.1 & & PA1327 probable protease & & -50 & 0 \\
\hline 3 & NP_250663.1 & $p q q F$ & $\begin{array}{l}\text { pyrroloquinoline quinone } \\
\text { biosynthesis protein } \mathrm{F}\end{array}$ & $\mathrm{H} 12 \mathrm{R}$ & 49.5 & 0 \\
\hline 4 & NP_249374.1 & $h x_{c} Y$ & $\mathrm{HxcY}$ & & -49 & 0 \\
\hline 5 & NP_249865.1 & napA & $\begin{array}{l}\text { periplasmic nitrate } \\
\text { reductase protein NapA }\end{array}$ & & -46 & 0 \\
\hline 6 & NP_253053.1 & $i c i A$ & $\begin{array}{l}\text { inhibitor of chromosome } \\
\text { initiation IciA }\end{array}$ & M1L & 44 & 0 \\
\hline 7 & NP_248888.1 & tonB2 & TonB2 & & -44 & 0 \\
\hline 8 & NP_253957.1 & & PA5270 & S5A & 43 & 0 \\
\hline 9 & NP_064723.1 & $\operatorname{recF}$ & RecF protein & & -42 & 0 \\
\hline
\end{tabular}




\begin{tabular}{|c|c|c|c|c|c|c|}
\hline Rank & Accession & $\begin{array}{l}\text { Gene } \\
\text { name }\end{array}$ & Protein name & Mutation & $\begin{array}{c}\text { Association } \\
\text { score }\end{array}$ & p value \\
\hline 10 & NP_250442.1 & & PA1751 & $\begin{array}{l}1 \\
\text { insertion }\end{array}$ & -41 & 0 \\
\hline 11 & & & hypothetical protein & & 40 & 0 \\
\hline 12 & NP_250447.1 & cysH & $\begin{array}{l}3^{\prime} \text {-phosphoadenosine- } 5 \text { '- } \\
\text { phosphosulfate } \\
\text { reductase }\end{array}$ & $\mathrm{L} 2 \mathrm{P}$ & 39 & 0 \\
\hline 13 & NP_248733.1 & & PA0043 & W4R & 38 & 0 \\
\hline 14 & NP_249538.1 & & diguanylate cyclase & D34G & 36 & 0 \\
\hline 15 & NP_249491.1 & & PA0800 & H78R & 36 & 0 \\
\hline 16 & NP_253724.1 & & PA5037 & & -35 & 0 \\
\hline 17 & NP_250764.1 & & PA2074 & & 33 & 0 \\
\hline 18 & NP_251980.1 & tle1 & Tle1 & N131S & 29 & 0 \\
\hline 19 & NP_253462.1 & & PA4774 & X350E & -28.5 & 0 \\
\hline 20 & NP_251980.1 & tle1 & Tle1 & I109F & 28 & 0 \\
\hline 21 & NP_249976.1 & & $\begin{array}{l}\text { PA1285 probable } \\
\text { transcriptional regulator }\end{array}$ & D3V & 27 & 0 \\
\hline 22 & NP_253781.1 & & $\begin{array}{l}\text { PA5094 probable } \\
\text { ATP-binding component } \\
\text { of ABC transporter }\end{array}$ & X277Q & -27 & 0 \\
\hline 23 & NP_250105.1 & & 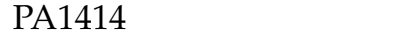 & & 27 & 0 \\
\hline 24 & NP_249292.1 & $\operatorname{agt} R$ & $\begin{array}{l}\text { two-component response } \\
\text { regulator, AgtR }\end{array}$ & $\begin{array}{l}1 \\
\text { deletion }\end{array}$ & -25 & 0 \\
\hline 25 & NP_249711.1 & & $\begin{array}{l}\text { PA1020 probable } \\
\text { acyl-CoA dehydrogenase }\end{array}$ & S8T & 25 & 0 \\
\hline 26 & NP_251148.1 & & PA2458 & & -22 & 0 \\
\hline 27 & - & & hypothetical protein & & 22 & 0 \\
\hline 28 & NP_251980.1 & tle1 & Tle1 & I231V & 21 & 0 \\
\hline 29 & NP_250876 & & PA2186 & & -21 & 0 \\
\hline 30 & NP_251089.1 & $p v d D$ & pyoverdine synthetase $\mathrm{D}$ & & -21 & 0 \\
\hline 31 & - & & hypothetical protein & & -21 & 0 \\
\hline 32 & NP_250674.1 & $\operatorname{exaC}$ & $\begin{array}{l}\text { NAD+ dependent } \\
\text { aldehyde dehydrogenase } \\
\text { ExaC }\end{array}$ & & -20.5 & 0 \\
\hline 33 & NP_251742.1 & & PA3052 & H18R & 20 & 0 \\
\hline 34 & NP_249140.1 & & PA0449 & W38R & 19 & 0 \\
\hline 35 & NP_249140.1 & & PA0449 & G39S & 19 & 0 \\
\hline 36 & - & & hypothetical protein & & 19 & 0 \\
\hline 37 & - & & hypothetical protein & & 18.5 & 0 \\
\hline 38 & - & & hypothetical protein & & 18 & 0 \\
\hline 39 & _ & & hypothetical protein & & -18 & 0 \\
\hline 40 & _ & & hypothetical protein & & 18 & 0 \\
\hline 41 & _- & & hypothetical protein & & -18 & 0 \\
\hline 42 & NP_251435.1 & & $\begin{array}{l}\text { PA2745 probable } \\
\text { hydrolase }\end{array}$ & L88G & 17 & 0 \\
\hline 43 & NP_252532 & & PA3843 & & 17 & 0 \\
\hline 44 & - & & $\begin{array}{l}\text { non-ribosomal peptide } \\
\text { synthetase (pseudogene) }\end{array}$ & & 17 & 0 \\
\hline 45 & NP_251593.1 & cobJ & $\begin{array}{l}\text { precorrin-3 methylase } \\
\text { CobJ }\end{array}$ & T4A & -16 & 0 \\
\hline
\end{tabular}




\begin{tabular}{|c|c|c|c|c|c|c|}
\hline Rank & Accession & $\begin{array}{l}\text { Gene } \\
\text { name }\end{array}$ & Protein name & Mutation & $\begin{array}{c}\text { Association } \\
\text { score }\end{array}$ & $\mathrm{p}$ value \\
\hline 46 & NP_248961.1 & & PA0270 & & -16 & 0 \\
\hline 47 & - & & hypothetical protein & & -16 & 0 \\
\hline 48 & NP_248764.1 & $p p k A$ & $\begin{array}{l}\text { serine/threonine protein } \\
\text { kinase PpkA }\end{array}$ & L295M & -15 & 0 \\
\hline 49 & NP_249454.1 & mисA & anti-sigma factor MucA & & -15 & 0 \\
\hline 50 & - & & $\begin{array}{l}\text { tryptophan repressor } \\
\text { binding protein }\end{array}$ & & 15 & 0 \\
\hline 51 & 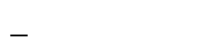 & & hypothetical protein & & 15 & 0 \\
\hline 52 & NP_249417.1 & & $\begin{array}{l}\text { PA0726 hypothetical } \\
\text { protein of bacteriophage } \\
\text { Pf1 }\end{array}$ & & -15 & 0 \\
\hline
\end{tabular}

\section{B.15. Dataset 4 random forest results}

Table 32: Genes and SNPs identified with RF from dataset 4, reduced dataset using features identified from random forest in datasets 1 and 2. Variants with importances greater than or equal to 0.002 are reported.

\begin{tabular}{|c|c|c|c|c|c|}
\hline Rank & Accession & Gene name & Protein name & Mutation & Importance \\
\hline 1 & NP_250764.1 & & PA2074 & & 0.268 \\
\hline 2 & NP_250663.1 & $p q q F$ & $\begin{array}{l}\text { pyrroloquinoline quinone } \\
\text { biosynthesis protein } \mathrm{F}\end{array}$ & $\mathrm{H} 3 \mathrm{R}$ & 0.09 \\
\hline 3 & NP_248888.1 & tonB2 & TonB2 & & 0.042 \\
\hline 4 & NP_254125.1 & & $\begin{array}{l}\text { PA5438 probable transcriptional } \\
\text { regulator }\end{array}$ & $\begin{array}{l}272 \\
\text { deletion }\end{array}$ & 0.036 \\
\hline 5 & - & & mutL (pseudogene) & & 0.027 \\
\hline 6 & NP_248708.1 & fmt & methionyl-tRNA formyltransferase & I196V & 0.022 \\
\hline 7 & NP_064723.1 & recF & RecF protein & & 0.021 \\
\hline 8 & NP_250018.1 & & PA1327 probable protease & & 0.021 \\
\hline 9 & NP_249454.1 & mисA & anti-sigma factor MucA & & 0.02 \\
\hline 10 & NP_250980.1 & $g c d$ & glucose dehydrogenase & & 0.018 \\
\hline 11 & NP_249004.1 & & $\begin{array}{l}\text { L-cysteine transporter of } \mathrm{ABC} \\
\text { system YecS }\end{array}$ & $\begin{array}{l}164 \\
\text { insertion }\end{array}$ & 0.015 \\
\hline 12 & _ & & tRNA-Ala & & 0.013 \\
\hline 13 & - & & $\begin{array}{l}\text { non-ribosomal peptide synthetase } \\
\text { (pseudogene) }\end{array}$ & & 0.012 \\
\hline 14 & NP_251134.1 & glyA2 & serine hydroxymethyltransferase & & 0.011 \\
\hline 15 & - & & $\begin{array}{l}\text { DNA-binding response regulator } \\
\text { (pseudogene) }\end{array}$ & & 0.01 \\
\hline 16 & NP_250663.1 & $p q q F$ & $\begin{array}{l}\text { pyrroloquinoline quinone } \\
\text { biosynthesis protein } \mathrm{F}\end{array}$ & $\mathrm{H} 12 \mathrm{R}$ & 0.01 \\
\hline 17 & NP_253724.1 & & PA5037 & & 0.009 \\
\hline 18 & NP_251249.1 & & PA2559 & A161S & 0.009 \\
\hline 19 & NP_252760.1 & & PA4071 & & 0.008 \\
\hline 20 & & & hypothetical protein & & 0.008 \\
\hline 21 & NP_248783.1 & tse 6 & Tse6 & $\begin{array}{l}387 \\
\text { deletion }\end{array}$ & 0.007 \\
\hline
\end{tabular}




\begin{tabular}{|c|c|c|c|c|c|}
\hline Rank & Accession & Gene name & Protein name & Mutation & Importance \\
\hline 22 & NP_250319.1 & & PA1628 probable & R48Q & 0.007 \\
\hline 23 & NP_249838.1 & & $\begin{array}{l}\text { PA1147 probable amino acid } \\
\text { permease }\end{array}$ & V396A & 0.006 \\
\hline 24 & NP_251601.1 & & $\begin{array}{l}\text { PA2911 probable TonB-dependent } \\
\text { receptor }\end{array}$ & Y684D & 0.005 \\
\hline 25 & & & $16 \mathrm{~S}$ ribosomal RNA & & 0.005 \\
\hline 26 & NP_249116.1 & $\operatorname{mex} A$ & $\begin{array}{l}\text { Resistance-Nodulation-Cell } \\
\text { Division (RND) multidrug efflux } \\
\text { membrane fusion protein MexA } \\
\text { precursor }\end{array}$ & & 0.005 \\
\hline 27 & NP_249842.1 & imm2 & pyocin S2 immunity protein & & 0.005 \\
\hline 28 & NP_250663.1 & $p q q F$ & $\begin{array}{l}\text { pyrroloquinoline quinone } \\
\text { biosynthesis protein } \mathrm{F}\end{array}$ & K92R & 0.004 \\
\hline 29 & NP_253663.1 & $\operatorname{aruH}$ & $\begin{array}{l}\text { Arginine:Pyruvate Transaminas, } \\
\text { AruH }\end{array}$ & R84Q & 0.004 \\
\hline 30 & & & hypothetical protein & & 0.004 \\
\hline 31 & NP_251114.1 & $p v d L$ & PvdL & V3938I & 0.004 \\
\hline 32 & NP_064724.1 & gyrB & DNA gyrase subunit B & S466F & 0.004 \\
\hline 33 & NP_253604.1 & nadD2 & $\begin{array}{l}\text { nicotinate mononucleotide } \\
\text { adenylyltransferase NadD2 }\end{array}$ & S7Y & 0.004 \\
\hline 34 & NP_248937.1 & & $\begin{array}{l}\text { PA0246 probable major facilitator } \\
\text { superfamily (MFS) transporter }\end{array}$ & Y43F & 0.004 \\
\hline 35 & NP_251435.1 & & PA2745 probable hydrolase & L88G & 0.003 \\
\hline 36 & NP_250415.1 & $\operatorname{psck}$ & type III export protein PscK & E137D & 0.003 \\
\hline 37 & NP_249140.1 & & PA0449 & W38R & 0.003 \\
\hline 38 & NP_249939.1 & aprF & $\begin{array}{l}\text { Alkaline protease secretion outer } \\
\text { membrane protein AprF precursor }\end{array}$ & T82A & 0.003 \\
\hline 39 & NP_253777.1 & $\operatorname{vgrG5}$ & VgrG5 & A242T & 0.003 \\
\hline 40 & - & & $\begin{array}{l}\text { 3-oxoacyl-ACP reductase } \\
\text { (pseudogene) }\end{array}$ & & 0.003 \\
\hline 41 & NP_251093.1 & $f p v G$ & FpvG & K68T & 0.003 \\
\hline 42 & NP_253820.1 & & PA5133 & & 0.003 \\
\hline 43 & NP_254209.1 & pauA6 & Glutamylpolyamine synthetase & $\mathrm{N} 276 \mathrm{H}$ & 0.003 \\
\hline 44 & -1 & & hypothetical protein & & 0.003 \\
\hline 45 & NP_252007.1 & & PA3317 & S126N & 0.003 \\
\hline 46 & NP_250809.1 & & $\begin{array}{l}\text { alcohol dehydrogenase } \\
\text { (Zn-dependent) }\end{array}$ & & 0.003 \\
\hline 47 & NP_249234.1 & & PA0543 & & 0.003 \\
\hline 48 & NP_251973.1 & & PA3283 & G26E & 0.003 \\
\hline 49 & NP_249865.1 & napA & $\begin{array}{l}\text { periplasmic nitrate reductase } \\
\text { protein NapA }\end{array}$ & & 0.003 \\
\hline 50 & NP_248783.1 & tse 6 & Tse6 & A392L & 0.003 \\
\hline 51 & NP_253522.1 & & PA4835 & A421E & 0.003 \\
\hline 52 & NP_249381.1 & $p d t A$ & $\begin{array}{l}\text { phosphate depletion regulated TPS } \\
\text { partner A, PdtA }\end{array}$ & & 0.003 \\
\hline 53 & NP_253339.1 & cUpE3 & Pilin subunit CupE3 & N19S & 0.003 \\
\hline 54 & NP_253191.1 & $o p d D$ & $\begin{array}{l}\text { Glycine-glutamate dipeptide porin } \\
\text { OpdP }\end{array}$ & E30A & 0.003 \\
\hline
\end{tabular}




\begin{tabular}{|c|c|c|c|c|c|}
\hline Rank & Accession & Gene name & Protein name & Mutation & Importance \\
\hline 55 & & & hypothetical protein & & 0.003 \\
\hline 56 & NP_252973.1 & $\operatorname{rec} D$ & $\begin{array}{l}\text { exodeoxyribonuclease } \mathrm{V} \text { alpha } \\
\text { chain }\end{array}$ & $\mathrm{A} 26 \mathrm{~V}$ & 0.003 \\
\hline 57 & NP_250470.1 & & assimilatory nitrate reductase & T549S & 0.002 \\
\hline 58 & NP_248891.1 & & PA0200 & E64D & 0.002 \\
\hline 59 & - & & hypothetical protein & & 0.002 \\
\hline 60 & NP_250687.1 & & $\begin{array}{l}\text { PA1997 probable AMP-binding } \\
\text { enzyme }\end{array}$ & $\mathrm{A} 328 \mathrm{~T}$ & 0.002 \\
\hline 61 & NP 253485.1 & & PA4797 probable transposase & & 0.002 \\
\hline 62 & NP_252918.1 & $\operatorname{pch} D$ & $\begin{array}{l}\text { pyochelin biosynthesis protein } \\
\text { PchD }\end{array}$ & & 0.002 \\
\hline 63 & NP_252773.1 & сирB3 & usher CupB3 & & 0.002 \\
\hline 64 & 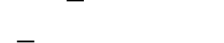 & & hemagglutinin (pseudogene) & & 0.002 \\
\hline 65 & NP_250683.1 & & $\begin{array}{l}\text { PA1993 probable major facilitator } \\
\text { superfamily (MFS) transporter }\end{array}$ & G402V & 0.002 \\
\hline 66 & NP_248921.1 & $p c a B$ & $\begin{array}{l}\text { 3-carboxy-cis,cis-muconate } \\
\text { cycloisomerase }\end{array}$ & A298T & 0.002 \\
\hline 67 & NP_252177.1 & tle5 & Tle5 & & 0.002 \\
\hline 68 & NP_253179.1 & $\operatorname{mag} D$ & MagD & M1009V & 0.002 \\
\hline 69 & NP_251838.1 & $w b p I$ & $\begin{array}{l}\text { UDP-N-acetylglucosamine } \\
\text { 2-epimerase WbpI }\end{array}$ & & 0.002 \\
\hline 70 & NP_251146 & & PA2456 & & 0.002 \\
\hline 71 & NP_248997.1 & & $\begin{array}{l}\text { PA0306 probable transcriptional } \\
\text { regulator }\end{array}$ & $\mathrm{R} 302 \mathrm{C}$ & 0.002 \\
\hline 72 & NP_251980.1 & tle1 & Tle1 & N131S & 0.002 \\
\hline 73 & NP_250871.1 & & PA2181 & & 0.002 \\
\hline 74 & NP_250674.1 & $\operatorname{exaC}$ & $\begin{array}{l}\text { NAD+ dependent aldehyde } \\
\text { dehydrogenase ExaC }\end{array}$ & & 0.002 \\
\hline 75 & _ & & hypothetical protein (pseudogene) & & 0.002 \\
\hline 76 & NP_253167.1 & $\operatorname{caf} A$ & cytoplasmic axial filament protein & T416I & 0.002 \\
\hline 77 & - & & $\begin{array}{l}\text { cystine } \mathrm{ABC} \text { transporter } \\
\text { substrate-binding protein } \\
\text { (pseudogene) }\end{array}$ & & 0.002 \\
\hline 78 & NP_252799.1 & $\operatorname{ampC}$ & beta-lactamase precursor & V239A & 0.002 \\
\hline 79 & NP_249140.1 & & PA0449 & G39S & 0.002 \\
\hline 80 & NP_253576.1 & & PA4889 probable oxidoreductase & P350S & 0.002 \\
\hline 81 & NP_249782.1 & $f g t A$ & flagellar glycosyl transferase, FgtA & & 0.002 \\
\hline 82 & & & phytanoyl-CoA dioxygenase & & 0.002 \\
\hline 83 & NP_252916.1 & pchE & dihydroaeruginoic acid synthetase & D1001E & 0.002 \\
\hline 84 & NP_250981.1 & & $\begin{array}{l}\text { PA2291 probable glucose-sensitive } \\
\text { porin }\end{array}$ & & 0.002 \\
\hline 85 & & & hypothetical protein & & 0.002 \\
\hline 86 & NP_253781.1 & & $\begin{array}{l}\text { PA5094 probable ATP-binding } \\
\text { component of ABC transporter }\end{array}$ & $\mathrm{X} 277 \mathrm{Q}$ & 0.002 \\
\hline 87 & NP_251519.1 & & PA2829 & G15A & 0.002 \\
\hline 88 & & & hypothetical protein & & 0.002 \\
\hline 89 & NP_253191.1 & opdD & $\begin{array}{l}\text { Glycine-glutamate dipeptide porin } \\
\text { OpdP }\end{array}$ & N31T & 0.002 \\
\hline
\end{tabular}




\section{B.16. Dataset 4 support vector machine results}

Table 33: Genes and SNPs identified with SVM from dataset 4, reduced dataset using features identified from random forest in datasets 1 and 2. Top 100 variants with absolute hyperplane coefficients greater than 0.03 are reported.

\begin{tabular}{|c|c|c|c|c|c|}
\hline Rank & Accession & Gene name & Protein name & Mutation & $\begin{array}{l}\text { Hyperplane } \\
\text { coefficient }\end{array}$ \\
\hline 1 & NP_250663.1 & $p q q F$ & $\begin{array}{l}\text { pyrroloquinoline quinone } \\
\text { biosynthesis protein F }\end{array}$ & H12R & 0.9060 \\
\hline 2 & NP_250663.1 & $p q q F$ & $\begin{array}{l}\text { pyrroloquinoline quinone } \\
\text { biosynthesis protein } \mathrm{F}\end{array}$ & $\mathrm{H} 3 \mathrm{R}$ & -0.6211 \\
\hline 3 & NP_254125.1 & & $\begin{array}{l}\text { PA5438 probable transcriptional } \\
\text { regulator }\end{array}$ & $\begin{array}{l}272 \\
\text { deletion }\end{array}$ & -0.5358 \\
\hline 4 & NP_251831.1 & $w b p M$ & $\begin{array}{l}\text { nucleotide sugar } \\
\text { epimerase/dehydratase WbpM }\end{array}$ & V113P & -0.5228 \\
\hline 5 & NP_248708.1 & fmt & methionyl-tRNA formyltransferase & I196V & 0.5130 \\
\hline 6 & NP_250663.1 & $p q q F$ & $\begin{array}{l}\text { pyrroloquinoline quinone } \\
\text { biosynthesis protein } \mathrm{F}\end{array}$ & K92R & -0.4968 \\
\hline 7 & NP_249976.1 & & $\begin{array}{l}\text { PA1285 probable transcriptional } \\
\text { regulator }\end{array}$ & D3V & 0.4829 \\
\hline 8 & NP_253053.1 & $i c i A$ & $\begin{array}{l}\text { inhibitor of chromosome initiation } \\
\text { IciA }\end{array}$ & M1L & 0.4712 \\
\hline 9 & NP_250447.1 & cys $H$ & $\begin{array}{l}\text { 3'-phosphoadenosine- } 5^{\prime}- \\
\text { phosphosulfate } \\
\text { reductase }\end{array}$ & L2P & -0.4707 \\
\hline 10 & NP_248733.1 & & PA0043 & W4R & -0.4622 \\
\hline 11 & NP_249939.1 & aprF & $\begin{array}{l}\text { Alkaline protease secretion outer } \\
\text { membrane protein AprF precursor }\end{array}$ & T82A & 0.4617 \\
\hline 12 & NP_250442.1 & & PA1751 1 & $\begin{array}{l}1 \\
\text { insertion }\end{array}$ & 0.4608 \\
\hline 13 & NP_251249.1 & & PA2559 & A161S & 0.4548 \\
\hline 14 & NP_250319.1 & & $\begin{array}{l}\text { PA1628 probable } \\
\text { 3-hydroxyacyl-CoA dehydrogenase }\end{array}$ & R48Q & 0.4525 \\
\hline 15 & NP_249004.1 & & $\begin{array}{l}\text { L-cysteine transporter of } \mathrm{ABC} \\
\text { system YecS }\end{array}$ & $\begin{array}{l}164 \\
\text { insertion }\end{array}$ & -0.4520 \\
\hline 16 & NP_251435.1 & & PA2745 probable hydrolase & L88G & 0.4464 \\
\hline 17 & NP_250108.1 & & PA1417 probable decarboxylase & T262A & 0.4444 \\
\hline 18 & NP_249286.1 & $\operatorname{lptD}$ & LPS-assembly protein LptD & E812K & 0.4416 \\
\hline 19 & NP_251993.1 & & $\begin{array}{l}\text { PA3303 probable major facilitator } \\
\text { superfamily (MFS) transporter }\end{array}$ & $\mathrm{H} 262 \mathrm{R}$ & -0.4302 \\
\hline 20 & NP_250663.1 & $p q q F$ & $\begin{array}{l}\text { pyrroloquinoline quinone } \\
\text { biosynthesis protein } \mathrm{F}\end{array}$ & C240G & 0.4301 \\
\hline 21 & NP_250310.1 & & $\begin{array}{l}\text { PA1619 probable transcriptional } \\
\text { regulator }\end{array}$ & $\mathrm{I} 14 \mathrm{~V}$ & 0.4205 \\
\hline 22 & NP_251980.1 & tle1 & Tle1 & I109F & -0.4158 \\
\hline 23 & NP_251358 & & PA2668 & $\begin{array}{l}5 \\
\text { insertion }\end{array}$ & -0.4119 \\
\hline 24 & NP_251232.1 & & PA2542 & $\begin{array}{l}1130 \\
\text { insertion }\end{array}$ & -0.4111 \\
\hline
\end{tabular}


B. RESULTS

\begin{tabular}{|c|c|c|c|c|c|}
\hline Rank & Accession & Gene name & Protein name & Mutation & $\begin{array}{l}\text { Hyperplane } \\
\text { coefficient }\end{array}$ \\
\hline 25 & NP_251315.1 & & PA2625 & V84I & -0.4084 \\
\hline 26 & NP_249533.1 & & $\begin{array}{l}\text { PA0842 probable glycosyl } \\
\text { transferase }\end{array}$ & K251R & -0.4048 \\
\hline 27 & NP_252886.1 & bfiS & BfiS & E431A & 0.4030 \\
\hline 28 & NP_249622.1 & pirA & ferric enterobactin receptor PirA & A370T & -0.4014 \\
\hline 29 & NP_249286.1 & lptD & LPS-assembly protein LptD & D593N & 0.3995 \\
\hline 30 & NP_252466.1 & $x \operatorname{se} A$ & $\begin{array}{l}\text { exodeoxyribonuclease VII large } \\
\text { subunit }\end{array}$ & L325Q & 0.3975 \\
\hline 31 & NP_253191.1 & opdD & $\begin{array}{l}\text { Glycine-glutamate dipeptide porin } \\
\text { OpdP }\end{array}$ & E30A & 0.3882 \\
\hline 32 & NP_248937.1 & & $\begin{array}{l}\text { PA0246 probable major facilitator } \\
\text { superfamily (MFS) transporter }\end{array}$ & Y43F & -0.3803 \\
\hline 33 & NP_251716.1 & & PA3026 & E464Q & 0.3742 \\
\hline 34 & NP_251210.1 & $c z c A$ & $\begin{array}{l}\text { Resistance-Nodulation-Cell } \\
\text { Division (RND) divalent metal } \\
\text { cation efflux transporter CzcA }\end{array}$ & R595K & 0.3720 \\
\hline 35 & NP_253957.1 & & PA5270 & S5A & 0.3671 \\
\hline 36 & NP_248783.1 & tse 6 & Tse6 & $\begin{array}{l}387 \\
\text { deletion }\end{array}$ & -0.3646 \\
\hline 37 & NP_248891.1 & & PA0200 & E64D & 0.3617 \\
\hline 38 & NP_253549.1 & & $\begin{array}{l}\text { PA4862 probable ATP-binding } \\
\text { component of ABC transporter }\end{array}$ & A174T & -0.3613 \\
\hline 39 & NP_251601.1 & & $\begin{array}{l}\text { PA2911 probable TonB-dependent } \\
\text { receptor }\end{array}$ & Y684D & -0.3572 \\
\hline 40 & NP_248783.1 & tse6 & Tse6 & A392L & -0.3530 \\
\hline 41 & NP_253939.1 & & $\begin{array}{l}\text { PA5252 probable ATP-binding } \\
\text { component of ABC transporter }\end{array}$ & T225I & -0.3479 \\
\hline 42 & NP_253462.1 & & PA4774 & X350E & 0.3470 \\
\hline 43 & NP_252350.1 & & $\begin{array}{l}\text { PA3660 probable } \\
\text { sodium/hydrogen antiporter }\end{array}$ & A14T & -0.3449 \\
\hline 44 & NP_248969.1 & & PA0278 & V111A & 0.3422 \\
\hline 45 & NP_253191.1 & opdD & $\begin{array}{l}\text { Glycine-glutamate dipeptide porin } \\
\text { OpdP }\end{array}$ & $\mathrm{N} 31 \mathrm{~T}$ & 0.3377 \\
\hline 46 & NP_252017.1 & & $\begin{array}{l}\text { PA3327 probable non-ribosomal } \\
\text { peptide synthetase }\end{array}$ & E402K & -0.3365 \\
\hline 47 & NP_251604.1 & & $\begin{array}{l}\text { PA2914 probable permease of ABC } \\
\text { transporter }\end{array}$ & $\mathrm{I} 255 \mathrm{~V}$ & -0.3307 \\
\hline 48 & NP_251115.1 & $p v d G$ & PvdG & D178E & -0.3277 \\
\hline 49 & NP_251358 & & PA2668 & R4L & 0.3271 \\
\hline 50 & NP_249925.1 & & PA1234 & N39D & -0.3233 \\
\hline 51 & NP_254006.1 & $\mathrm{radC}$ & DNA repair protein RadC & D212G & -0.3174 \\
\hline 52 & NP_250687.1 & & $\begin{array}{l}\text { PA1997 probable AMP-binding } \\
\text { enzyme }\end{array}$ & A328T & 0.3173 \\
\hline 53 & NP_251593.1 & cobJ & precorrin-3 methylase CobJ & T4A & 0.3170 \\
\hline 54 & NP_248764.1 & $p p k A$ & $\begin{array}{l}\text { serine/threonine protein kinase } \\
\text { PpkA }\end{array}$ & L295M & -0.3158 \\
\hline 55 & NP_253663.1 & aruH & $\begin{array}{l}\text { Arginine:Pyruvate Transaminas, } \\
\text { AruH }\end{array}$ & R84Q & -0.3158 \\
\hline
\end{tabular}


B. RESULTS

\begin{tabular}{|c|c|c|c|c|c|}
\hline Rank & Accession & Gene name & Protein name & Mutation & $\begin{array}{l}\text { Hyperplane } \\
\text { coefficient }\end{array}$ \\
\hline 56 & NP_252007.1 & & PA3317 & S126N & 0.3148 \\
\hline 57 & NP_249290.1 & & PA0599 & A20T & 0.3140 \\
\hline 58 & NP_250415.1 & $\operatorname{psck}$ & type III export protein PscK & E137D & -0.3120 \\
\hline 59 & NP_253413.1 & $\operatorname{cbrA}$ & two-component sensor CbrA & T16A & -0.3100 \\
\hline 60 & NP_250612.1 & & $\begin{array}{l}\text { PA1922 probable TonB-dependent } \\
\text { receptor }\end{array}$ & V419A & 0.3100 \\
\hline 61 & NP_250774.1 & & $\begin{array}{l}\text { PA2084 probable asparagine } \\
\text { synthetase }\end{array}$ & V118A & 0.3084 \\
\hline 62 & NP 251003 & & PA2313 & $\mathrm{H} 205 \mathrm{O}$ & 0.3076 \\
\hline 63 & NP_250806.1 & & PA2116 & T148A & 0.3001 \\
\hline 64 & NP_250939.1 & $b k d B$ & $\begin{array}{l}\text { branched-chain alpha-keto acid } \\
\text { dehydrogenase (lipoamide } \\
\text { component) }\end{array}$ & S321G & 0.2972 \\
\hline 65 & NP_251749.1 & pelF & PelF & N229S & 0.2967 \\
\hline 66 & NP_251519.1 & & PA2829 & G15A & 0.2958 \\
\hline 67 & NP_250692.1 & & PA2002 & V457G & 0.2916 \\
\hline 68 & NP_251005.1 & & PA2315 & V284I & -0.2892 \\
\hline 69 & NP_249124.1 & & PA0433 & H116Q & -0.2890 \\
\hline 70 & NP_249633.1 & & $\begin{array}{l}\text { PA0942 probable transcriptional } \\
\text { regulator }\end{array}$ & D172A & 0.2835 \\
\hline 71 & NP_254011.1 & $\operatorname{sph} R$ & $\begin{array}{l}\text { Sphingosine-responsive Regulator, } \\
\text { SphR }\end{array}$ & D347E & -0.2818 \\
\hline 72 & NP_251124.1 & & PA2434 & A145T & -0.2816 \\
\hline 73 & NP_251005.1 & & PA2315 & L306V & -0.2811 \\
\hline 74 & NP_250470.1 & & assimilatory nitrate reductase & T549S & 0.2801 \\
\hline 75 & NP_251358 & & PA2668 & R4I & 0.2779 \\
\hline 76 & NP_251157.1 & foxR & Anti-sigma factor FoxR & A28G & 0.2770 \\
\hline 77 & NP_252126.1 & & PA3436 & H92R & -0.2762 \\
\hline 78 & NP_253777.1 & vgrG5 & VgrG5 & A242T & 0.2760 \\
\hline 79 & NP_253179.1 & $\operatorname{mag} D$ & MagD & M1009V & 0.2736 \\
\hline 80 & NP_251602.1 & & $\begin{array}{l}\text { PA2912 probable ATP-binding } \\
\text { component of ABC transporter }\end{array}$ & S185G & 0.2735 \\
\hline 81 & NP_249292.1 & $\operatorname{agt} R$ & $\begin{array}{l}\text { two-component response regulator, } \\
\text { AgtR }\end{array}$ & $\begin{array}{l}1 \\
\text { deletion }\end{array}$ & -0.2729 \\
\hline 82 & NP_249158.1 & & PA0467 & G140A & 0.2711 \\
\hline 83 & NP_251114.1 & $p v d L$ & PvdL & V3938I & -0.2711 \\
\hline 84 & YP_008719755.1 & $1 \operatorname{srf} A$ & SrfA & F87R & -0.2688 \\
\hline 85 & NP_253669 & & $\begin{array}{l}\text { PA4982 probable two-component } \\
\text { sensor }\end{array}$ & N904S & -0.2685 \\
\hline 86 & NP_250356.1 & fha2 & Fha2 & G277S & -0.2681 \\
\hline 87 & NP_250705.1 & liuA & $\begin{array}{l}\text { putative isovaleryl-CoA } \\
\text { dehydrogenase }\end{array}$ & A179P & -0.2678 \\
\hline 88 & NP_249019.1 & $a a a A$ & $\begin{array}{l}\text { arginine-specific autotransporter of } \\
\text { Pseudomonas aeruginosa, AaaA }\end{array}$ & H516R & -0.2677 \\
\hline 89 & NP_250023.1 & & 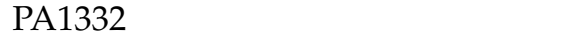 & C48R & -0.2667 \\
\hline 90 & NP_250630.1 & & PA1940 & T284A & 0.2664 \\
\hline 91 & NP_252454.1 & & PA3765 & S62R & 0.2649 \\
\hline 92 & NP_252030.1 & & PA3340 & S181N & 0.2647 \\
\hline
\end{tabular}




\begin{tabular}{|c|c|c|c|c|c|}
\hline Rank & Accession & Gene name & Protein name & Mutation & $\begin{array}{l}\text { Hyperplane } \\
\text { coefficient }\end{array}$ \\
\hline 93 & NP_253231.1 & $\operatorname{lep} A$ & $\begin{array}{l}\text { Pseudomonas aeruginosa-derived } \\
\text { large extracellular protease, LepA }\end{array}$ & S550N & -0.2647 \\
\hline 94 & NP_251970.1 & oprO & $\begin{array}{l}\text { Pyrophosphate-specific outer } \\
\text { membrane porin OprO precursor }\end{array}$ & F86Y & 0.2638 \\
\hline 95 & NP_251813.1 & & PA3123 & S37T & -0.2634 \\
\hline 96 & NP_251980.1 & tle1 & Tle1 & N131S & 0.2618 \\
\hline 97 & NP_249399.1 & & $\begin{array}{l}\text { PA0708 probable transcriptional } \\
\text { regulator }\end{array}$ & G181S & 0.2617 \\
\hline 98 & NP_249104.1 & $\operatorname{chp} A$ & $\begin{array}{l}\text { component of chemotactic signal } \\
\text { transduction system }\end{array}$ & V594L & 0.2615 \\
\hline 99 & NP_249104.1 & $\operatorname{chp} A$ & $\begin{array}{l}\text { component of chemotactic signal } \\
\text { transduction system }\end{array}$ & E825A & -0.2612 \\
\hline 100 & NP_252913.1 & & $\begin{array}{l}\text { PA4223 probable ATP-binding } \\
\text { component of ABC transporter }\end{array}$ & S484R & 0.2602 \\
\hline
\end{tabular}

\section{Additional Test Results}

C.1. Results from random forest after condensing dataset 1 using $95 \%$ similarity profiles

Table 34: Importance of genes in random forest models before and after condensing the dataset using a $95 \%$ similarity threshold. If genes shared $95 \%$ of the same presence/absence profile with another gene(s), the genes were condensed into a single variable. The majority of genes condensed were either core genes or genes found in PA during a longitudinal study of one patient.

\begin{tabular}{llll}
\hline Accession & Protein name & Importance & $\begin{array}{l}\text { Original } \\
\text { Importance }\end{array}$ \\
\hline NP_250764.1 & PA2074 & 0.28 & 0.237 \\
NP_249454.1 & MucA & 0.116 & 0.123 \\
NP_253724.1 & PA5037 & 0.061 & 0.041 \\
NP_248888.1 & TonB2 & 0.032 & 0.084 \\
NP_251800.1 & PA3110 & 0.022 & 0 \\
NP_249415.1 & probable coat protein A of & 0.014 & 0.005 \\
& bacteriophage Pf1 & & \\
NP_251184.1 & MexF & 0.011 & 0.011 \\
NP_252532 & PA3843 & 0.011 & 0.022 \\
NP_064723.1 & RecF & 0.01 & 0.029 \\
& hypothetical protein & 0.009 & 0 \\
& (pseudogene) & & \\
& MutL (pseudogene) & 0.008 & 0.009 \\
& non-ribosomal peptide & 0.008 & 0.015 \\
& synthetase (pseudogene) & & \\
NP_249842.1 & pyocin S2 immunity protein & 0.007 & 0.004 \\
& DNA-binding response & 0.007 & 0.01 \\
& regulator (pseudogene) & & \\
\hline
\end{tabular}




\begin{tabular}{|c|c|c|c|}
\hline Accession & Protein name & Importance & $\begin{array}{l}\text { Original } \\
\text { Importance }\end{array}$ \\
\hline NP_249929.1 & $\begin{array}{l}\text { probable outer membrane } \\
\text { component of multidrug efflux } \\
\text { pump }\end{array}$ & 0.006 & 0.009 \\
\hline \multirow[t]{5}{*}{ NP_250710.1 } & MexZ & 0.005 & 0 \\
\hline & ffs & 0.005 & 0 \\
\hline & $\begin{array}{l}\text { 3-oxoacyl-ACP reductase } \\
\text { (pseudogene) }\end{array}$ & 0.005 & 0.002 \\
\hline & hypothetical protein & 0.005 & 0 \\
\hline & nan & 0.005 & 0 \\
\hline NP_249381.1 & $\begin{array}{l}\text { phosphate depletion regulated } \\
\text { TPS partner A, PdtA }\end{array}$ & 0.004 & 0.008 \\
\hline \multirow[t]{2}{*}{ NP_250809.1 } & $\begin{array}{l}\text { alcohol dehydrogenase } \\
\text { (Zn-dependent) }\end{array}$ & 0.003 & 0.001 \\
\hline & nan & 0.003 & 0 \\
\hline \multirow[t]{2}{*}{ NP_251015.1 } & PA2325 & 0.003 & 0 \\
\hline & $\begin{array}{l}\text { efflux transporter periplasmic } \\
\text { adaptor subunit (pseudogene) }\end{array}$ & 0.003 & 0.002 \\
\hline NP_253820.1 & PA5133 & 0.003 & 0.008 \\
\hline \multirow{3}{*}{ NP_249673.1 } & PA0982 & 0.003 & 0 \\
\hline & nan & 0.003 & 0.004 \\
\hline & $\begin{array}{l}\text { ABC transporter ATP-binding } \\
\text { protein (pseudogene) }\end{array}$ & 0.003 & 0 \\
\hline NP_252709.1 & $\begin{array}{l}\text { UDP-N-acetylmuramate:L- } \\
\text { alanyl-gamma-D-glutamyl- } \\
\text { meso-diaminopimelate } \\
\text { ligase }\end{array}$ & 0.002 & 0.002 \\
\hline \multirow[t]{3}{*}{ NP_248790.1 } & PA0100 & 0.002 & 0 \\
\hline & $\begin{array}{l}\text { cystine } \mathrm{ABC} \text { transporter } \\
\text { substrate-binding protein } \\
\text { (pseudogene) }\end{array}$ & 0.002 & 0.003 \\
\hline & $\begin{array}{l}\text { hypothetical protein } \\
\text { (pseudogene) }\end{array}$ & 0.002 & 0.001 \\
\hline NP_249865.1 & $\begin{array}{l}\text { periplasmic nitrate reductase } \\
\text { protein NapA }\end{array}$ & 0.002 & 0.003 \\
\hline \multirow[t]{6}{*}{ NP_252177.1 } & Tle5 & 0.002 & 0.005 \\
\hline & transcriptional regulator & 0.002 & 0.003 \\
\hline & glyA (pseudogene) & 0.002 & 0.001 \\
\hline & hypothetical protein & 0.002 & 0 \\
\hline & [] & 0.002 & 0 \\
\hline & $\begin{array}{l}\text { TetR family transcriptional } \\
\text { regulator (pseudogene) }\end{array}$ & 0.002 & 0.005 \\
\hline \multirow[t]{2}{*}{ NP_250118 } & PA1427 & 0.002 & 0.006 \\
\hline & $\begin{array}{l}\text { polyhydroxybutyrate } \\
\text { depolymerase (pseudogene) }\end{array}$ & 0.001 & 0 \\
\hline \multirow[t]{2}{*}{ NP_249677.1 } & PA0986 & 0.001 & 0 \\
\hline & hypothetical protein & 0.001 & 0 \\
\hline \multirow[t]{2}{*}{ NP_252057.1 } & PA3367 & 0.001 & 0 \\
\hline & hypothetical protein & 0.001 & 0 \\
\hline
\end{tabular}




\begin{tabular}{|c|c|c|c|}
\hline Accession & Protein name & Importance & $\begin{array}{l}\text { Original } \\
\text { Importance }\end{array}$ \\
\hline \multirow[t]{4}{*}{ NP_251876.1 } & $\begin{array}{l}\text { Glucose/carbohydrate outer } \\
\text { membrane porin OprB } \\
\text { precursor }\end{array}$ & 0.001 & 0 \\
\hline & emrB (pseudogene) & 0.001 & 0 \\
\hline & [] & 0.001 & 0 \\
\hline & hypothetical protein & 0.001 & 0.001 \\
\hline \multirow[t]{5}{*}{ NP_251028.1 } & $\begin{array}{l}\text { probable binding protein } \\
\text { component of } \mathrm{ABC}\end{array}$ & 0.001 & 0 \\
\hline & $\begin{array}{l}\text { maltose/mannitol transporter } \\
\text { subtype I-F CRISPR-associated } \\
\text { endonuclease Cas1 }\end{array}$ & 0.001 & 0 \\
\hline & peptidase & 0.001 & 0.003 \\
\hline & nan & 0.001 & 0 \\
\hline & hypothetical protein & 0.001 & 0 \\
\hline \multirow[t]{7}{*}{ NP_251838.1 } & UDP-N-acetylglucosamine & 0.001 & 0.002 \\
\hline & hypothetical protein & 0.001 & 0 \\
\hline & nan & 0.001 & 0.004 \\
\hline & hydrolase (pseudogene) & 0.001 & 0 \\
\hline & O-antigen biosynthesis protein & 0.001 & 0 \\
\hline & [] & 0.001 & 0 \\
\hline & $\begin{array}{l}\text { UDP-N-acetylmuramate:L- } \\
\text { alanyl-gamma-D-glutamyl- } \\
\text { meso-diaminopimelate ligase } \\
\text { (pseudogene) }\end{array}$ & 0.001 & 0.002 \\
\hline \multirow[t]{6}{*}{ NP_250420.1 } & PA1729 & 0.001 & 0 \\
\hline & nan & 0.001 & 0 \\
\hline & hypothetical protein & 0.001 & 0 \\
\hline & hypothetical protein & 0.001 & 0 \\
\hline & hypothetical protein & 0.001 & 0 \\
\hline & hypothetical protein & 0.001 & 0 \\
\hline \multirow[t]{2}{*}{ NP_250162.1 } & 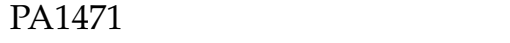 & 0.001 & 0 \\
\hline & $\begin{array}{l}\text { hypothetical protein similar to } \\
\text { coat protein A of bacteriophage } \\
\text { Pf1 }\end{array}$ & 0.001 & 0 \\
\hline \multirow[t]{2}{*}{ NP_250203.1 } & secreted protein Hcp & 0.001 & 0 \\
\hline & paraquat-inducible protein $\mathrm{A}$ & 0.001 & 0 \\
\hline NP_251134.1 & $\begin{array}{l}\text { serine } \\
\text { hydroxymethyltransferase }\end{array}$ & 0.001 & 0.005 \\
\hline \multirow[t]{2}{*}{ NP_250074.1 } & PA1383 & 0.001 & 0.005 \\
\hline & [] & 0.001 & 0 \\
\hline NP_251508.1 & $\begin{array}{l}\text { aminoglycoside response } \\
\text { regulator }\end{array}$ & 0.001 & 0 \\
\hline NP_250059.1 & PA1368 & 0.001 & 0.002 \\
\hline NP_251031.1 & $\begin{array}{l}\text { probable ATP-binding } \\
\text { component of ABC } \\
\text { maltose/mannitol transporter } \\
\text { beta-lactamase }\end{array}$ & 0.001 & 0 \\
\hline
\end{tabular}




\begin{tabular}{|c|c|c|c|}
\hline Accession & Protein name & Importance & $\begin{array}{l}\text { Original } \\
\text { Importance }\end{array}$ \\
\hline \multirow[t]{10}{*}{ NP_250018.1 } & probable protease & 0.001 & 0.004 \\
\hline & [] & 0.001 & 0 \\
\hline & [] & 0.001 & 0 \\
\hline & $\begin{array}{l}\text { pilus assembly protein FimV } \\
\text { (pseudogene) }\end{array}$ & 0.001 & 0 \\
\hline & nan & 0.001 & 0 \\
\hline & nan & 0.001 & 0 \\
\hline & nan & 0.001 & 0 \\
\hline & hypothetical protein & 0.001 & 0 \\
\hline & $\begin{array}{l}\text { chemotaxis protein } \\
\text { (pseudogene) }\end{array}$ & 0.001 & 0 \\
\hline & hypothetical protein & 0.001 & 0.001 \\
\hline \multirow[t]{3}{*}{ NP_251618.1 } & PA2928 & 0.001 & 0 \\
\hline & hypothetical protein & 0.001 & 0 \\
\hline & hypothetical protein & 0.001 & 0 \\
\hline \multicolumn{2}{|c|}{ YP_008719781.1PA4638 } & 0.001 & 0 \\
\hline & nan & 0.001 & 0 \\
\hline & hypothetical protein & 0.001 & 0 \\
\hline NP_253389.1 & PA4701 & 0.001 & 0 \\
\hline \multirow[t]{6}{*}{ NP_253378.1 } & PA4689 & 0.001 & 0 \\
\hline & hypothetical protein & 0.001 & 0 \\
\hline & nan & 0.001 & 0.001 \\
\hline & nan & 0.001 & 0 \\
\hline & acetyl-CoA acetyltransferase & 0.001 & 0 \\
\hline & [] & 0.001 & 0.001 \\
\hline \multirow[t]{2}{*}{ NP_250908.1 } & PA2218 & 0.001 & 0 \\
\hline & hemagglutinin (pseudogene) & 0.001 & 0 \\
\hline \multirow[t]{4}{*}{ NP_248948.1 } & PA0257 & 0.001 & 0.006 \\
\hline & hypothetical protein & 0.001 & 0 \\
\hline & hypothetical protein & 0.001 & 0 \\
\hline & hypothetical protein & 0.001 & 0 \\
\hline \multirow[t]{10}{*}{ NP_249374.1 } & $\mathrm{HxcY}$ & 0.001 & 0.004 \\
\hline & nan & 0.001 & 0 \\
\hline & hypothetical protein & 0.001 & 0 \\
\hline & $\begin{array}{l}\text { cobyrinic acid a,c-diamide } \\
\text { synthase }\end{array}$ & 0.001 & 0 \\
\hline & 5S ribosomal RNA & 0.001 & 0 \\
\hline & hypothetical protein & 0.001 & 0.003 \\
\hline & hypothetical protein & 0.001 & 0 \\
\hline & hydrolase & 0.001 & 0 \\
\hline & nan & 0.001 & 0.002 \\
\hline & hypothetical protein & 0.001 & 0 \\
\hline \multirow[t]{2}{*}{ NP_249252.1 } & hypothetical protein & 0.001 & 0 \\
\hline & lipoprotein & 0.001 & 0.001 \\
\hline NP_250876 & PA2186 & 0.001 & 0.002 \\
\hline \multirow[t]{2}{*}{ NP_253664.1 } & $\begin{array}{l}\text { 2-ketoarginine decarboxylase, } \\
\text { AruI }\end{array}$ & 0.001 & 0.002 \\
\hline & hypothetical protein & 0.001 & 0.002 \\
\hline
\end{tabular}




\begin{tabular}{|c|c|c|c|}
\hline Accession & Protein name & Importance & $\begin{array}{l}\text { Original } \\
\text { Importance }\end{array}$ \\
\hline NP 249316.1 & PA0625 & 0.001 & 0 \\
\hline \multirow[t]{3}{*}{ NP_249188.1 } & PA0497 & 0.001 & 0 \\
\hline & $\begin{array}{l}\text { hypothetical protein } \\
\text { (pseudogene) }\end{array}$ & 0.001 & 0 \\
\hline & $\begin{array}{l}\text { sigma-54-dependent Fis family } \\
\text { transcriptional regulator } \\
\text { (pseudogene) }\end{array}$ & 0.001 & 0 \\
\hline NP_248987.1 & Glutamylpolyamine synthetase & 0.001 & 0 \\
\hline \multirow[t]{2}{*}{ NP_252906.1 } & $\begin{array}{l}\text { probable pyridoxamine } \\
5^{\prime} \text {-phosphate oxidase }\end{array}$ & 0.001 & 0 \\
\hline & nan & 0.001 & 0 \\
\hline \multirow[t]{2}{*}{ NP_252903.1 } & $\begin{array}{l}\text { phenazine biosynthesis protein } \\
\text { PhzE }\end{array}$ & 0.001 & 0 \\
\hline & $\begin{array}{l}\text { ABC transporter } \\
\text { substrate-binding protein }\end{array}$ & 0.001 & 0 \\
\hline NP_252711.1 & $\begin{array}{l}\text { hydrazone dehydrogenase, } \\
\text { HdhA }\end{array}$ & 0.001 & 0 \\
\hline \multirow[t]{4}{*}{ NP_249603.1 } & PA0912 & 0.001 & 0 \\
\hline & DNA-binding protein & 0.001 & 0.003 \\
\hline & [] & 0.001 & 0 \\
\hline & hypothetical protein & 0.001 & 0 \\
\hline \multirow[t]{4}{*}{ NP_251011.1 } & gluconokinase & 0.001 & 0 \\
\hline & hypothetical protein & 0.001 & 0 \\
\hline & cysteine synthase (pseudogene) & 0.001 & 0 \\
\hline & hypothetical protein & 0.001 & 0 \\
\hline \multirow[t]{7}{*}{ NP_250922.1 } & PslB & 0.001 & 0.001 \\
\hline & $\begin{array}{l}\text { carboxylate-amine ligase } \\
\text { (pseudogene) }\end{array}$ & 0.001 & 0.001 \\
\hline & tRNA-Ile & 0.001 & 0 \\
\hline & nan & 0.001 & 0 \\
\hline & hypothetical protein & 0.001 & 0 \\
\hline & acetyltransferase & 0.001 & 0 \\
\hline & $\begin{array}{l}\text { ABC transporter ATP-binding } \\
\text { protein (pseudogene) }\end{array}$ & 0.001 & 0 \\
\hline
\end{tabular}

